\title{
Coupling of a Single Nitrogen-Vacancy Center in Diamond to a Fiber-based Microcavity
}

\author{
Dissertation \\ zur Erlangung des Grades \\ des Doktors der Naturwissenschaften \\ der Naturwissenschaftlich-Technischen Fakultät II \\ - Physik und Mechatronik - \\ der Universität des Saarlandes
}

von

Roland Christoph Albrecht

Saarbrücken

2014 
Tag des Kolloquiums:

Dekan:

Mitglieder des Prüfungsausschusses:
07.05 .2014

Univ.-Prof. Dr. rer. nat. Christian Wagner

Univ.-Prof. Dr. rer. nat. Jürgen Eschner Univ.-Prof. Dr. rer. nat. Christoph Becher Dr. Jason Smith

Dr. rer. nat. Christian Zeitz 
Is this a dagger which I see before me, The handle toward my hand? Come let me clutch thee.

I have thee not, and yet I see thee still. Art thou not fatal vision, sensible To feeling as to sight? or art thou but A dagger of the mind, a false creation, Proceeding from the heat-opressed brain?

-W. Shakespeare - Macbeth, Act II, Scene I 


\begin{abstract}
This work describes the coupling of a single nitrogen-vacancy (NV) center in diamond to a fiber-based Fabry-Perot cavity. To realize the cavities, concave imprints on fiber facets are produced by laser machining or focused ion beam milling and subsequently coated with a highly reflective mirror-stack. Nanodiamonds containing single NV centers are incorporated into cavities, that consist either of one plane mirror and one fiber mirror or of two fiber mirrors. Characterizing both the emission into free-space and the cavity-coupled emission of the very same NV center allows to demonstrate a cavityenhanced emission: 2-4\% of the total emission of the NV center is directed into the cavity mode with a linewidth of approximately $10 \mathrm{GHz}$. We thereby have realized a tunable narrow-band single photon source. Starting from a master equation approach a rate equation model is deduced that provides insights into the underlying physical processes: the emitter-cavity coupling leads to phonon induced off-resonant channeling of the emission into the narrow cavity mode. The measurements of the cavity emission rate at different wavelengths are well reproduced by this model. The results of this work are promising for future applications in quantum information science such as realization of a source of indistinguishable photons, cavity enhanced spin read-out of the NV center and realization of a spin-photon interface for use in quantum networks.
\end{abstract}

\title{
Zusammenfassung
}

Diese Arbeit beschreibt die Ankopplung eines einzelnen Stickstoff-Fehlstellen (NV) Zentrums in Diamant an einen faserbasierten Fabry-Perot Mikro-Resonator. Für deren Realisierung werden konkave Strukturen auf Faser-Facetten durch Lasermaterialbearbeitung oder fokussierte Ionenstrahl-Abtragung hergestellt und anschließend hochreflektiv beschichtet. Nanodiamanten, welche einzelne NVs enthalten, werden in den Resonator eingebracht, welcher entweder aus einem Planspiegel und einem Faserspiegel oder zwei Faserspiegeln besteht. Durch Charakterisierung der Abstrahlung in den freien Raum und der Resonator-gekoppelten Emission des gleichen NV Zentrums kann eine überhöhte Abstrahlung in den Resonator nachgewiesen werden: 2-4\% der gesamten Emission des NV Zentrums erfolgt in die schmale Resonanz mit einer Breite von circa $10 \mathrm{GHz}$. Ausgehend von einem Mastergleichungsansatz wird ein Raten-Modell entwickelt, welches ein physikalisches Bild der ablaufenden Prozesse vermittelt. Es findet eine durch Phononen induzierte, nicht-resonante Kanalisierung der Emission in die Resonatormode statt. Die Messungen der Resonatoremission als Funktion der Wellenlänge werden sehr genau durch das Modell reproduziert. Die Ergebnisse dieser Arbeit sind vielversprechend im Hinblick auf Anwendungen in der Quanteninformationsverarbeitung wie z.B. die Realisierung von Quellen einzelner ununterscheidbarer Photonen oder die Realisierung einer Spin-Photon Schnittstelle zur Anwendung in Quantennetzwerken. 


\section{Contents}

1 Introduction 1

1.1 The nitrogen-vacancy center in diamond . . . . . . . . . . . . 1

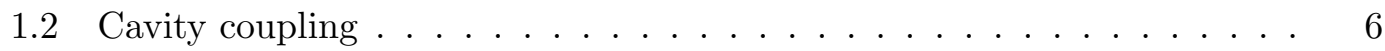

1.3 Scope of this thesis . . . . . . . . . . . . . . . . 10

2 Theoretical background $\quad 11$

2.1 Nitrogen-vacancy center in diamond . . . . . . . . . . . . . . . . 11

2.1 .1 Overview ............................. 11

2.1 .2 Electronic structure . . . . . . . . . . . . . . . . . 13

2.1.3 Vibronic structure and optical properties . . . . . . . . . . 17

2.2 Fabry-Perot cavities . . . . . . . . . . . . . . . . . . 24

2.2 .1 Gaussian beam . . . . . . . . . . . . . . . . 27

2.2 .2 Resonators with spherical mirrors . . . . . . . . . . . . 28

2.3 Cavity quantum electrodynamics . . . . . . . . . . . . . . 30

2.3.1 Jaynes-Cummings Hamiltonian . . . . . . . . . . . . . . . . 30

2.3.2 Master equation . . . . . . . . . . . . . . . . . . 32

2.3.3 Dissipation - coupling regimes . . . . . . . . . . . . . . 32

2.4 Single nitrogen-vacancy center in a microcavity . . . . . . . . . . . . 35

2.4.1 Conventions . . . . . . . . . . . . . . . . . . 35

2.4 .2 Model . . . . . . . . . . . . . . . . . . . 36

2.5 Numerical simulations . . . . . . . . . . . . . . . . . . . . 43

2.5.1 Nitrogen-vacancy center at room temperature - rate model . . . 43

2.5.2 Quantum optics toolbox . . . . . . . . . . . . . . 46

2.5.3 Validation of the rate model . . . . . . . . . . . . 48

2.5.4 Nitrogen-vacancy center at cryogenic temperatures . . . . . . . 48

3 Single nitrogen-vacancy center in a nanodiamond 53

3.1 Nanodiamonds on a mirror . . . . . . . . . . . . . . 55

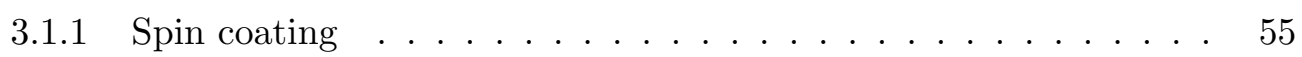

3.1.2 Confocal microscopy of the nanodiamonds on a mirror . . . . . 56

3.2 Intensity correlation of a single nitrogen-vacancy center . . . . . . . 60 
3.2.1 Relation between internal population dynamics and intensity correlation function . . . . . . . . . . . . . . 60

3.2.2 Measurements of the intensity correlation function of several different single nitrogen-vacancy centers . . . . . . . . . . 63

3.3 Fluorescence spectra of single nitrogen-vacancy centers . . . . . . . . . . 67

3.3 .1 Spectra at room temperature . . . . . . . . . . . 67

3.3.2 Spectra at cryogenic temperatures . . . . . . . . . . 71

4 Fiber resonators $\quad \mathbf{7 5}$

4.1 Fiber mirrors . . . . . . . . . . . . . . . . . 75

4.1.1 Structuring by laser machining . . . . . . . . . . . 76

4.1.2 Structuring by focused ion beam milling . . . . . . . . . . . 78

4.1.3 Further techniques . . . . . . . . . . . . . . . . . . . . . 80

4.1 .4 Choice of parameters . . . . . . . . . . . . . . . . 81

4.2 Technical aspects . . . . . . . . . . . . . . . . . . . 84

4.2.1 Background emission from the fiber . . . . . . . . . . . 84

4.2 .2 Mirror-coatings . . . . . . . . . . . . . . . . . . . . 87

4.2 .3 Building fiber cavities . . . . . . . . . . . . . . . . . 90

4.2 .4 Finesse . . . . . . . . . . . . . . . . . . . . 92

4.2 .5 Length stabilization schemes . . . . . . . . . . . . . 96

5 Single nitrogen-vacancy center coupled to a microcavity 103

5.1 Single nitrogen-vacancy center coupled to a plane mirror-fiber cavity . . 103

5.1.1 Identification of a certain nitrogen-vacancy center . . . . . . . 103

5.1.2 Spectrum and emission rate from the cavity-coupled nitrogenvacancy center . . . . . . . . . . . . . 105

5.1.3 Intensity correlation of the cavity emission . . . . . . . . . . . 107

5.1.4 Cavity tuning over several free spectral ranges . . . . . . . . . 109

5.1.5 Comparison to theoretical predictions . . . . . . . . . . . 112

5.2 Single nitrogen-vacancy center coupled to a fiber-fiber cavity . . . . . . 120

$\begin{array}{lll}6 & \text { Summary and future prospects } & 127\end{array}$

$\begin{array}{ll}\text { A Matlab codes } & 133\end{array}$

$\begin{array}{ll}\text { B Components } & 137\end{array}$

$\begin{array}{ll}\text { C Abbreviations } & 139\end{array}$

$\begin{array}{lr}\text { Bibliography } & 141\end{array}$

$\begin{array}{lc}\text { Publications } & 163\end{array}$ 


\section{Chapter 1}

\section{Introduction}

Observing and manipulating a single quantum system has become a routine in today's physics laboratories and has been the foundation for 2012 Nobel prize awards to Serge Haroche and David Wineland for their pioneering work in this field. A driving force behind all these experiments, beside the fundamental interest in directly observing non-classical physics, is the realization of a quantum computer [1], which relies on a single quantum two-level system, a quantum bit (qubit), as fundamental building block. Today's prospect of quantum information science (QIS) goes beyond Feynman's original idea of a quantum simulator and also includes very efficient algorithms for prime number factorization (Shor's algorithm) and for database searching (Grover's algorithm) [2] and furthermore theoretically absolute secure key distribution (quantum cryptography).

For realization of QIS many different systems ranging from single trapped atoms or ions, super-conducting circuits, single molecules, semiconductor quantum dots and single defects in solids, e.g. in diamond, are investigated. DiVincenzo formulated [3] five criteria (see below) which a physical system has to fulfill in order to be suitable as qubit. Fullfilling all of the criteria is a hard task in all the systems mentioned above. The bottleneck differs from system to system and no ideal system has yet been identified, such that the research on all the systems is still very active.

\subsection{The nitrogen-vacancy center in diamond}

One physical system that has witnessed a huge increase in interest over the past ten years is the nitrogen-vacancy (NV) center in diamond. It is a point defect in diamond consisting of a substitutional nitrogen atom and a neighboring vacancy and has first been reported by du Preez [4] in 1965. It's recent success is best visualized when looking at the number of publications in high-impact journals such as Science, Nature and Physical Review Letters, as displayed in Fig. 1.1. 1997 marks the year, when for the first time a single NV center was observed [5]. Since then, many important proof of principle experiments have been realized, which will be highlighted below. Even 
though many aspects of the electronic structure of the NV center have been known before, this recent increase in interest has also led to a very detailed understanding of the defect's properties, such that today it is clearly the best known defect in diamond. Its properties will be discussed in the next chapter.

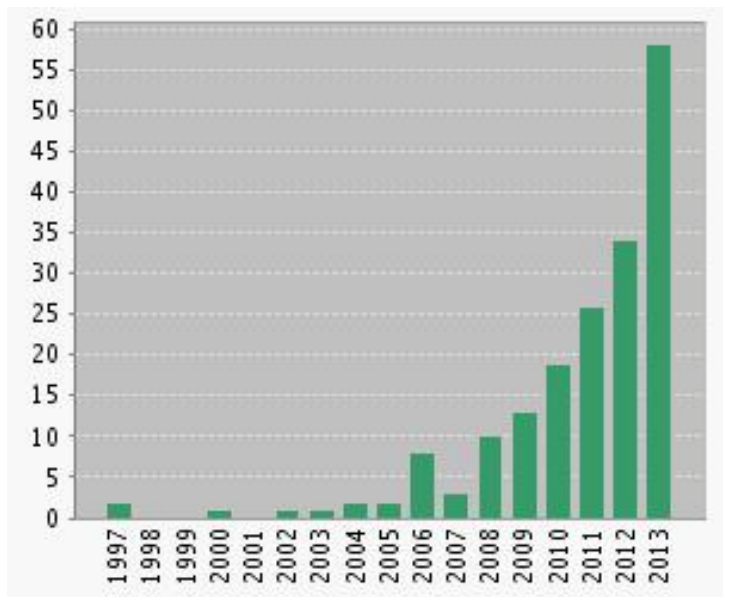

Figure 1.1: The annual number of publications on the topic of the nitrogen-vacancy center in Science, Nature, Nature Physics, Nature Photonics, Nature Communications, Nature Methods, Nature Materials, Nano Letters and Physical Review Letters. The graph has been created using the citation report function from Thomson Reuters' Web of Knowledge [6].

The first experiments observing a single NV center where performed with the focus to use it as a single photon source (SPS) [7-9], which enabled the successful demonstration of quantum key distribution $[10,11]$. However, for an application in quantum computing with photons, i.e. all optical quantum computing [12], one needs a SPS emitting indistinguishable photons. The spectrum of the emitted photons from the NV center is very broad and thus the NV center is per se not very suitable for this purpose. The emission is characterized by a zero phonon line (ZPL) at $638 \mathrm{~nm}$, i.e. the transition from the excited state to the ground state without creation of lattice vibrations of the diamond host and the phonon sidebands (PSB) at longer wavelengths ranging up to approximately $800 \mathrm{~nm}$, where, simultaneously with the emission of a photon, one or more optical phonons are created. The route to improve the spectral properties of the $\mathrm{NV}$ center is to couple it to a microcavity. This allows to alter the emission spectrum dramatically and therefore enables the use as SPS [13]. It is worth mentioning, that at cryogenic temperature a lifetime-limited linewidth of the ZPL has been measured [14]. However, this is not routinely measured due to lack of a perfect diamond crystal as host without strain and without further impurities.

The success of the NV center is due to some of its, for a solid state qubit, quite unique properties. Its ground state is a spin triplet state, which is split at zero magnetic field, into two spin sublevels $\left(m_{s}=0\right.$ and $\left.m_{s}= \pm 1\right)$, which form the basis for a 
qubit. When the NV center is optically excited the strength of fluorescence depends on the spin state allowing an optical readout of the spin state. Furthermore, off-resonant optical excitation also leads to spin polarization into one of the sublevels permitting the initialization of the qubit. Applying a microwave (MW) field coherently transfers the population between the two spin sublevels. This enabled the observation of Rabi oscillations at room temperature [15]. The coherence time is mainly limited by the presence of ${ }^{13} \mathrm{C}$ isotopes, which have a nuclear spin of $1 / 2$, whereas the spin of the most abundant isotope ${ }^{12} \mathrm{C}(98.9 \%)$ is zero. With advances in fabrication techniques diamond with very low concentration of ${ }^{13} C$ were grown, in which electronic spin coherence times $T_{2}>2 \mathrm{~ms}$ were observed $[16,17]$. This, for a solid state system at room temperature extremely long coherence times, were even observable for NV centers placed within $100 \mathrm{~nm}$ from the surface [18]. The observed dephasing times are on the order of $T_{2}^{\star}=100 \mu \mathrm{s}$, which are measured in contrast to the above mentioned coherence times $T_{2}$ without spin echo techniques. The spin coherence is preserved during optical excitation [19]. Due to strong hyperfine interaction within the excited state with the intrinsic spin of the nitrogen atom, the spin dephasing time in the excited state is much faster and on the order of the excited state lifetime (a few ns) [20]. Using the electronic spin, the realization of a quantum algorithm, the Deutsch-Jozsa algorithm, was shown [21].

Whereas coupling to a surrounding spin-bath limits the coherence time of the NV center, the hyperfine coupling of the electronic spin of the NV center to nuclear spins of neighboring ${ }^{13} \mathrm{C}$ atoms and/or the spin of a neighboring substitutional nitrogen atom $\left(I=1 / 2\right.$ for ${ }^{14} N$ and $I=1$ for $\left.{ }^{15} N\right)$ has proven to be a very powerful resource, that gives rise to two coupled qubits. Using radio-frequency $(\mathrm{RF})$ and $\mathrm{MW}$ fields, demonstration of the conditional two-qubit CROT gate was possible [22]. For short distances between the electronic spin and the nuclear spin the hyperfine coupling is strong enough, such that coherent dynamics between the two spins arise. This allows to initialize [23, 24] and to manipulate $[25,26]$ the nuclear spin via optically addressing the NV electronic spin. Using an NV center with two neighboring ${ }^{13} \mathrm{C}$ atoms and using the electronic spin as ancilla qubit any maximal entangled state between the two nuclear spins can be produced [27].

A CNOT gate with the nuclear spin as control and the electronic spin as target is implemented via application of a narrow-band nuclear spin state-selective MW pulse. Combined with subsequent repeated readout of the electronic spin, a projective singleshot readout of the nuclear spin is achieved and quantum jumps of the nuclear spins can be observed [28]. This is doable for the intrinsic nitrogen-spin of the NV center and a neighboring ${ }^{13} \mathrm{C}$ spin simultaneously [29]. The readout of the electronic spin can be enhanced by transferring the electronic spin to the nuclear spin via a CNOT gate with the electronic spin as control and the nuclear spin as target followed by repetitive readout of the nuclear spin as described above [30]. The nuclear spin is also very suitable as a quantum memory. Coherence times exceeding one second have been observed in isotopically purified diamond [31]. For this, the nuclear and electronic spin need to be decoupled, which can be achieved simply via continuous off-resonant excitation of the NV center [31]. This pumps the electronic spin out of the sublevels $m_{s}= \pm 1$ and 
additionally leads to fast de-ionization and ionization of the NV center.

A different readout technique makes use of spin-selective resonant optical transitions [32]. Due to slight spin mixing, resonant excitation also leads to shelving into the other spin state and thus additionally enables high fidelity spin state initialization. However, in contrast to all work described above, this technique requires cryogenic temperatures. Making use of this read-out method entanglement-by-measurement was shown between the spin of the intrinsic ${ }^{14} N$ and a close ${ }^{13} C$ nuclear spin [33]. All maximal entangled states were realized with high fidelity $(>90 \%)$ and for all states violation of Bell's inequality was shown [33].

A very fundamental result was the realization of a decoherence-protected quantum gate (at room temperature), which for any practical use of the NV center in a quantum computer is indispensable. Dynamical decoupling techniques, which decouple the qubit from the environment, normally also omit coupling between two qubits. This is useful to store the qubit, but not to process. A sophisticated pulse sequence is required to simultaneously decouple the qubits from the environment and performing a two qubit operation. For the NV center it is mainly the electronic spin which has to be protected. Van der Sar et al. [34] realized a controlled rotation gate (electronic spin as control, nuclear ${ }^{13}$ spin as target), where the electronic spin is decoupled from the environment. With their method, they further implemented Grover's quantum search algorithm with resulting state fidelity $>91 \%$. A slightly different approach was pursued by Xu et al. [35] using a continuous wave dynamical decoupling approach, where continuous illumination with two microwave fields resonant to the transitions from $m_{s}=0$ to $m_{s}= \pm 1$ (the degeneracy of the spin sublevels $m_{s}= \pm 1$ is lifted by an external magnetic field) projects the NV center into dressed states that are to first order insensitive to magnetic field fluctuations. However, in their proof-of-principle demonstration the prolonged coherence time came with the prize of much slower gate operation times.

The coupling between two individual NV centers has also been shown [28]. In order for the coupling to be strong enough, the NV centers need to be in close proximity $(<100 \mathrm{~nm})$. The orientation of the symmetry axes for the two NV centers were different, such that, with a magnetic field parallel to one of the NV center, the electronic spins could be individually addressed via MW fields at different frequencies. Entangling of the electronic spins was realized [36], which was combined with swap gates with the intrinsic nuclear nitrogen spin for longer storage times. For initialization and read-out optical access is needed. With a standard confocal microscope, which has been used in these experiments, the NV centers can not be addressed individually, as both lie within the diffraction limited spot of the microscope. In principle, techniques which are not bound by this limit, such as stimulated emission depletion (STED) microscopy [37] or ground state depletion (GSD) microscopy [38], have been shown to work for the NV center. However, maintaining the spin coherences is an issue because these techniques rely on switching off other close NV centers within the diffraction limited spot. In order to achieve this they are either optically excited and quickly de-excited (STED) or strongly saturated (GSD).

It is worth mentioning that all readout methods rely on detection of the fluorescence 
from the NV center. A limiting factor is the collection efficiency. As we will discuss later on, coupling to photonic structures or optical cavities can enhance the collection efficiency.

Beside the use as a qubit, the NV center has also proven to be very suitable for sensing applications. The NV center has a very narrow MW transition between the electronic spin sublevels. The transition frequency is sensitive to magnetic fields and is therefore a very sensitive magnetometer with a nanoscale resolution [39-41]. Attaching for instance a diamond nanocrystal $[42,43]$ or nanopillar [44] with a NV center to a atomic force microscope (AFM) tip, a scanning high resolution magnetometer can be realized. As diamond nanocrystals are also bio-compatible, it is even possible to track the orientation of a NV center inside a living cell, when making use of an external magnetic field [45]. Fabricating a microfluidic device on top of a shallow implanted (with nitrogen) diamond surface allows a sensitive detection of of the concentration of magnetic molecules such as manganese or ferritin by sensing their spin [46]. Further the NV center is sensitive to electric-fields [47] and it also can be used as sensor for temperature, as the magnetic resonance is temperature dependent $[48,49]$ and is detectable at temperatures up to $600 \mathrm{~K}[50]$.

For practical usage of the NV center as a qubit it has to fulfill the DiVincenzo criteria which are [3]: a suitably system has to be/have

1. a scalable physical system with well characterized qubits

2. the ability to initialize the state of the qubits to a simple fiducial state

3. long relevant decoherence times, much longer than the gate operation time

4. a "universal" set of quantum gates

5. a qubit-specific measurement capability

As discussed above, proof of principle experiments fulfilling criteria $2-5$ have been demonstrated. The critical point lies on the scalability in criterion 1. Proposals for scalable architectures [51] rely on a dense matrix of NV centers. Even though there has been huge progress in artificial creation of NV centers via ion implantation with $\mathrm{nm}$ spatial resolution [52-57], a deterministic creation of a dense array of single NV centers with adjacent ${ }^{13} \mathrm{C}$ atoms and their manipulation (in particular: individual addressing and manipulation without cross-talk) is still far beyond reach.

A second approach is a hybrid system, that combines different physical systems with different strengths. For instance, superconducting circuits allow very fast gate operation, whereas the NV center with its long coherence time is suitable as quantum memory. Strong coupling between an ensemble of NV centers and a superconducting resonator has been shown [58], where the superconducting resonator has been used in further experiments as bus to couple the spin ensemble to a superconducting qubit [59]. A second hybrid system with a NV center, that has been realized, is the coupling to a nanomechanical oscillator [60], where a diamond nanocrystal hosting a single NV 
center is attached to a silicon-carbite nanowire. A system, in which the nanomechanical oscillators couple via e.g. electrostatic forces is thinkable [61]. This could lead to a scalable system.

A very natural hybrid system is the combination of matter qubits (stationary qubits) and photons (flying qubits). Entanglement between two different matter qubits can be achieved via transferring first the internal degree of freedom of the matter qubit onto the photons and letting the photons interfere on a beam-splitter [62]. In challenging experiments quantum entanglement between an optical photon and the electronic spin of a NV center [63] and quantum interference of photons from different NV centers [64,65] have been shown. These experiments are challenging in the sense that they took a very long acquisition time (days) of data. Additionally, the achieved entanglement fidelity and contrasts in the interference experiments is very poor. The difficulties arise from the optical properties of the NV center's emission and might be overcome via coupling to an optical microcavity $[62,66]$.

\subsection{Cavity coupling}

The interaction between light and matter in general is weak and most of the time nothing happens, when a photon passes an atom because the interaction cross-section is small $\left(\approx \lambda^{2}\right)$. However, when the atom is placed inside an optical cavity, the interaction between photon and atom is increased, roughly speaking by the number of times the photons sees the atom, i.e. the finesse of the cavity. The effects of the cavity on the atom and vice versa are governed by the theory of cavity quantum electrodynamics (cavity QED) ranging from the weak coupling regime, where non-coherent processes dominate and the cavity is merely a perturbation for the atom's dynamics, to the strong coupling regime, where the coherent interactions exceed all other processes and cavity and atom can no longer be treated separately.

As identified above, there are three main aspects, where for the NV center coupling to a cavity plays an essential role: for use as SPS, for readout and to ensure scalability. In the weak coupling regime a cavity can increase the spontaneous emission rate via Purcell enhancement [67]. This effect will be discussed later in more detail (cf. Sec. 2.3.3) and is due to a change in the density of states of the vacuum field, to which the emission rate is proportional to. The decay is not only faster, but also preferably into a certain cavity mode. This is advantageous for the spin readout. With the higher emission rate and the spatial enhancement, which leads to a much higher collection efficiency, the NV center needs to undergo much less optical cycles between ground and excited states in order to discriminate between the spin sublevels. This reduces for instance the chances of nuclear spin-flips to occur.

The Purcell effect naturally is also very attractive to boost the performance of the NV center as SPS with a higher repetition rate, a higher collection efficiency and even a higher spectral performance [13].

As mentioned before, the commonly used readout of the spin state is achieved by 
measuring the fluorescence intensity. Besides the transition in the red spectral range there also exists a ZPL in the infrared (IR) associated with the NV center. Measuring the absorption on this frequency while simultaneously exciting the NV center allows discriminating between spin states of the NV center. This was used to implement a highly sensitive magnetometer [68]. However, it was necessary to operate the NV center at cryogenic temperatures. Placing the NV center inside a cavity, which is in resonance with the IR transition, will enhance the absorption and thus allow for spin readout by this method also at room temperatures [68].

There have been proposals for more elaborate schemes to readout the spin state and to entangle two different NV centers making use of a cavity. The basic idea is to have a cavity, which is only in resonance with the transition from one of the internal spin sublevels to the corresponding excited state. Depending on the state the NV center is in, the cavity either is highly reflective or highly transmissive for an incoming laser photon [69]. Preparing two NV centers in two different cavities each in a spin-superposition state and letting the photons reflected from the cavities interfere on a beam splitter allows to project the two NV centers into an entangled state [66]. Most schemes require the system to operate in the strong coupling regime to achieve correlations between the internal state of the matter qubit and the photon [70]. For a solid state system this is very challenging to realize. The above mentioned proposal [66] relaxes this constraint and thus reduces the demands on the cavity.

In short, cavity coupling is a very essential building block. Therefore a lot of attention has been given to this topic and many different implementations have been pursued in the last few years. There are two distinct approaches: first there is the hybrid approach, where the cavity is realized in non-diamond material and the NV center is incorporated into the cavity within a nanodiamond (ND) or a diamond film. Second, there are the all diamond systems where the optical cavity is realized within the diamond containing the NV center. The experiments have been both performed at room temperature and at cryogenic temperatures. The third aspect, in which the experiments differ, is the kind of cavity in use. There are three basic types: Fabry-Perot cavities consisting of two opposing mirrors, cavities based on whispering gallery modes such as ring resonators, microdiscs, microtorroids or microspheres and photonic crystal (PC) cavities based on a localized optical mode inside a photonic band gap material [71]. In the following an overview over these previous experiments is given sorted according to these categories.

1.) Hybrid approaches:

(a) Experiments at room temperature:

From an experimental point of view this is the easiest accessible regime and about half of the cavity experiments with NV centers fall into this category. Schietinger et al. $[72,73]$ positioned a polystyrene microsphere (diameter $4.84 \mu \mathrm{m}$ ) next to a ND on a cover slip containing a single NV center. They observed modes with quality factors $Q \approx 5000$ and with mode volumes $V_{\text {mod }}$ 
on the order of $10^{3}(\lambda / \mathrm{n})^{3}$. Larsson et al. [74] utilized a silica microsphere (diameter $50 \mu \mathrm{m}$ ) on top of diamond nanopillars containing an ensemble of NV centers. Despite their larger mode volume on the order of $10^{5}(\lambda / \mathrm{n})^{3}$ they achieve a much higher $Q / V_{\bmod }$ ratio due to a quality factor of $Q=2 \times 10^{6}$. With the help of a tapered fiber Barclay et al. [75] transferred a ND onto a glass microdisc (diameter $20 \mu \mathrm{m}$ ) and observed Fano resonances, i.e. the interference between the emission coupled first into the modes of the optical resonator and the directly collected emission. With $Q=1.7 \times 10^{5}$ and $V_{\text {mod }}=86(\lambda / \mathrm{n})^{3}$ they achieve a $Q / V_{\text {mod }}$ ratio comparable to experiments using PC cavities (see below) which is one order of magnitude larger than the experiments of Larsson et al. [74]. Gregor et al. [76] performed the same experiment using the same transferring technique with a glass microtorroid (diameter $65 \mu \mathrm{m}$ ). Their slightly higher Q-factor of $Q=5 \times 10^{5}$ came with a significantly larger mode volume and thus worse $Q / V_{\text {mod }}$ ratio. In both experiments the ND contained multiple NV centers. In a further experiment, instead of using NDs, Barclay et al. [77] directly fabricated a gallium phosphide $(\mathrm{GaP})$ microdisc (diameter $4.5 \mu \mathrm{m}$ ) on top of a diamond bulk crystal with quality factor $Q=1.7 \times 10^{4}$ and mode volume $V_{\text {mod }}=18(\lambda / \mathrm{n})^{3}$.

A couple of experiments have employed GaP PC cavities. Englund et al. [78] moved the GaP slab over a diamond film and positioned the cavity on top of a single NV center. This is the only experiment were for an NV center at room temperature coupled to a cavity a change in lifetime (from $16.4 \mathrm{~ns}$ to $12.7 \mathrm{~ns}$ ) was actually measured. Using a pick-and-place technique [79] a ND containing a single NV center was deposited on top of the PC cavity by Wolters et. al [80]. In a similar experiment, applying a slightly different pick-and-place method [81], van der Sar et al. dropped a ND into the hole of the PC structure [82], where the cavity was designed to yield a mode with high intensity inside the air hole. The mode volume for all the PC cavities were on the order of $1(\lambda / n)^{3}$ and quality factors Q between $500-1600$. In these experiments the emission into the cavity mode is enhanced by a factor on the order of $F=10$.

Common to all these experiments is the observation of cavity resonances on top of the NV spectrum, i.e. they simultaneously detect directly emitted light from the NV center and light first coupled to the modes of the cavity. The cavity modifies the local density of states and therefore changes the emission rate at the cavity wavelength into the mode.

A ND with a single NV center inside a Fabry-Perot cavity with two plane mirrors has been used by Dumeige et al. [83] in their experiments. They observed a broad resonance $\delta \lambda=22 \mathrm{~nm}$. Comparing the emission rate into the cavity mode with the emission rate into free space, they observed an increased spectral density of emission by a factor of 2 and hence demonstrated coupling of the NV center to the cavity mode. 
(b) Experiments at cryogenic temperature:

The earliest cavity QED experiment with NV centers [84] has revealed some quite remarkable results. The NDs were dispersed onto a silica microsphere and the coupling between the NV center emission and a whispering gallery mode was investigated at cryogenic temperatures. While tuning the resonance frequency by varying the temperature, they observed an anti-crossing between cavity and NV center's emission frequency and are thus in a strong coupling regime. This is the only experiment up to date where this regime has been achieved. Even though many NDs were dispersed on the microsphere, the observed cavity mode is expected to be coupled only to a single $\mathrm{NV}$ center. This is due to the inhomogeneous distribution of NV center's ZPL frequency in NDs and the fact, that only very few NV centers are spectrally stable enough to remain within the narrow cavity resonance of the high Q cavity mode of the microsphere. However, despite many efforts in this field, the strong coupling regime for a single NV center could not been reached in any other experiments and there are doubts, whether in the above mentioned experiment only a single NV center was involved, as this has not been proven unambiguously.

The system using diamond nanopillars and a microsphere positioned on top of the pillars was not only investigated at room temperature [74] but also investigated at $150 \mathrm{~K}$ [74]. However, in their proof-of-principle demonstration they only show a modulated spectrum by the cavity peaks. Using GaP microdiscs or microrings on top of a diamond sample two experiments $[85,86]$ show a slight reduction in lifetime of the NV center ZPL emission coupled to the microcavity at cryogenic temperatures ( $10 \mathrm{~K}$ or $6 \mathrm{~K}$, respectively) which is attributed to a Purcell factor in the range $F_{p}=3-6$. Both these experiments were performed with ensembles of NV centers.

2.) All-diamond systems:

The fabrication of photonic structures inside diamond is challenging, since no established technique for high-precision machining of diamond exists. In early experiments optical resonances of a microdisc [87] and of PC cavity [88], which were fabricated by dry-etching of a nanocrystalline diamond film, were demonstrated. A second method was the use of focused ion beam milling to fabricate for instance nanobeam PC cavities [89]. However, these first structures did not show any optical modes. By post-annealing to counter the damage induced by the focused ion beam, PC cavities having optical resonances with quality factors in the range $500-1000$ were fabricated [90]. The coupling to an ensemble of silicon-vacancy $(\mathrm{SiV})$ centers (another color center in diamond consisting of a vacancy neighboring a substitutional silicon atom where the silicon atom relaxes towards the vacancy leading to the so called split vacancy configuration [91]) was 
demonstrated and an enhancement of the ZPL line intensity by a factor of 3 was achieved. Working at cryogenic temperatures, Faraon et al. [92] showed an enhancement of the ZPL emission of individual NV centers into a diamond ring resonator by a factor of 10 . The resonator was fabricated by etching a thin diamond membrane, that had been bonded onto a silicon substrate. They reported in later work [93] an even larger enhancement into a PC cavity, fabricated in the same manner, observing a change in lifetime from $13.7 \mathrm{~ns}$ down to $4.0 \mathrm{~ns}$ and $70 \%$ of the emission channeled into the ZPL, whereas for a non-coupled NV center the ratio between emission into the ZPL and the PSB for an NV center is only approximately 3\%. Further steps towards all-diamond networks with a diamond microring resonator containing a single NV center, which is coupled to a diamond waveguide, have been demonstrated [94,95].

\subsection{Scope of this thesis}

This thesis contains both the theoretical framework to describe the coupling of a single NV center to a microcavity and its experimental realization at room temperature using Fiber-based cavities.

In Chap. 2 first all the relevant theoretically aspects of the NV center are presented. Second, an overview of cavity QED is given before the special case of an NV center coupled to a cavity is discussed. The last part of the chapter contains the numerical simulation to predict the cases of a NV center coupled to a microcavity both at room temperature and at cryogenic temperature.

We use single NV centers in NDs that are spin coated onto a mirror. Details of the sample preparation and the experimental characterization of these NV centers including emission spectra and the internal population dynamics deduced from intensity correlation measurements are discussed in Chap. 3.

We employ fiber-based Fabry-Perot microcavities, which are based on concave mirrors fabricated on fiber facets. In Chap. 4 first the fabrication process of these fiber mirrors is illustrated and the choice of parameter range for the concave structure is discussed. In the second part several technical aspects concerning fiber-based cavities, such as background fluorescence from the fibers, deposition of the mirror coatings, realization and characterization of fiber cavities and schemes to stabilize the cavity length are presented.

In Chap. 5 we discuss the actual experiments, where a single NV center in a ND is coupled to a fiber-based microcavity. Two different kinds of cavities, first a cavity with one fiber mirror and one standard plane mirror and second a cavity consisting of two fiber mirrors are used. The obtained results are compared to the prediction made by the theoretical model introduced in Chap. 2.

The thesis concludes with Chap. 6 summarizing the work and giving an outlook to future prospects and experiments. 


\section{Chapter 2}

\section{Theoretical background}

This chapter describes the theoretical background for this work. Coupling of a single NV center to a microcavity establishes the integration of a solid state emitter with cavity QED. Both realms will first be introduced independently, before the theory and simulations of the interplay of the two are shown. In the first section today's knowledge of the NV center is displayed, which follows mainly a recent review from M. Doherty et al. [96]. The second part contains first a general overview over Fabry-Perot cavities followed by a description of the fundamentals of cavity QED. In the final sections the theoretical model for the concrete case of a single NV center coupled to a microcavity is introduced and employed in numerical simulations to predict the experimental results.

\subsection{Nitrogen-vacancy center in diamond}

\subsubsection{Overview}

The NV center was first reported by L. du Preez in 1965 [4]. In the following years many insights into the structure and electronic configuration of the center were gained. The demonstration of single photon emission from a single NV center $[5,7,8]$ had a huge impact, since it opened the possibility of manipulating a single quantum system, which has led to huge growth of interest in the NV center and a very detailed understanding of the defect's properties.

This first part will sketch a general picture of the NV center with its key properties. In the second subsection, following the historical development, details on the electronic structure are given. Afterward the focus lies on the vibronic and optical properties of the defect, which to know is essential when describing the coupling to an optical cavity later on.

The NV center is a point defect in the diamond lattice called a color center consisting of a substitutional nitrogen atom $(\mathrm{N})$ and a adjacent vacancy $(\mathrm{V})$ as shown in Fig. 2.1. It is one of the most common defects in diamond. NV centers are found in nat- 
ural and synthetic [chemical vapor deposition (CVD) or high-pressure-high-temperature (HPHT)] diamonds. They can also be produced artificially in $1 \mathrm{~b}$ diamond containing a low concentration of substitutional nitrogen by creation of vacancies, via for example electron irradiation, followed by annealing at temperatures $>700^{\circ} \mathrm{C}[97,98]$ or in ultra-pure diamond by nitrogen implantation and subsequent annealing [52-57].

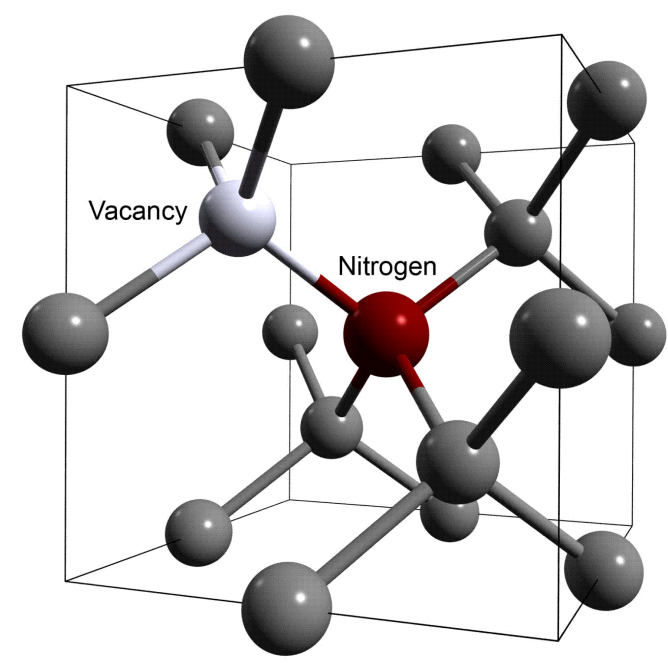

Figure 2.1: Atomic structure of the NV center consisting of a substitutional nitrogen atom and a adjacent vacancy.

The NV center exists in two different charge states: neutral $\left(\mathrm{NV}^{0}\right)$ and negatively charged $\left(\mathrm{NV}^{-}\right)[99,100]$. The main interest lies on the negatively charged $\mathrm{NV}^{-}$center and thus when not specified explicitly I am referring to the $\mathrm{NV}^{-}$. Both charge states have discrete energy levels within the diamond bandgap leading to sharp optical zero phonon lines (ZPLs) at $637 \mathrm{~nm}(1.945 \mathrm{eV})$ for $\mathrm{NV}^{-}$[4] and at $575 \mathrm{~nm}(2.156 \mathrm{eV})$ for $\mathrm{NV}^{0}$ [101]. The difference in the ZPL wavelengths allows discrimination between the two charge states.

The ground and excited state of the $\mathrm{NV}^{-}$center are spin triplet states. At zero magnetic field the ground state is split into the $m_{s}=0$ and $m_{s}= \pm 1$ sub-level by $2.88 \mathrm{GHz}$ [99]. The excited state has a lifetime of $12.9 \mathrm{~ns}$ [102]. There are two intermediate singlet states, which are linked by a fast (lifetime $0.9 \mathrm{~ns}$ ) optical transition in the infrared [103]. The lower of these two singlet states is a metastable state with a longer lifetime (300-460 ns [104-106]). The metastable singlet states decays to both $m_{s}=0$ and $m_{s}= \pm 1$ ground states. However, nearly only the $m_{s} \pm 1$ excited state decays to the intermediate singlet states, where the electron will be "shelved", which results in less fluorescence. This allows to optically detect the spin state of the NV center. Therefore, the $m_{s}=0$ state is often referred to as bright state, whereas the $m_{s}= \pm 1$ state is called dark state. Furthermore, off-resonant optical excitation, which 
is spin conserving, also leads to spin polarization into the $m_{S}=0$ sub-level $[15,107,108]$. Figure 2.2 summarizes these key features of the negatively charged NV center.

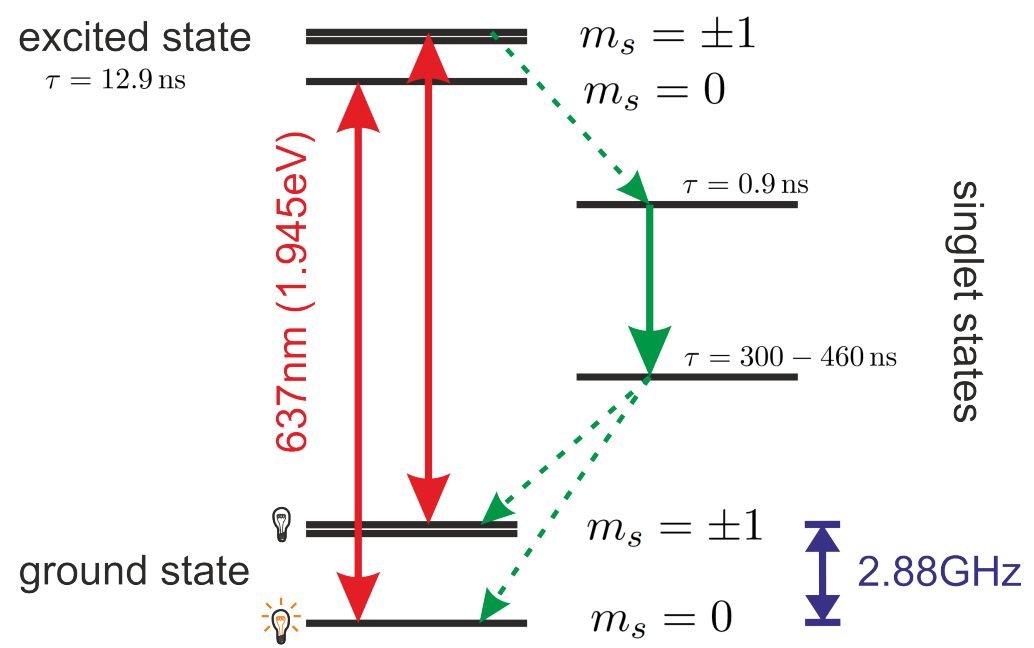

Figure 2.2: Simplified energy level scheme for the negatively charged NV center with allowed transition.

Because the NV center is hosted in the diamond matrix, it also couples to phonons. Emission is not only observed at the ZPL wavelength but also at longer wavelengths. The fluorescence spectrum in fact is dominated by these so called phonon sidebands (PSBs) as shown in Fig 2.3 displaying the emission spectrum from a single NV center. The same is also true for absorption, where not only at the ZPL wavelength absorption is observed, but also at shorter wavelengths.

\subsubsection{Electronic structure}

The discussion here is limited to the negatively charged NV center. After first observation of the NV center by du Preez [4], Davies et al. established the symmetry of the defect with unilateral stress experiments as $C_{3 v}$ [97]. The NV structure, as already correctly suggested by du Preez [4], was confirmed by electron paramagnetic resonance (EPR) measurements by Loubser and van Wyk [99]. Attributing their signals to a ${ }^{3} \mathrm{~A}$ triplet state, they also proposed the NV center to be negatively charged, which eventually was confirmed unambiguously by Mita et al. [100], observing the concentration change between neutral charge state and negatively charge state after neutron irradiation. The presence of this triplet state was explained by six unpaired electrons within the defect, 3 electrons from the dangling bonds of the surrounding carbon atoms, 2 unpaired electrons from the nitrogen atom and 1 electron as extra charge. From symmetry arguments the molecular orbitals (MOs) occupied by these 6 electrons were deduced as two $A_{1}$ and one degenerate $E$ MOs. Filling these MOs with the 6 electrons led, in the lowest energy configuration, to a triplet ${ }^{3} A$ level and two singlet levels. Loubser and 


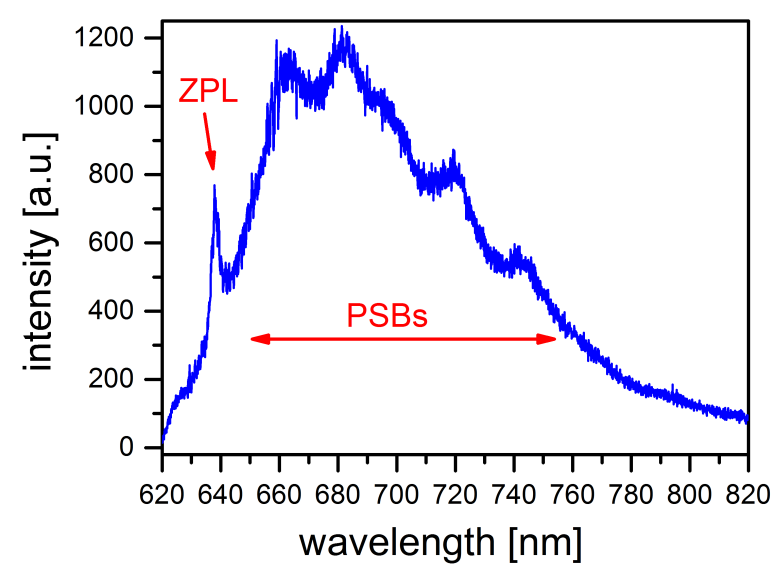

Figure 2.3: Fluorescence spectrum of a single NV center showing the zero phonon line (ZPL) at $\approx 640 \mathrm{~nm}$ and the phonon side bands (PSBs).

van Wyk wrongly assumed the ${ }^{3} A$ triplet state as a metastable state and the optical transition to occur between the two singlet states, since they did not observe any EPR signal without optical illumination.

Two laser spectral hole burning experiments by Reddy et al. [109] and optically detected magnetic resonance (ODMR) by van Oort et al. [107] later on revealed the triplet nature of the ground state. This was confirmed by detection of EPR in the dark, i.e. without optical illumination $[110,111]$. The correct ordering between the $m_{s}=0$ and $m_{s}= \pm 1$ sub-levels was established by Raman heterodyne paramagnetic resonances $[112,113]$. With EPR spectroscopy at low temperature Harrison et al. [108] showed that laser illumination leads to spin polarization into the $m_{s}=0$ sub-level.

The theoretical description of the energy levels and different interactions was under constant debate and some issues still remain to be answered. Pioneering work was done by Lenef and Rand [114], who applied linear combinations of atomic orbitals. They constructed the wave functions respecting the $C_{3 v}$ symmetry of the NV center for the 2, 4 and 6 electron case and evaluated the spin-orbit, spin-spin, strain and Jahn-Teller interactions. However they falsely concluded the 2 electron neutral model to be the correct case for the NV center, which already at the time was believed to be wrong [115]. In a series of works the 6 electron model has been well established [104,116-119]. Huge insights came from the measurements determining the excited state structure at low temperature $[120,121]$ and at room temperature $[122,123]$ and the theoretical understanding of the room temperature measurements to result from time-averaging effects within the excited state [124]. A second point of long controversy was the numbers of singlet levels between ground and excited state and their ordering. With experimental demonstration of a second ZPL at $1046 \mathrm{~nm}$ [103] two singlet levels, which lifetimes have also been measured later on [105], have been established to lie between ground and 
excited state. The ordering of the two singlet levels, as derived from density functional theory (DFT), has been in contradiction to the mechanism believed responsible for spin polarization [125,126]. Manson et al. [127] showed experimental evidence for the ordering calculated by DFT to be true and give a theoretical explanation for the optically induced spin polarization mechanism. The details however are still under debate.

In the following the electronic structure of the NV center as known up to now is illustrated. From the $C_{3 v}$ symmetry the molecular orbitals which are occupied by the 6 electrons of the NV center are deduced. There are two " $a_{1}$ " orbitals, one of them within the valence band, and a degenerate " $e$ " orbital as illustrated in Fig. 2.4(a) $[117,118]$. The lowest energy configuration is achieved by 4 electrons occupying the $a_{1}$ orbitals, 2 electrons in each, and the two remaining electrons in the $e$ orbital. In this configuration there exist two singlet states ${ }^{1} A$ and ${ }^{1} E$ and a triplet state ${ }^{3} A_{2}$ where the triplet state is lowest in energy. By promoting an electron from the $a_{1}$ orbital to the $e$ orbital the energetically next higher configuration is reached. In this configuration there is a ${ }^{3} E$ triplet state which corresponds to the excited state of the NV center and an energetically higher singlet state ${ }^{1} E$, which for the main properties of the NV center is not relevant. There are two singlet states in between ground and excited state. The states formed from the electronic configurations are shown in Fig. 2.4(b) [117,118].

(a)

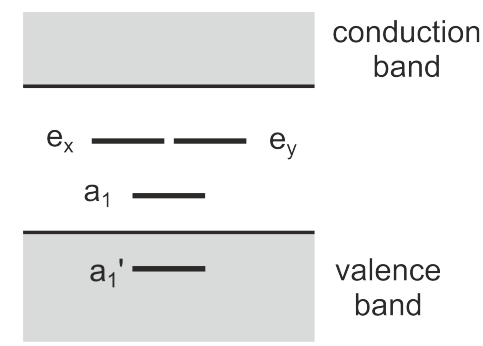

(b)

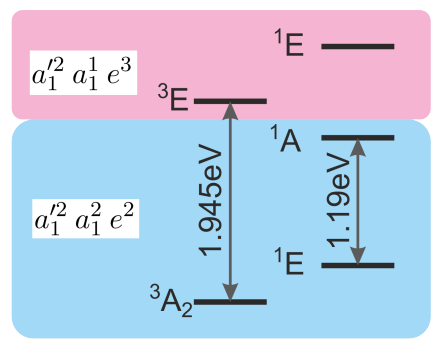

Figure 2.4: (a) Molecular orbitals respecting the $C_{3 v}$ symmetry of the NV center which are occupied by the 6 electrons of the $N V^{-}$. (b) Possible states deduced from the electronic configuration with the correct energetic ordering. The ground state of the NV center is the ${ }^{3} A_{2}$ triplet state. The excited state corresponds to the ${ }^{3} E$ triplet state. There are two singlet states between the ground and excited state.

The fine structure of the ground and excited state is determined by spin-orbit, spinspin and strain interactions $[117,118]$. Figure 2.5(a) illustrates the effect of the different interactions on ground and excited state: Spin-orbit interactions in the ground state is negligible, whereas spin-spin interaction leads to a splitting between the $m_{s}=0$ and $m_{s}= \pm 1$ sub-level. The sub-levels of the ground state are not affected by a strain field. In the excited state spin-orbit interaction leads to a splitting into 3 different sub-levels, two with $E$ symmetry and one with $A_{1}, A_{2}$ symmetry. These sub-levels are shifted further by spin-spin interaction and the degenerate $A_{1}, A_{2}$ symmetry state is split. Hence without strain the excited state consists of 4 sub-levels with $A_{1}, A_{2}$ and 
twice $E$ symmetry. Note that the $E$ symmetry states are degenerate. Strain will lift this degeneracy and when the strain is dominant over the spin-spin interactions the excited state is split into two triplets with $E_{x}$ and $E_{y}$ symmetry, whose degeneracies are lifted by spin-spin interactions. Investigating different NV centers in different environments, Batalov et al. [121] were able to measure the effect of different strain on the excited state level structure [see Fig. 2.5(b)].

(a)
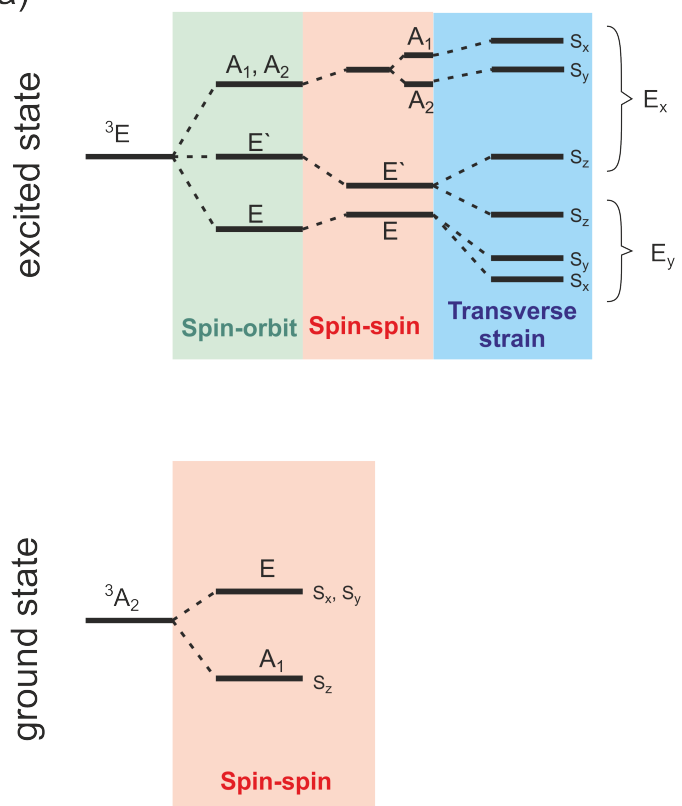

(b)

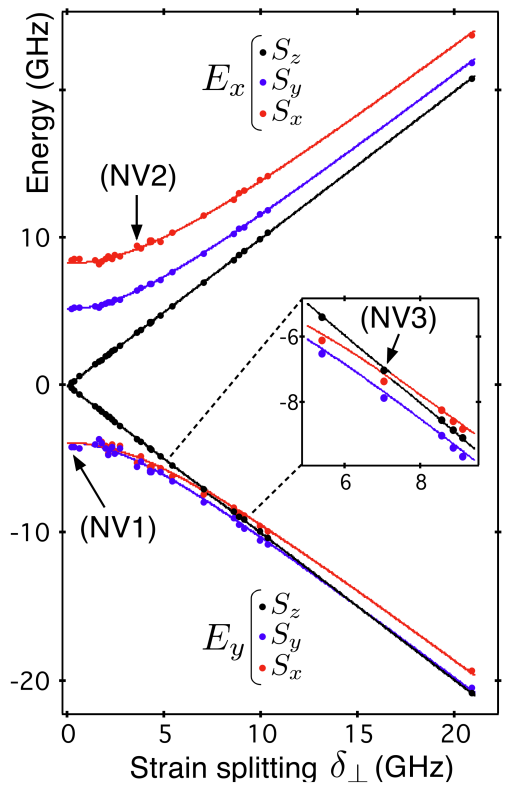

Figure 2.5: (a) Effect of the spin-orbit, spin-spin and strain interactions on ground and excited state [121]. (b) Measurement and theoretical prediction of excited state level structure as function of lateral strain. Reprint from [121]. Copyright (2009) by The American Physical Society.

At room temperature electron spin resonance (ESR) measurements [122,123] showed only a single resonance in the excited state at $1.43 \mathrm{GHz}$ which is split in a magnetic field. This comes from time-averaging at ambient temperatures within the excited state of the $A_{1}, A_{2}$ and $E$ level due to interactions with phonons as was shown by Rogers et al. [124] (see Fig. 2.6). The observed resonance is between the effective time-averaged level and the $E^{\prime}$-level of the excited state. Due to this averaging, the excited state magnetic zero field splitting is also independent of any strain in the crystal.

Describing most experimental situations for the NV center, the two singlet states between ground and excited state can be treated as a single intermediate state because they are linked by a fast transition. This allows describing the NV center's dynamics at room temperature by an effective 5 level model as shown in Fig. $2.6[106,127]$. The relative strengths of the inter-system crossing have been measured by Manson et 
al. [127] and Robledo et al. [106]. The excited state sublevels have different lifetimes: For the $m_{s}=0$ sublevel the lifetime is $\tau_{m_{s}=0}=13.7 \mathrm{~ns}$ and for the $m_{s}= \pm 1 \tau_{m_{s}= \pm 1}=$ $7.3 \mathrm{~ns}[106,128]$.

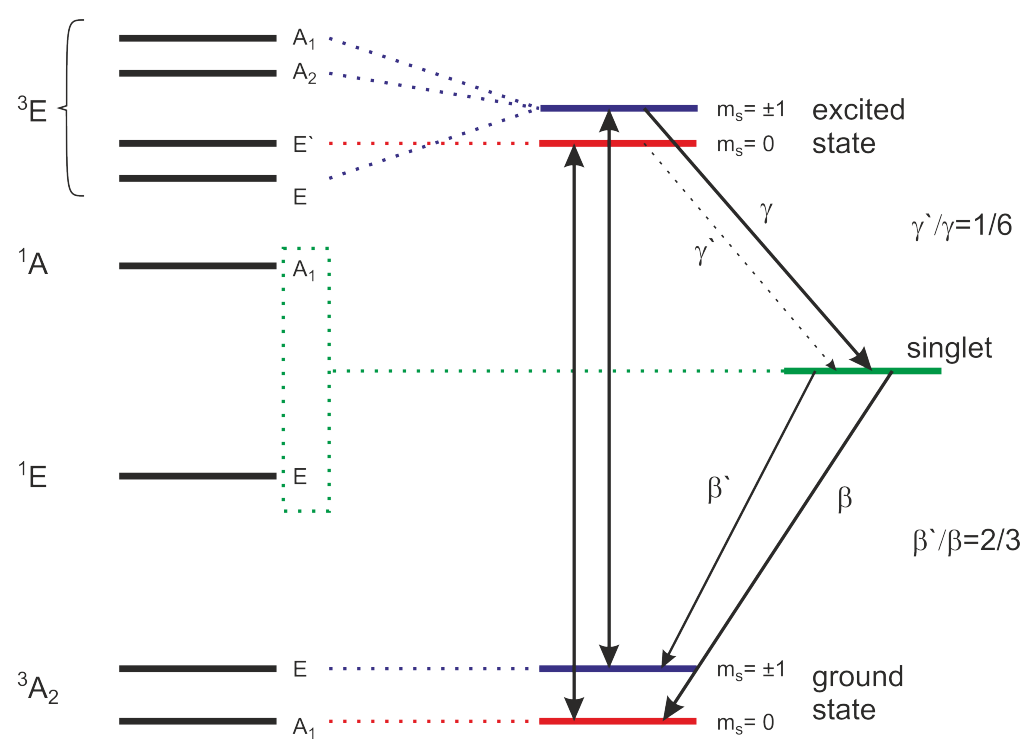

Figure 2.6: Effective 5 level model for the NV center at room temperature with one intermediate state and ground and excited state split at zero magnetic field into $m_{s}=0$ and $m_{s}= \pm 1$ sub-levels.

Interactions with the nuclear spin of the ${ }^{14} N$ or ${ }^{15} N$ nitrogen atom or with the nuclear spin of a neighboring ${ }^{13} C$ atom will lead to hyperfine splitting within the ground state which has been used to entangle electron and nuclear spin [15] or two nuclear spins $[27,33]$.

\subsubsection{Vibronic structure and optical properties}

\section{Phonon side bands}

The vibronic band of the NV center is approximated rather well by the Huang-Rhys model [129]. A single vibrational mode is used to treat the quasi-continuum of phonons of the diamond lattice. The normal coordinate of this mode correspond to the displacement of the neighboring nuclei. Using the Born-Oppenheimer approximation ground and excited state are described by harmonic (as a further approximation) potentials in the configuration space of the nuclear coordinates with discrete vibrational energy ladders. The energy minima for ground and excited state are not at the same nuclear configuration but are shifted by $\delta Q$ in the normal coordinate space as sketched in Fig. 2.7. The Franck-Condon principle states that the most likely transitions are at the same nuclear configuration, i.e. the vertical ones in Fig. 2.7. But the other transitions 
are also allowed as the overlap of the vibrational wave functions is non-zero and the probability is given by the Franck-Condon factors.

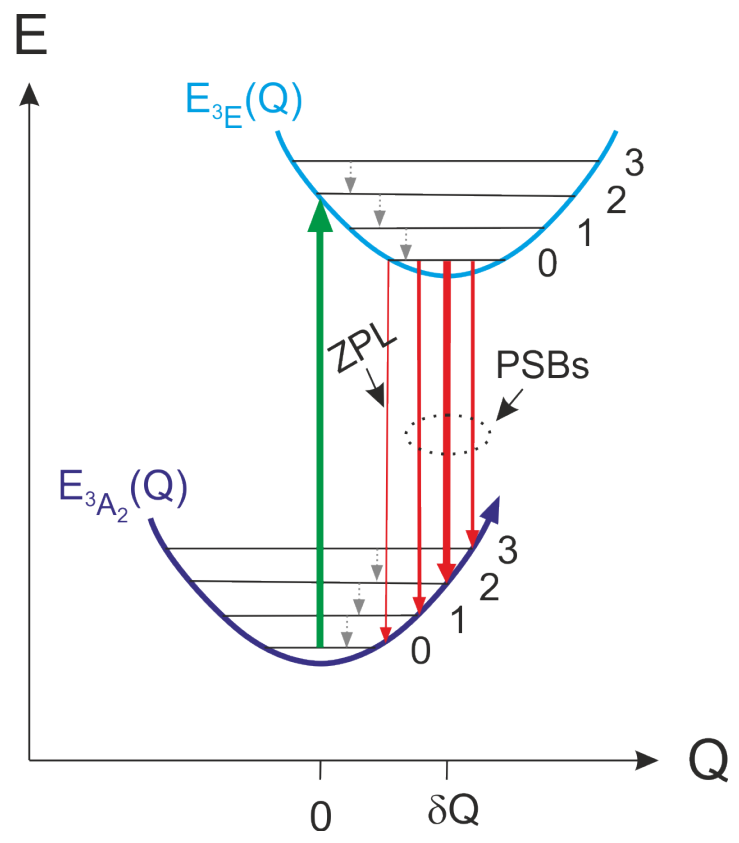

Figure 2.7: Huang-Rhys model in the Franck-Condon approximation. Ground and excited state are described by a harmonic potential in the nuclear configuration space and form a ladder of vibrational levels. The most likely transition are vertical i.e. between states with the same configuration coordinate $Q$ [129].

The quadratic potentials for the ground and excited state as function of the nuclear coordinates have the form [130]:

$$
\begin{aligned}
E_{g}(Q) & =\frac{1}{2} m \omega^{2} Q^{2}, \\
E_{e}(Q) & =E_{e}+\frac{1}{2} \omega^{2} Q^{2}+a Q+b Q^{2} \\
& =E_{e}-E_{R}+\left(\frac{1}{2} m \omega^{2}+b\right)(Q-\delta Q)^{2},
\end{aligned}
$$

where $\mathrm{a}$ and $\mathrm{b}$ are the linear and quadratic electron-vibration coupling parameters of the excited state. The frequency of the vibrational mode in the ground state $|g\rangle$ is $\omega$ and in the excited state $|e\rangle \Omega=\sqrt{\omega^{2}+2 b / m}$. In diamond frequency changes are small and can often be neglected [130]. The displacement of the excited state in the vibrational coordinates with respect to the equilibrium configuration of the ground state is given by $\delta Q=-a /\left(m \omega^{2}+2 b\right)$. The reduction of energy resulting from this changed nuclear configuration is the relaxation energy $E_{R}=a^{2} /\left(2 m \omega^{2}+4 b\right)$. 
To calculate transition probabilities for optical transition one has to evaluate integrals of the form

$$
\iint d r d Q \phi_{e}^{\star}(r) \chi_{n}^{\star}\left(Q-Q_{0}\right) D \phi_{g}(r) \chi_{0}(Q),
$$

where $\mathrm{D}$ is the optical dipole operator and the wave function is assumed as product of electronic and nuclear part, i.e. for the ground state: $\psi(r, Q)=\phi_{g}(r) \chi_{0}(Q)$. In the Condon approximation the integral over the electronic part is assumed to be independent of the vibrational coordinate such that the integral factorizes:

$$
\int d r \phi_{e}^{\star}(r) D \phi_{g}(r) \int d Q \chi_{n}\left(Q-Q_{0}\right) \chi_{0}(Q)
$$

Defining the Franck-Condon factors as:

$$
M_{0 n}=\int d Q \chi_{n}\left(Q-Q_{0}\right) \chi_{0}(Q)
$$

the harmonic states give

$$
\left|M_{0 n}\right|^{2}=\frac{e^{-S} S^{n}}{n !} \quad \text { where } \quad S=a^{2} / 2 m \hbar \omega^{3} \equiv E_{R} / \hbar \omega .
$$

$S$ is the Huang-Rhys factor which measures the fraction of the optical transition which lies in the ZPL:

$$
S=\log \left(\frac{\sum_{n=0}^{\infty}\left|M_{0 n}\right|^{2}}{\left|M_{00}\right|^{2}}\right)
$$

For the NV center the Huang-Rhys factor is 3.76 and the energy of the vibrational mode is $\hbar \omega=65 \mathrm{meV}$ [130]. The maximum in the emission spectrum is approximately at a frequency of $\nu_{\max }=\nu_{\mathrm{ZPL}}+S \times \hbar \omega$.

The Huang-Rhys model is a strong simplification with the assumption of single vibrational frequency. To go beyond one has to include many modes. For linear electron coupling with $\mathrm{N}$ modes the energy levels are [130]

$$
\begin{aligned}
E_{g} & =\frac{1}{2} \sum_{i=1}^{N} m_{i} \omega_{i}^{2} Q_{i}^{2}, \\
E_{e} & =E_{e}+\frac{1}{2} m_{i} \omega_{i}^{2} Q_{i}^{2}+\sum_{i=1}^{2} a_{i} Q_{i} \\
& =E_{e}+\frac{1}{2} m_{i} \omega_{i}^{2}\left(Q_{i}+a_{i} /\left(m_{i} \omega_{i}^{2}\right)\right)^{2}-\underbrace{\frac{1}{2} \sum_{i=1}^{N} a_{i} /\left(m_{i} \omega_{i}^{2}\right)}_{E_{R}} .
\end{aligned}
$$

The Huang-Rhys factors are defined as

$$
s_{i}=a_{i}^{2} /\left(2 \hbar \omega_{i}^{3} m_{i}\right) \quad \text { and } \quad S=\sum_{i=1}^{N} S_{i} .
$$


The one-phonon sideband consists of a transition, where one of the $\mathrm{N}$ vibrational modes is excited. The Franck-Condon factor for the case where the $i$ vibrational mode is excited is:

$$
\begin{aligned}
M_{01}^{i}= & \int d Q_{i} \chi_{1}^{0 \star}\left(Q_{1}\right) \chi_{1}^{0}\left(Q_{1}-Q_{1}^{0}\right) \ldots \int d Q_{i} \chi_{i}^{0 \star}\left(Q_{i}\right) \chi_{i}^{1}\left(Q_{i}-Q_{i}^{0}\right) \\
& \times \ldots \int d Q_{N} \chi_{N}^{0 \star}\left(Q_{N}\right) \chi_{N}^{0}\left(Q_{N}-Q_{N}^{0}\right), \\
\left|M_{01}^{i}\right|^{2}= & S_{i} \exp \left(-S_{i}\right) \prod_{j \neq i} \exp \left(-S_{j}\right)=S_{i} e^{-S} .
\end{aligned}
$$

The total relative intensity on the one-phonon band is thus

$$
\left|M_{01}\right|^{2}=\sum_{i}\left|M_{01}^{i}\right|^{2}=S e^{-S}
$$

and similar for the $\mathrm{n}$-phonon band, where $\mathrm{n}$ phonons are created in any of the $\mathrm{N}$ modes

$$
\left|M_{0 n}\right|^{2}=\frac{S^{n} e^{-S}}{n !} .
$$

Note that the expressions here look formally the same as for a single vibrational mode. It is sufficient to know the spectral shape of the one-phonon sideband, because for the n-phonon band the shape can be calculated recursively:

$$
I_{n}(\nu)=\int_{0}^{\omega_{m}} d x I_{1}(x) I_{n-1}(\nu-x),
$$

where $\omega_{m}$ is the cut-off frequency of vibrational modes supported by the crystal and $I_{1}$, $I_{n-1}$ the spectral form of the one-phonon or (n-1)-phonon band, respectively. By fitting the spectrum of the NV center, Davies [131] determined the shape of the one-phonon band for the NV center which is sharply peaked at around $65 \mathrm{meV}$. This explains why the Huang-Rhys model, i.e. the assumption of dominant coupling to one particular phonon mode, works well for the NV center. In recent publications a more precise picture of the vibrational modes of the NV centers has been developed: Discrete vibrational energy levels have been calculated by ab initio studies [132-134] and also experimentally measured via two-dimensional electronic spectroscopy [135].

\section{Line broadening mechanism of the ZPL}

There are two main contributions to line broadening of the ZPL: dephasing due to Jahn-Teller interactions $[133,136]$ and spectral diffusion because of a varying Stark shift associated with change in the static electric fields due to ionization of nearby impurities such as substitutional nitrogen [14]. 
The Jahn-Teller effect occurs in degenerate levels, as it is the case for the orbitally degenerate ${ }^{3} E$ excited state of the NV center. This level couples to a doubly degenerate $e$ vibrational mode. The interaction is governed by the Hamiltonian [133]

$$
\begin{aligned}
H^{\mathrm{el}}\left(Q_{x}, Q_{y}\right)=E_{0} & +\frac{K}{2}\left(Q_{x}^{2}+Q_{y}^{2}\right) \sigma_{z}+F\left(Q_{x} \sigma_{z}-Q_{y} \sigma_{x}\right) \\
& \left.+G\left[\left(Q_{x}^{2}-Q_{y}^{2}\right) \sigma_{z}+2 Q_{x} Q_{y} \sigma_{x}\right)\right]
\end{aligned}
$$

where $\sigma_{x}$ and $\sigma_{z}$ are Pauli matrices and $Q_{x}$ and $Q_{y}$ are the displacement coordinates for the $e$ vibrational mode in $C_{3 v}$ symmetry. $\mathrm{K}$ is the elastic force constant; $\mathrm{F}$ and $\mathrm{G}$ are the linear and quadratic vibronic constants. Similar to the Huang-Rhys model the energy minimum in the excited state is reached at a distorted lattice, i.e. $Q_{x}, Q_{y} \neq 0$. Without any quadratic coupling $(\mathrm{G}=0)$ the energy potential has the form

$$
V=\frac{1}{2} K\left(Q_{x}^{2}+Q_{y}^{2}\right)-F\left(Q_{x}^{2}+Q_{y}^{2}\right)^{\frac{1}{2}}
$$

This potential is minimal on radii

$$
\rho_{0}=\left(Q_{x}^{2}+Q_{y}^{2}\right)^{\frac{1}{2}}=F / K
$$

and is often referred to as Mexican hat potential due to its form. Compared to $Q_{x}=$ $Q_{y}=0$ the energy is lowered by $E_{J T}=F^{2} / 2 K$. The system can evolve fast between the nuclear configurations lying on this energy minimum circle. This situation is refereed to as dynamic Jahn-Teller effect. Including the quadratic coupling the energy potential takes the form [133]

$$
V=\frac{1}{2} K \rho^{2}-\rho\left[F^{2}+G^{2} \rho^{2}+2 F G \rho^{\frac{1}{2}} \cos (3 \phi)\right]^{\frac{1}{2}},
$$

where $\rho=\left(Q_{x}^{2}+Q_{y}^{2}\right)^{\frac{1}{2}}$ and $\phi=\arctan \left(Q_{y} / Q_{X}\right)$. There are now 3 minima in the energy potential surface lying on a radius of $\rho_{0}=F /(K-2 G)$ at angles $\phi=0,2 \pi / 3$ and $4 \pi / 3$ as shown in Fig. 2.8(a). The mean energy on the circle with radius $\rho_{0}$ is $E_{J T}=$ $F^{2} / 2(K-2 G)$ and the energy barrier between the minima is $\delta=4 E_{J T} G /(K+2 G)$. Between these minima tunneling might occur. If the barrier is much larger than the phonon energy, the system will be trapped in one of the minima and one speaks of the static Jahn-Teller effect. By first principles calculations Abtew et al. derived values for these parameters for the NV center [133]: $E_{J T}=25 \mathrm{meV}$ and $\delta=10 \mathrm{meV}$ with $F=-$ $0.74 \mathrm{eV} / \AA, G=1.76 \mathrm{eV} / \AA^{2}$ and $K=14.5 \mathrm{eV} / \AA^{2}$. The energy for e-type local vibration modes in the excited state is $71 \mathrm{meV}$, which is much larger than the barrier. Thus tunneling will occur frequently, and dynamics is more described by hindered internal rotation, which leads to a dynamic Jahn-Teller effect for the NV center.

The e vibrations can induce transitions between the $E_{x}$ and $E_{y}$ level through a two-phonon Raman process [136]. This reduces the lifetimes of either ${ }^{3} E$ excited state leading to a dephasing of the optical transition and thus a broadening of the optical linewidth. Fu et al. showed that the width's temperature dependence follows essentially 
(a)

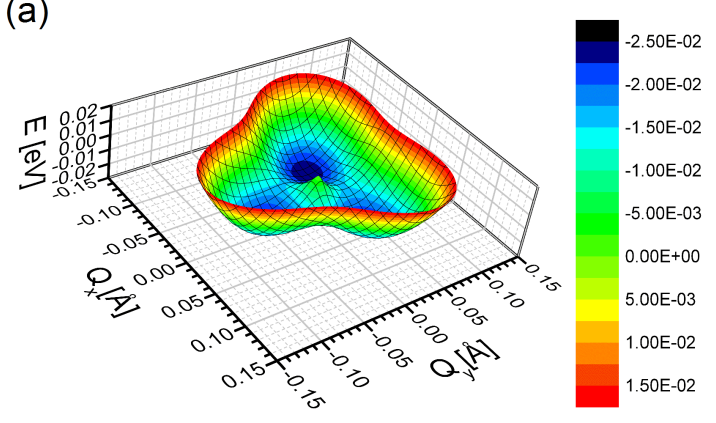

(b)

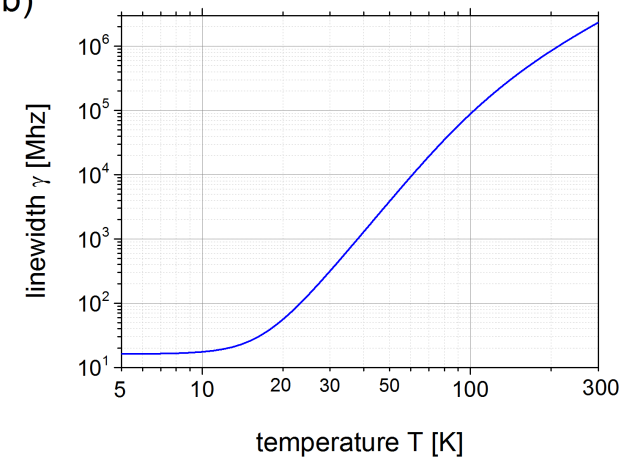

Figure 2.8: (a) Energy potential as given in equation 2.16 of the excited state as function of nuclear configuration $Q_{x}$ and $Q_{y}$ corresponding to normal coordinates for e vibrational local mode. The parameters used are the ones determined by Abtew et al. [133]. (b) Linewidth of the NV center as function of temperature broadened by Jahn-Teller interaction [133].

a $T^{5}$ law [136]. With the parameters of the Jahn-Teller coupling determined, Abtew et al. [133] were able to fully reproduce the experimentally measured linewidths as function of temperature theoretically. The width is given by:

$$
W(T)=\frac{8 \pi}{\hbar}\left(\frac{\Omega}{2 \pi^{2} \hbar^{3} v_{s}^{3}}\right)^{2} C^{4}\left(k_{B} T\right)^{5} I_{4}\left(\hbar \omega_{D} / k_{B} T\right),
$$

where $I_{4}(x)=\int_{0}^{x} d y y^{4} e^{y} /\left(e^{y}-1\right)^{2}$ is the Debye integral, $v_{s}=1.2 \times 10^{4} \mathrm{~m} / \mathrm{s}$ is speed of sound in diamond, $\Omega$ the volume of the unit cell in diamond. The coupling constant is $C=F \sqrt{1 /\left(2 m \omega_{0}^{2}\right)}$ with $F=-0.74 \mathrm{eV} / \AA$ at $\hbar \omega_{0}=71 \mathrm{meV}$ and $m$ being the mass of the carbon atom. To reproduce the measured values a cut-off energy of $\hbar \omega_{D}=50 \mathrm{meV}$ was needed [133]. The linewidth is then

$$
\gamma(T)=\gamma_{0}+2 W(T) /(2 \pi) \quad \text { with } \quad \gamma_{0}=16.2 \mathrm{MHz},
$$

which is plotted in Fig. 2.8(b) with the parameters given above.

Hizhnyakov et al. [137] reported a $T^{3}$ law. This is the only paper up to date which has reported this behavior. As origin for the $T^{3}$ dependence they proposed that the bonds and therefore the springs responsible for the transition soften. Because they performed only ensemble measurements, their results are only valid for high temperatures. As shown in Fig. 2.8(b) the slope of the linewidth changes for $T>100 \mathrm{~K}$ which can explain their results.

Earlier experiments by Davies [131] found a $T^{7}$ law according to a model by Maradudin [138] resulting from a linear electron-phonon coupling. These also were ensemble measurements. A significant $T^{7}$ contribution has not been found in later experiments.

In conclusion, according to today's knowledge the dynamic Jahn-Teller effect within the excited state of the NV center leads to a dephasing of the ZPL, that follows a $T^{5}$ law. Further contributions due to electron-phonon coupling terms are negligible. 
Additionally, solid state emitters often exhibit spectral diffusion. In contrast to the above described homogeneous broadening due to phonon scattering, this leads to an inhomogeneous broadening of the ZPL. The origin of spectral diffusion is associated to a change in the local static electric field the emitter is exposed to due to ionization of nearby defects by the excitation laser [139]. Via the Stark effect this leads to a shift of the ZPL frequency. For different NV centers there have been observed either linear or quadratic or both contributions to the Stark effect $[14,140]$. In diamond the main source for spectral diffusion is substitutional nitrogen, which has an optical ionization energy of $2.2 \mathrm{eV}[141,142]$. Note that the thermal activation energy is $1.7 \mathrm{eV}$ whereas the optical threshold is at $2.2 \mathrm{eV}$ [141]. This difference, which is a consequence of the Jahn-Teller effect, often leads to a confusion of the ionization energy of substitutional nitrogen. A single charge in a distance of $10 \mathrm{~nm}$ from the NV center can lead to a Stark shift of several hundreds $\mathrm{GHz}[14,143]$. When exciting off-resonantly at $532 \mathrm{~nm}$, spectral jumps occur on a fast timescale on the order of a few microseconds [143]. The recording of a spectrum of the fluorescence takes several seconds and thus the integrated form of the ZPL takes the form of a Gaussian. Also in typical resonant photo luminescence excitation (PLE) experiments, where a laser is scanned over the ZPL resonance and one detects the fluorescence on the sideband, spectral diffusion is observed: whereas an individual scan shows a narrow line the frequency center position changes when repeating the scan $[14,144-146]$. This mainly occurs when applying a re-pump laser, which is necessary, as resonant excitation leads to ionization of the NV center $[147,148]$. Recent experiments show when changing the laser wavelength of the re-pump from the conventional $532 \mathrm{~nm}$ to $575 \mathrm{~nm}$ (which is the ZPL wavelength of the $\mathrm{NV}^{0}$ ) spectral diffusion is suppressed [149]. This proves again that the main source for spectral diffusion is substitutional nitrogen, which at $575 \mathrm{~nm}$ is no longer ionized. It is worth noting that spectral diffusion strongly depends on the quality of the diamond ranging from a few hundred $\mathrm{GHz}$ in NDs [143] to smaller than $200 \mathrm{MHz}$ in ultra-pure diamond $[149,150]$ or good quality NDs [144]. In cases where the NV center is close to the surface, i.e. for shallow implanted NV centers or small NDs, surface defects acting as charge traps are a further source for spectral diffusion. In PLE measurements it is possible to dynamically stabilize the ZPL frequency via the Stark effect by applying a static external electric field [151].

\section{Charge state switching}

As mentioned above the NV center exists in different charge states. Because the main interest lies on the negatively charged $\mathrm{NV}^{-}$center, control over the charge state is desirable and many studies addressing this point have revealed rich physics concerning the process of charge state switching. The charge state of a NV center in the dark, i.e. under no optical excitation, is determined by its proximity to electron donors such as substitutional nitrogen [152]. For NV centers close to the surface, i.e. for shallow implanted NV centers or NV centers in NDs, additionally the surface termination, which changes the Fermi-level close to the surface, determines the charge state of the 
NV center $[150,153-156]$. The NV center is observed in its neutral charge state for a hydrogen terminated surface and in its negatively charged state for oxygen terminated surface. The physical explanation is given by Hauf et al. [155]: In the case of a oxygen terminated surface the Fermi level is mainly determined by the nitrogen concentration and the $\mathrm{NV}^{-}$level lies beneath the Fermi level and is thus stable. Hydrogen termination shifts the conduction and valence band upwards by $\approx 1 \mathrm{eV}$, such that the valence band is above the chemical potential on the surface, and a depletion of electrons from donors close to the surface occur. This leads to a strong band bending, and therefore close to the surface the $\mathrm{NV}^{-}$levels lie above the Fermi level and are ionized.

Optical illumination is a further source for change in the charge state. Under fs pulses the $\mathrm{NV}^{-}$center can be ionized permanently [157]. Also under continuous wave (cw) excitation photochromism has been observed [158]. Manson et al. [159] identified two mechanism for ionization depending on the excitation wavelength. If excitation is in the range from $500-640 \mathrm{~nm}$ the $\mathrm{NV}^{-}$is first excited and afterward ionized. The ionization is nearly linearly dependent on the excitation power. For wavelengths $<$ $490 \mathrm{~nm}$ the neutral substitutional nitrogen is efficiently ionized and thus their fraction is reduced. This leads to a change in the Fermi level such that the neutral charge state of the NV center becomes stable. By observing photochromism in a single NV center [23] Gaebel et al. showed that the charge state is continuously switched when the NV center is excited at $514 \mathrm{~nm}$. The fraction of NV centers being in the negatively charged state is for $450-610 \mathrm{~nm}$ excitation wavelength always $\leq 75 \%$ and maximal for excitation around 510-540 $\mathrm{nm}[148]$.

When the $\mathrm{NV}^{-}$center is excited resonantly, it is trapped in a dark state after a few cycles and a repump pulse at usually $532 \mathrm{~nm}$ is applied to regain fluorescence. Waldherr et al. [160] identified this dark state by single shot nuclear magnetic resonance (NMR) measurements as the neutral charge state. Beha et al. investigated the wavelength dependent charge transfer of the $\mathrm{NV}^{-}$[147] and identified the most efficient wavelength for recharging of the $\mathrm{NV}^{-}$is at $575 \mathrm{~nm}$. They thus proposed the following mechanism for recharging: The $\mathrm{NV}^{0}$ is first excited and in a second step a hole is transferred from the valence band to the ground state of the $\mathrm{NV}^{0}$. Therefore for resonant PLE measurements a repump at $575 \mathrm{~nm}$ is more efficient and moreover reduces spectral diffusion as less power is needed and substitutional nitrogen is no longer ionized [149].

\section{$2.2 \quad$ Fabry-Perot cavities}

This section describes the type of cavity used in this work, i.e. Fabry-Perot cavities, and their properties following mainly the textbook of Saleh and Teich [161]. A FabryPerot cavity is an optical resonator consisting of two opposing mirrors separated by a distance $l$ [see Fig. 2.9(a)]. In order for light to be kept confined between these two mirrors, the resonance condition has to be fulfilled: The distance between the mirrors has to be a multiple of half of the wavelength of the light such that a standing wave 
with nodes on the mirrors is formed:

$$
l=n \times \lambda / 2, \quad n \in \mathbb{N} .
$$

For a given cavity length the frequency difference between two neighboring modes is called the free spectral range (FSR):

$$
\nu_{\mathrm{FSR}}=\left|\nu_{n}-\nu_{n-1}\right|=\frac{c}{2 l} .
$$

We can also consider the resonator modes as traveling waves, which interfere with themselves after one round-trip. For constructive interference the acquired phase $\phi=$ $k 2 l=4 \pi \nu l / c$ needs to be a multiple of $2 \pi$. This is equivalent to the resonance condition as before (Eq. 2.19). If we also allow for losses such that the attenuation after one round-trip is given by $h=|r| e^{i \phi}$ we can write the total amplitude inside the resonator as series

$$
U=U_{0}+U_{1}+U_{2}+\ldots=U_{0}\left(1+h+h^{2}+\ldots\right) .
$$

The field intensity, which is the absolute value of the amplitude squared, is given by:

$$
I=|U|^{2}=\frac{\left|U_{0}\right|^{2}}{|1-| r\left|e^{i \phi}\right|^{2}}=\frac{I_{\max }}{1+(2 \mathcal{F} / \pi)^{2} \sin ^{2}\left(\pi \nu / \nu_{\mathrm{FSR}}\right)},
$$

where

$$
\mathcal{F}=\frac{\pi \sqrt{|r|}}{1-|r|} \quad \text { and } \quad I_{\max }=\frac{I_{0}}{(1-|r|)^{2}} .
$$

$I_{0}=\left|U_{0}\right|^{2}$ is the intensity of the initial wave and $\mathcal{F}$ is called the finesse. Figure 2.9(b) shows the intensity $I$ as function of the frequency $\nu$ for a cavity with a finesse of $\mathcal{F}=15$.

(a)

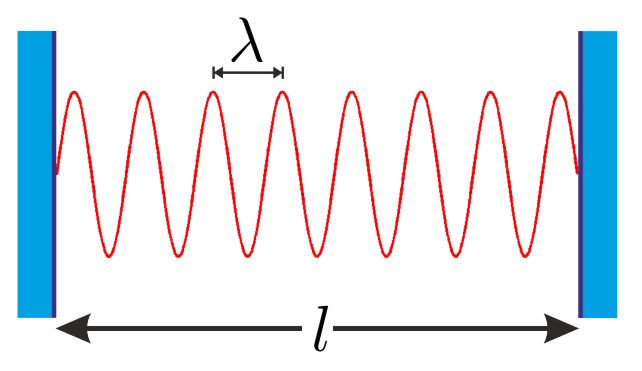

(b)

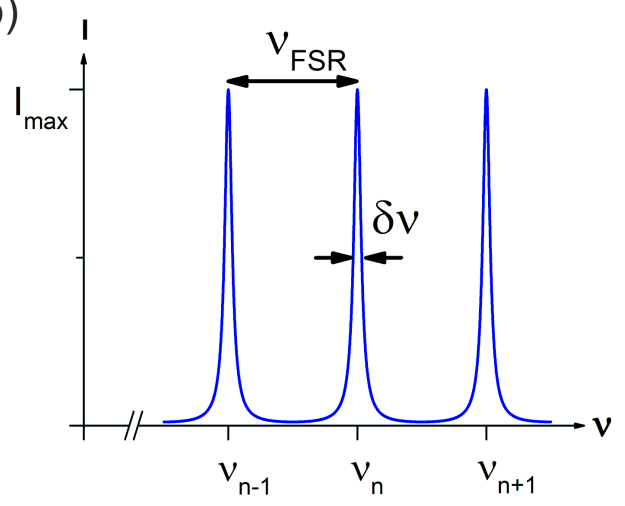

Figure 2.9: (a) Standing wave inside a Fabry-Perot cavity consisting of two opposing mirrors that are separated by a distance $l$. (b) Resonant modes of a cavity with finesse $\mathcal{F}=15$. The resonances are separated by the free spectral range $\nu_{\mathrm{FSR}}$. The width (FWHM) is given by $\delta \nu=\nu_{\mathrm{FSR}} / \mathcal{F}$. 
The spacing between two modes is still given by the FSR. The spectral width [full width at half maximum (FWHM)] of the modes is given by

$$
\delta \nu=\frac{\nu_{\mathrm{FSR}}}{\mathcal{F}} .
$$

If we assume two mirrors with intensity transmission coefficients $T_{1}, T_{2}$ and losses $L_{1}$, $L_{2}$, the amplitude attenuation factor is given by

$$
r=\sqrt{R_{1} R_{2}}=\sqrt{\left(1-T_{1}-L_{1}\right)\left(1-T_{2}-L_{2}\right)} .
$$

If $1-|r| \ll 1$ the finesse can be written as

$$
\mathcal{F} \approx \frac{\pi}{1-|r|} \approx \frac{2 \pi}{T_{1}+T_{2}+L_{1}+L_{2}}
$$

With an incident wave with intensity $I_{\text {inc }}$, the maximal intensity in resonance inside the resonator is given by $I_{\max }=\left(4 T_{1} I_{\text {inc }}\right) /\left(T_{1}+T_{2}+L_{1}+L_{2}\right)^{2}$, of which the fraction $T_{2}$ will be coupled out through the second mirror. Hence, the transmission of a wave in resonance is determined by

$$
T_{\text {resonance }}=\frac{4 T_{1} T_{2}}{\left(T_{1}+T_{2}+L_{1}+L_{2}\right)^{2}} .
$$

An effective method to determine the losses has been presented by Hood et al. [162] via measuring simultaneously the reflected and transmitted laser light when the cavity is in resonance with the laser frequency. The incoming light typically does not fully couple to the cavity mode due to a non-perfect spatial mode matching, i.e. only the fraction $\epsilon P_{\text {in }}$ from the input power $P_{\text {in }}$ couples to the cavity mode. Therefore, for the transmitted light

$$
T_{\text {resonance }}=\frac{P_{\text {trans }}}{\epsilon P_{\text {in }}}=\frac{4 T_{1} T_{2}}{\left(T_{1}+T_{2}+L_{1}+L_{2}\right)^{2}}=4 T_{1} T_{2}\left(\frac{\mathcal{F}}{2 \pi}\right)^{2},
$$

and for the reflected light

$$
\frac{P_{\text {refl }}-(1-\epsilon) P_{\text {in }}}{\epsilon P_{\text {in }}}=\left(L_{1}+L_{2}-T_{1}+T_{2}\right)^{2}\left(\frac{\mathcal{F}}{2 \pi}\right)^{2} .
$$

Combining these formulas one finds

$$
\frac{P_{\text {trans }}}{P_{\text {refl }}-P_{\text {in }}}=\frac{4 T_{1} T_{2}(\mathcal{F} /(2 \pi))^{2}}{\left(L_{1}+L_{2}+T_{1}-T_{2}\right)^{2}(\mathcal{F} /(2 \pi))^{2}-1} .
$$

In case of two identical mirrors $\left(T_{1}=T_{2} \equiv T\right.$ and $\left.L_{1}=L_{2} \equiv L\right)$ this allows, together with the knowledge of the finesse (cf. Eq. 2.26), to determine the transmission $T$ and losses $L$ of the coatings. 


\subsubsection{Gaussian beam}

Two plane mirrors need to be exactly parallel to keep a forth and backwards traveling ray laterally confined. This is technically challenging. Therefore, for stable resonators, which ensure the spatial confinement, concave mirrors are used. The modes of such a resonator are described by Gaussian beams. We follow their description given in the textbook of Saleh and Teich [161]. We consider the scalar wave equation

$$
\nabla^{2} U-\frac{1}{c^{2}} \frac{\partial^{2} U}{\partial t^{2}}=0
$$

for paraxial waves, i.e. we assume a slowly varying envelope approximation

$$
U(\vec{r})=A(\vec{r}) \exp (-i k z),
$$

with

$$
\frac{\partial A}{\partial z} \ll k A \text { and } \frac{\partial^{2} A}{\partial z^{2}} \ll k^{2} A .
$$

The complex envelope function $A(\vec{r})$ then satisfies the paraxial Helmoltz equation

$$
\nabla_{T} A-i 2 k \frac{\partial A}{\partial z}=0
$$

where $\nabla_{T}=\frac{\partial^{2}}{\partial x^{2}}+\frac{\partial^{2}}{\partial y^{2}}$. One of the solutions is of the form

$$
A(\vec{r})=\frac{A_{1}}{q(z)} \exp \left[-i k \frac{\rho^{2}}{2 q(z)}\right], \quad q(z)=z-\xi, \rho^{2}=x^{2}+y^{2}
$$

a paraboloidal wave centered about the point $z=\xi$. The case of a purely imaginary $\xi=-i z_{0}$ and defining $R(z)$ and $w(z)$ via

$$
\frac{1}{q(z)}=\frac{1}{z+i z_{0}} \equiv \frac{1}{R(z)}-i \frac{\lambda}{\pi w^{2}(z)}
$$

leads to the Gaussian beam

$$
U(\vec{r})=A_{0} \frac{w_{0}}{w(z)} \exp \left[-\frac{\rho^{2}}{w^{2}(z)}\right] \exp \left[-i k z-i k \frac{\rho^{2}}{2 R(z)}+i \zeta(z)\right],
$$

where

$$
\begin{aligned}
& w(z)=w_{0} \sqrt{1+\left(\frac{z}{z_{0}}\right)^{2}} \\
& R(z)=z\left[1+\left(\frac{z}{z_{0}}\right)^{2}\right] \\
& \zeta(z)=\tan ^{-1} \frac{z}{z_{0}}
\end{aligned}
$$




$$
w_{0}=\sqrt{\frac{\lambda z_{0}}{\pi}} .
$$

The transverse amplitude at any given position along the propagation axis $z$ has the form of a Gaussian. It's maximal on the axis and drops by a factor of $1 / e^{2}$ at a distance $w(z)$ from the axis, which is called the beam width. Note that the convention for laser beams is to specify as beam width the radius of the beam. The beam waist $w_{0}$ is the minimal beam width at the position $z=0$. The phase on the axis is $\phi(0, z)=k z-\zeta(z)$. Compared to a plane wave it is retarded by the so called Gouy phase $\zeta(z)$. The wave fronts are paraboloidal surfaces with radius of curvature $R(z)$. The parameter $z_{0}$ is known as Rayleigh range.

\section{Hermite-Gaussian beams}

The Gaussian Beam is not the only beam-like solution for the paraxial Helmholtz equation. The higher order mode are formed by a complex amplitude of the form

$$
\begin{aligned}
U_{l m}= & A_{l m} \frac{w_{0}}{w(z)} \mathcal{G}_{l}\left[\frac{\sqrt{2} x}{w(z)}\right] \mathcal{G}_{m}\left[\frac{\sqrt{2} y}{w(z)}\right] \\
& \times \exp \left[-i k z-i k \frac{x^{2}+y^{2}}{2 R(z)}+i(l+m+1) \zeta(z)\right],
\end{aligned}
$$

where $\mathcal{G}_{l}(u)$ is the Hermite-Gaussian function. Note that $\mathcal{G}_{0}(u)$ is simply a Gaussian function, and thus $U_{00}$ corresponds to the Gaussian beam as described above. Looking at the lateral intensity distribution, the parameters $l$ and $m$ give the number of nodes along the $x$ and $y$ direction. The curvature of the wave fronts is independent of $l$ and $m$ and the same $R(z)$ as before (Eq. 2.38b).

\subsubsection{Resonators with spherical mirrors}

Let's consider a Fabry-Perot resonator with spherical curved mirrors with radii of curvature $R_{1}$ and $R_{2}$ that are separated by a distance $l$. The $g$-parameters are defined as:

$$
g_{i}=1+l / R_{i}, \quad i=1,2 .
$$

The stability of such a resonator can be deduced from ray optics. In order for a ray, which is reflected between these two mirrors, to remain confined the following condition needs to be satisfied:

$$
0 \leq g_{1} g_{2} \leq 1
$$

At the boundary of this condition the resonator is said to be conditionally stable. The lower limit is the case of two plane mirrors, the upper limit the case of two curved mirrors separated by a distance $l=-\left(R_{1}+R_{2}\right)$. If the two mirrors are further apart the confinement condition is no longer satisfied and the resonator is said to be unstable. 
The resonant modes of such a resonator are naturally described by Hermite-Gaussian beams as they have paraboloidal curved wave fronts which can match the curvature of the mirrors as shown in Fig. 2.10. This is necessary for constructive interference of the

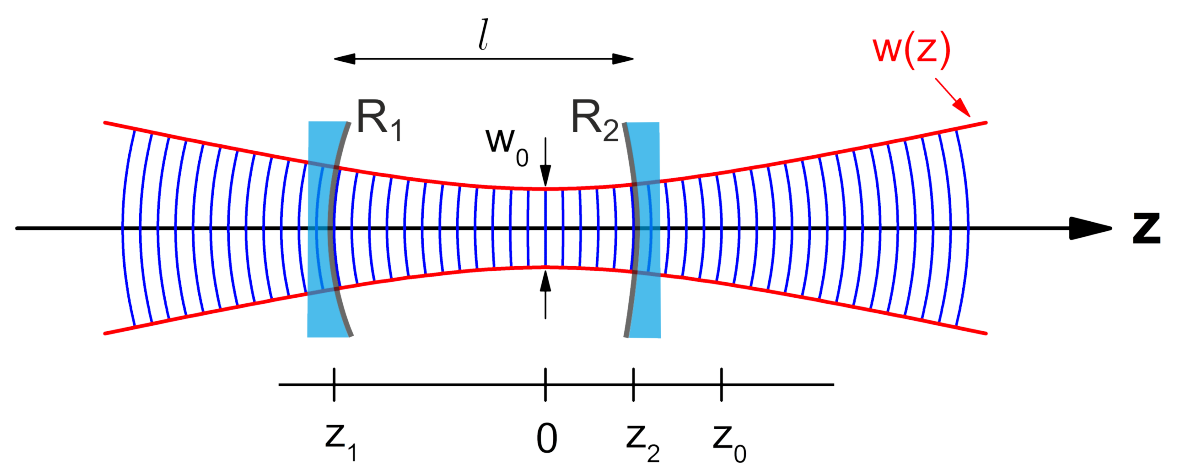

Figure 2.10: Matching the radii of curvature of the wave fronts of a Gaussian beam to the radii of curvature of the 2 cavity mirrors.

whole beam after one round-trip, which leads to the resonant modes of the resonator. The relative positions $z_{1}$ and $z_{2}$ of the mirrors with respect to the beam waist position at $z=0$ are given via the following matching conditions:

$$
\begin{aligned}
R_{1} & =R\left(z_{1}\right)=z_{1}\left[1+\left(\frac{z_{0}}{z_{1}}\right)^{2}\right] \\
-R_{2} & =R\left(z_{2}\right)=z_{2}\left[1+\left(\frac{z_{0}}{z_{2}}\right)^{2}\right] \\
l & =z_{2}-z_{1} .
\end{aligned}
$$

Note the difference in the conventions of the sign of radii of curvature for mirrors and beam. Whereas in case of the concave mirrors both radii of curvature are negative, for the beam the radius of curvature was defined to be positive for $z>0$ and negative otherwise. Solving equations 2.42 leads to:

$$
\begin{aligned}
z_{1} & =\frac{-l\left(R_{2}+d\right)}{R_{1}+R_{2}+2 l} \\
z_{2} & =z_{1}+l \\
z_{0}^{2} & =\frac{-l\left(R_{1}+d\right)\left(R_{2}+d\right)\left(R_{1}+R_{2}+d\right)}{\left(R_{2}+R_{1}+2 l\right)^{2}} .
\end{aligned}
$$

The resonance frequencies follow from the condition that the acquired round-trip phase needs to be a multiple of $2 \pi$. This yields

$$
\nu_{l, m, n}=n \times \nu_{\mathrm{FSR}}+(l+m+1) \frac{\Delta \zeta}{\pi} \nu_{\mathrm{FSR}},
$$


where $\Delta \zeta=\zeta_{z_{2}}-\zeta_{z_{1}}$. Modes with different $q$ but the same $l, m$ are known as longitudinal modes and are spaced in frequency by the FSR. The parameters $l, m$ specify the so called transverse mode. The Gaussian mode with $l=m=0$ is called the fundamental mode.

In the special case with one plane mirror and a second concave mirror with radius of curvature $R$ we find:

$$
\begin{aligned}
z_{1} & =0, \quad z_{2}=l \\
z_{0} & =l \sqrt{\frac{|R|}{l}-1} \\
w_{0}^{2} & =\sqrt{\lambda z_{0} / \pi}=\frac{\lambda l}{\pi} \sqrt{\frac{|R|}{l}-1 .}
\end{aligned}
$$

The mode volume $V_{\text {mod }}$ for such a cavity for the fundamental Gaussian mode is given by:

$$
V_{\text {mod }}=\frac{\int d^{3} r|\vec{E}(\vec{r})|^{2}}{\max |\vec{E}(\vec{r})|^{2}}=\frac{\pi}{4} w_{0}^{2} l=\frac{\lambda l^{2}}{4} \sqrt{\frac{|R|}{l}-1} .
$$

\subsection{Cavity quantum electrodynamics}

The realm of cavity QED is the description of the interaction between light and matter within a resonator where both (matter and light) have to be described quantummechanically. This chapter will present the basic concepts on the basis of a two level atom interacting with a single mode of an optical cavity. The discussion here is inspired by the very clear presentation of this topic in C. Russo's Ph.D. thesis [163]. First the interaction Hamiltonian will be introduced. Further, coupling to external reservoirs will lead to dissipation which has to be included. Depending on whether coherent evolution or the dissipation is dominant, different coupling regimes arise, and different physical phenomena such as Rabi oscillations or enhanced spontaneous emission can be observed.

\subsubsection{Jaynes-Cummings Hamiltonian}

We consider a two level atom with ground state $|0\rangle$ and excited state $|e\rangle$ with energies $\hbar \omega_{0}$ and $\hbar \omega_{e}$, respectively. The coupling between the 2-level atom and a single mode of a cavity is described by the Hamiltonian first introduced by Jaynes and Cummings [164]

$$
\hat{H}=\hbar \omega_{0} \hat{\sigma}_{00}+\hbar \omega_{e} \hat{\sigma}_{e e}+\hbar \omega_{c} \hat{a}^{\dagger} \hat{a}+\hbar\left(g \hat{\sigma}_{0 e}^{\dagger}-g^{\star} \hat{\sigma}_{0 e}\right)\left(\hat{a}-\hat{a}^{\dagger}\right),
$$

where $\hat{\sigma}_{i j}=|i\rangle\langle j|$ are the atomic population operators for $i=j$ and transition operators for $i \neq j$ and $\hat{a}^{\dagger}, \hat{a}$ are the creation and annihilation operator for a photon in the cavity mode with energy $\hbar \omega_{c}$. The coupling strength is given by the coupling constant $g$. It depends on the mode volume of the cavity $V_{\text {mod }}$ and the dipole moment $\mu$ of transition 
of the atom which can be related to the natural linewidth $\gamma_{0}$ :

$$
g_{0}=\sqrt{\frac{\mu^{2} \omega}{2 \hbar \epsilon_{0} V_{\text {mod }}}}=\sqrt{\frac{3 \lambda^{2} c \gamma_{0}}{4 \pi V_{\text {mod }}}} .
$$

The Heisenberg equations of motion for the case of vanishing coupling $(g \rightarrow 0)$ yield the time evolution for the decoupled operators as $\hat{a}(t)=\hat{a}(0) e^{-i \omega_{c} t}$ and $\hat{\sigma}_{0 e}(t)=$ $\hat{\sigma}_{0 e}(0) e^{-i\left(\omega_{e}-\omega_{0}\right) t}$. Near resonance $\left(\omega_{c} \approx \omega_{e}-\omega_{0}\right)$ the terms $\hat{a}^{\dagger} \hat{\sigma}_{0 e}^{\dagger}$ (creation of a photon and excitation of the two level atom) and $\hat{a} \hat{\sigma}_{0 e}$ (annihilation of a photon and de-excitation of the two level atom) violate energy conservation and oscillate fast $\left(\approx e^{ \pm 2 i \omega_{c} t}\right)$ and their effect on the dynamics compared to the slow terms $\left(\hat{a}^{\dagger} \hat{\sigma}_{0 e}, \hat{a} \hat{\sigma}_{0 e}^{\dagger}\right)$ averages out. Neglecting the fast terms gives the Jaynes-Cummings Hamiltonian in the rotating wave approximation:

$$
\hat{H}=\hbar \omega_{0} \hat{\sigma}_{00}+\hbar \omega_{e} \hat{\sigma}_{e e}+\hbar \omega_{c} \hat{a}^{\dagger} \hat{a}+\hbar g \hat{\sigma}_{0 e}^{\dagger} \hat{a}+\hbar g^{\star} \hat{\sigma}_{0 e} \hat{a}^{\dagger} .
$$

This can be rewritten as the sum of the non-interacting Hamiltonian $H_{0}$ for atom and cavity and the interaction Hamiltonian $H_{i}$ where

$$
\begin{aligned}
\hat{H}_{0} & =\hbar \omega_{0} \hat{\sigma}_{00}+\hbar \omega_{e} \hat{\sigma}_{e e}+\hbar \omega_{c} \hat{a}^{\dagger} \hat{a} \\
\hat{H}_{i} & =\hbar g \hat{\sigma}_{0 e}^{\dagger} \hat{a}+\hbar g^{\star} \hat{\sigma}_{0 e} \hat{a}^{\dagger} .
\end{aligned}
$$

The eigenstates of $H_{0}$ are tensor products of the atomic state $|0\rangle$ or $|e\rangle$ with a Fock state of the cavity $|n\rangle$ where $n$ is the number of photons in the cavity mode. Note that, if the atom is in resonance with the cavity, the states $|0, n\rangle$ and $|e, n-1\rangle$ are energetically degenerate states when considering only the non-interacting Hamiltonian $H_{0}$. The interaction Hamiltonian $H_{i}$ couples these two states to new eigenstates

$$
\begin{aligned}
& |n,+\rangle=\cos \theta_{n}|0, n\rangle-\sin \theta_{n}|e, n-1\rangle \\
& |n,-\rangle=\sin \theta_{n}|0, n\rangle+\cos \theta_{n}|e, n-1\rangle,
\end{aligned}
$$

with eigenvalues

$$
\lambda_{ \pm}^{(n)}=\hbar \omega_{c}\left(n-\frac{1}{2}\right) \pm \frac{\hbar}{2} \sqrt{\Delta^{2}+4 g^{2} n}, \quad \Delta=\left(\omega_{e}-\omega_{0}\right)-\omega_{c}
$$

where the mixing angle is given by

$$
\sin \theta_{n}=\frac{1}{\sqrt{2}}\left(1+\frac{\Delta}{\sqrt{\Delta^{2}+4 g^{2} n}}\right)^{1 / 2} .
$$

The two new levels $|n,+\rangle$ and $|n,-\rangle$ within the $\mathrm{n}$-th multiplicity are called the dressed states, which interact coherently at an effective Rabi frequency $\Omega^{(n)}=\sqrt{\Delta^{2}+4 g^{2} n}$. Each multiplicity is separated from the next one by $\hbar \omega_{c}$ the energy of the cavity photon. This model only holds if the splitting within each multiplicity is much smaller than the separation between the multiplicities i.e. $\hbar \Omega^{(n)} \ll \hbar \omega_{c}$. 


\subsubsection{Master equation}

In real physical systems there is no perfect isolation from the environment and thus dissipative processes have to be included. Describing an open quantum system coupled to an external reservoir is usually done within a master equation formalism. We will assume the Markov approximation: The reservoir is memoryless, i.e. the coupling to the reservoir does not depend on the past of the reservoir. This means all correlations between the system and the reservoir disappear much faster than the dynamics of the system. The master equation for the reduced density operator $\hat{\rho}$ then reads

$$
\frac{d \hat{\rho}}{d t}=\frac{i}{\hbar}[\hat{\rho}, \hat{H}]+\mathcal{L}_{\operatorname{diss}}(\hat{\rho}),
$$

where the dissipation is described by the superoperator $\mathcal{L}_{\text {diss }}$ in the Lindblad form in terms of the collapse operators $\hat{C}_{m}$ that govern the dissipative process:

$$
\mathcal{L}_{\text {diss }}=\sum_{m}\left(\hat{C}_{m} \hat{\rho} \hat{C}_{m}^{\dagger}-\frac{1}{2}\left(\hat{\rho} \hat{C}_{m}^{\dagger} \hat{C}_{m}+C_{m}^{\dagger} \hat{C}_{m} \rho\right)\right) .
$$

As dissipative processes we will have to consider de-excitation of the atom due to spontaneous emission of a photon into free space with rate $\gamma$ and loss of cavity photons, which are transmitted through or absorbed by one of the cavity mirrors with rate $\kappa$. To also take into account pure dephasing, which acts only on the off-diagonal elements of the reduced density matrix, we will have to include a further term in the master equation, which does not take the Lindblad form. For a two level atom it can be written as

$$
\mathcal{L}_{\text {dephasing }}=\frac{\gamma^{\star}}{4}\left(\hat{\sigma}_{z} \hat{\rho} \hat{\sigma}_{z}-\hat{\rho}\right), \quad \hat{\sigma}_{z}=\hat{\sigma}_{e e}-\hat{\sigma}_{00} .
$$

\subsubsection{Dissipation - coupling regimes}

Different coupling regimes arise depending on whether the coherent coupling governed by the coupling strength $g$ or the non-coherent processes governed by $\gamma$ and $\kappa$ dominate. There are two dimensionless characteristic parameters for the system [165]:

$$
\begin{aligned}
n_{0} & \equiv \frac{4 \gamma^{2}}{3 g^{2}} \\
N_{0} & \equiv \frac{2 \kappa \gamma}{g^{2}},
\end{aligned}
$$

where $n_{0}$ is the intracavity saturation photon number and $N_{0}$ is the critical atom number. The cooperativity parameter is defined as the inverse of the critical atom number $C \equiv 1 / N_{0}$. 


\section{Strong coupling regime}

The strong coupling regime is the limit where $g \gg \gamma, \kappa$. As in the lossless case the system is still described by multiplicities $|n, \pm\rangle$ with Rabi splitting $\hbar \Omega^{(n)}$, where $\Omega^{(n)}=$ $\sqrt{4 g^{2} n-(\gamma-\kappa)^{2}}$. In this regime the dynamic is characterized by coherent exchange between the excitation of the atom and the photon in the cavity within a multiplicity, which is faster than the irreversible processes, such that the emission of a photon is a reversible process. With the words of H.J. Kimble [166]: "The atom cavity system then has time to couple itself coherently and at least the possibility of a life of manifestly quantum dynamics before the grim reaper of dissipation enters." Occasional loss of the excitation leads to jumps to lower multiplicities until eventually the ground state $|g, 0\rangle$ is reached. In the strong coupling regime the critical atom number is smaller than one $\left(N_{0}<1\right)$, such that already a single atom within the cavity has a dramatic effect on the coupled system. Furthermore, the photon saturation number is also smaller than one $\left(n_{0}<1\right)$, which states that a single photon in the cavity field is sufficient for the atom to be affected by the cavity field. Strong coupling has first been observed for Rydberg atoms in microwave resonators [167] and later on also in the optical domain $[168,169]$.

\section{Weak coupling regime}

In the weak coupling regime the non-coherent losses dominate over the coherent coupling. In the bad cavity regime the limiting factor is cavity loss $(\kappa>\gamma \gg g)$, whereas in the bad emitter regime spontaneous emission into arbitrary modes is the fastest channel $(\gamma>\kappa \gg g)$.

\section{1.) Bad cavity regime}

Spontaneous emission of an emitter in free space is governed by its coupling to the vacuum field. The presence of the cavity changes the density of modes which can lead to enhancement or suppression of spontaneous emission. This effect is known as Purcell effect [67] and shall be described here following the presentation as given in the textbook of M. Fox [170]. According to Fermi's Golden rule the transition rate for spontaneous emission is given by

$$
W=\frac{2 \pi}{\hbar^{2}}\left|M_{12}\right|^{2} g(\omega),
$$

where $g(\omega)$ is the density of states and $M_{12}$ is the transition matrix element. In free space the density of states is given by:

$$
g(\omega)=\frac{\omega^{2} V_{0}}{\pi^{2} c^{3}}
$$

The dipole interaction gives the transition matrix element as $M_{12}=\vec{p} \cdot \vec{E}$. Spontaneous emission is the coupling to the vacuum electric field. Averaging over all possible orientations yields

$$
M_{12}^{2}=\frac{1}{3} \mu_{12}^{2} E_{\mathrm{vac}}^{2}=\frac{\mu_{12}^{2} \hbar \omega}{6 \epsilon_{0} V_{0}}
$$


where $\mu_{12}$ is the atomic dipole moment. Hence the spontaneous emission transition rate into free space is:

$$
W_{\text {free-space }}=\frac{\mu_{12}^{2} \omega^{3}}{3 \pi \epsilon_{0} \hbar c^{3}} .
$$

Within a cavity the density of states is changed. We assume a single mode with angular frequency $\omega_{c}$ and half width $\Delta \omega_{c}$ which is determined by the quality factor of the cavity via $Q=\omega_{c} / \Delta \omega_{c} \cdot g(\omega)$ is given by a normalized Lorentzian function:

$$
g(\omega)=\frac{2}{\pi \Delta \omega_{c}} \frac{\Delta \omega_{c}^{2}}{4\left(\omega-\omega_{c}\right)^{2}+\Delta \omega_{c}^{2}}
$$

At the frequency $\omega_{0}$ of the atom we have

$$
g\left(\omega_{0}\right)=\frac{2 Q}{\pi \omega_{0}} \frac{\Delta \omega_{c}^{2}}{4\left(\omega_{0}-\omega_{c}\right)^{2}+\Delta \omega_{c}^{2}} .
$$

In contrast to free-space the orientation of the electric field of the cavity mode is fixed and we don't have to average over arbitrary direction. With $\xi=(\vec{p} \cdot \vec{E}) /(|\vec{p}||\vec{E}|)$ the transition Matrix elements reads

$$
M_{12}=\xi^{2} \frac{\mu_{12}^{2} \hbar \omega}{2 \epsilon_{0} V_{0}}
$$

and hence the transition rate into the cavity mode is

$$
W_{\text {in-cavity }}=\frac{2 Q \mu_{12}}{\hbar \epsilon_{0} V_{0}} \xi^{2} \frac{\Delta \omega_{c}^{2}}{4\left(\omega_{0}-\omega_{c}\right)^{2}+\Delta \omega_{c}^{2}} .
$$

The Purcell factor is defined as the ratio between the emission rate in the cavity and the emission rate in free space:

$$
F_{p}=\frac{W_{\text {in-cavity }}}{W_{\text {free-space }}}=\frac{3 Q(\lambda / n)^{3}}{4 \pi^{2} V_{0}} \xi^{2} \frac{\Delta \omega_{c}^{2}}{4\left(\omega_{0}-\omega_{c}\right)^{2}+\Delta \omega_{c}^{2}} .
$$

In case of an ideally oriented dipole $(\xi=1)$ and the cavity being in resonance with the emitter $\left(\omega_{c}=\omega_{0}\right)$ we find the ideal Purcell factor:

$$
F_{p}=\frac{3 Q(\lambda / n)^{3}}{4 \pi^{2} V_{0}} .
$$

The faster spontaneous emission rate corresponds to a reduced lifetime of the excited state of the emitter in the cavity compared to the free space situation:

$$
\tau_{\text {in-cavity }}=\frac{\tau_{\text {free }- \text { space }}}{F_{p}} .
$$

The ratio of the emission into the cavity to the total emission is given by the $\beta$-factor defined as:

$$
\beta=\frac{W_{\text {in-cavity }}}{W_{\text {in-cavity }}+W_{\text {free-space }}}=\frac{F_{p}}{1+F_{p}} .
$$




\section{2.) Bad emitter regime}

In all previous discussions we have assumed an ideal emitter which has a Fourier-limited linewidth, i.e.

$$
\gamma=1 /(2 \tau)=\frac{\omega^{3} \mu^{2}}{6 \pi \epsilon_{0} \hbar c^{3}} .
$$

We can express the Purcell factor via the cooperativity by

$$
F_{p}=2 \times C=\frac{g^{2}}{\kappa \gamma} .
$$

For a Fourier-limited emitter this is equivalent to the expression above (cf Eq. 2.67) using

$$
g=\sqrt{\frac{\mu^{2} \omega}{2 \hbar \epsilon_{0} V}}=\sqrt{\frac{3 \lambda^{2} c \gamma}{4 \pi V}}
$$

and

$$
Q=\frac{\nu}{\delta \nu}=\frac{\pi c}{\kappa \lambda} .
$$

The NV center at room temperature, as discussed above, experiences large line broadening. The dominant effect for the ZPL is the Jahn-Teller effect leading, by a two-phonon Raman process, to a linewidth as given in Eq. 2.17. In a more general way we can describe this broadening by a pure dephasing term $\gamma^{\star}$, such that the linewidth of the emitter is now given by $\gamma+\gamma^{\star}$. If this total width is larger than the cavity decay rate $\gamma+\gamma^{\star}>\kappa$, we are in the bad emitter regime. Only the ratio $\kappa /\left(\gamma+\gamma^{\star}\right)$ lies within the cavity bandwidth and the modified Purcell-factor is:

$$
F_{p_{\text {bad-emitter }}}=\frac{g^{2}}{\kappa \gamma} \times \frac{\kappa}{\gamma+\gamma^{\star}}=\frac{g^{2}}{\left(\gamma+\gamma^{\star}\right) \gamma} .
$$

Most remarkably in this regime the coupling is independent of the cavity decay rate $\kappa$. In the next section this regime will be discussed more rigorously for the concrete case of a NV center coupled to a cavity.

\subsection{Single nitrogen-vacancy center in a microcavity}

\subsubsection{Conventions}

In this section the theoretical framework to describe the coupling of a single NV center to a microcavity will be developed, which is based on the work of Auffèves et al. [171-173]. They use a different convention for $\kappa$ and $\gamma$, i.e. these rates describe the population decay, which is twice as fast as the decay of the coherences. In the atom physics community, which convention has been used in the previous section, this rates usually describe the decay of the coherences.

From now on $\boldsymbol{\kappa}$ and $\gamma$ will describe the population decay in order to be consistent with Auffèves and our own publication [174]. To provide a clear distinction bold symbols are used. 


\subsubsection{Model}

\section{Overview}

We expand a master equation model [172] for solid-state emitter-cavity coupling. For the NV center we assume, in analogy to [13], a simplified level scheme as depicted in Fig. 2.11(b), consisting of one excited state $(|e\rangle)$ and $n+1$ vibronic ground states $\left(\left|g_{0}\right\rangle, \ldots,\left|g_{n}\right\rangle\right)$. The spontaneous decay rate on the ZPL $\left(|e\rangle \rightarrow\left|g_{0}\right\rangle\right)$ is denoted by $\gamma_{0}$, on the PSB $\left(|e\rangle \rightarrow\left|g_{i}\right\rangle\right)$ by $\gamma_{i}$. At room temperature there are two dominant line broadening mechanisms: first, all transitions are equally subject to phonon scattering, which can be described by a pure dephasing rate $\gamma^{\star}$; second, the PSB experience an additional radiative broadening $\gamma_{i, i-1}$ due to the short lifetime of the vibrational levels $\left|g_{i}\right\rangle$. This level scheme represents a simplified description of the NV center PSB spectrum but suffices to adequately account for the contributions of the individual transitions to the cavity coupling. Discrete vibrational levels for the NV center have been calculated [132-134] and measured [135]. Thus our approach is well justified.

The model makes several additional simplifications: Only the optical transition between excited state and ground state is considered. [By measuring the photon statistics the inter-system crossing to the singlet states and charge state switching can be separated and the strength of the optical transition be determined separately (see Sect. 3.2)]. In the experiment cw off-resonant excitation at $532 \mathrm{~nm}$ is used and thereby the spin is continuously polarized into the $m_{s}=0$ spin state. Thus we don't have to consider different spin sublevels and can omit the fact of different excited state lifetimes for the different spin sublevels. The PSB are accounted for by a Huang-Rhys model. But to precisely reproduce the spectrum we do not assume a single vibronic mode with a harmonic potential leading to equidistant vibronic sublevels, instead determine the energies of the vibronic sublevels directly by fitting the spectrum as shown in Fig 2.11(a). Note that this fit is not unique because of strongly overlapping vibrational bands and thus it is only one of the possible solutions to reproduce the spectrum. In fact, there exist more not resolved levels than determined by the fit [132-135]. The number of vibronic levels considered here is the minimum number to adequately describe the full NV spectrum. The obtained parameters from the fit of spectra of several different single NV centers are listed and discussed in Sect. 3.3. As mentioned before the pure dephasing takes into account the above discussed dynamic Jahn-Teller effect, which is the dominant line-broadening mechanism for the ZPL.

The coupling of the optical transition to the cavity mode is described by a JaynesCummings Hamiltonian:

$$
\begin{aligned}
\hat{\mathcal{H}}^{J C}= & \sum_{i=0}^{n} \hbar \omega_{i} \hat{\sigma}_{i, i}+\hbar \omega_{e} \hat{\sigma}_{e, e}+\hbar \omega_{c} \hat{a}^{\dagger} \hat{a} \\
& +\hbar \sum_{i=0}^{n} g_{i}\left(\hat{a}^{\dagger} \hat{\sigma}_{i, e}+\hat{\sigma}_{i, e}^{\dagger} \hat{a}\right),
\end{aligned}
$$

where $\hbar \omega_{i}, i=0,1, \ldots n$ are the energies of the vibrational levels in the ground state, 
(a)

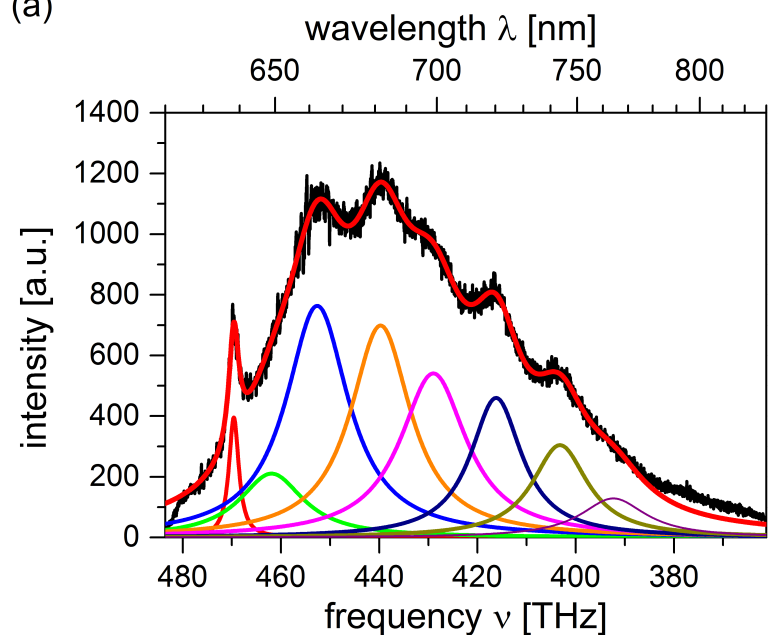

(b)

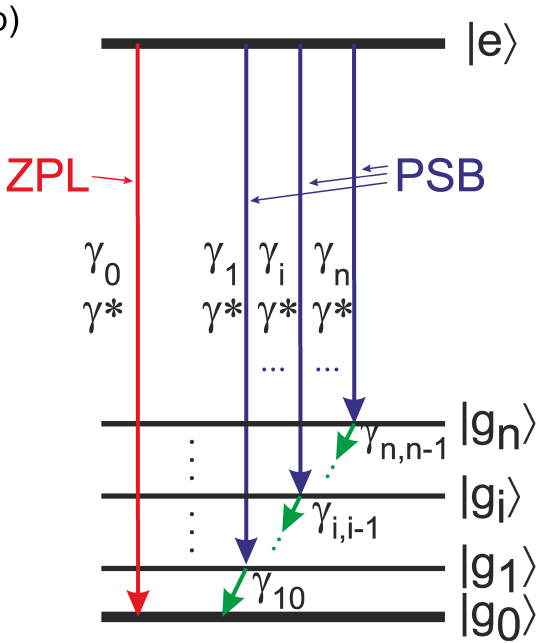

Figure 2.11: (a) Room-temperature emission spectrum of a single NV center. The spectrum has been fitted with 8 Lorentzian lines to deduce the model parameters. (b) Simplified level scheme as assumed in the model described above with $\mathrm{n}$ vibronic ground states $\left|g_{i}\right\rangle$ and one excited state $|e\rangle$. Broadening of the lines is due to spontaneous emission $\left(\gamma_{i}\right)$, pure dephasing $\left(\gamma^{\star}\right)$ and emission of phonons $\left(\gamma_{i, i-1}\right)$.

$\hbar \omega_{e}$ is the excited state energy, $\omega_{c}$ is the cavity resonance frequency, $g_{i}$ are the cavity coupling rates, $\hat{a}\left(\hat{a}^{\dagger}\right)$ are cavity photon annihilation(creation) operators and $\hat{\sigma}_{n, m}$ are transition $(n \neq m)$ or population $(n=m)$ operators. Assuming the Markov approximation the master equation for the coupled system is:

$$
\dot{\rho}=\frac{i}{\hbar}\left[\rho, \hat{\mathcal{H}}^{J C}\right]+\mathcal{L}_{\text {damp }}^{\text {cav }}+\mathcal{L}_{\text {damp }}^{\text {at }}+\mathcal{L}_{\mathrm{GS}-\text { Relax }}^{\text {at }}+\mathcal{L}_{\text {deph }}^{\text {at }} .
$$

The first term describes the coherent Jaynes-Cummings interaction. The further terms take into account cavity loss with a rate $\boldsymbol{\kappa}$

$$
\mathcal{L}_{\text {damp }}^{\text {cav }}=\kappa \mathcal{L}[\hat{a}, \rho],
$$

loss of polarization

$$
\mathcal{L}_{\text {damp }}^{\text {at }}=\sum_{i=0}^{n} \gamma_{i} \mathcal{L}\left[\hat{\sigma}_{i e}, \rho\right]
$$

and broadening effects as discussed above: these are broadening effects due to fast relaxation within the vibrational ground states

$$
\mathcal{L}_{\mathrm{GS}-\text { Relax }}^{\text {at }}=\sum_{i=0}^{n} \gamma_{i+1, i} \mathcal{L}\left[\hat{\sigma}_{i, i+1}, \rho\right]
$$


and broadening due to pure dephasing

$$
\mathcal{L}_{\text {deph }}^{\text {at }}=-\frac{\gamma^{\star}}{4}\left(\hat{\tilde{\sigma}}_{z} \rho \hat{\tilde{\sigma}}_{z}-\rho\right), \quad \hat{\tilde{\sigma}}_{z} \equiv \hat{\sigma}_{e e}-\sum_{i=0}^{n} \hat{\sigma}_{i i} .
$$

$\mathcal{L}[\hat{O}, \rho]$ denotes the standard Lindblad form defined as:

$$
\mathcal{L}[\hat{O}, \rho] \equiv \hat{O} \rho \hat{O}^{\dagger}-\frac{1}{2}\left(\hat{O}^{\dagger} \hat{O} \rho+\rho \hat{O}^{\dagger} \hat{O}\right)
$$

The master equation can be used to calculate the full time evolution of populations and coherences. At room temperature, however, line broadening dominates all other rates and coherences can be adiabatically eliminated leading to a rate equation system

$$
\begin{aligned}
\frac{d}{d t}\left\langle\hat{a}^{\dagger} \hat{a}\right\rangle & =-\left(\boldsymbol{\kappa}+R_{0}\right)\left\langle\hat{a}^{\dagger} \hat{a}\right\rangle+\sum_{i=0}^{n} R_{i}\left\langle\hat{\sigma}_{e e}\right\rangle \\
\frac{d}{d t}\left\langle\hat{\sigma}_{e e}\right\rangle & =-\left(\boldsymbol{\gamma}+\sum_{i=0}^{n} R_{i}\right)\left\langle\hat{\sigma}_{e e}\right\rangle+R_{0}\left\langle\hat{a}^{\dagger} \hat{a}\right\rangle .
\end{aligned}
$$

An explicit derivation is given later on. In analogy to [172] the rate equations' dynamics are governed by effective coupling rates between the emitter's transition and the cavity mode:

$$
\begin{aligned}
R_{0} & =\frac{4 g_{0}^{2}}{\kappa+\gamma+\gamma^{\star}} \frac{1}{1+\left(\frac{2 \delta_{0}}{\kappa+\gamma+\gamma^{\star}}\right)^{2}} \\
R_{i} & =\frac{4 g_{i}^{2}}{\kappa+\gamma+\gamma_{i, i-1}+\gamma^{\star}} \frac{1}{1+\left(\frac{2 \delta_{i}}{\kappa+\gamma+\gamma_{i, i-1}+\gamma^{\star}}\right)^{2}},
\end{aligned}
$$

where $i=1 \ldots n, \gamma=\gamma_{0}+\gamma_{1}+\ldots+\gamma_{n}$ and $\delta_{i}=\left(\omega_{e}-\omega_{i}\right)-\omega_{c}$. For fast cavity decay $\kappa>>\gamma, R_{i}$ we determine the emission efficiency $P_{i}$ of each transition as ratio of the emission coupled into the cavity to the total NV emission:

$$
P_{i}=\frac{R_{i}}{\gamma+\sum_{j=0}^{n} R_{j}}, \quad i=0, \ldots, n
$$

If the cavity is tuned to any arbitrary wavelength $\lambda$ within the NV emission spectrum, the total emission into the cavity mode is the sum of contributions from ZPL and all PSBs:

$$
P_{\mathrm{tot}}(\lambda)=\sum_{i=0}^{n} P_{i}(\lambda)=\frac{\sum_{i=0}^{n} R_{i}(\lambda)}{\gamma+\sum_{j=0}^{n} R_{j}(\lambda)} .
$$




\section{Experiments with quantum dots}

This theoretical framework has not been explicitly considered for coupling of an NV center to a microcavity in previous work. However, it has been investigated theoretically $[171-173,175-178]$ and experimentally $[179-188]$ for cavity-coupled quantum dots (QD). One of the key features of these experiments is the observation of emission into the cavity even for a far-detuned QD (detuning greater than the linewidths of the QD and cavity). This fact, which seemed at first glance to violate energy conservation, came as a surprise in the early experiments. Dephasing assisted channeling into the cavity line due to coupling with optical phonons was postulated theoretically as source for the observed phenomena $[171,177,178]$ and verified later on experimentally [184-186]. An classical picture was given by Naesby et al. [177]. In the far detuned case one can think of the system as driven damped harmonic oscillator, where the cavity is the oscillator and the emitter the drive. With a continuously steady drive, the oscillation at the resonators frequency diminishes and only oscillation at the drive frequency will survive. Dephasing can now be seen as instantaneous jumps of the drive position occurring on a fast timescale, such that the transient oscillation at the resonators frequency is continuously restarted. Thus, oscillation at both frequencies will persist.

\section{Explicit derivation of the rate model from the master equation}

We model the NV center with $\mathrm{n}+1$ vibronic ground states $\left|g_{i}\right\rangle$ and one excited state $|e\rangle$. To increase the readability of the derivation we here restrict ourselves to three vibrational ground state levels. The generalization to $\mathrm{n}$ ground state levels, however, is straightforward.

(a)

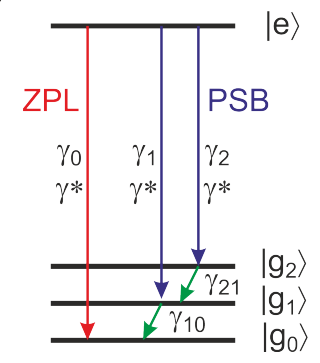

(b)

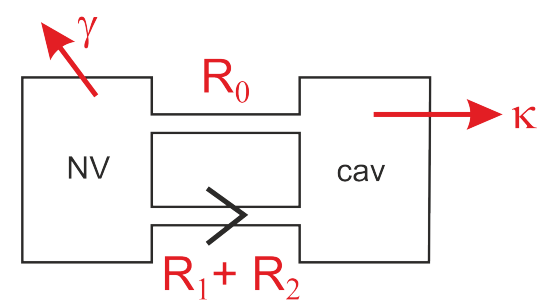

Figure 2.12: (a) Model of the NV center with 3 vibronic ground states $\left|g_{0}\right\rangle,\left|g_{1}\right\rangle,\left|g_{2}\right\rangle$ and one excited state $|e\rangle$. All optical transitions have spontaneous emission rates $\gamma_{i}$ and pure dephasing rates of $\boldsymbol{\gamma}^{*}$. (b) Simplified model (cf. [172]) for NV center in the cavity consisting of two boxes (NV center and cavity) coupled with the rates $R_{0}, R_{1}$ and $R_{2}$ where the last two rates are only one-directional. The loss rate from the atom (cavity) box is $\gamma=\gamma_{0}+\gamma_{1}+\gamma_{2}(\boldsymbol{\kappa})$, respectively.

The model is depicted in Fig. 2.12(a). The NV center is simplified by three vibronic ground states $\left(\left|g_{0}\right\rangle,\left|g_{1}\right\rangle,\left|g_{2}\right\rangle\right)$ and one excited state $(|e\rangle)$. The spontaneous decay on the ZPL is denoted by $\gamma_{0}$ and on the PSB by $\gamma_{1}$ and $\gamma_{2}$. Each optical transition has a pure 
dephasing rate of $\gamma^{*}$. The higher vibronic ground states decay rapidly to the ground state by rates $\gamma_{21}$ and $\gamma_{10}$. All three optical transitions are coupled to the cavity by coupling rates $g_{0}, g_{1}$ and $g_{2}$. The cavity has a decay rate of $\boldsymbol{\kappa}$. To remember, we here use the convention that all decay rates $\left(\gamma_{i}, \gamma_{i j}\right.$ and $\left.\boldsymbol{\kappa}\right)$ describe loss of population, whereas decay of the coherences is at half of these rates. The Jaynes Cummings Hamiltonian describing the system is given by:

$$
\begin{aligned}
\hat{\mathcal{H}}^{J C}= & \hbar \omega_{0} \hat{\sigma}_{00}+\hbar \omega_{1} \hat{\sigma}_{11}+\hbar \omega_{2} \hat{\sigma}_{22}+\hbar \omega_{e} \hat{\sigma}_{e e} \\
& +\hbar \omega_{c} \hat{a}^{\dagger} \hat{a} \\
& +\hbar\left(g_{0}\left(\hat{a}^{\dagger} \hat{\sigma}_{0 e}+\hat{\sigma}_{0 e}^{\dagger} \hat{a}\right)+g_{1}\left(\hat{a}^{\dagger} \hat{\sigma}_{1 e}+\hat{\sigma}_{1 e}^{\dagger} \hat{a}\right),+g_{2}\left(\hat{a}^{\dagger} \hat{\sigma}_{2 e}+\hat{\sigma}_{2 e}^{\dagger} \hat{a}\right)\right)
\end{aligned}
$$

where $\hbar \omega_{i}(i=1,2,3, e)$ are the energies of the levels of the NV center, $\omega_{c}$ is the frequency of the cavity, $\hat{a}, \hat{a}^{\dagger}$ are the annihilation and creation operator for a photon in the cavity and $\hat{\sigma}_{i j}(i, j=0,1,2, e)$ are the transition operators from level $\mathrm{j}$ to $\mathrm{i}$ for $i \neq j$ and the population operators for $i=j$.

Assuming the Markov approximation the master equation for the system is:

$$
\dot{\rho}=\frac{i}{\hbar}\left[\rho, \hat{\mathcal{H}}^{J C}\right]+\mathcal{L}_{\text {damp }}^{\text {cav }}+\mathcal{L}_{\text {damp }}^{\text {at }}+\mathcal{L}_{\mathrm{GS}-\text { Relax }}^{\text {at }}+\mathcal{L}_{\text {deph }}^{\text {at }} .
$$

We take into account:

cavity loss:

$$
\mathcal{L}_{\text {damp }}^{\text {cav }}=\boldsymbol{\kappa} \mathcal{L}[\hat{a}, \rho]
$$

loss of polarization:

$$
\mathcal{L}_{\text {damp }}^{\text {at }}=\gamma_{0} \mathcal{L}\left[\hat{\sigma}_{0 e}, \rho\right]+\gamma_{1} \mathcal{L}\left[\hat{\sigma}_{1 e}, \rho\right]+\gamma_{2} \mathcal{L}\left[\hat{\sigma}_{2 e}, \rho\right]
$$

lifetime broadening of the PSB:

$$
\mathcal{L}_{\text {GS-Relax }}^{\text {at }}=\gamma_{10} \mathcal{L}\left[\hat{\sigma}_{01}, \rho\right]+\gamma_{21} \mathcal{L}\left[\hat{\sigma}_{12}, \rho\right]
$$

and broadening due to pure dephasing:

$$
\mathcal{L}_{\text {deph }}^{\text {at }}=-\frac{\gamma^{\star}}{4}\left(\hat{\tilde{\sigma}}_{z} \rho \hat{\tilde{\sigma}}_{z}-\rho\right)=-\frac{\gamma^{\star}}{2}\left(\begin{array}{cccc}
0 & \rho_{12} & \rho_{13} & \rho_{14} \\
\rho_{21} & & & \\
\rho_{31} & & 0 & \\
\rho_{41} & & &
\end{array}\right),
$$

where:

$$
\begin{aligned}
& \hat{\tilde{\sigma}}_{z} \equiv \hat{\sigma}_{e e}-\hat{\sigma}_{00}-\hat{\sigma}_{11}-\hat{\sigma}_{22} \\
& \mathcal{L}[\hat{O}, \rho] \equiv \hat{O} \rho \hat{O}^{\dagger}-\frac{1}{2}\left(\hat{O}^{\dagger} \hat{O} \rho+\rho \hat{O}^{\dagger} \hat{O}\right) .
\end{aligned}
$$


We calculate the time evolution of the populations of the atom levels and the photon number within the cavity:

$$
\begin{aligned}
\frac{d}{d t}\left\langle\hat{\sigma}_{e e}\right\rangle= & +g_{0}\left(i\left\langle\hat{a}^{\dagger} \hat{\sigma}_{0 e}\right\rangle-i\left\langle\hat{\sigma}_{0 e}^{\dagger} \hat{a}\right\rangle\right)+g_{1}\left(i\left\langle\hat{a}^{\dagger} \hat{\sigma}_{1 e}\right\rangle-i\left\langle\hat{\sigma}_{1 e}^{\dagger} \hat{a}\right\rangle\right) \\
& +g_{2}\left(i\left\langle\hat{a}^{\dagger} \hat{\sigma}_{2 e}\right\rangle-i\left\langle\hat{\sigma}_{2 e}^{\dagger} \hat{a}\right\rangle\right)-\left(\gamma_{0}+\gamma_{1}+\gamma_{2}\right)\left\langle\hat{\sigma}_{e e}\right\rangle \\
\frac{d}{d t}\left\langle\hat{\sigma}_{00}\right\rangle= & -g_{0}\left(i\left\langle\hat{a}^{\dagger} \hat{\sigma}_{0 e}\right\rangle-i\left\langle\hat{\sigma}_{0 e}^{\dagger} \hat{a}\right\rangle\right)+\gamma_{0}\left\langle\hat{\sigma}_{e e}\right\rangle+\gamma_{10}\left\langle\hat{\sigma}_{11}\right\rangle \\
\frac{d}{d t}\left\langle\hat{\sigma}_{11}\right\rangle= & -g_{1}\left(i\left\langle\hat{a}^{\dagger} \hat{\sigma}_{1 e}\right\rangle-i\left\langle\hat{\sigma}_{1 e}^{\dagger} \hat{a}\right\rangle\right)+\gamma_{1}\left\langle\hat{\sigma}_{e e}\right\rangle-\gamma_{10}\left\langle\hat{\sigma}_{11}\right\rangle+\gamma_{21}\left\langle\hat{\sigma}_{22}\right\rangle \\
\frac{d}{d t}\left\langle\hat{\sigma}_{22}\right\rangle= & -g_{2}\left(i\left\langle\hat{a}^{\dagger} \hat{\sigma}_{2 e}\right\rangle-i\left\langle\hat{\sigma}_{2 e}^{\dagger} \hat{a}\right\rangle\right)+\gamma_{2}\left\langle\hat{\sigma}_{e e}\right\rangle-\gamma_{21}\left\langle\hat{\sigma}_{22}\right\rangle \\
\frac{d}{d t}\left\langle\hat{a}^{\dagger} \hat{a}\right\rangle= & -g_{0}\left(i\left\langle\hat{a}^{\dagger} \hat{\sigma}_{0 e}\right\rangle-i\left\langle\hat{\sigma}_{0 e}^{\dagger} \hat{a}\right\rangle\right)-g_{1}\left(i\left\langle\hat{a}^{\dagger} \hat{\sigma}_{1 e}\right\rangle-i\left\langle\hat{\sigma}_{1 e}^{\dagger} \hat{a}\right\rangle\right) \\
& -g_{2}\left(i\left\langle\hat{a}^{\dagger} \hat{\sigma}_{2 e}\right\rangle-i\left\langle\hat{\sigma}_{2 e}^{\dagger} \hat{a}\right\rangle\right)-\kappa\left\langle\hat{a}^{\dagger} \hat{a}\right\rangle,
\end{aligned}
$$

and of the coherences:

$$
\begin{aligned}
\frac{d}{d t}\left\langle\hat{\sigma}_{0 e}^{\dagger} \hat{a}\right\rangle= & i \underbrace{\left(\left(\omega_{e}-\omega_{0}\right)-\omega_{c}\right)}_{\delta_{0}}\left\langle\hat{\sigma}_{0 e}^{\dagger} \hat{a}\right\rangle-\frac{\boldsymbol{\kappa}+\gamma_{0}+\gamma_{1}+\gamma_{2}+\gamma^{\star}}{2}\left\langle\hat{\sigma}_{0 e}^{\dagger} \hat{a}\right\rangle \\
& -i g_{0}\left(\left\langle\hat{\sigma}_{e e}\right\rangle-\left\langle\hat{a}^{\dagger} \hat{a}\left(\hat{\sigma}_{00}-\hat{\sigma}_{e e}\right)\right\rangle\right)+i g_{1}\left\langle\hat{\sigma}_{01}^{\dagger} \hat{a}^{\dagger} \hat{a}\right\rangle+i g_{2}\left\langle\hat{\sigma}_{02}^{\dagger} \hat{a}^{\dagger} \hat{a}\right\rangle \\
\frac{d}{d t}\left\langle\hat{\sigma}_{1 e}^{\dagger} \hat{a}\right\rangle= & i \underbrace{\left(\left(\omega_{e}-\omega_{1}\right)-\omega_{c}\right)}_{\delta_{1}}\left\langle\hat{\sigma}_{1 e}^{\dagger} \hat{a}\right\rangle-\frac{\boldsymbol{\kappa}+\gamma_{0}+\gamma_{1}+\gamma_{2}+\gamma_{10}+\gamma^{\star}}{2}\left\langle\hat{\sigma}_{1 e}^{\dagger} \hat{a}\right\rangle \\
& -i g_{1}\left(\left\langle\hat{\sigma}_{e e}\right\rangle-\left\langle\hat{a}^{\dagger} \hat{a}\left(\hat{\sigma}_{11}-\hat{\sigma}_{e e}\right)\right\rangle\right)+i g_{0}\left\langle\hat{a}^{\dagger} \hat{a} \hat{\sigma}_{01}\right\rangle+i g_{2}\left\langle\hat{\sigma}_{12}^{\dagger} \hat{a}^{\dagger} \hat{a}\right\rangle \\
\frac{d}{d t}\left\langle\hat{\sigma}_{2 e}^{\dagger} \hat{a}\right\rangle= & i \underbrace{\left(\left(\omega_{e}-\omega_{2}\right)-\omega_{c}\right)}_{\delta_{2}}\left\langle\hat{\sigma}_{2 e}^{\dagger} \hat{a}\right\rangle-\frac{\boldsymbol{\kappa}+\gamma_{0}+\gamma_{1}+\gamma_{2}+\gamma_{21}+\gamma^{\star}}{2}\left\langle\hat{\sigma}_{2 e}^{\dagger} \hat{a}\right\rangle \\
& -i g_{2}\left(\left\langle\hat{\sigma}_{e e}\right\rangle-\left\langle\hat{a}^{\dagger} \hat{a}\left(\hat{\sigma}_{22}-\hat{\sigma}_{e e}\right)\right\rangle\right)+i g_{0}\left\langle\hat{a}^{\dagger} \hat{a} \hat{\sigma}_{02}\right\rangle+i g_{1}\left\langle\hat{a}^{\dagger} \hat{a} \hat{\sigma}_{12}\right\rangle,
\end{aligned}
$$

and furthermore:

$$
\begin{aligned}
\frac{d}{d t}\left\langle\hat{\sigma}_{01}^{\dagger} \hat{a}^{\dagger} \hat{a}\right\rangle= & i\left(\omega_{1}-\omega_{0}\right)\left\langle\hat{\sigma}_{01}^{\dagger} \hat{a}^{\dagger} \hat{a}\right\rangle-i g_{0}\left\langle\hat{a}^{\dagger} \hat{a} \hat{a}^{\dagger} \hat{\sigma}_{1 e}\right\rangle+i g_{1}\left\langle\hat{\sigma}_{0 e}^{\dagger} \hat{a} \hat{a}^{\dagger} \hat{a}\right\rangle \\
& -\left(\boldsymbol{\kappa}+\frac{1}{2} \gamma_{10}\right)\left\langle\hat{\sigma}_{01}^{\dagger} \hat{a}^{\dagger} \hat{a}\right\rangle \\
\frac{d}{d t}\left\langle\hat{\sigma}_{02}^{\dagger} \hat{a}^{\dagger} \hat{a}\right\rangle= & i\left(\omega_{2}-\omega_{0}\right)\left\langle\hat{\sigma}_{02}^{\dagger} \hat{a}^{\dagger} \hat{a}\right\rangle-i g_{0}\left\langle\hat{a}^{\dagger} \hat{a} \hat{a}^{\dagger} \hat{\sigma}_{2 e}\right\rangle+i g_{2}\left\langle\hat{\sigma}_{0 e}^{\dagger} \hat{a} \hat{a}^{\dagger} \hat{a}\right\rangle \\
& -\left(\boldsymbol{\kappa}+\frac{1}{2} \gamma_{21}\right)\left\langle\hat{\sigma}_{02}^{\dagger} \hat{a}^{\dagger} \hat{a}\right\rangle
\end{aligned}
$$




$$
\begin{aligned}
\frac{d}{d t}\left\langle\hat{\sigma}_{12}^{\dagger} \hat{a}^{\dagger} \hat{a}\right\rangle= & i\left(\omega_{2}-\omega_{1}\right)\left\langle\hat{\sigma}_{12}^{\dagger} \hat{a}^{\dagger} \hat{a}\right\rangle-i g_{1}\left\langle\hat{a}^{\dagger} \hat{a} \hat{a}^{\dagger} \hat{\sigma}_{2 e}\right\rangle+i g_{2}\left\langle\hat{\sigma}_{1 e}^{\dagger} \hat{a} \hat{a}^{\dagger} \hat{a}\right\rangle \\
& -\left(\boldsymbol{\kappa}+\frac{1}{2}\left(\gamma_{10}+\gamma_{21}\right)\right)\left\langle\hat{\sigma}_{12}^{\dagger} \hat{a}^{\dagger} \hat{a}\right\rangle
\end{aligned}
$$

We restrict ourselves to the subspace of maximal one photon in the cavity. This is justified since the cavity decay rate $\boldsymbol{\kappa}$ is several orders of magnitude larger than the coupling rates $g_{i}$ (see below). Furthermore the cavity rate is also several orders of magnitude larger than the excitation rate at typical pump powers (see Sect. 3.2). In the subspace of maximal 1 photon $\hat{a}^{\dagger} \hat{a} \hat{a}^{\dagger}=\hat{a}^{\dagger}$ and $\hat{a} \hat{a}^{\dagger} \hat{a}=\hat{a}$ holds. Adiabatic elimination of expressions like $\left\langle\hat{\sigma}_{i j}^{\dagger} \hat{a}^{\dagger} \hat{a}\right\rangle$ leads for these terms to

$$
i\left\langle\hat{\sigma}_{i j}^{\dagger} \hat{a}^{\dagger} \hat{a}\right\rangle=c_{1}\left\langle\hat{a}^{\dagger} \hat{\sigma}_{j e}\right\rangle+c_{2}\left\langle\hat{\sigma}_{i e}^{\dagger} \hat{a}\right\rangle
$$

where the absolute values for $c_{1}$ and $c_{2}$ are on the order of $\mathrm{MHz}$ or smaller when taking into account the order of magnitude of the different parameters (for typical values of these see later on). When substituting the expressions in Eqs. 2.90 and their complex conjugates leads in principle to a system of six coupled equations. However, the substituted expressions are several orders of magnitude smaller than the other terms in Eqs. 2.90, such that all terms of the form $\left\langle\hat{\sigma}_{i j}^{\dagger} \hat{a}^{\dagger} \hat{a}\right\rangle$ and their complex conjugates can directly be dropped. Adiabatic elimination of the coherences finally leads to:

$$
\begin{aligned}
\frac{d}{d t}\left\langle\hat{a}^{\dagger} \hat{a}\right\rangle & =-\left(\boldsymbol{\kappa}+R_{0}\right)\left\langle\hat{a}^{\dagger} \hat{a}\right\rangle+\left(R_{0}+R_{1}+R_{2}\right)\left\langle\hat{\sigma}_{e e}\right\rangle \\
\frac{d}{d t}\left\langle\hat{\sigma}_{e e}\right\rangle & =-\left(\boldsymbol{\gamma}+R_{0}+R_{1}+R_{2}\right)\left\langle\hat{\sigma}_{e e}\right\rangle+R_{0}\left\langle\hat{a}^{\dagger} \hat{a}\right\rangle
\end{aligned}
$$

where

$$
\begin{aligned}
R_{0} & =\frac{4 g_{0}^{2}}{\kappa+\gamma+\gamma^{\star}} \frac{1}{1+\left(\frac{2 \delta_{0}}{\kappa+\gamma+\gamma^{\star}}\right)^{2}} \\
R_{1} & =\frac{4 g_{1}^{2}}{\kappa+\gamma+\gamma_{10}+\gamma^{\star}} \frac{1}{1+\left(\frac{2 \delta_{1}}{\kappa+\gamma+\gamma_{10}+\gamma^{\star}}\right)^{2}} \\
R_{2} & =\frac{4 g_{2}^{2}}{\kappa+\gamma+\gamma_{21}+\gamma^{\star}} \frac{1}{1+\left(\frac{2 \delta_{2}}{\kappa+\gamma+\gamma_{21}+\gamma^{\star}}\right)^{2}} \\
\gamma & =\gamma_{1}+\gamma_{2}+\gamma_{3},
\end{aligned}
$$

where $\delta_{0}, \delta_{1}, \delta_{2}$ : detunings between optical transition and cavity mode.

These rate equations (2.93a) and (2.93b) describe a simplified model of two coupled boxes (cf. [172]), an emitter box and a cavity box, with $\gamma$ the loss rate from the emitter box and $\boldsymbol{\kappa}$ the loss rate of the cavity box. The boxes are coupled with the diffusion 
rates $R_{0}, R_{1}, R_{2}$, where the last two rates are only one-directional (see Fig. 2.12(b)). In the case where $\boldsymbol{\kappa} \gg \gamma, R_{i}$ the efficiency on each transition, defined as ratio of the emission coupled into the cavity mode with respect to the total emission is:

$$
P_{i}=\frac{R_{i} / \gamma}{1+\sum_{j=0}^{2} R_{j} / \gamma}, \quad i=0,1,2 .
$$

If the cavity is tuned to any arbitrary wavelength within the NV emission spectrum, the total emission into the cavity mode is the sum of contributions from ZPL and all PSB:

$$
P_{\text {tot }}(\lambda)=\sum_{i=0}^{2} P_{i}(\lambda)=\frac{\sum_{i=0}^{2} R_{i}}{\gamma+\sum_{j=0}^{2} R_{j}} .
$$

The generalization of Eqs. 2.93-2.96 to $n$ vibrational ground-states was already given in the overview-section of the model in Eqs. 2.82-2.85.

\subsection{Numerical simulations}

In this section the theory presented in 2.4 is used for numerical evaluation. In the first part the results of the rate model are presented and discussed. In the second part we also evaluate the full master equation numerically using the quantum optics toolbox for Matlab by Tan [189] with two different goals. First, it's to validate the approximations made to derive the simplified rate model for the room temperature case. Second, the numerical evaluation of the full master equation also allows exploring the regime where the approximation might not be valid any more, i.e. in the case of a NV center cooled to cryogenic temperatures. We start this second part with overview over the working principle of the methods used by the quantum optics toolbox before we reproduce the results from the rate model. The final part contains the predictions for the cryogenic case.

\subsubsection{Nitrogen-vacancy center at room temperature - rate model}

We want to use equations 2.83 and 2.84 to finally determine the total emission rate into the cavity with respect to the total emission rate (cf. Eq. 2.85). All input parameters for the NV center, i.e. the energies of the vibronic ground states and excited state $E_{i}$, the spontaneous decay rates $\gamma_{i}$ the pure dephasing rate $\gamma^{\star}$ and the ground state relaxation rates $\gamma_{i, i-1}$, are determined from fitting the spectra of an actual measured single NV center's emission into free-space [see Fig. 2.11(a)]. A detailed discussion of the spectra of single NV-centers, deduction of the relevant parameters and comparison to the one-phonon Huang-Rhys model will follow in the next chapter (Sect. 3.3). For now the obtained parameters are simply summarized in table 2.1. Analogously, the assumed parameters for the cavity are within the range of experimentally observed values, i.e. the radius of curvature of the mirror is set to $R=70 \mu \mathrm{m}$ and the cavity 
length is varied between $l=3-6 \mu \mathrm{m}$. The finesse is calculated according to the measured transmission of the mirror coatings and a typical value for the additional cavity round-trip losses is assumed (see Sect. 4.2.4). From the cavity length and finesse the cavity decay rate is determined by $\boldsymbol{\kappa}=2 \pi \times c /(2 l \mathcal{F})$. The cavity coupling rate $g$ is calculated from equations 2.46 and 2.72 .

\begin{tabular}{|c|c|c|c|c|}
\hline \hline \multicolumn{5}{|c|}{ NV center } \\
\hline $\mathrm{i}$ & $\begin{array}{c}E_{i} \\
{[\mathrm{meV}]}\end{array}$ & $\begin{array}{c}\gamma_{i} \\
{[\mathrm{MHz}]}\end{array}$ & $\begin{array}{c}\gamma^{\star} \\
{[\mathrm{THz}]}\end{array}$ & $\begin{array}{c}\gamma_{i, i-1} \\
{[\mathrm{THz}]}\end{array}$ \\
\hline 0 & 0 & 0.69 & 15 & - \\
1 & 31.8 & 2.42 & 15 & 85 \\
2 & 70.3 & 8.57 & 15 & 82 \\
3 & 124 & 7.57 & 15 & 79 \\
4 & 168 & 6.46 & 15 & 88 \\
5 & 221 & 4.23 & 15 & 65 \\
6 & 275 & 3.03 & 15 & 71 \\
7 & 319 & 1.51 & 15 & 86 \\
$\mathrm{e}$ & 1941 & - & - & - \\
\hline \hline
\end{tabular}

Table 2.1: full list of parameters for the NV centers used in the simulations

The total efficiency is calculated as function of the resonance wavelength of the cavity and plotted in Fig. 2.13 for different longitudinal mode number $n=10-17$, which determines the cavity length by $l=n \times \lambda / 2$. For longer cavities the mode volume increases and thus the coherent coupling $g$ decreases, which reduces the efficiency for increasing longitudinal mode number $n$ as shown in Fig. 2.13. The achievable efficiencies lie in the range of a few percent, which is the ratio of the total (optical) emission from the NV center which is emitted into the cavity and coupled out through the cavity with decay rate $\boldsymbol{\kappa}$. The effective efficiency will be lower as only a fraction of the cavity decay is into the collected output channel, which has not been taken into account yet.

To value these obtained efficiencies it is instructive to relate them to the case where the cavity acts only as a filter. We consider the case where the cavity is in resonance with the ZPL $(\lambda=638 \mathrm{~nm})$ and a cavity length of $l=10 \times \lambda / 2=3.2 \mu \mathrm{m}$. A finesse of $\mathcal{F}=3500$ results in a cavity linewidth of $\delta \nu=13.4 \mathrm{GHz}$. This leads to a spectral filtering of the broad NV emission $\left(\Delta \lambda_{F W H M} \approx 100 \mathrm{~nm}\right)$ by a factor of $\epsilon_{\text {spectral }}=2.5 \times 10^{-4}$. Additionally spatial filtering has also to be taken into account. The collection angle is determined by the numerical aperture (NA) of the fiber, that will be used in these experiments. The typical NA of a fiber is NA $=0.15$. This solid angle covers only the fraction $\epsilon_{\text {spatial }}=1-\sqrt{1-(\mathrm{NA})^{2}}=1.1 \%$ of the half-space. Thus by filtering we expect only the fraction

$$
\epsilon_{\text {filter }}=\epsilon_{\text {spectral }} \times \epsilon_{\text {spatial }}=2.8 \times 10^{-6}
$$




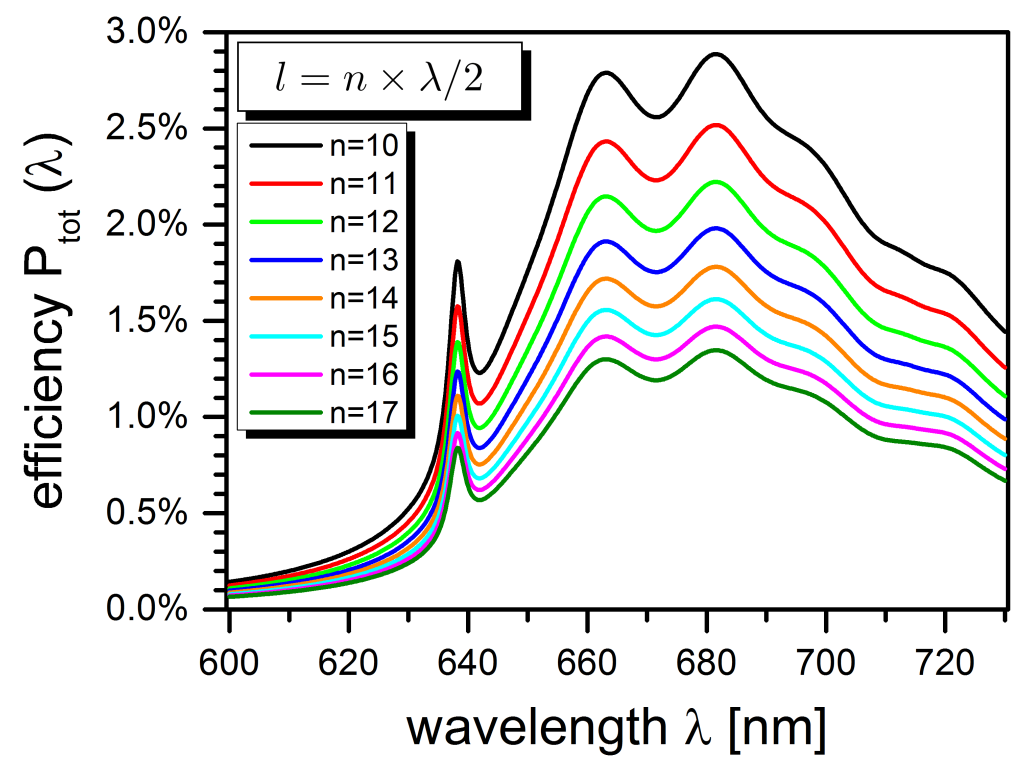

Figure 2.13: Simulated efficiency $P_{\text {tot }}(\lambda)$ as function of the cavity resonance wavelength $\lambda$ for cavities of length $l=n \times \lambda / 2$ for different longitudinal mode number $n=10-17$.

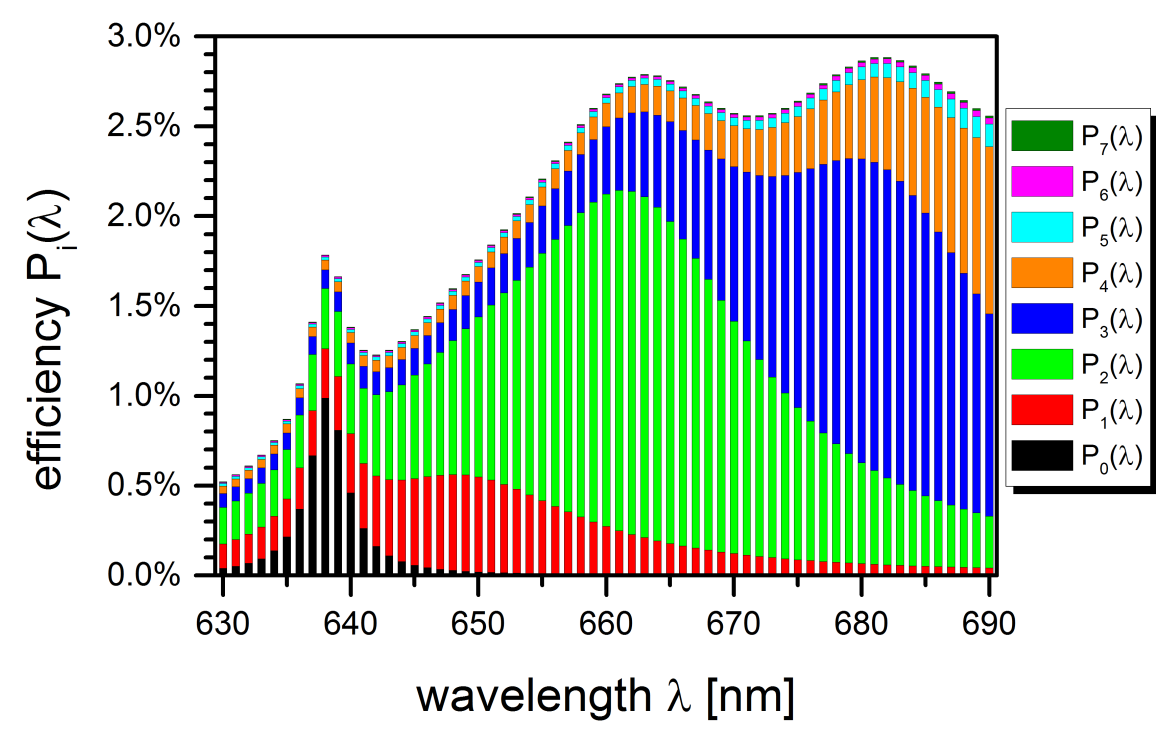

Figure 2.14: Illustration of the contribution of each transition (ZPL + PSB) to the total efficiency $P_{\text {tot }}(\lambda)$. The cavity length is $l=10 \times \lambda / 2$. 
to be collected from the NV center. This is 4 orders of magnitude smaller as the predicted efficiency from the model. Hence coupling to microcavity can have a significant effect, even for a broad-band emitter.

Taking a look at the contribution from the ZPL and the PSB to the total efficiency as illustrated in Fig. 2.14 we notice that at any given cavity resonance wavelength $\lambda$ all sidebands contribute to the emission into the cavity. For instance when the cavity is in resonance with the ZPL, only $54 \%$ of the emission into the cavity can be attributed to the ZPL transition. The rest is from the PSB, which is channeled off-resonantly into the cavity mode very similar to the cavity feeding observed for quantum dots as described above.

\subsubsection{Quantum optics toolbox}

To validate the approximations made to derive the rate model and to explore further parameter range, direct numerical solution of the master equation is necessary. This is done using the quantum optics toolbox for Matlab by Tan [189]. The underlying working principle of the toolbox-functions used here shall now be illustrated.

The master equation in the Lindblad form for some dissipation operator $O$ takes the form:

$$
\frac{d}{d t} \rho=\mathcal{L}[\hat{O}, \rho] \equiv \hat{O} \rho \hat{O}^{\dagger}-\frac{1}{2}\left(\hat{O}^{\dagger} \hat{O} \rho+\rho \hat{O}^{\dagger} \hat{O}\right) .
$$

To write the master equation as a linear equation the matrices are flattened to a vector column by column:

$$
A=\left(\begin{array}{ll}
A_{11} & A_{12} \\
A_{21} & A_{22}
\end{array}\right) \quad \Rightarrow \tilde{A}=\left(\begin{array}{l}
A_{11} \\
A_{21} \\
A_{12} \\
A_{22}
\end{array}\right)
$$

This allows writing $A \rho$ and $\rho A$ in the following way:

$$
\begin{aligned}
& A \rho=\left(\begin{array}{ll}
A_{11} & A_{12} \\
A_{21} & A_{22}
\end{array}\right)\left(\begin{array}{ll}
\rho_{11} & \rho_{12} \\
\rho_{21} & \rho_{22}
\end{array}\right)=\left(\begin{array}{ll}
A_{11} \rho_{11}+A_{12} \rho_{21} & A_{11} \rho_{12}+A_{12} \rho_{22} \\
A_{21} \rho_{11}+A_{22} \rho_{21} & A_{21} \rho_{12}+A_{22} \rho_{22}
\end{array}\right) \\
& \widetilde{A \rho}=\left(\begin{array}{c}
A_{11} \rho_{11}+A_{12} \rho 21 \\
A_{21} \rho_{11}+A_{22} \rho_{21} \\
A_{11} \rho_{12}+A_{12} \rho_{22} \\
A_{21} \rho_{12}+A_{22} \rho_{22}
\end{array}\right)=\underbrace{\left(\begin{array}{cccc}
A_{11} & A_{12} & 0 & 0 \\
A_{21} & A_{22} & 0 & 0 \\
0 & 0 & A_{11} & A_{12} \\
0 & 0 & A_{21} & A_{22}
\end{array}\right)}_{\equiv \operatorname{spre}(A)} \underbrace{\left(\begin{array}{c}
\rho_{11} \\
\rho_{21} \\
\rho_{12} \\
\rho_{22}
\end{array}\right)}_{\tilde{\rho}}
\end{aligned}
$$

and 


$$
\begin{aligned}
& \rho A=\left(\begin{array}{ll}
\rho_{11} A_{11}+\rho_{12} A_{21} & \rho_{11} A_{12}+\rho_{12} A_{22} \\
\rho_{21} A_{11}+\rho_{22} A_{21} & \rho_{21} A_{12}+\rho_{22} A_{22}
\end{array}\right) \\
& \widetilde{\rho A}=\left(\begin{array}{l}
A_{11} \rho_{11}+A_{21} \rho_{12} \\
A_{11} \rho_{21}+A_{21} \rho_{22} \\
A_{12} \rho_{11}+A_{22} \rho_{12} \\
A_{12} \rho_{21}+A_{22} \rho_{22}
\end{array}\right)=\underbrace{\left(\begin{array}{cccc}
A_{11} & 0 & A_{21} & 0 \\
0 & A_{11} & 0 & A_{22} \\
A_{12} & 0 & A_{22} & \\
0 & A_{12} & 0 & A_{22}
\end{array}\right)}_{\equiv \operatorname{spost}(A)} \underbrace{\left(\begin{array}{l}
\rho_{11} \\
\rho_{21} \\
\rho_{12} \\
\rho_{22}
\end{array}\right)}_{\tilde{\rho}},
\end{aligned}
$$

where $\operatorname{spre}(A)$ and $\operatorname{spost}(A)$ define the corresponding $4 x 4$ matrices describing pre- or post-multiplication of $\rho$ with the matrix $A$ in the flattend description.

This allows writing the master equation as linear differential equation:

$$
\frac{d}{d t} \tilde{\rho}=L \tilde{\rho}
$$

The formal solution to this equation is

$$
\rho(t)=\rho(0) \exp (L t)
$$

Numerical evaluation of the exponential series of a matrix is achieved via finding the eigenvalues and eigenvectors of $L$. Once the time dependent density matrix is known, it is straightforward to calculate the operator expectation values by $\langle O(t)\rangle=\operatorname{tr}(\rho(t) O)$. Using the collapse operator describing the cavity decay $C_{\text {cav }}$ and the collapse operators for spontaneous decay $C_{\mathrm{sp}_{i}}$ we can calculate the total efficiency by

$$
P_{\mathrm{tot}}=\frac{\int_{t=0}^{\infty}\left\langle C_{\mathrm{cav}}(t)\right\rangle}{\int_{t=0}^{\infty}\left\langle C_{\mathrm{cav}}(t)\right\rangle+\sum_{i=1}^{n} \int_{t=0}^{\infty}\left\langle C_{\mathrm{sp}_{i}}(t)\right\rangle} .
$$

For numerical evaluation the time is discretized and cut off at sufficiently large values. For the simulation it is further necessary to restrict the photonic Hilbert space to a finite number of photon, which is here restricted to maximal one photon in the cavity. Note that in general this restriction is rather strong and for instance automatically generates anti-bunched light. However, within the assumed cavity parameters the cavity decay rate is much faster than the excitation rate of the NV center. Hence, this restriction is here justified. The initial density matrix is given by the NV center being in the excited state and an empty cavity. 


\subsubsection{Validation of the rate model}

Using the same parameters as for the evaluation with the rate model we calculate the total efficiency now by numerical solution of the full master equation (cf. Eq. 2.104). Figure 2.15 shows the obtained results for both methods which agree very well. Hence the approximations made are well justified for an NV center at room temperature coupled to a microcavity. Here, a longitudinal mode number $n=11$ is used (cavity length $l=n \times \lambda / 2$ ).

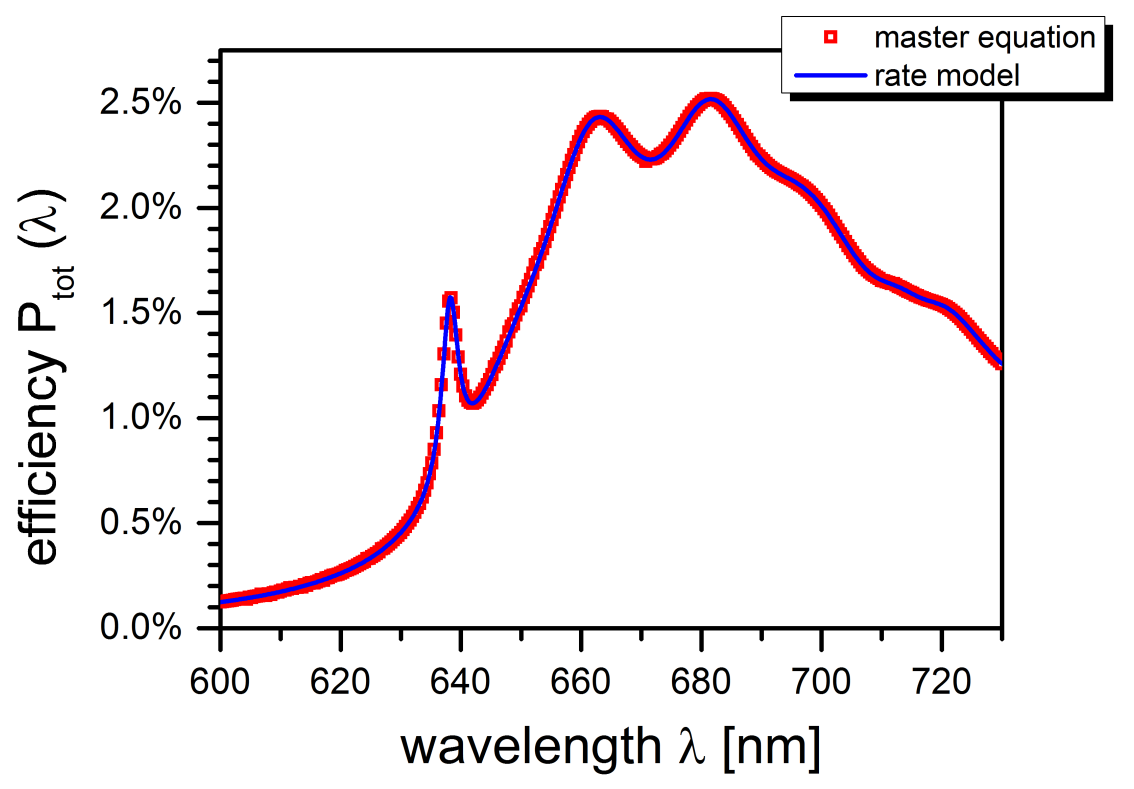

Figure 2.15: Comparison of obtained results using the rate model and numerical solution of the full master equation

\subsubsection{Nitrogen-vacancy center at cryogenic temperatures}

We also can use the model to investigate the effect of cavity coupling for an NV center at cryogenic temperatures. Cooling of the NV center will decrease the broadening of the ZPL due to the dynamic Jahn-Teller effect [cf. Fig. 2.8(b)] and thus result in a reduction of the pure dephasing rate $\gamma^{\star}$. Using the solution of the full master equation calculated with the quantum optics toolbox with the cavity parameter as before, that is a cavity length of $l=10 \times \lambda / 2$ and radius of curvature of the concave mirror of $R=70 \mu \mathrm{m}$, Fig. 2.16(a) displays the total efficiency $P_{\text {tot }}\left(\lambda_{\mathrm{ZPL}}\right)$ as function of the dephasing rate $\gamma^{\star}$ when the cavity is in resonance with the NV center's ZPL wavelength. By fitting the time-dependent decay of the excited state population with an exponential function the lifetime is determined, which is also plotted in Fig 2.16(a). A transition from the dephasing assisted cavity feeding regime to the Purcell regime is observed when 
the dephasing rate becomes comparable to the cavity decay rate $(\boldsymbol{\kappa}=80 \mathrm{GHz})$. The lifetime changes by a factor of 3 from $28.6 \mathrm{~ns}$ to $9.8 \mathrm{~ns}$. The ratio of emission into the cavity with respect to the total emission rate rises from $2.3 \%$ at a dephasing rate of $\gamma^{\star}=10 \mathrm{THz}$ to $66 \%$ at a dephasing rate of $\boldsymbol{\gamma}^{\star}=1 \mathrm{GHz}$. This efficiency is no more limited by the dephasing $\boldsymbol{\gamma}^{\star}$, but the cavity decay rate $\boldsymbol{\kappa}$ instead and determined by the $\beta$-factor (cf. Eq. 2.69). With a coherent coupling rate of the ZPL transition of $g_{0}=1.2 \mathrm{GHz}$ and the emitter decay rate of $\gamma=35 \mathrm{MHz}$ we find

$$
\begin{aligned}
F_{p} & =\frac{4 g_{0}^{2}}{\kappa \gamma}=2.1 \\
\beta & =\frac{F_{p}}{1+F_{p}}=68 \%,
\end{aligned}
$$

which agrees with the results obtained for small dephasing $\gamma^{\star}$.

Cooling of the NV center only slightly affects the emission on the PSB. The width of the PSB sidebands is only slightly reduced because the fast ground-state relaxation is not changed. Thus for a cavity with resonance wavelength in the range of the PSB the rate of emission into cavity is basically unaltered and still determined by the feeding mechanism of the cavity from all PSB transitions. This is illustrated in Fig. 2.16(b) where the total efficiency $P_{\text {tot }}(\lambda)$ as function of the cavity wavelength is plotted both for the room temperature situation with a pure dephasing rate of $\gamma^{\star}=15 \mathrm{THz}$ and for a cooled NV center with a exemplarily dephasing rate of $\gamma^{\star}=100 \mathrm{GHz}$. Whereas for a cavity in resonance with the ZPL wavelength, a dramatic change is observed, cooling of the NV center has only a little effect on the emission rate into a cavity in the PSB wavelength range.

Additionally, we can also use the rate model to calculate the total efficiency $P_{\text {tot }}\left(\lambda_{\text {ZPL }}\right)$ and the decay time by $\tau=\left(\gamma+\sum_{i} R_{i}\right)^{-1}$. As Fig. 2.16(a) clearly illustrates, the approximations made by the rate model are still valid for these parameter range. The difference in the total efficiency $\Delta P_{\text {tot }}\left(\lambda_{\mathrm{ZPL}}\right)$ between the results obtained by the solution of the master equation and the rate model is plotted in Fig. 2.16(d). It is not surprising that they agree when comparing the cavity decay $\kappa=80 \mathrm{GHz}$ with the coherent coupling rate of the ZPL $g_{0}=1.2 \mathrm{GHz}$. The cavity decay is much faster than the coherent exchange of excitation between NV center and cavity and therefore the coherences are negligible and the approximation of the master equation by the rate equation well justified. Even at the limit of feasible parameters for the cavity with a cavity length of $l=2 \mu \mathrm{m}$, radius of curvature of $R=30 \mu \mathrm{m}$ and a cavity finesse of $\mathcal{F}=50000$, where the cavity decay rate $\boldsymbol{\kappa}=9.8 \mathrm{GHz}$ becomes comparable to the coherent coupling rate of the ZPL $g_{0}=2.1 \mathrm{GHz}$, coherent effects still are not significant. As shown in Figs. 2.16(c) and (d) the rate model still accurately describes the dynamics. At low temperatures, with a pure dephasing rate $\gamma^{\star}=1 \mathrm{GHz}, 97.6 \%$ of the emission is directed into the cavity mode when the cavity is in resonance with the ZPL. The lifetime is reduced to $0.7 \mathrm{~ns}$, which corresponds to a Purcell factor of $F_{p}=4 g_{0}^{2} /(\boldsymbol{\kappa} \gamma)=51$. The predicted efficiency by the rate model is in this case $0.3 \%$ higher than the calculated 
efficiency from the master equation, which relative to the absolute value of the efficiency is negligible.

As discussed above the linewidth of the ZPL emission is not only limited by pure dephasing, but also by spectral diffusion. Whereas pure dephasing diminishes for reduced temperature, spectral diffusion will remain unaltered and will especially in NDs limit the linewidth at cryogenic temperatures (see also Sect. 3.3). For a strong enhancement the detuning between cavity resonance wavelength and ZPL wavelength needs to be smaller than the cavity linewidth or pure dephasing rate as can be seen in Fig. 2.16(b). At cryogenic temperatures, where dephasing becomes negligible, it will therefore be

(a)

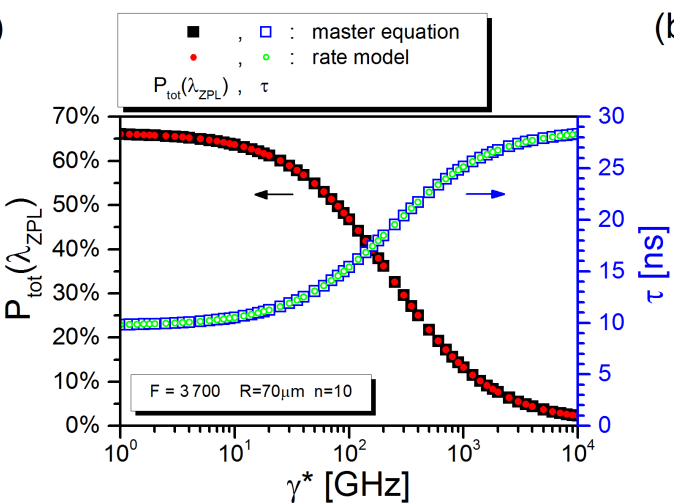

(c)

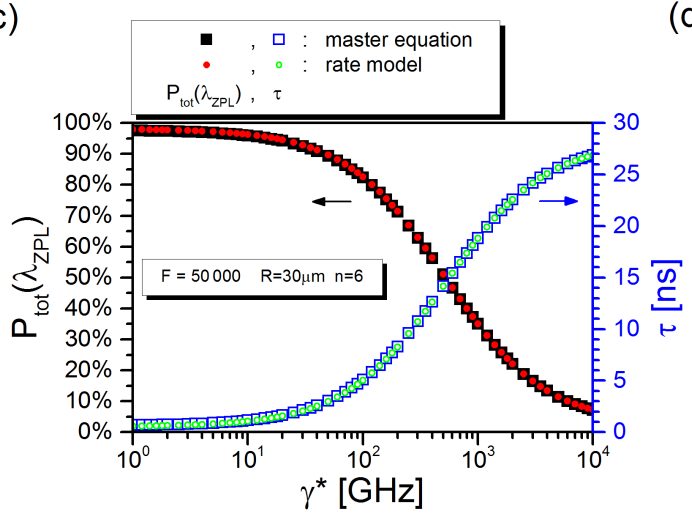

(b)

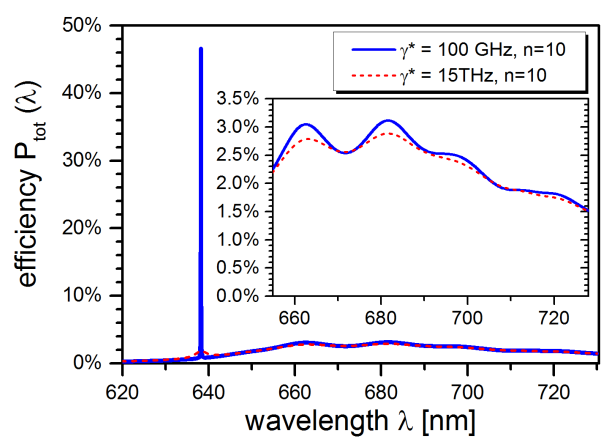

(d)

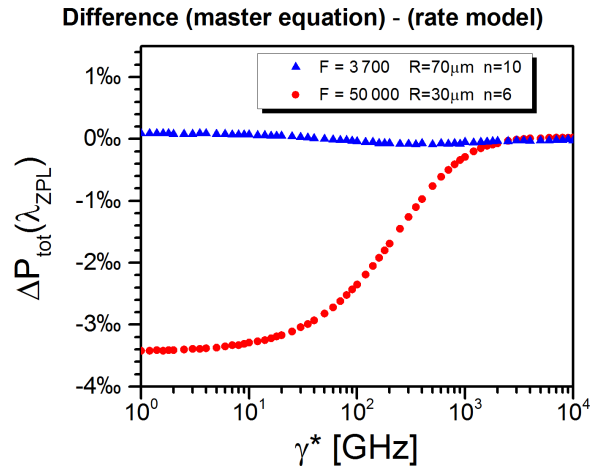

Figure 2.16: (a), (c) Total efficiency, i.e. ratio of emission coupled out through cavity compared to total emission of NV center (full markers) and lifetime of the excited state (empty markers) as function of the pure dephasing rate $\gamma^{\star}$ calculated both via rate model (circles) and solution of the full master equation (squares) for realized cavity parameters in (a) and ideally achievable parameters in (c). (b) Total efficiency as function of the cavity resonance wavelength assuming a dephasing rate of $\gamma^{\star}=100 \mathrm{GHz}$ (blue solid line) and cavity parameters as in (a) (red dashed line). The inset shows a zoom of the PSB wavelength range. (d) Difference of the calculated total efficiency by the two methods (solution of master equation and rate model) for the two cases shown in (a) (blue triangles) and (c) (red circles) 
important to match the cavity design to the width covered by spectral diffusion.

Numerical evaluation of the full master equation also allows investigating the onset of the strong coupling regime. We here assume a realistic cavity length of $3.2 \mu \mathrm{m}$ with one plane mirror and a fiber mirror with radius of curvature of $15 \mu \mathrm{m}$. This determines the mode volume and hence the coherent coupling rate. For the ZPL we obtain $g_{0}=1.81 \mathrm{GHz}$. In order to enter the strong coupling regime we need to reduce the cavity decay rate by increasing the cavity finesse. To achieve the limiting case $\boldsymbol{\kappa}=g_{0}$ one needs a finesse $\mathcal{F}=(\pi c) /(\boldsymbol{\kappa} l)=1.6 \times 10^{5}$. In order not to be limited by the emitter the pure dephasing $\gamma^{\star}$ has also to be smaller than the coherent coupling rate as we will show below.

We investigate two cases, first $\gamma^{\star}=1 \mathrm{GHz}$ and second $\gamma^{\star}=10 \mathrm{GHz}$. We assume the cavity to be in resonance with the ZPL. The cavity has a constant mode volume of $V_{\text {mod }}=3.1 \mu \mathrm{m}^{3}=12 \lambda_{\mathrm{ZPL}}^{3}$. For a number of discrete values of the finesse we calculate

(a)

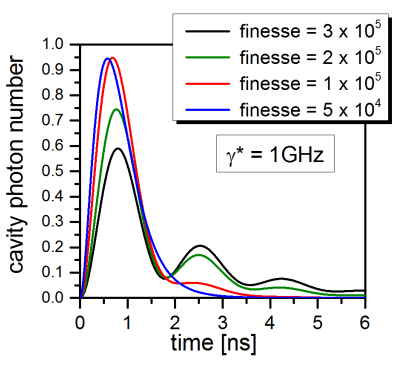

(d)

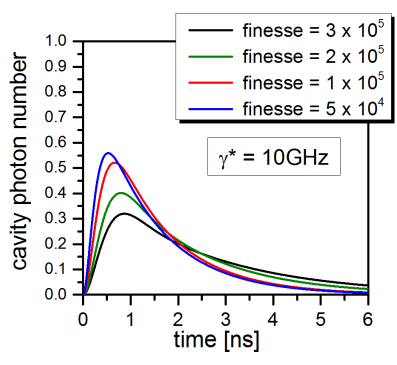

(b)

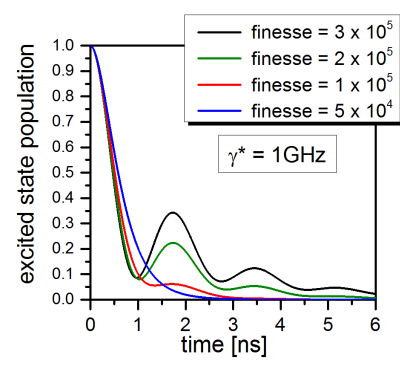

(e)

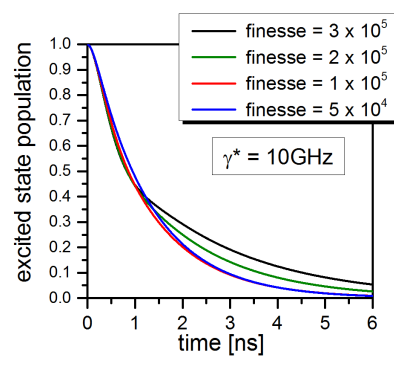

(c)

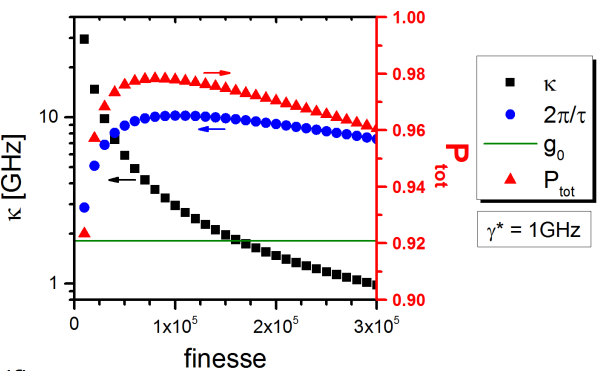

(f)

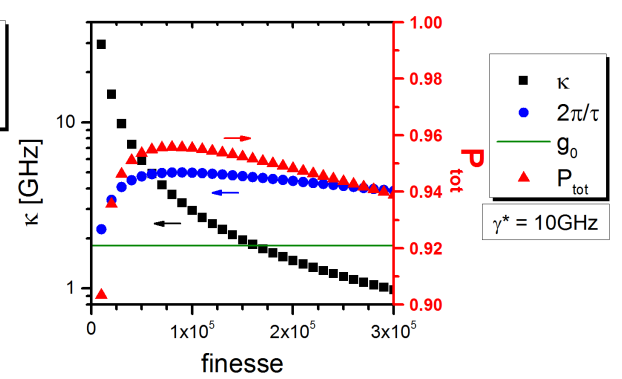

Figure 2.17: Time evolution of the cavity photon number $[(\mathrm{a}) \&(\mathrm{~d})]$ and excited state population [(b) \& (e)] for values of the cavity finesse of $\mathcal{F}=5 \times 10^{4}, 1 \times 10^{5}, 2 \times 10^{5}, 3 \times 10^{5}$ assuming a pure dephasing rate of $\gamma^{\star}=1 \mathrm{GHz}$ or $\gamma^{\star}=10 \mathrm{GHz}$, respectively. Fitting with an exponential decay allows to determine the excited state lifetime $\tau$. (c) \& (f) Cavity decay rate $\boldsymbol{\kappa}$ (black squares), lifetime limited linewidth $2 \pi / \tau$ (blue circles) and coherent coupling rate of ZPL transition $g_{0}$ (green horizontal line) as function of the finesse. Further the total efficiency $P_{\text {tot }}$, i.e. the ratio of photons coupled out through the cavity with respect to total emitted photons, is also plotted (red triangles). The pure dephasing rate is $\gamma^{\star}=1 \mathrm{GHz}$ in (c) and $\gamma^{\star}=10 \mathrm{GHz}$ in (f). 
the time evolution of the cavity photon number proportional to $\left\langle\hat{a}^{\dagger} \hat{a}\right\rangle$ and the excited state population proportional to $\left\langle\hat{\sigma}_{e e}\right\rangle$. They are shown in Fig. 2.17(a),(b) for a dephasing rate of $\gamma^{\star}=1 \mathrm{GHz}$ for values of the finesse of $\mathcal{F}=5 \times 10^{4}, 1 \times 10^{5}, 2 \times 10^{5}, 3 \times 10^{5}$. In the cases where $g_{0}>\boldsymbol{\kappa}, \gamma^{\star}$ oscillations in the populations are observed as is expected for the strong coupling regime. If the dephasing rate is increased to $\gamma^{\star}=10 \mathrm{GHz}$ these oscillations are damped out as shown in Fig. 2.17(d),(e). The dephasing is too fast in order for the coherences to persist. However, we still see an increase in excited state lifetime for increased finesse due to non-coherent re-absorption of the cavity photon by the emitter before the photon leaves the cavity.

We can fit the excited state decay with an exponential function to determine an effective emitter lifetime. (Note that in case of strong coupling this fit follows the over the oscillations averaged population and is not the envelope.) The lifetime-limited linewidth is determined by

$$
\Delta \nu_{\tau}=2 \pi / \tau
$$

In Fig. 2.17(c) the cavity decay rate $\boldsymbol{\kappa}$, the coherent coupling rate for the ZPL transition $g_{0}$ and the lifetime-limited linewidth $\Delta \nu_{\tau}$ as function of finesse are plotted for a dephasing rate $\gamma^{\star}=1 \mathrm{GHz}$. As mentioned above at a finesse $\mathcal{F}=1.6 \times 10^{5}$ the coherent coupling rate $g_{0}$ becomes larger than the cavity decay rate $\boldsymbol{\kappa}$. Already at a much lower finesse of $\mathcal{F} \approx 4 \times 10^{4}$ the cavity decay rate becomes smaller than the lifetime limit. This means that the linewidth of the photons is entirely determined by the cavity and we observe Fourier-limited photons from the cavity. The efficiency $P_{\text {tot }}$, which is also plotted in Fig. 2.17, is largest at a finesse of $\mathcal{F}=8 \times 10^{4}$ and equals $P_{\text {tot }_{\max }}=97.83 \%$. This is the ideal working point for use as SPS, that emits indistinguishable photons with a high efficiency. It is worth mentioning that already for a finesse of $\mathcal{F} \approx 4 \times 10^{4}$, where the lifetime limit is achieved, an efficiency of $P_{\mathrm{tot}}=97.3 \%$ is achieved.

In case of an increased dephasing rate $\gamma^{\star}=10 \mathrm{GHz}$ the maximal efficiency is slightly lowered but still a maximum value of $P_{\text {tot }_{\max }}=95.6 \%$ at a finesse of $\mathcal{F}=8 \times 10^{4}$ is achieved and the lifetime limit is reached at a finesse of $\mathcal{F}=6 \times 10^{4}$. These are parameters that are in reach of experimental realizations. 


\section{Chapter 3}

\section{Single nitrogen-vacancy center in a nanodiamond}

This chapter will discuss the preliminary experiments on nitrogen-vacancy centers in NDs with size $<100 \mathrm{~nm}$, which will be used later on to incorporate into the cavity. This incorporation is achieved by deposition of NDs onto one of the two mirrors, which the Fabry-Perot cavity consists of. NV centers in NDs have some advantages and some disadvantages compared to NV centers in bulk diamond. As the size of the diamonds is much smaller than the emission wavelength, fluorescence light will not be refracted at the diamond-air interface. As a good approximation the effective surrounding dielectric medium of the NV center in a ND can be assumed to be air. This circumvents the problem of total internal reflection, that is relevant for an NV center in bulk diamond: due to the high refractive index of diamond $(n=2.42)$, large amount of the emitted light will not be transmitted through the diamond-air interface, but completely reflected (critical angle $\theta_{\text {tir }}=24.6^{\circ}$ ). This is obsolete for an NV center close to the surface. However, due to the large difference in refractive index, the density of states of the electro-magnetic field is correspondingly higher in diamond than air, and thus an NV center close to the surface will preferentially emit into the bulk material and not towards the collection optics.

It is worth noting that in most practical situations this asymmetric emission also occurs for an NV center in a ND, as the ND is not in free-space but deposited on a substrate. Similar to the bulk case, emission will be preferentially towards the substrate and not towards the collection optics. Nevertheless, this can be circumvented to some extent by positioning the ND on a mirror. This statement is not as straight-forward as it seems. There is interference between directly emitted radiation and light reflected at the mirror emission. The situation is thus very critical to the exact distance between emitter and mirror [190-192]. On a metallic mirror additionally quenching due to coupling to surface plasmons can occur opening the path to non-radiating relaxation channels and therefore reducing the emitters lifetime and quantum efficiency [193-195]. Here we will 
use only dielectric mirrors. It is also worth noting, that the reflectivity of a standard dielectric mirror strongly depends on the angle of the incoming light. Thus, the effect of a mirror as substrate is intuitively often over-estimated. Nevertheless, with proper design of a planar multi-layer system, into which single molecules are incorporated as emitters, Lee et al. [196] were able to collect $96 \%$ of the emitted light. A similar approach is also feasible with a NV center in a ND. The broadband collection efficiency also can be increased using solid immersion lenses [197,198] or nanowires [199].

This highlights one of the advantages of NDs compared to bulk diamonds. In contrast to bulk diamond, they can easily be incorporated into other systems, either via spin coating onto a substrate, or even by selecting a specific ND and precisely placing it with an AFM tip or other kind of tip as already mentioned in the introduction [79,81].

Using NDs has also some disadvantages. Most prominent is strain, which is virtually always present in NDs. This leads to a shift in the level structure as shown in Fig. 2.5. As will be discussed later on, this not only shifts the ZPL position, but the coupling to phonons also slightly varies from one NV center to another, which results in variations of the exact PSB structure. A further disadvantage is the close proximity to the surface. The exact surface termination, which determines the chemical potential, influences the charge state $[150,155,156,200]$. Furthermore, the quantum efficiency of an NV center in a ND can also quite dramatically vary from $10 \%$ to $90 \%$ [201]. Commercially available NDs are fabricated by an HPHT process and lack of the purity which can be reached by CVD diamonds. The ZPL of an NV center in a ND typically shows a large spectral diffusion [93,143]. Currently many efforts to ameliorate the properties of NV center in NDs are undertaken, such as precise control of the surface termination [202-204] and fabricating NDs by a bead assisted sonic disintegration (BASD) method from high quality CVD films $[205,206]$. It is worth noting that NV centers in NDs can reach the properties of NV centers in bulk diamond. For instance, a lifetime limited NV center in a ND has been reported $(13 \mathrm{MHz}$ [14] in bulk, $16 \mathrm{MHz}$ in ND [144]. Note that, the lifetime will always differ between NV centers in bulk diamond and in NDs, as the local density of states is different. Thus, in NDs the lifetime is on the order of 20-30 ns, whereas in bulk the lifetime is approximately $13 \mathrm{~ns}$.

The lack of purity in HPHT diamonds and the proximity of defects in NDs to the surface also limits the spin coherence properties. Without surface treatment the spin coherence time $T_{2}$ of NV centers in HPHT NDs is on the order of a few hundred nanoseconds [207]. Surface treatment can slightly increase this coherence time to the order of microseconds [207]. As before the route for NDs with longer spin coherence times is the use of high quality CVD material from which NDs are fabricated. Trusheim et al. [208] recently showed a spin coherence time of of a single NV center in a nanocrystal of $T_{2}=210 \mu$ s using a reactive ion etching (RIE) method to fabricate NDs from a CVD diamond.

In the first section of this chapter the details of the spin coating process developed, in order to deposit well isolated NDs onto a flat mirror, will be presented. The second part discusses the measurement and analysis of the intensity correlation function, which gives some access to the internal dynamics of the NV center. Fluorescence spectra from 
several different single NDs at room temperature and at cryogenic temperature are shown in the last section. Especially the slight differences from ND to ND will be highlighted.

\subsection{Nanodiamonds on a mirror}

\subsubsection{Spin coating}

During fabrication or when extracted from solution NDs tend to agglomerate [209]. Small NDs, such as fabricated by detonation or shock-wave synthesis with diameter 5-20 nm, build primary agglomerates with size up to $100 \mathrm{~nm}$, which are chemically firmly bonded and can hardly be separated. These primary agglomerates further form several microns sized grape-like structures hold together by electrostatic forces, that can be easily de-agglomerated for instance in solution with a ultrasonic bath [209]. The problem to separate the primary agglomerates does not arise for larger NDs fabricated by a HPHT process. They only experience the easily separable agglomeration mediated by electrostatic forces. In water, the separated NDs form a colloidal stable suspension, where they remain de-agglomerated. However, this is critical on the $\mathrm{pH}$-value and concentration of electrolytes in the water [209]. Colloidal suspensions are also possible in other solvents such as DMSO (dimethyl sulfoxide) or ethanol [209].

In this work commercially available NDs fabricated by a HPHT process are used, which are sold de-agglomerated in water (Microdiamant, liquid diamond, MSY 0-0.1). The diameters of the NDs are smaller than $100 \mathrm{~nm}$ and have a median of $60 \mathrm{~nm}$. These NDs contain, without further processing, between 0 - $4 \mathrm{NV}$ centers and the chance of finding a ND with a single NV is sufficiently high. As will be shown below, approximately $10 \%$ of the NDs visible in a confocal microscope image contain exactly one NV center.

To deposit the NDs onto the mirror we use spin coating. A drop (approximately $15 \mu \mathrm{l}$ ) of the solution containing the NDs is dropped onto the substrate. Rotating the mirror yields a thin film of the solution. The mirror is kept rotating until all solvent is evaporated and only the NDs remain. Using only water as solvent is not ideal. The surface of a dielectric mirror is often slightly hydrophobic. Spin coating of a water solution will lead to a inhomogeneous film (formation of small droplets during evaporation) or even none at all, as the surface is completely de-wetted before the water is evaporated. In the first case agglomeration of NDs is observed, and in the second case no NDs at all will be deposited on the mirror.

There are two strategies possible: either to treat the mirror surface in order to make it hydrophilic, or to use a different solvent. We focus on the second approach and use a mixture of water and methanol with a ratio of $1: 2$, containing additionally a small concentration of the polymer Polyvinylpyrrolidone (PVP, average mol weight 360,000). The starting solution, as obtained from Microdiamant, has a concentration of 100 carat $/ \mathrm{Kg}$ of NDs. The final concentration of NDs used is either 1 carat $/ \mathrm{Kg}$ or 
(a)

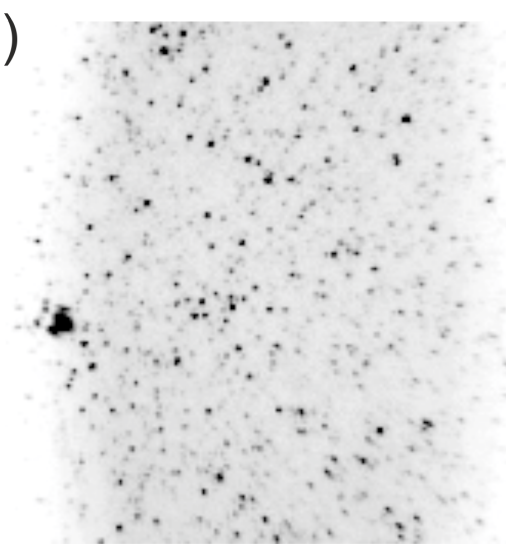

(b)

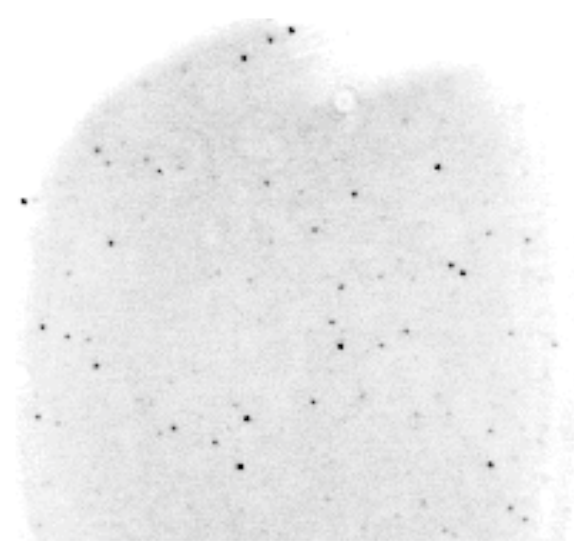

Figure 3.1: Inverted dark field microscope image of mirrors spin-coated with a solution of NDs solved in a mixture of water and methanol with concentrations in (a) 1 carat/Kg or (b) 0.1 carat $/ \mathrm{Kg}$. The mirror is illuminated from the side with white light. A $100 \mathrm{x}$ microscope objective with a numerical aperture of $\mathrm{NA}=0.8$ collects the light scattered by the NDs which is imaged onto a CCD chip. The shown image region is approximately $(150 \mu \mathrm{m})^{2}$ each in size.

0.1 carat $/ \mathrm{Kg}$. The first step is to dilute the original diamond solution with additional water. This ensures that the diamonds remain de-agglomerated, which when directly diluted in another solvent would not necessarily be the case. Second, methanol is added, that already contains PVP with a concentration of $0.1 \% M$. The polymer stabilizes the colloidal suspension and prevents agglomeration. Methanol has the advantage to evaporate quickly under ambient condition. Furthermore, evaporation of water in the mixture with methanol is also accelerated, due to momentum transfer between water and methanol molecules. Figure 3.1 shows a microscope image of two mirrors spincoated with the two concentrations mentioned above, i.e. 1 carat $/ \mathrm{Kg}$ or 0.1 carat $/ \mathrm{Kg}$. This yields well separated NDs. For all samples, spin coating is performed with a rotational frequency of $2500 \mathrm{U} / \mathrm{min}$.

\subsubsection{Confocal microscopy of the nanodiamonds on a mirror}

We use a home-built confocal microscope which is shown in Fig. 3.2. We have two options, (a) either to excite and collect with the same microscope objective using a dichroic mirror or (b) to use a second microscope objective and excite through the substrate. As we use dielectric mirrors as substrate, the mirror in the second case needs to be transparent for the excitation wavelength.

Imaging any dielectric mirror with a scanning confocal microscope shows a nonlocalized high fluorescence rate everywhere (several $10^{5}$ Counts/s detected on a avalanche photo diode (APD) in the spectral window of $650-750 \mathrm{~nm}$ ). This has been observed for mirrors from different manufacturers. The exact fluorescence rate varies from mirror to mirror. When the same spot on the mirror is continuously excited, the fluorescence 
(a)
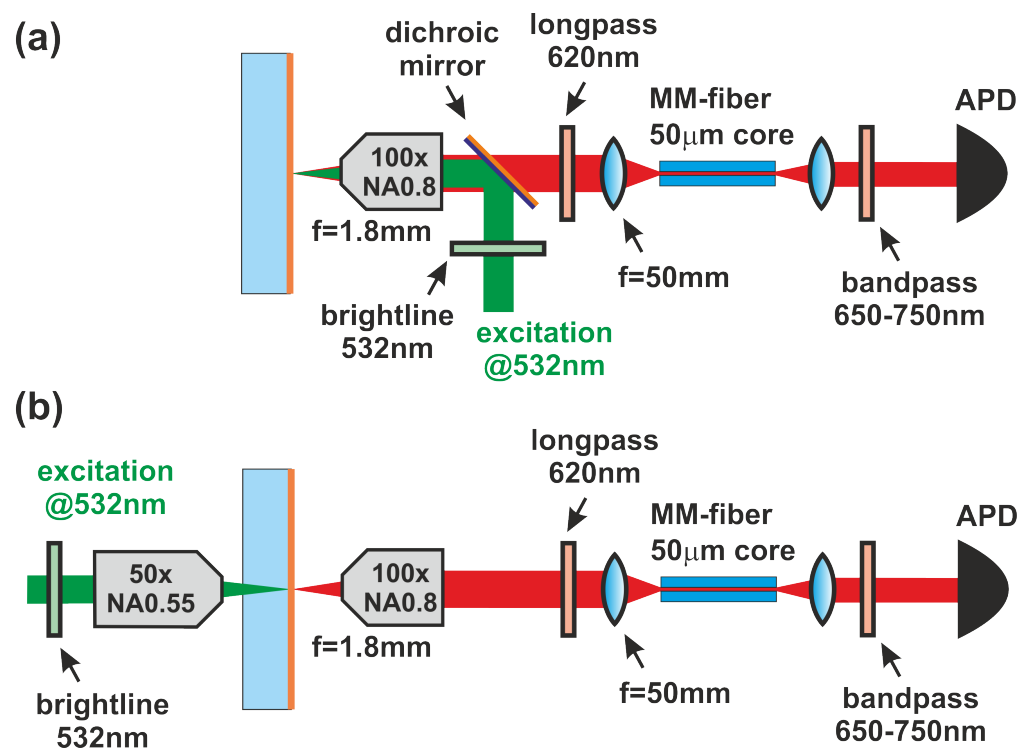

(a) Confocal microscope that consists of a microscope objective (Olympus LMPLFLN, magnification $100 x$, N.A. 0.8 , focal length $f=1.8 \mathrm{~mm}$, working distance $3.4 \mathrm{~mm}$ ) and a multimode fiber (core diameter of $50 \mu \mathrm{m}$ ) acting as pinhole, onto which the collected fluorescence is focused with a achromatic doublet lens (focal length $f=50 \mathrm{~mm}$ ). The excitation laser with a wavelength of $532 \mathrm{~nm}$ is coupled in through the same microscope objective using a dichroic mirror. A longpass and a bandpass filter are used to block the excitation laser in the detection path. For the scans the fluorescence from the fiber is recorded with an APD (MPD PDM PD5CTC). As substrate we use a dielectric mirror. (b) Alternative excitation through a second microscope objective (Mitutoyo MPlanApo, magnification 50x, N.A. 0.55, focal length $f=4 \mathrm{~mm}$, working distance $13 \mathrm{~mm}$ ) and collection optics as in (a). The mirror coating needs to be transparent for the excitation wavelength at $532 \mathrm{~nm}$.

diminishes within seconds by at least one order of magnitude. This bleaching becomes faster with higher excitation power. Thus, to yield a suitable signal to background ratio in order to observe the emission from a single NV centers in NDs, the region of interest on the mirror is first scanned with the excitation laser (wavelength $532 \mathrm{~nm}$ ) focused onto the mirror surface to a spot of approximately $1 \mu \mathrm{m}^{2}$ with typical powers of $10-50 \mathrm{~mW}$. Figure 3.3(a) shows the effect of such a bleaching scan: the image shows a scan of 60x60 pixels where the inner region of 20x20 Pixels has first been bleached. The origin of this fluorescence is not fully understood. It is most likely due to insufficiency of oxygen in the amorphous dielectric layers $\left(\mathrm{SiO}_{2}, \mathrm{Ta}_{2} \mathrm{O}_{5}\right)[210,211]$.

Once the mirror is bleached, the NV centers are clearly visible by confocal microscopy as illustrated in Fig. 3.3(b), that shows a scan of the mirror with densely spin coated NDs. As the mirror is transparent for the excitation wavelength, excitation of the NV center is possible from both directions, i.e. from the front through the same 
microscope objective, that collects the fluorescence, as well as through the plane mirror with a second microscope objective. Figure 3.3(c) and (d) show a confocal scan of the same area using this two excitation options. The only difference is that excitation through the plane mirror leads to a slightly larger spot size on the mirror surface and therefore with the same laser power to a lower intensity, which results in less fluorescence. There are two reasons for this larger spot size. First, the N.A. of the second microscope objective is lower and second, focusing through the $2 \mathrm{~mm}$ thick fused silica, that the mirror consists of, leads to spherical aberrations.

To yield well separated NDs, which can later on be independently coupled to a cavity, we use a lower concentration of 0.1 carat $/ \mathrm{Kg}$. Figure 3.4 shows four adjacent confocal microscope scans, each $80 x 80(\mu \mathrm{m})^{2}$ in size. The 41 marked spots have been investigated further. For each spot first a detail-scan with higher resolution, as shown exemplarily in Fig. 3.5, is performed to precisely position the ND in the laser focus. This

(a)

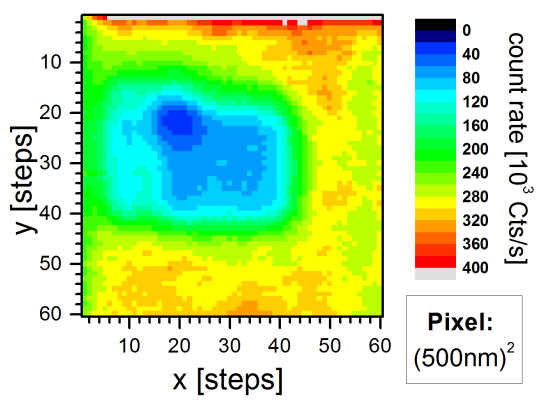

(c)

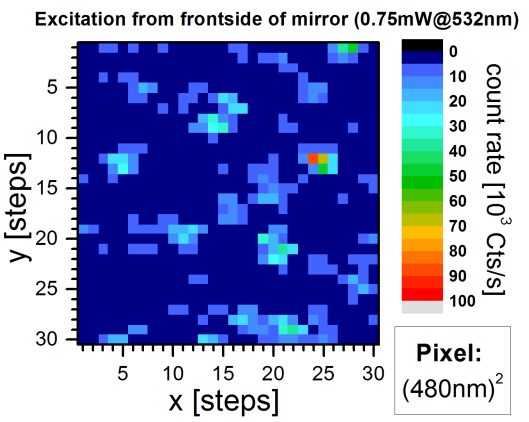

(b)

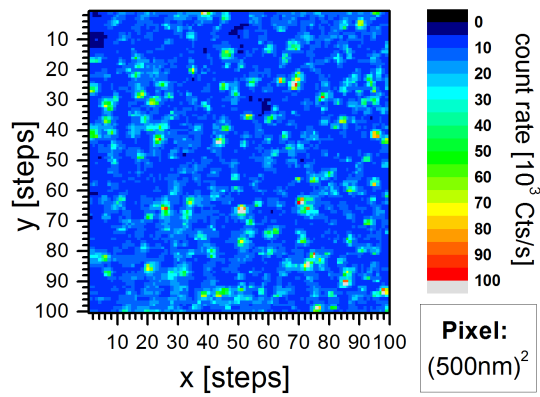

(d)

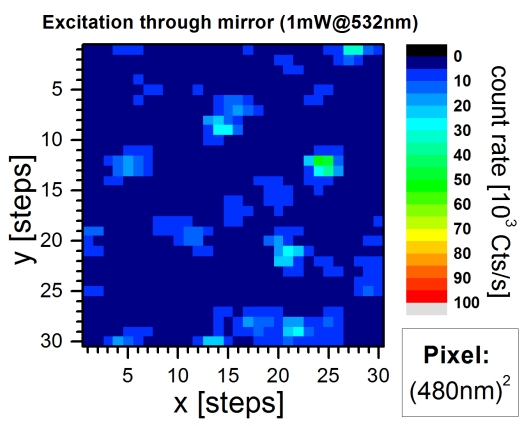

Figure 3.3: (a) Confocal microscope scan of a mirror with excitation at $532 \mathrm{~nm}$ with $1 \mathrm{~mW}$ in front of the microscope objective where the inner region (20x20 pixels) has first been bleached with $4.5 \mathrm{~mW}$ at $532 \mathrm{~nm}$. The low fluorescence around pixel $(20,20)$ is due to longer illumination before and after the bleaching scan. There are no NDs spin coated onto the mirror yet. (b) Confocal microscope image of a mirror spin coated a solution with a concentration of 1 carat $/ \mathrm{Kg}$ NDs. (c) and (d) Confocal microscope image of the same region where in (c) excitation and collection of the fluorescence is through the same microscope objective in front of the mirror and in the $(\mathrm{d})$ excitation is through the plane mirror with a second microscope objective. 
also reveals the spot size of the excitation laser on the mirror. Fitting the horizontal and vertical cross section through the maximum of the detailed scan yields a waist of $0.9 \mu \mathrm{m}$ as shown in Fig. 3.5(b). Note that the spot-size of the excitation laser on the mirror is larger compared to the resolution of the collection path due to the lower N.A. of the microscope objective used for excitation compared to the objective used for collection and the induced spherical aberrations. Next, of each of the marked emitters in Fig. 3.4 the spectra and the intensity correlation is recorded. The measurements of the NDs containing a single NV centers will be discussed in the following sections.
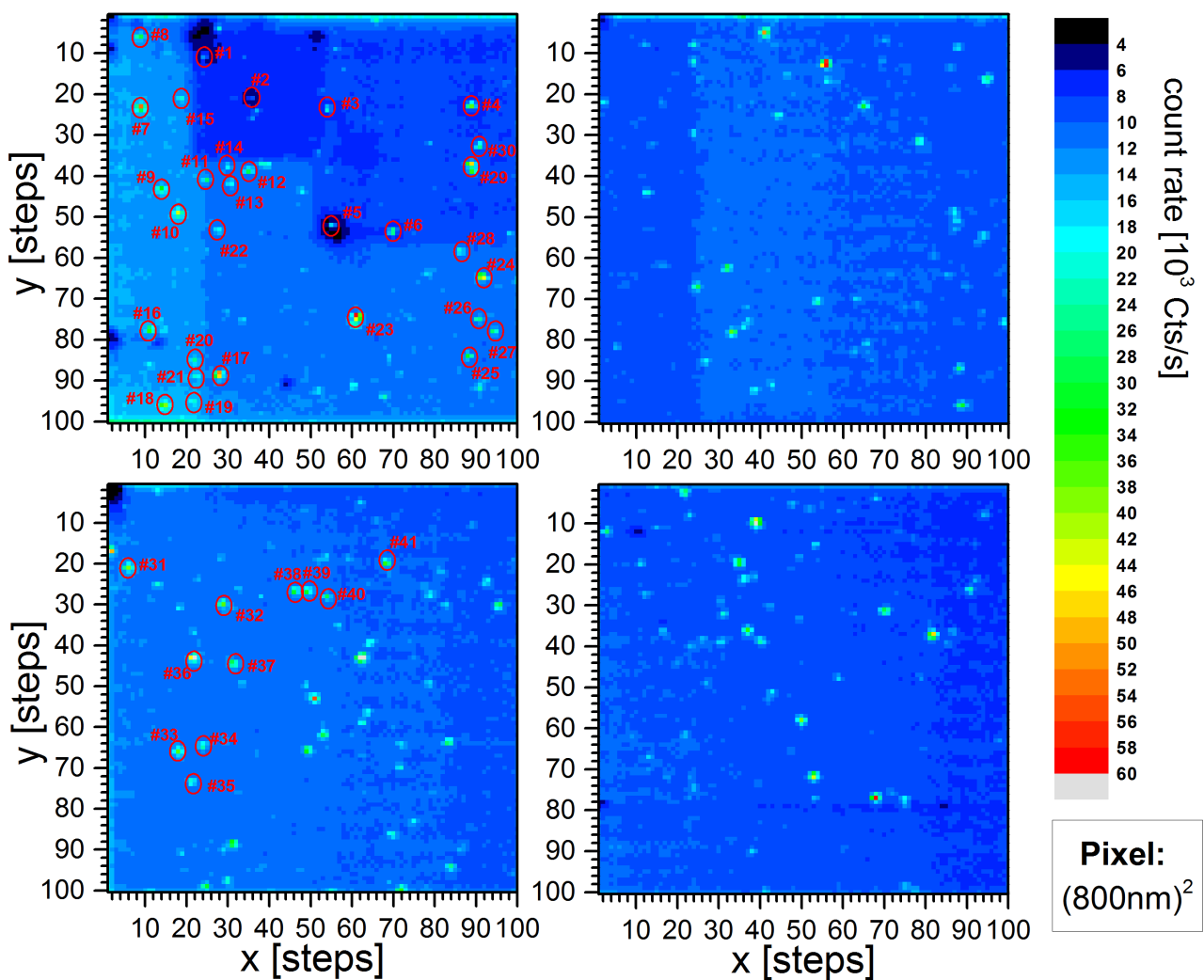

Figure 3.4: Four adjacent scanning confocal microscope images of a mirror spin coated with a solution containing 0.1 carat $/ \mathrm{Kg}$. The marked fluorescent spots are investigated further. 
(a)

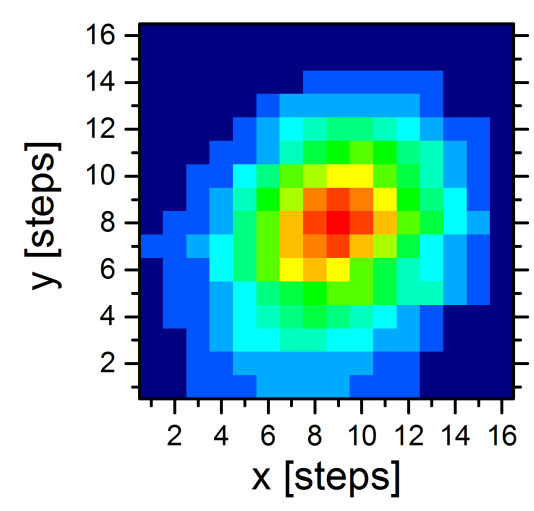

(b)

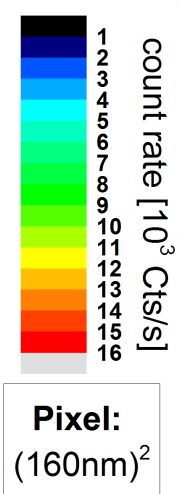

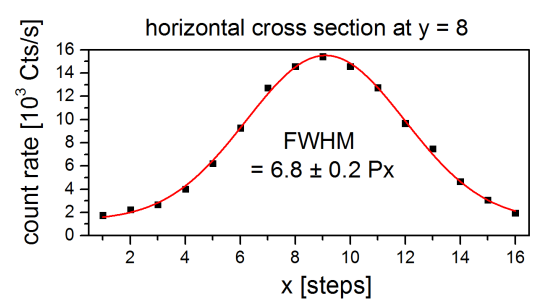

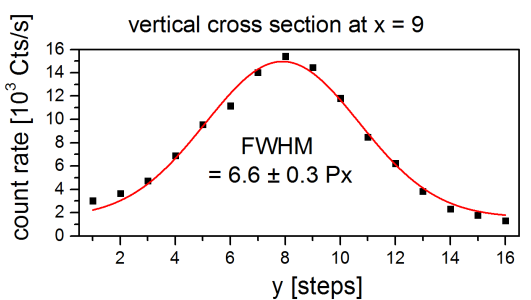

Figure 3.5: (a) A detailed scan with higher resolution of one of the ND (\#41 of Fig. 3.4). Excitation is focused through the plane mirror $(50 \mathrm{x}, \mathrm{N} . \mathrm{A} .=0.55)$ and the fluorescence is collected with a second microscope objective $(100 x$, N.A. $=0.8)$ in front of the mirror. (b) Horizontal and vertical cross-section through maximum which are fitted with a Gaussian. The FWHMs are 6.6 or 6.8 pixels, respectively. This corresponds to a beam waist (half width at $1 / e^{2}$ ) of $\approx 0.9 \mu \mathrm{m}$. Note that this is the determined by the spot size of the excitation laser on the mirror, which is larger than the collection spot.

\subsection{Intensity correlation of a single nitrogen-vacancy cen- ter}

\subsubsection{Relation between internal population dynamics and intensity correlation function}

Measuring the intensity correlation function defined as

$$
g^{(2)}(\tau)=\frac{\langle: \hat{I}(t+\tau) \hat{I}(t):\rangle}{\left\langle\hat{I}(t)^{2}\right\rangle}
$$

reveals the existence of a single emitter when $g^{(2)}(0)<0.5$ because

$$
g^{(2)}(0)=\frac{\left\langle\hat{a}^{\dagger} \hat{a}^{\dagger} \hat{a} \hat{a}\right\rangle}{\left\langle\hat{a}^{\dagger} \hat{a}\right\rangle^{2}}=1+\frac{(\Delta n)^{2}-\langle n\rangle}{\langle n\rangle^{2}} \quad \Rightarrow \quad g^{(2)}(0) \geq 1-\frac{1}{\langle n\rangle} .
$$

The intensity correlation function is measured with a Hanbury-Brown and Twiss (HBT) setup, that consists of a 50:50 beam splitter and photo detectors (APDs) on each output port (for detailed specifications of the HBT setup used in the experiments here see appendix B). Correlation of the arrival times of the photons on each detector 
and appropriate normalization yields the $g^{(2)}$ function [212]. For a true single photon source there will be no simultaneous detection events on both detectors, as the photon cannot be split and only take one of the two output paths of the beam splitters. The absence of correlation events at $\tau=0$ is called antibunching.

Note that for an ideal single emitter $g^{(2)}(0)=0$. Background fluorescence will lead to an increased value $g^{(2)}(0)>0$. The limit of 0.5 arises from a Fock state with 2 photons $|n=2\rangle$. This limit is strictly speaking only valid for two identical emitters. For two emitters with different emission rate and no (or very low background fluorescence) we will measure a value between 0 and 0.5 : $0<g^{(2)}(0)<0.5$. Thus, to strictly verify the presence of a single emitter, exact knowledge of the signal to noise ratio (SNR) is required. It limits the value of the $g^{(2)}$-function at zero delay to

$$
g^{(2)}(0)=1-\left(\frac{\mathrm{SNR}}{\mathrm{SNR}+1}\right)^{2} .
$$

Measuring the intensity correlation not only allows to discriminate the number of emitters (in case of only a few emitters), but also gives some insights into the dynamics of the emitter. The retrievable information strongly depends on the model we assume for the emitter. We will use here a simplified effective 3-level model for the NV center, that consists of a ground level $|1\rangle$, an excited state $|2\rangle$ and an intermediate level $|3\rangle$. Using more levels in principle is possible. However, for more levels the deduction of the transition rates between the levels from the $g^{(2)}$-measurements is often ambiguous. Therefore there is only little gain in using more levels.

In Fig. 2.6 already a simplified 5-level scheme for the NV center was introduced. Because excitation at $532 \mathrm{~nm}$ will lead to spin-polarization into the magnetic $m_{s}=$ 0 sublevel, we can neglect the $m_{s}= \pm 1$ sublevels in the ground and excited state. However, we will have to take into account additionally switching between the two charge states of the NV center. As simplification we will include this switching into the intermediate level as illustrated in Fig. 3.6. Except for the transition from the ground state to the intermediate level, we allow for all possible transition with rates $k_{i j}$ from level $i$ to level $j$, as shown in Fig. 3.6, too. Since the switching between the two charge states is power depend, so are the rates $k_{23}$ and $k_{32}$. The excitation rate $k_{12}$ naturally also dependents on the power of the excitation laser. Note that we use here non-resonant excitation into a pump-band followed by a fast relaxation into the excited state. The fast relaxation allows to omit the pump-state and to describe the whole process simply by the rate $k_{12}$.

The population dynamics is described by the following rate equations:

$$
\frac{d}{d t}\left(\begin{array}{l}
\rho_{1} \\
\rho_{2} \\
\rho_{3}
\end{array}\right)=\left(\begin{array}{ccc}
-k_{12} & k_{21} & k_{31} \\
k_{12} & -\left(k_{21}+k_{23}\right) & k_{32} \\
0 & k_{23} & -\left(k_{31}+k_{32}\right)
\end{array}\right)\left(\begin{array}{c}
\rho_{1} \\
\rho_{2} \\
\rho_{3}
\end{array}\right),
$$

where $\rho_{i}$ is the population of level $|i\rangle$. They need to fulfill $\rho_{1}+\rho_{2}+\rho_{3}=1$. The intensity correlation $g^{(2)}(\tau)$ can be interpreted as the conditional probability of measuring a 

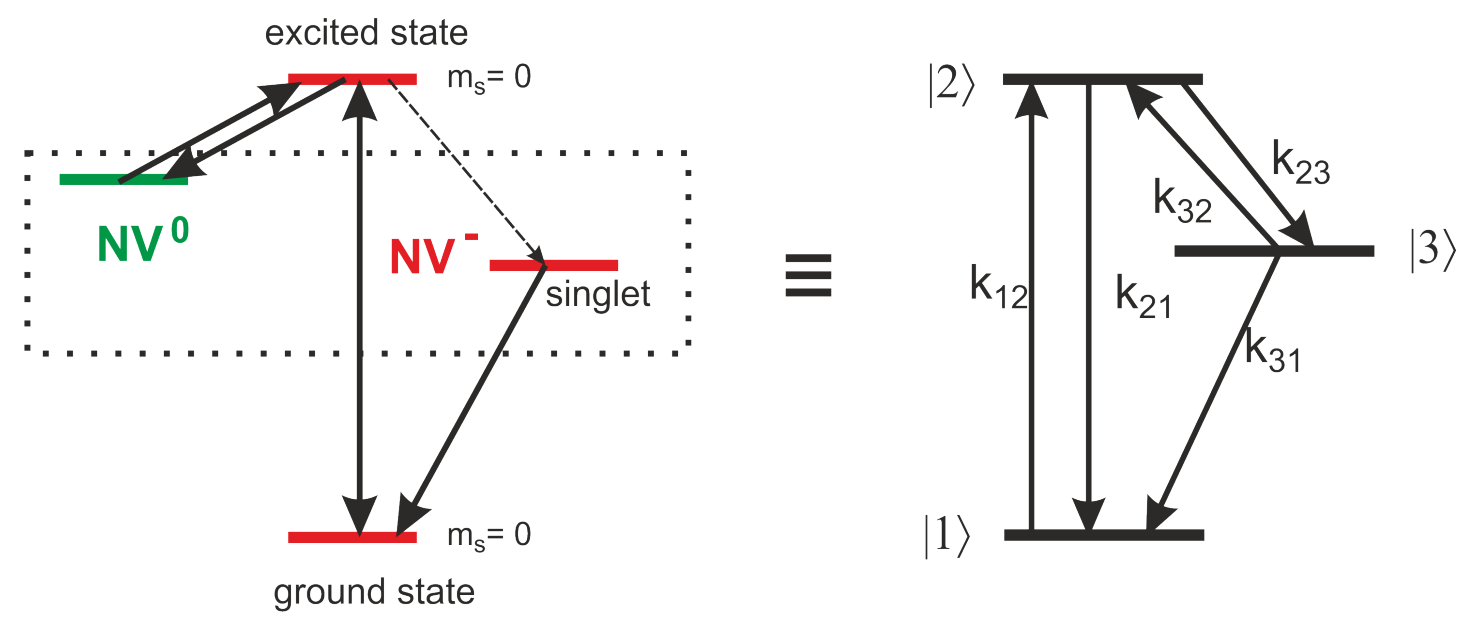

Figure 3.6: Switching to the neutral charge state and the intermediate singlet levels are summarized into one intermediate sublevel leading to an effective 3-level model. The parameters of this model, i.e. the transition rates between the levels, can be deduced from measurements of the intensity correlation function. Note that, due to spin polarization it is sufficient to only consider the $m_{s}=0$ sublevels.

photon at a time $t+\tau$ on the condition of having measured a photon at time $t$, normalized on the probability of having measured a photon at time $t$ :

$$
g^{(2)}(\tau)=\frac{P(t+\tau \mid t)}{P(t)}=\frac{\rho_{2}(\tau)}{\rho_{2}(\infty)} .
$$

We set the time of the emission of the first photon as $t=0$. The $g^{(2)}$-function then corresponds to the ratio of the population of the excited state at time $\tau$ normalized to its equilibrium population reached when $t \rightarrow \infty$ assuming the system initially at time $t=0$ to be in the ground state, which must be the case after emission of the first photon. In a more rigorous manner, this can be derived using the quantum regression theorem, which states that the expectation value of the two-time correlation function $\left\langle O\left(t^{\prime}\right) O(t)\right\rangle$ follows the same time evolution as the single time expectation value $\langle O(t)\rangle$. Using the fact that the photon number is proportional to the excited state population and that the $g^{(2)}$ function is normalized leads then directly to Eq. 3.5 with the appropriate initial condition $\left(\rho_{1}, \rho_{2}, \rho_{3}\right)(t=0)=(1,0,0)$. Solving Eq. 3.4 with this initial condition we find:

$$
g^{(2)}(\tau)=1-(1+a) e^{-|\tau| / \tau_{1}}+a e^{-|\tau| / \tau_{2}},
$$

where

$$
\begin{aligned}
\tau_{1,2} & =2 /\left(A \pm \sqrt{A^{2}-4 B}\right) \\
A & =k_{12}+k_{21}+k_{23}+k_{31}+k_{32} \\
B & =k_{12} k_{23}+k_{12} k_{31}+k_{12} k_{32}+k_{21} k_{31}+k_{21} k_{32}+k_{23} k_{31}
\end{aligned}
$$




$$
a=\frac{1-\tau_{2}\left(k_{31}+k_{32}\right)}{\left(k_{31}+k_{32}\right)\left(\tau_{2}-\tau_{1}\right)} .
$$

The power dependencies of the rates as motivated above are as follows:

$$
k_{12}=\sigma P, \quad k_{23}=k_{23}^{0}+\mu P, \quad k_{32}=\lambda P, \quad k_{21}=\text { const }, \quad k_{31}=\text { const },
$$

where $\sigma, \mu$ and $\lambda$ are parameters which need to be determined from measurements. The high- and low-power limits will aid to deduce some of the parameters. We find:

$$
\begin{aligned}
\tau_{1}^{0} & =\lim _{P \rightarrow 0} \tau_{1}=\frac{1}{k_{21}+k_{23}^{0}} \\
\tau_{2}^{0} & =\lim _{P \rightarrow 0} \tau_{2}=\frac{1}{k_{31}} \\
\tau_{1}^{\infty}=\lim _{P \rightarrow \infty} \tau_{1} & \propto \frac{1}{\sigma P} \\
\tau_{2}^{\infty}=\lim _{P \rightarrow \infty} \tau_{2} & \propto \frac{1}{(\mu+\lambda) P} \\
a^{\infty}=\lim _{P \rightarrow \infty} a & =\frac{\mu / \lambda}{1-(\mu+\lambda) / \sigma} \\
& \approx \frac{\mu}{\lambda} \text { for } \mu+\lambda \ll \sigma
\end{aligned}
$$

Note that the low power limit of $\tau_{1}$ corresponds to the excited state lifetime $\tau_{e}$.

$$
\tau_{e}=\lim _{P \rightarrow 0} \tau_{1}
$$

\subsubsection{Measurements of the intensity correlation function of several different single nitrogen-vacancy centers}

Four of the emitters shown in the scan above (Fig. 3.4) are single NV centers (\#5, \#31, \#38 and \#41). For their case we will now discuss measurements of the $g^{(2)}$-function and derivation of the internal rates. Two additional single NV centers in NDs on the same mirror but at different location and not shown in the scan, that have also been fully characterized, are also included in the discussion. First the saturation count rate and saturation power is determined as exemplarily shown for NV \#31 in Fig. 3.7. For this the emission rate as function of the excitation power is measured with single photon APDs (The count rates of the two APDs of the HBT are added). Saturation is observed according to the following equation:

$$
\mathrm{CR}=\mathrm{CR}_{\infty} \times \frac{P}{P+P_{\mathrm{sat}}}+\sigma_{\mathrm{bg}} \times P,
$$

where $\mathrm{CR}$ denotes the measure count rate, $\mathrm{CR}_{\infty}$ is the saturation count rate and $P_{\text {sat }}$ the saturation power. The last term takes into account background fluorescence which 


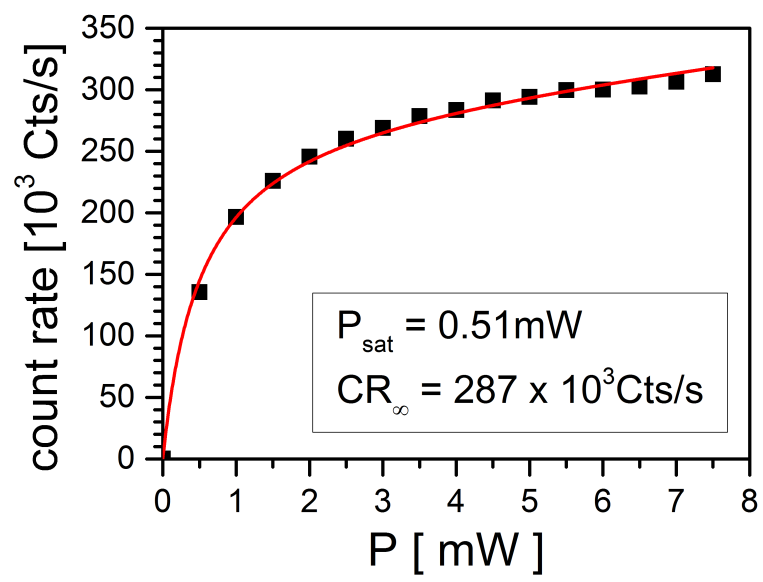

Figure 3.7: Saturation measurement for NV \#31: Count rate as detected on APDs in the spectral window $650-750 \mathrm{~nm}$ as function of the excitation power measured in front of the microscope objective. The measured data (black squares) are fitted with Eq. 3.11 (red curve) yielding $P_{\mathrm{sat}}=0.51 \mathrm{~mW}, \mathrm{CR}_{\infty}=287 \times 10^{3} \mathrm{Cts} / \mathrm{s}$ and $\sigma_{\mathrm{bg}}=6500 \mathrm{Counts} /(\mathrm{s} \mathrm{mW})$.

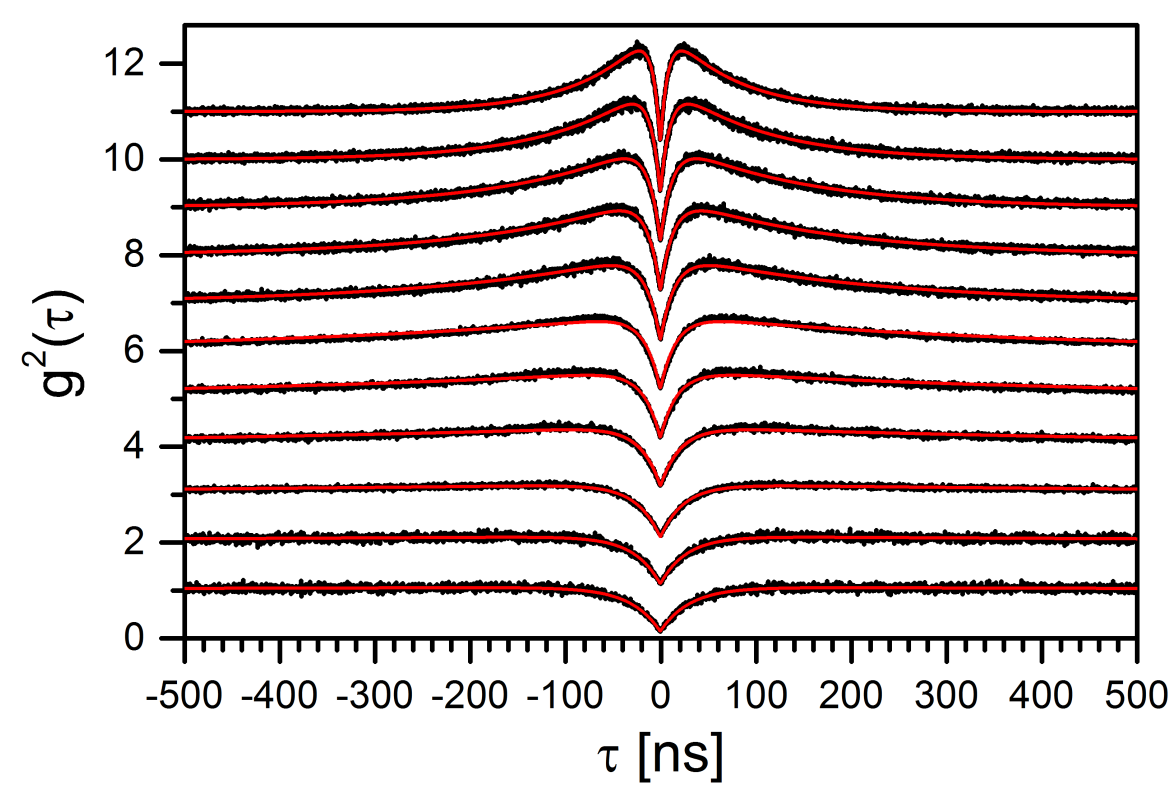

Figure 3.8: Black curve: Measured intensity correlation function for the NV \#31 at different excitation powers. The powers are from bottom to top: $0.29 \times, 0.59 \times, 1.0 \times, 2.0 \times, 2.9 \times, 3.9 \times$, $5.9 \times, 7.8 \times, 9.8 \times, 14 \times$ and $20 \times P_{\text {sat }}$. The curves are shifted vertically by 1 each for better visibility.

Red Curve: Fit of the measured data with $g^{(2)}(\tau)=1-(1+a) e^{-|\tau| / \tau_{1}}+a e^{-|\tau| / \tau_{2}}$. 
has a linear power-dependency. Typically a saturation count rate of several $10^{5} \mathrm{Cts} / \mathrm{s}$ is measured in the spectral window of $650-750 \mathrm{~nm}$. Compared to saturation count rates observed from NV centers in ND on a silicon substrate, the count rate observed here from NV centers on a mirror is roughly a factor 3-4 higher.

Next the intensity correlation is measured at different excitation power. The obtained results for NV \#31 are shown in Fig. 3.8. Beside the clear antibunching at $\tau=0$ we also observe for high excitation power an increased conditional probability of detecting a second photon at a time difference $\tau$ between approximately $20 \mathrm{~ns}$ and up to several hundred ns after the detecting the first photon manifested by a $g^{(2)}(\tau)>1$. This is a called bunching and is a clear signature, that at least one meta-stable state is involved in the internal dynamics. The normalized measured data is fitted according to the above derived expression (Eq. 3.6) to deduce the underlying rates. In Fig. 3.9 the obtained parameters $\tau_{1}, \tau_{2}$ and $a$ are displayed for six different single NV centers as function of the excitation power normalized to the measured saturation power of each center. Using the high- and low-power limits (Eqs. 3.9) and iterative fitting of $t_{1}$ and $t_{2}$ yields the underlying rates. They are summarized in table 3.1. Using the obtained rates the theoretical power dependence of $\tau_{1}, \tau_{2}$ and $a$ (Eqs. 3.7) can be calculated. They are also plotted in Fig. 3.9. For most cases the effective 3-level model well reproduces the results obtained from measurements of the intensity correlation function. However, there are few exceptions as for example the case of NV6 shown in Fig. 3.9, where the bunching persists even at low excitation powers $(a>0$ for $P \rightarrow 0)$.

The orders of magnitude of the derived parameters agree for all six NV centers, that are shown. However, due to the inherent variation of local environment for each of the NDs, the exact values slightly differ. We observe values for the excited state lifetime $\left[\tau_{e}=\lim _{P \rightarrow 0}\left(\tau_{1}\right)\right]$ of approximately $\tau_{e}=30 \mathrm{~ns}$. As expected, they are longer

\begin{tabular}{|ll|c|c|c|c|c|c|}
\hline \hline & & $\# 5$ & $\# 31$ & $\# 38$ & $\# 41$ & NV 5 & NV6 \\
\hline$P_{\text {sat }}$ & {$[\mathrm{mW}]$} & 0.59 & 0.51 & 0.47 & 0.46 & 0.76 & 0.93 \\
$\mathrm{CR}_{\infty}$ & {$\left[10^{5} \mathrm{Cts} / \mathrm{s}\right]$} & 142 & 287 & 282 & 290 & 240 & 251 \\
$k_{21}$ & {$[\mathrm{MHz}]$} & 32.2 & 31.6 & 29.3 & 36.8 & 42.1 & 33.6 \\
$\sigma$ & {$\left[\mathrm{MHz} \times P_{\text {sat }}^{-1}\right]$} & 6.8 & 5.8 & 4.5 & 7.1 & 8.2 & 10 \\
$\mu$ & {$\left[\mathrm{MHz} \times P_{\text {sat }}^{-1}\right]$} & 0.71 & 0.55 & 1.2 & 0.83 & 2.9 & 1.6 \\
$k_{23}^{0}$ & {$[\mathrm{MHz}]$} & 0 & 1.4 & 2.3 & 0.2 & 6.4 & 0.1 \\
$k_{31}$ & {$[\mathrm{MHz}]$} & 0.4 & 0.81 & 0.8 & 1.1 & 0.83 & 2.5 \\
$\lambda$ & {$\left[\mathrm{MHz} \times P_{\text {sat }}^{-1}\right]$} & 0.23 & 0.14 & 0.37 & 0.19 & 0.95 & 0.18 \\
\hline \hline
\end{tabular}

Table 3.1: Saturation power, saturation count rate and transition rates describing the population dynamics of the effective 3 level model for six different single NV centers in a ND on a mirror. The factors $\sigma, \mu$ and $\lambda$ are the proportionality factors in the power dependent rates according to Eq. 3.8. 

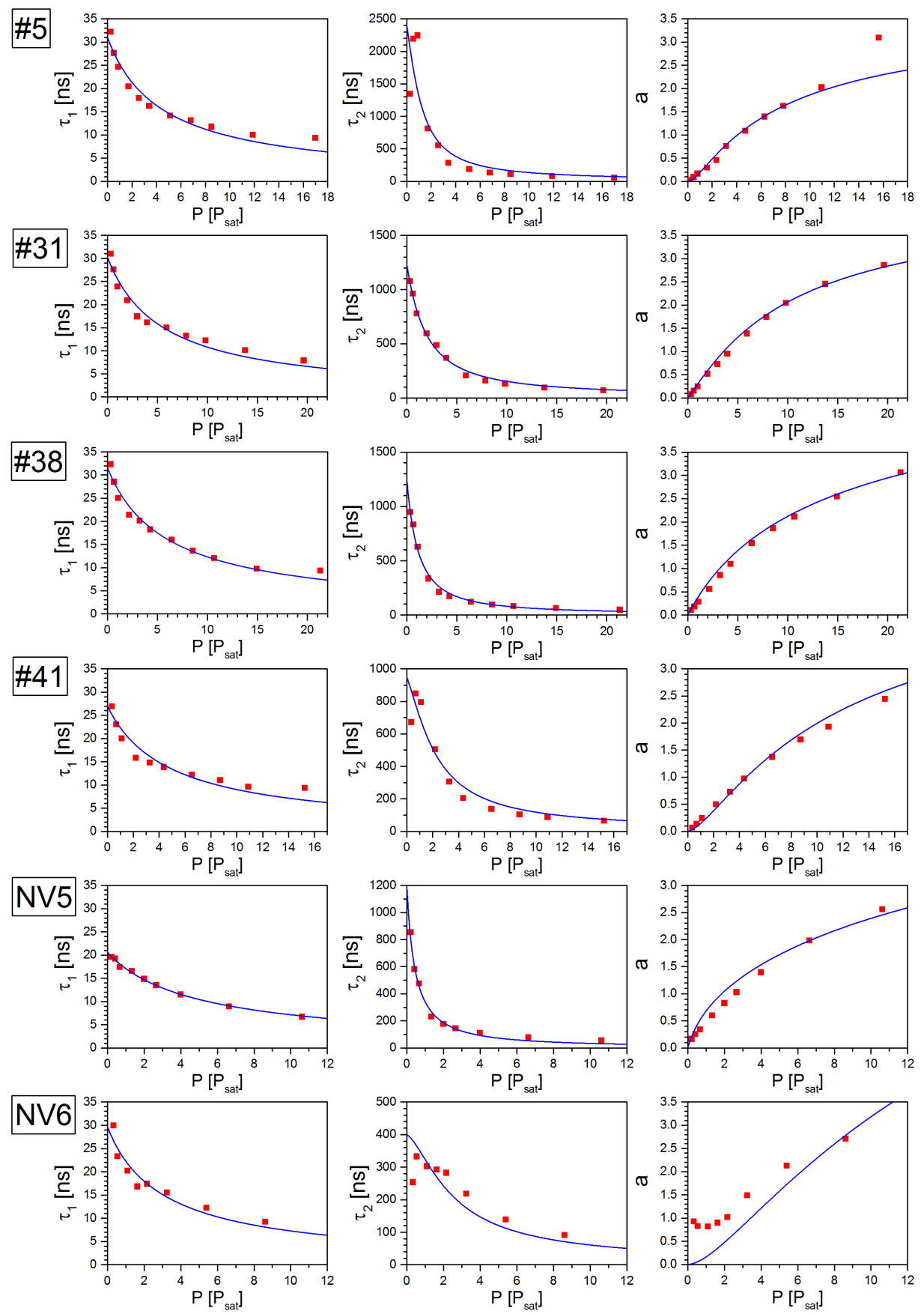

Figure 3.9: Red Squares: Obtained fit parameters $\tau_{1}, \tau_{2}$ and $a$ for 6 different single NV centers as function of the excitation power $P$ in units of the saturation power $P_{\text {sat }}$ of each NV center. Blue line: Power dependence of $\tau_{1}, \tau_{2}$ and $a$ according to the effective 3 level model, where the rates have been deduced from the high-power and low-power limits and fitting of $\tau_{1}$ and $\tau_{2}$. 
compared to a NV center in bulk diamond with $\tau_{e_{\text {bulk }}}=12.9 \mathrm{~ns}$ because these NV centers are hosted in a ND that is smaller than the wavelength and thus can be assumed to be effectively in air, where the density of states for the electric field is a factor of $n_{\text {diamond }}=2.42$, the refractive index of diamond, smaller than in the bulk case. An exception is NV 5 , for which we observe a shorter excited state lifetime of $\tau_{e} \approx 20 \mathrm{~ns}$. The reduced lifetime is mostly attributed to an increased decay rate $k_{23}^{0}$ to the singlet levels. NV 5 is an ideal example how the strain present in the ND leads to non-spinconserving dynamics contrary to an ideal NV center: By off-resonant excitation at $532 \mathrm{~nm}$ we would expect spin polarization into the $m_{s}=0$ sublevel. However, the probability from the $m_{s}=0$ excited state to decay to the singlet level is low and does not agree with obtained value for $k_{23}^{0}$. We can conclude that NV 5 must experience a large strain, that leads to a spin-mixing within the excited state. The fact that the excited state lifetime for the $m_{s}= \pm 1$ sublevel is faster compared to the $m_{s}=0$ level is thus consistent with the shorter measured lifetime of NV 5 and confirms the conclusion of strain-induced spin-mixing. Even though we have assumed a simplified 3-level model to analyze the measured $g^{(2)}$-functions, this example shows, that from this analysis we were able to gain significant insights on the dynamics of the NV center.

\subsection{Fluorescence spectra of single nitrogen-vacancy cen- ters}

\subsubsection{Spectra at room temperature}

As mentioned before, analyzing the fluorescence spectra allows gaining knowledge of the vibronic properties. For the case of NVs hosted in NDs, the spectrum from NV to NV will slightly differ. To quantify how strong these fluctuations are, we will here summarize the spectra from several different single NV centers. Figure 3.10 shows the fluorescence spectra from eight different single NV centers, where the first six stem from the centers, whose internal dynamics has just been discussed in the last section. For the details on the spectrometer employed see appendix B. The spectrum that differs most from the others corresponds to NV 5, where analyzing the internal population dynamics has revealed a spin-mixing within the excited state due to a large strain. It's not surprising that this large strain within the ND will also alter the coupling of the NV center to the local lattice vibrations. Thus for NV 5 the different emission spectrum is consistent with the deduced internal population dynamics. For the other shown NV centers there are only some slight variations. Each NV center sees a slightly different environment. As described in section 2.4.2, fitting the spectrum allows to derive the parameters to describe the NV center by a Huang-Rhys model. Each of the spectra is thus fitted with 8 Lorentzian lines. The obtained fit parameters are listed in table 3.2.

We can use the obtained Lorentzian linewidths to derive the parameters for the NV center employed in the model to describe the coupling to a microcavity, as has been discussed before in Sec. 2.4. To recall, we have modeled the NV center assuming 

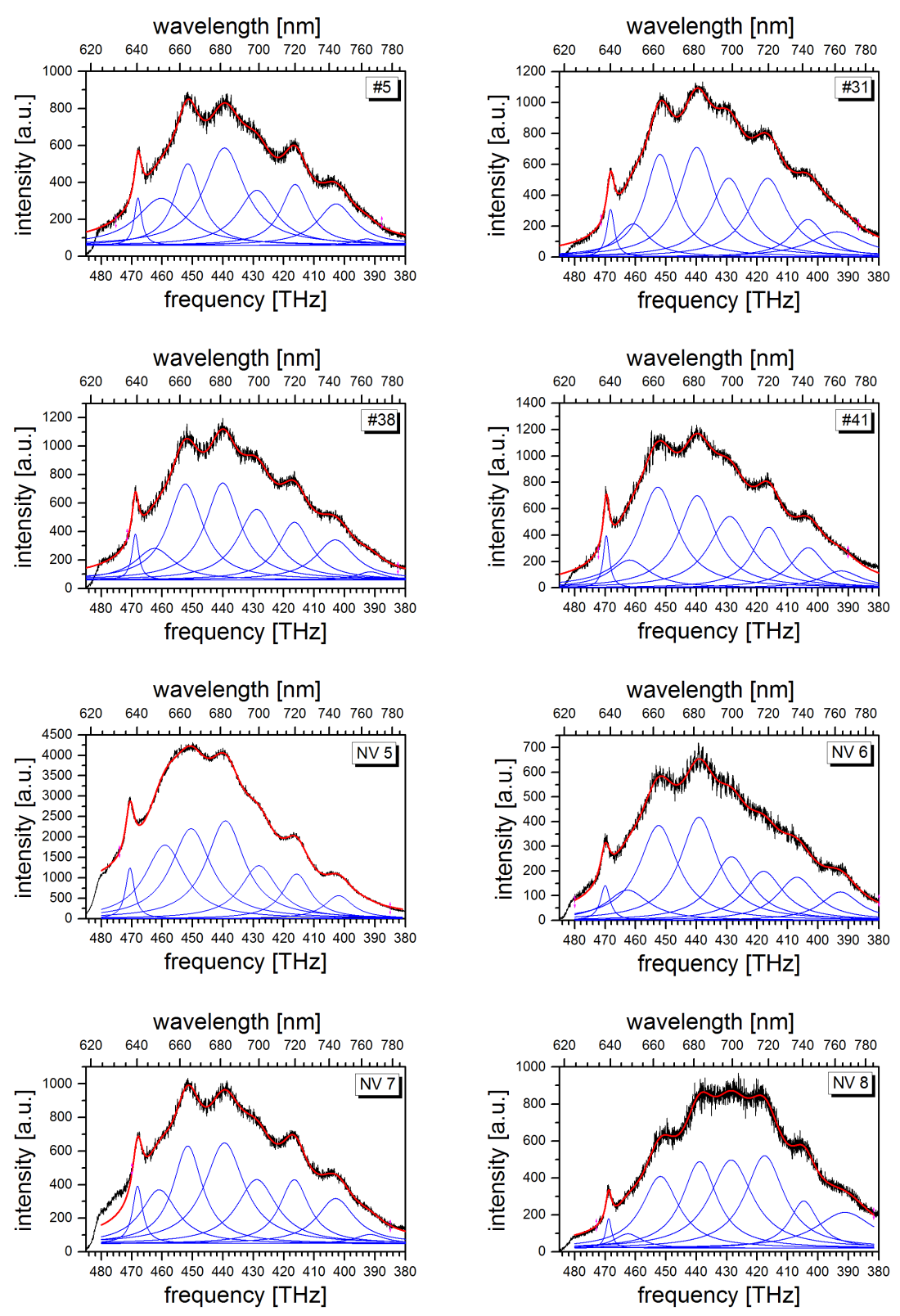

Figure 3.10: Fluorescence spectra of eight different NDs containing a single NV center: the first 6 correspond to the same NV centers whose internal dynamics has been discussed above. The spectra have been fitted with Lorentzian lines. The obtained parameters are listed in table 3.2. NV 8 is within a ND spin-coated onto a silicon substrate. The other seven are within a ND on a mirror. 


\begin{tabular}{|c|c|c|c|c|c|c|c|c|c|c|}
\hline & & & $\# 5$ & \#31 & \#38 & $\# 41$ & NV 5 & NV 6 & NV 7 & NV 8 \\
\hline \multirow{4}{*}{$\underline{1}$} & $\nu_{c}$ & {$[\mathrm{THz}]$} & 67.9 & 468.2 & 468.8 & 469.6 & 470.6 & 470 & 468.1 & 468.8 \\
\hline & $\lambda_{c}$ & {$[\mathrm{~nm}]$} & 640.5 & 640.1 & 639.3 & 638.2 & 636.9 & 637.7 & 40.3 & 39.2 \\
\hline & $\delta \nu$ & {$[\mathrm{THz}]$} & 2.9 & .0 & 2.7 & 2.4 & 3.8 & 3.5 & 4.0 & 2.3 \\
\hline & $A_{\mathrm{rel}}$ & {$[\%]$} & .4 & 2.1 & 2.1 & 0 & 2.0 & 3.0 & 3.6 & 1.0 \\
\hline \multirow{4}{*}{$\begin{array}{l}n \\
-1 \\
-\end{array}$} & $\nu_{c}$ & {$[\mathrm{THz}]$} & 0.0 & 460.7 & 462.5 & 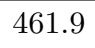 & 459.1 & 462.6 & 461.0 & 462.5 \\
\hline & $\delta \nu$ & {$[\mathrm{THz}]$} & .6 & 12.8 & 15.6 & 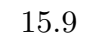 & 16.0 & 17.0 & 14.4 & 11.1 \\
\hline & $A_{\mathrm{rel}}$ & {$[\%]$} & 16.2 & 6.2 & 5 & 0 & 20.1 & 7.8 & 2.4 & 2.4 \\
\hline & $\Delta E$ & {$[\mathrm{meV}]$} & 31.7 & 31.0 & 26.1 & 31.7 & 45.1 & 30.4 & 29.3 & 29.3 \\
\hline \multirow{4}{*}{$\begin{array}{l}n \\
\text { क } \\
\text { iv }\end{array}$} & $\nu_{c}$ & {$[\mathrm{THz}]$} & 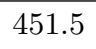 & 451.9 & 452.4 & 452.6 & 450.5 & 452.4 & 51.6 & 451.8 \\
\hline & $\delta \nu$ & {$[\mathrm{THz}]$} & 10.5 & 12.0 & 13.6 & 15.4 & 16.0 & 15.4 & 11.8 & 14.6 \\
\hline & $A_{\mathrm{rel}}$ & {$[\%]$} & 15.1 & 18.2 & 22.4 & 24.9 & 24.6 & 22.3 & 18.3 & 15.5 \\
\hline & $\Delta I$ & {$[\mathrm{meV}]$} & 67.8 & 67.2 & 68.0 & 70.2 & 80.7 & 72.7 & 68.1 & 73.5 \\
\hline \multirow{4}{*}{$\begin{array}{l}n \\
\theta \\
\infty\end{array}$} & $\nu_{c}$ & {$[\mathrm{THz}]$} & 439.5 & 439.9 & 440.0 & 439.7 & 439.2 & 439.1 & 439.5 & 438.9 \\
\hline & $\delta \nu$ & {$[\mathrm{THz}]$} & 16.2 & 14.4 & 13.8 & 15.0 & 15.3 & 15.3 & 15.8 & 13.8 \\
\hline & $A_{\mathrm{rel}}$ & {$[\%]$} & 27.7 & 23.2 & 23.1 & 21.9 & 25.5 & 24.0 & 25.2 & 17.6 \\
\hline & $\Delta E$ & {$[\mathrm{meV}]$} & 117.6 & 117.1 & 118.8 & 123.5 & 127.4 & 127.4 & 118.1 & 126.8 \\
\hline \multirow{4}{*}{$\begin{array}{l}n \\
\text { क } \\
\text { मे }\end{array}$} & $\nu_{c}$ & {$[\mathrm{THz}]$} & 428.8 & 429.3 & 428.9 & 429.0 & 428.2 & 428.4 & 428.9 & 428.5 \\
\hline & $\delta \nu$ & {$[\mathrm{THz}]$} & 15.1 & 14.7 & 15.0 & 16.5 & 14.7 & 15.5 & 15.9 & 17.1 \\
\hline & $A_{\mathrm{rel}}$ & {$[\%]$} & 14.7 & 17.0 & 18.3 & 18.7 & 13.3 & 15.0 & 16.2 & 22.3 \\
\hline & $\Delta E$ & {$[\mathrm{meV}]$} & 161.6 & 160.7 & 164.8 & 167.8 & 172.8 & 171.7 & 162.0 & 169.7 \\
\hline \multirow{4}{*}{$\begin{array}{l}0 \\
\text { क } \\
\text { D. }\end{array}$} & $\nu_{c}$ & {$[\mathrm{THz}]$} & 416.2 & 416.6 & 416.5 & 416.2 & 415.8 & 417.9 & 416.5 & 417.5 \\
\hline & $\delta \nu$ & {$[\mathrm{THz}]$} & & 15 & 13.2 & 12.7 & 11.3 & 15.7 & 11.7 & 15.5 \\
\hline & $A_{\mathrm{rel}}$ & {$[\%]$} & 12.0 & 17.8 & 13.1 & 12.2 & 8.6 & 11.8 & 11.9 & 21.1 \\
\hline & $\Delta E$ & {$[\mathrm{meV}]$} & 213.6 & 213.4 & 216.3 & 220.7 & 224.1 & 215.3 & 213.0 & 215.2 \\
\hline \multirow{4}{*}{$\begin{array}{l}n \\
0 \\
0\end{array}$} & $\nu_{c}$ & {$[\mathrm{THz}]$} & 4 & 403.3 & 403.1 & 403.2 & 40 & 406.9 & 402.9 & 404.7 \\
\hline & $\delta \nu$ & {$[\mathrm{THz}]$} & 14.7 & 13.0 & 15.7 & 13.7 & 11.4 & 15.7 & 15.0 & 11.3 \\
\hline & $A_{\mathrm{rel}}$ & {$[\%]$} & 10.8 & 7.2 & 10.9 & 88 & 4.5 & 10.3 & 10.7 & 7.9 \\
\hline & $\Delta E$ & {$[\mathrm{meV}]$} & 269.2 & 268.2 & 271.7 & 274.4 & 281.1 & 260.5 & 269.3 & 268.4 \\
\hline \multirow{4}{*}{$\begin{array}{l}n \\
\text { n } \\
r\end{array}$} & $\nu_{c}$ & {$[\mathrm{THz}]$} & 392.7 & 393.8 & 391.1 & 392.4 & 393.8 & 392.6 & 391.7 & 391.1 \\
\hline & $\delta \nu$ & {$[\mathrm{THz}]$} & 9.9 & 21.9 & 10.7 & 16.2 & 12 & 15.5 & 11.7 & 23.2 \\
\hline & $A_{\mathrm{rel}}$ & {$[\%]$} & 1.1 & 8.1 & 1.5 & 4.4 & 0.5 & 6.7 & 1.7 & 12.3 \\
\hline & $\Delta E$ & {$[\mathrm{meV}]$} & 310.7 & 307.7 & 319.6 & 319.2 & 314.8 & 319.6 & 315.8 & 324.4 \\
\hline
\end{tabular}

Table 3.2: Parameters obtained from fitting the spectra of different single NV centers as shown in Fig. 3.10 each with 8 Lorentzian lines. $\nu_{c}$ denotes the central frequency, $\lambda_{c}$ the corresponding wavelength (only shown for the ZPL), $\delta \nu$ the FWHM and $\Delta E$ the energy difference of the higher vibrational levels relative to the vibrational ground state $\left(\Delta E=\hbar\left[\left(\nu_{c}(\mathrm{ZPL})-\nu_{c}\right]\right) . A_{\text {rel }}\right.$ is the relative area of each Lorentzian line compared to the sum of the area of all eight lines. 
$n$ vibrational ground states and one excited state. The energies of the levels for the ground state are

$$
E_{i} / \hbar=2 \pi\left(\nu_{c i}-\nu_{c 0}\right) \quad i=0, \ldots, 7
$$

and for the excited state

$$
E_{e} / \hbar=2 \pi \nu_{c 0} .
$$

The strength of the spontaneous decay is denoted by $\gamma_{i}$ and is determined by:

$$
\gamma_{i}=A_{\text {rel }} \cdot \gamma_{\text {tot }} \quad i=0, \ldots, 7 \quad \text { where } A_{\text {rel }} \equiv \frac{A_{i}}{\sum_{i} A_{i}} .
$$

$A_{i}$ denotes the area under each of the eight Lorentzian lines the spectrum has been fitted with. The total transition rate $\gamma_{\text {tot }}$ has already been deduced from the measurements of the $g^{(2)}$-function (cf. last Sec. 3.2.2) as: $\gamma_{\text {tot }}=k_{21}$.

We further take into account two broadening effects. First, pure dephasing with rate $\gamma^{\star}$, which is assumed to be the same for all eight transition and second, broadening due to fast relaxation of the higher vibronic ground states with rates $\gamma_{i, i-1}$, that only affect the transitions forming the PSB. They are determined from the obtained fit parameters by:

$$
\begin{aligned}
\gamma^{*} & =2 \pi \cdot \delta \nu_{0} \\
\gamma^{*}+\gamma_{i, i-1} & =2 \pi \cdot \delta \nu_{i} \quad i=1, \ldots, 7
\end{aligned}
$$

We see that the fitted lines are not spaced equally and thus a Huang-Rhys model with a single vibrational mode is not accurate. Though, the obtained values agree well with some of the vibronic levels calculated by Abtew et al. [133]. They found the following energies for the first vibronic levels: $36.7 \mathrm{meV}, 71.7 \mathrm{meV}, 103.8 \mathrm{meV}, 114.8 \mathrm{meV}$, $143.7 \mathrm{meV}, 166.1 \mathrm{meV}, 183.7 \mathrm{meV}$ and $199.6 \mathrm{meV}$. They are also found in measurements: via two-dimensional electronic spectroscopy Huxter et al. obtained for the energies of the vibrational levels the values: $23.0 \mathrm{meV}, 33.1 \mathrm{meV}, 43.5 \mathrm{meV}, 59.9 \mathrm{meV}$, $74.2 \mathrm{meV}, 86.6 \mathrm{meV}, 100.3 \mathrm{meV}, 146.1 \mathrm{meV}, 169.1 \mathrm{meV}, 199.4 \mathrm{meV}$ and $233.8 \mathrm{meV}$. Averaging over the values for all 8 centers we have here obtained for the 7 vibrational energies: $31.8 \mathrm{meV}, 67.3 \mathrm{meV}, 122.1 \mathrm{meV}, 166.3 \mathrm{meV}, 216.5 \mathrm{meV}, 270.4 \mathrm{meV}$ and $316.5 \mathrm{meV}$. These are less vibronic levels compared to the predicted/measured values. The fit of the spectrum is not unique: because the bands strongly overlap the recognition of more levels within the spectrum is obscured.

The strain within the NDs manifests itself also in the varying position of the ZPL. For the eight shown single NVs the range of the ZPL position is $\Delta \lambda_{\mathrm{ZPL}}=3.6 \mathrm{~nm}$ in wavelength corresponding to $\Delta \nu_{\mathrm{ZPL}}=2.7 \mathrm{THz}$ in frequency. As we have identified before from analyzing the $g^{(2)}$-measurements, NV 5 experiences a large strain. This also is reflected in the emission spectrum, which significantly differs from the other 7 shown spectra. It is not surprising that the large strain in the local environment of NV 5 modifies the local vibrations and the coupling of the NV center to them. 


\subsubsection{Spectra at cryogenic temperatures}

When the NV center is cooled to cryogenic temperatures, the linewidths of the ZPL become narrower because phonon scattering is reduced. For an ideal NV center one can reach a lifetime limited linewidth $(\delta \nu=13 \mathrm{MHz})$ of the ZPL [14]. However, as discussed before, in most HPHT NDs the linewidth is limited by spectral diffusion. The measurements shown in this section are from the same type of NDs spin-coated onto a silicon substrate, which then is mounted in a continuous flow cryostat (Janis ST-500), that can reach temperatures down to $4.2 \mathrm{~K}$. All specified temperatures in the following are measured on the cold finger of this cryostat. Figure. 3.11(a) shows the spectrum of a single NV center at $\approx 10 \mathrm{~K}$. The PSB structure is only slightly altered compared to the room temperature spectrum. However, the ZPL becomes much more prominent, as the emission is into a narrower line.

The ZPL has a Gaussian line shape, which indicates inhomogeneous broadening, with a FWHM of $\delta \nu=580 \mathrm{GHz}(\delta \lambda=0.79 \mathrm{~nm})$. A linewidth of this order of magnitude is typically measured for NV centers within the NDs provided by Microdiamant. Figure 3.11(b) and (c) show the spectra of two further NDs containing 2 or 4 NV centers. Fitting the linewidth yields values in the range of $\delta \nu=190-550 \mathrm{GHz}$ $(\delta \lambda=0.26-0.73 \mathrm{~nm})$ for all a $6 \mathrm{NV}$ centers. Once more, the influence of strain on the ZPL position is visible. Even within the same ND the strain that the different NV experience varies such that the different ZPLs are resolved at cryogenic temperatures.

The limitation due to spectral diffusion can also be seen, when measuring the linewidth as function of temperature. An example of a ND with $4 \mathrm{NV}$ centers is shown in Fig. 3.12 containing the spectra of the 4 ZPLs at different temperatures. They are fitted with 4 Gaussian lines to determine the width. The obtained values are displayed in Fig. 3.13. At temperatures below $100 \mathrm{~K}$ they are constant, and only above they start to increase.

As the simulation have shown in Sect. 2.5.4, the pure dephasing $\gamma^{\star}$ needs to be reduced to values in the range $1-10 \mathrm{GHz}$, such that the cavity decay rate $\boldsymbol{\kappa}$ becomes the dominant loss channel an the Purcell regime is entered. In order to experience the large enhancement in emission the cavity needs to remain on resonance with the ZPL frequency of the NV center. Thus, jumps of the ZPL frequency due to spectral diffusion needs to be smaller than the cavity linewidth:

$$
\Delta \nu_{\text {spectraldiffusion }}<2 \pi \kappa \text {. }
$$

Hence, to investigate the Purcell regime, the HPHT NDs, that have been investigated here, are not well suited. Spectral diffusion linewidths smaller by at least two orders of magnitude are desirable. For the given cavity parameters used in this work we have a cavity decay rate $\boldsymbol{\kappa}=80 \mathrm{GHz}$. At the limit of feasible parameters the decay rate can reach $\boldsymbol{\kappa}=10 \mathrm{GHz}$.

As mentioned in the beginning of this chapter, fabrication of NDs hosting NV center with improved properties with respect to spectral diffusion and spin coherence is investigated. Most promising is to start from high quality CVD diamond. In most 
diamonds the ratio between substitutional nitrogen and NV centers is typically on the order of 10-20. Since substitutional nitrogen is one of the main sources for spectral diffusion this ratio is not very favorable. Thus the first step is to create additional vacancies via electron irradiation followed by annealing. If the density of vacancies is high enough, this allows then to transform most of the substitutional nitrogen into NV centers. Using this diamond as starting material, NDs can be fabricated via grinding by a BASD technique or fabricated by RIE. Depending on the final size of the NDs and the original concentration of NV centers a suitable yield of NDs that contain a single NV center should be realizable.

(a)

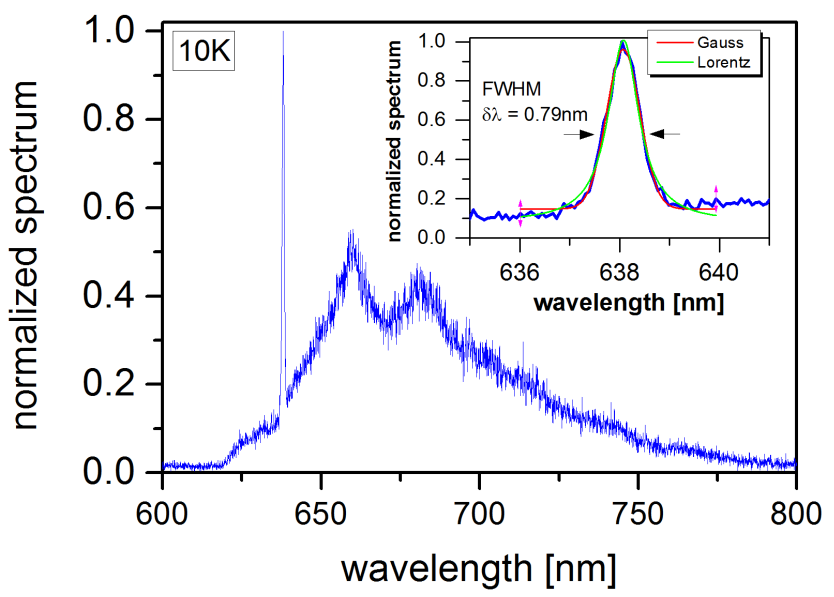

(b)

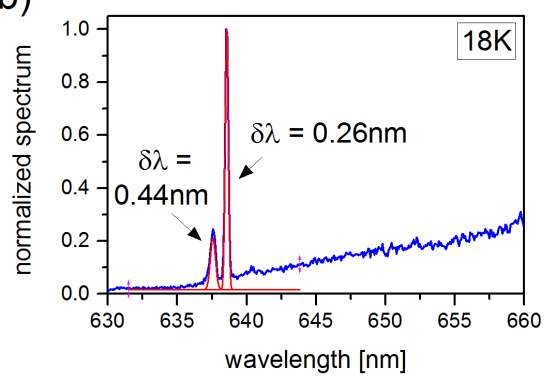

(c)

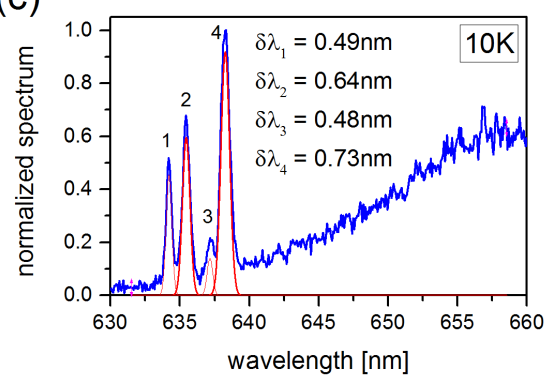

Figure 3.11: (a) Fluorescence spectrum of a single NV center at a temperature of $\approx 10 \mathrm{~K}$. The inset is a zoom into the ZPL and fits with either a Gaussian and Lorentzian line illustrate that the shape is clearly Gaussian with a FWHM of $\delta \lambda=0.79 \mathrm{~nm}$ which corresponds to a frequency width of $\delta \nu=580 \mathrm{GHz}$. (b) and (c) Fluorescence spectra of two further NDs containing two or four NV centers. The spectra are fitted with multiple Gaussians leading to widths of $\delta \lambda=$ $0.44 \mathrm{~nm}, 0.26 \mathrm{~nm}, 0.49 \mathrm{~nm}, 0.64 \mathrm{~nm}, 0.48 \mathrm{~nm}$ and $0.73 \mathrm{~nm}$ corresponding to frequency values of $\delta \nu=320 \mathrm{GHz}, 190 \mathrm{GHz}, 360 \mathrm{GHz}, 470 \mathrm{GHz}, 350 \mathrm{GHz}$ and $540 \mathrm{GHz}$ 

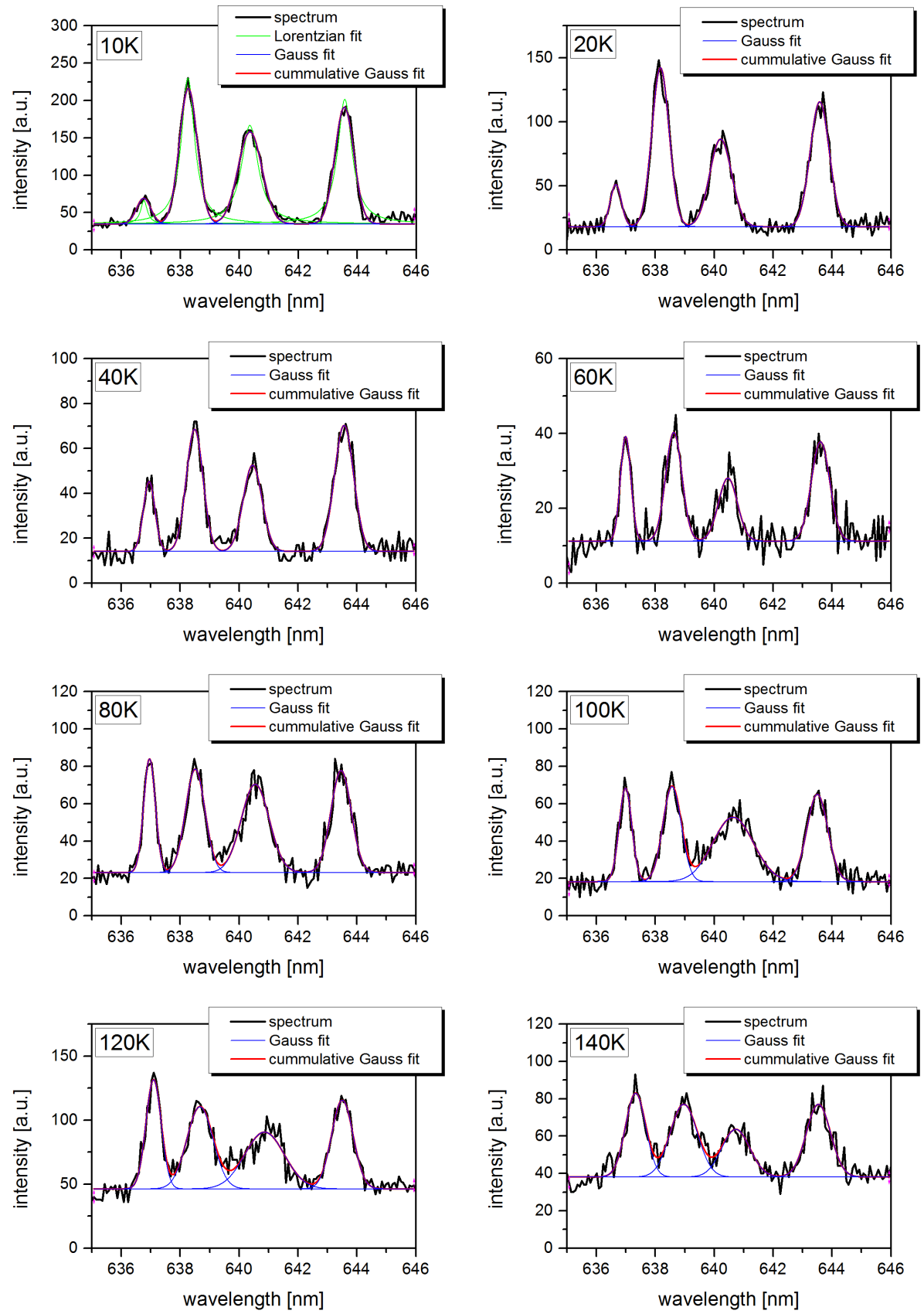

Figure 3.12: Fluorescence spectra of a ND containing $4 \mathrm{NV}$ centers recorded at different temperatures. Only the spectral region of the ZPLs is displayed. As shown in the spectrum at $10 \mathrm{~K}$ the line shape is clearly Gaussian. The widths of the $4 \mathrm{NV}$ center as function of temperature is plotted in Fig. 3.13. 


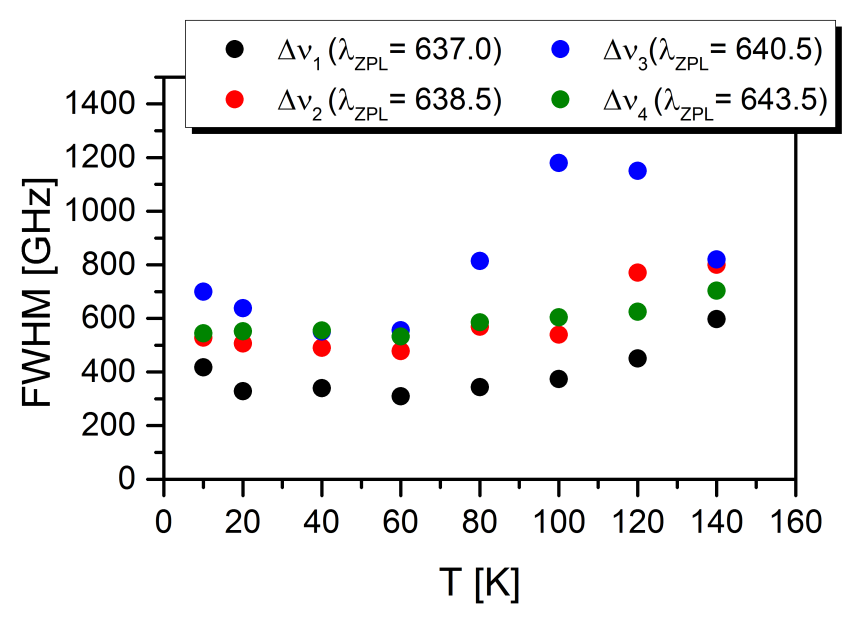

Figure 3.13: Linewidths of the 4 NVs whose spectra are shown in Fig. 3.12 as function of Temperature. Below $100 \mathrm{~K}$ they remain constant. Note that at higher temperature the fitted Gaussians for the third NV do not well reproduce the form of the spectrum due to overlapping with the ZPL of another NV center. 


\section{Chapter 4}

\section{Fiber resonators}

This chapter will introduce the details on the optical cavities employed in this work, i.e. fiber-based Fabry-Perot cavities. The basic characteristics of this cavity type have already been discussed in section 2.2. Here we focus on the special realization of such cavities, where one or both of the mirrors is directly fabricated on the facet of an optical fiber. In the first section the fabrication process of the fiber mirrors will be presented. The second part addresses technical issues such as background fluorescence of the fibers and cavity length stabilization.

\subsection{Fiber mirrors}

Fabricating a curved mirror on a fiber facet enables the realization of a fiber-based Fabry-Perot micro-cavity. Such cavities have several advantages, which make them attractive for use in quantum optics. The small foot-print enables the incorporation e.g. onto atom-chips $[213,214]$ or into ion traps [215]. Together with the achievable small radii of curvature, cavities with small mode volumes $\left(\approx 3 \mu \mathrm{m}^{3}\right)$ can be realized. Using highly reflective dielectric mirror coatings cavities with high finesse $>1.5 \times 10^{5}$ have been demonstrated [216]. In contrast to many other types of micro-cavities [71], they are easily tunable by controlling the cavity length via for instance a piezo-electric crystal. Additionally, the output of fiber cavities is automatically fiber-coupled.

Such fiber based microcavities have recently been employed to demonstrate coupling to molecules [217], quantum dots [187,188] and a mechanical oscillator consisting of a silicon nitride membrane [218]. Several different methods for the realization of such fiber tips have been developed. Laser machining and structuring by focused ion beam (FIB) have by now been established as the techniques of choice and both have been used within the scope of this work. 


\subsubsection{Structuring by laser machining}

Silica glass is a strong absorber for light with a wavelength of $10.6 \mu \mathrm{m}$, the emission wavelength of the $\mathrm{CO}_{2}$ laser. Therefore these lasers are used, beside many other industrial applications, for glass processing such as welding and engraving. The melting and re-crystallization of glass leads to very smooth surfaces and has also been utilized for the fabrication of silica based high Q whispering gallery mode resonators, for instance microspheres [219] and microtorroids [220]. Structuring fiber facets with the use of a $\mathrm{CO}_{2}$ laser has been developed in the group of Jakob Reichel, ENS Paris [221-223]. The laser machined fibers used in this work have been fabricated in collaboration with his group. The structuring is a combined process of evaporation and melting. A $\mathrm{CO}_{2}$ laser with power in the range $P=300 \mathrm{~mW}$ to $2 \mathrm{~W}$ is focused onto the facet for a short duration of time $\tau_{\mathrm{CO}_{2}}=4$ to $120 \mathrm{~ms}$. It is important that only a thin layer close to the surface is melted. Otherwise, due to surface tension, re-crystallization will yield a convex structure. For too short duration of the laser exposure the re-solidification process is too fast for the surface smoothing process to occur. This lower limit is not reached within the parameter range employed for fiber structuring $[222,223]$. The resulting profile from evaporation can approximately be modeled by calculating the surface ablation rate from the surface temperature profile. To gain the temperature profile, absorption, thermal conductivity and thermal diffusion need to be taken into account. This model well reproduces the structures fabricated on a glass substrate, when the thermal conductivity is treated as free fitting parameter [222,223]. On fiber facets, due to their smaller lateral dimensions, the predictions deviate from the effectively realized structures, especially the diameter is significantly ( $\approx$ by factor of 2 ) larger than expected from the model [222].

The resulting structure is imaged by white light interferometry. The result of such a measurement is show in Fig. 4.1(a). The profile along the two main axes of the slight elliptic shape are fitted by polynomial functions as shown in Fig 4.1(b). To determine the radius of curvature $R$ a quadratic function is used, which reproduces best the curvature of the profile close to the center of the imprint. It is worth noting that the radius of curvature is not constant over the complete profile. Fitting with a higher order polynomial yields the inflection points, where the shape changes from concave to convex. The effective diameter $D$ is defined as distance between the two inflection points and the effective height $h$ is the vertical distance from the lowest point to the inflection points. The complete depth $z_{\max }$ is the maximal vertical distance across the complete fiber facets and therefore the minimal geometric length possible for a cavity built with such a fiber and a plane mirror. The scattering losses due to the surface roughness are approximately given by [222]

$$
L_{\text {scatt. }} \approx\left(\frac{4 \pi \sigma_{\mathrm{RMS}}}{\lambda}\right)^{2} .
$$

The surface roughness has been measured with an AFM for only a few sample fibers resulting in $\sigma_{\mathrm{RMS}} \approx 0.2 \mathrm{~nm}$. This enables finesses greater $100,000[216,222]$. 
The resulting features vary significantly from shot to shot. By using the intensity of the emitted white light during exposure to the $\mathrm{CO}_{2}$ laser as feedback to control the exposure time, Petrak et al. showed that a much higher repeatability can be achieved [224]. They also have shown that this method is applicable to structure fibers, as the emitted white light is partly collected by the fiber and can be monitored at its other end.

(a)

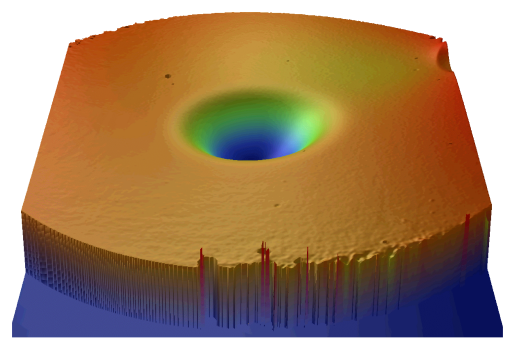

(b)

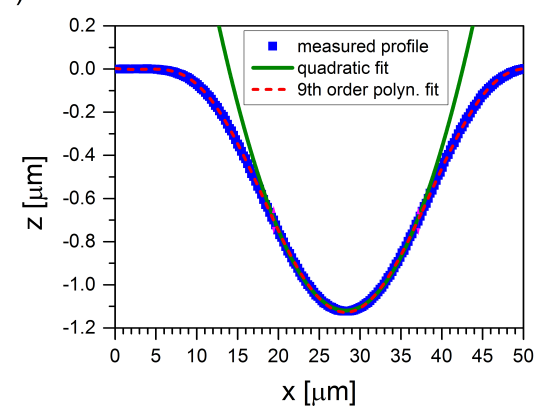

Figure 4.1: (a) 3D-Profile measured by white light interferometry of a imprint in a fiber facet produced with $\mathrm{CO}_{2}$ laser machining. (b) Cross-section of the profile along one of the main axis of the slight elliptic shape (ellipticity $\approx 0.3$ ) fitted with a higher order (9th) polynomial to determine the inflection points of the profile and in the center with a quadratic function to determine the radius of curvature.

For this work two kind of fibers have been structured with a $\mathrm{CO}_{2}$ laser:

- Oxford Electronics GI50-200CB CuBall graded index multi-mode (MM) fiber with a core of $50 \mu \mathrm{m}$ and cladding of $200 \mu \mathrm{m}$. The fiber has a copper based metallic coating.

- Fiberguide industries gold coated single-mode (SM) fiber with a mode field diameter of $4.3 \mu \mathrm{m}$, cladding of $125 \mu \mathrm{m}$ and numerical aperture of 0.12 .

\begin{tabular}{|c|c|c|c|c|c|c|c|}
\hline \hline Fiber type & $\begin{array}{c}P \\
{[\mathrm{~mW}]}\end{array}$ & $\begin{array}{c}w_{0} \\
{[\mu \mathrm{m}]}\end{array}$ & $\begin{array}{c}\tau_{\mathrm{CO}_{2}} \\
{[\mathrm{~ms}]}\end{array}$ & $\begin{array}{c}R \\
{[\mu \mathrm{m}]}\end{array}$ & $\begin{array}{c}D \\
{[\mu \mathrm{m}]}\end{array}$ & $\begin{array}{c}h_{\text {eff }} \\
{[\mu \mathrm{m}]}\end{array}$ & $\begin{array}{c}z_{\max } \\
{[\mu \mathrm{m}]}\end{array}$ \\
\hline Cubal MM & 0.54 & 27.8 & $22.5-70$ & $20-314$ & $12.2-28.6$ & $0.06-3.3$ & no data \\
Gold SM & 1.2 & 28 & $25-27$ & $66-107$ & $17.7-20.6$ & $0.36-0.77$ & $0.9-2.3$ \\
\hline \hline
\end{tabular}

Table 4.1: Fabrication parameters $P$ (laser power), $w_{0}$ (the beam waist of the focused laser on the fiber) and $\tau_{\mathrm{CO}_{2}}$ (exposure time) used and thereby resulting structures with radius of curvature $R$, effective diameter $D$, effective depth $h_{\text {eff }}$, complete depth $z_{\max }$ for the fibers machined with a $\mathrm{CO}_{2}$ lasers within the scope of this work. 
The fabrication parameters and resulting structures are summarized in table 4.1. No feedback-control has been employed during fabrication.

\subsubsection{Structuring by focused ion beam milling}

In an alternative approach fibers can also be structured by FIB milling. A beam of gallium ions $\left(\mathrm{Ga}^{+}\right)$is focused onto the fiber facet with a spot size of $\approx 100 \mathrm{~nm}$, that sputters off the silica from the surface. By scanning across the surface and varying the dwell time any given profile within the resolution of the spot size can be machined. As glass is an electrical isolator, building up of surface charges needs to be prevented. This charge neutralization is realized within the vacuum chamber with a electrical filament at a certain voltage, which floods the chamber with electrons, such that the charging of the sample by the ions is compensated.

To fabricate microcavities with small mode volumes Dolan et al. [225] also used FIB milling. They wrote arrays of spherical imprints into a plane substrate. After deposition of dielectric mirror coatings and using a second plane mirror the built planecurved Fabry-Perot cavities with mode volumes down to the order of femtoliter $\left(\mu m^{3}\right)$. Note that these cavities are not fiber-based and thus do not share the advantage of the output being automatically fiber-coupled and having a small foot-print. Common to both systems is the realization of easily tunable cavities with small mode volumes.

(a)

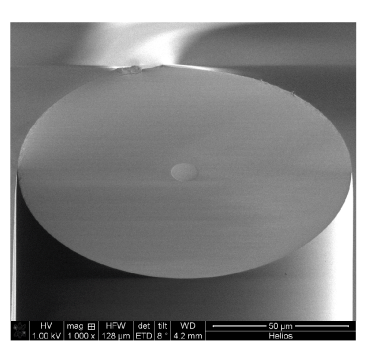

(b)

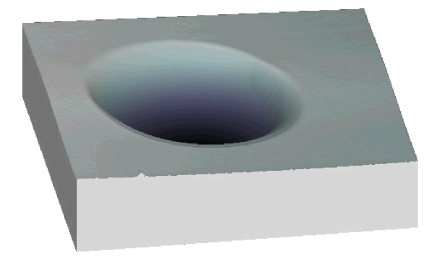

(c)

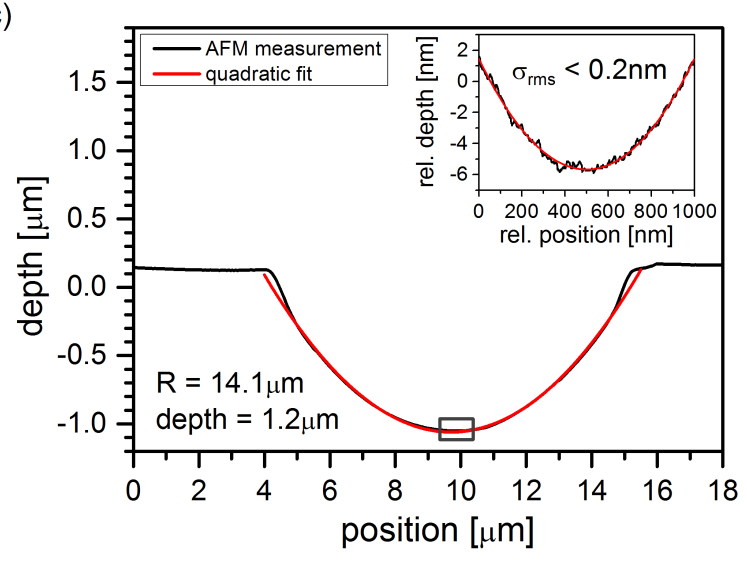

Figure 4.2: (a) SEM image of fiber facet with a spherical imprint written by FIB milling. (b) 3D image of the imprint as measured with an AFM (c) profile cut fitted with a quadratic polynomial yielding a radius of curvature of $R=14.2 \mu \mathrm{m}$. The depth of the structure is $h=1.2 \mu \mathrm{m}$ and the diameter is $D=11.1 \mu \mathrm{m}$. The inset shows the profile along its minimum revealing the very smooth surface roughness with $\sigma_{\text {rms }}<0.2 \mu \mathrm{m}$. 
Thanks to the fabricated array they have compared to fiber-based cavities the advantage of realizing simultaneously many cavities.

Within this work fibers have been structured with the following set of parameters: diameter $D=10 \mu \mathrm{m}$, radius of curvature $R=14.1 \mu \mathrm{m}$, depth $h=1.2 \mu \mathrm{m}$. One advantage of the FIB is the high passive repeatability i.e. the fabricated structure vary only very little from each other when using the same fabrication parameters. The system used is a "Helios Nanolab Dualbeam " from Fei, which, beside the ion column, also contains an electron microscope for imaging. Figure 4.2(a) exemplarily shows a scanning electron microscope (SEM) image of a machined fiber. The fabricated structure is measured with an AFM. The results are shown as 3D image in Fig. 4.2(b). To determine the radius of curvature, the cut through the center of the profile is fitted with a quadratic polynomial as displayed in Fig 4.2(c). The resulting features are very smooth and the surface roughness is only few Angström $\left(\sigma_{\text {rms }}<2 \AA\right)$, similar to the values reached with laser machining.

Four types of fiber have been structured with the FIB method within this work: the above described MM-fiber from Oxford electronics and gold coated SM-fiber from Fiberguide industries and furthermore the pure silica core SM-fibers S-460HP and S$630 \mathrm{HP}$ from Nufern (for details of the fibers see below table 4.2).

Whereas the small structures are promising to achieve a small mode volume and hence a large coupling rate, greater radii of curvatures and larger diameters are interesting for longer cavities, which are desirable e.g. for incorporation into ion traps, where the cavity needs to be large to prevent distortion of the trapping potential due to electric charging of glass fibers. For this purpose, the realization of larger imprints by

(a)

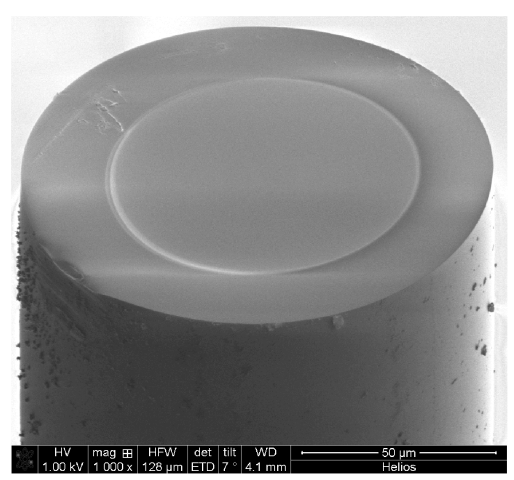

(b)

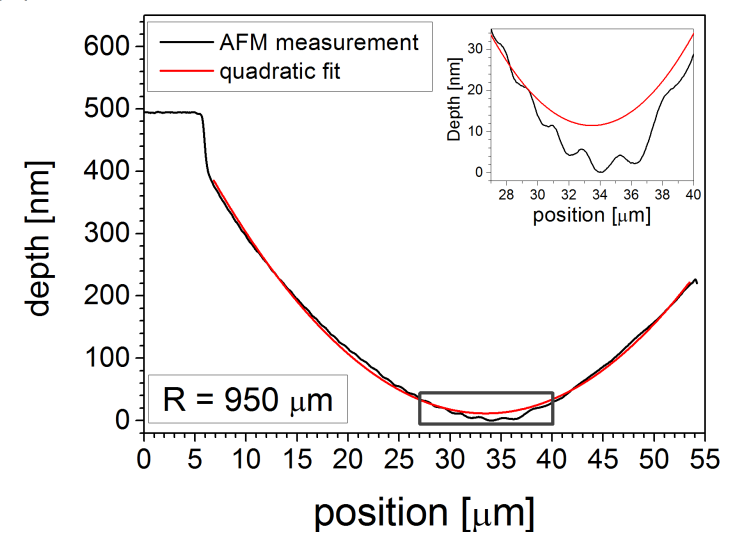

Figure 4.3: (a) SEM image of fiber facet with a spherical imprint written by FIB milling. The design diameter is $80 \mu \mathrm{m}$. Duration of the writing process was $38 \mathrm{~min}$. (b) profile cut fitted with a quadratic polynomial yielding a radius of curvature of $950 \mu \mathrm{m}$. The depth of the structure is measured to be $495 \mu \mathrm{m}$ and the inset shows the profile along its minimum. 
FIB has been investigated. Figure 4.3(a) displays a fabricated structure with design diameter of $d=80 \mu \mathrm{m}$ and design depth $h=500 \mu \mathrm{m}$. The radius of curvature is determined by AFM measurement [cf. Fig. 4.3(b)] to be $R=950 \mu \mathrm{m}$. The profile shows a wavy pattern with amplitude of a few nanometers, especially accentuated around the center of the structure. Its origin lies in the discretization of the profile definition, in this case by a $2500 \times 2500 \mathrm{Pixel}^{2}$ bitmap file for the $80 \times 80 \mu \mathrm{m}^{2}$ exposed region, which leads to interference effects with the finite beam diameter of the focused Ga ions. This surface roughness will induce scattering loss and is therefore not useful for the purposes intended. Different parameters have been tested, i.e. shallower structure with a reduced depth $(0.06 \mu \mathrm{m})$ and a different resolution for the profile definition. Nevertheless, this structures still show oscillation in the profile of roughly $1 \mathrm{~nm}$. Similar to the profile shown in Fig 4.3(b), the shallower profile also significantly deviates from the designed parabolic shape around its center. Using a de-focused ion beam might circumvent these problems. In the scope of this work investigation in this direction has not been further pursued, because for coupling to a solid state emitter we are interested in cavities with a mode volume as small as possible. They are reached which fibers having only a small imprint with small radius of curvature. We have shown above that we have fabricated such fibers with FIB milling with outstanding properties.

\subsubsection{Further techniques}

For completeness the other techniques existing to realize a fiber-based microcavity shall be summarized here.

\section{Gluing micro-mirrors to fibers}

The technique first employed by Steinmetz et al. [226] is based on lift-off coatings, with which a microlens array is coated. A fiber is centered on top of a microlens and attached by the use of ultraviolet (UV) curing glue to the coating. Finally by pulling back the fiber, the coating is detached from the lens array. A cavity with such fibers has been employed by Muller et al. to couple to a quantum dot [187]. As these lift-off coatings cannot provide state-of-the-art minimal losses, the achievable finesse is limited. Additionally the surface roughness cannot compete with the achieved values by the before mentioned techniques $\left(\mathrm{CO}_{2}\right.$-laser, FIB) and are in fact the main limit for the finesse due to scattering loss. In the early stage of the experiments we also have successfully fabricated fiber mirrors by this approach. However, due to the limitations of these mirrors, this approach has not been further pursued.

\section{Impression with a hot metallic sphere}

Kunert et al. [227] used a special fiber (bismuth oxide), which compared to silica has a lower melting point, and a tungsten carbide sphere. Both were heated and then pressed against each other to produce an impression of the sphere on the fiber facet. Compared 
to the other methods the surface roughness $\left(\sigma_{\mathrm{RMS}} \approx 9 \mathrm{~nm}\right)$ is large. Furthermore, the feature covers the whole facet which limits the minimal mode volume (see below), which is not desirable.

\section{Etching the substrate (plane fiber - curved substrate configuration)}

Instead of fabricating the concave structure inside the fiber, the first realization of fiber based microcavity by Trupke et al. [228] made use of a substrate, into which they etched curved features. However, with a surface roughness of $2 \mathrm{~nm}$, limitation to the plane fiber-concave substrate configuration and less control of the resulting structure compared to the other methods, etching is not very suitable.

\subsubsection{Choice of parameters}

The figure of merit to characterize the emitter-cavity coupling is the cooperativity, which for an ideal emitter $C \propto \frac{g^{2}}{\kappa \gamma}$. The cavity decay rate $\boldsymbol{\kappa}$ can be related to the cavity quality factor, which depends on cavity length and finesse $\kappa \propto 1 / Q \propto 1 /(l \mathcal{F})$ and the coherent coupling rate $g$ depends on the mode volume $g^{2} \propto V_{\bmod }^{-1} \propto\left(w_{0}^{2} l\right)^{-1}$. Therefore for the cooperativity we find

$$
C_{\text {ideal }} \propto \frac{\mathcal{F}}{w_{0}^{2}} .
$$

As shown above for a broad emitter we have rather $C \propto \frac{g^{2}}{\kappa \gamma} \times \kappa / \gamma^{\star}=\frac{g^{2}}{\gamma \gamma^{\star}}$. Although the cavity quality factor determines the cavity linewidth, it does not influence the effective cooperativity. The cooperativity only depends on the mode volume:

$$
C_{\text {broademitter }} \propto V_{\text {mod }}^{-1} \text {. }
$$

Thus, for room temperature cavity coupling of the NV center, the parameter to optimize is the mode volume, which has to be as small as possible. A concave structure puts a geometrical limit to the smallest cavity length achievable as illustrated in Fig. 4.4(a). Furthermore, the cleaved fiber facets are not perfectly flat. Deviations from the ideal plane surface are especially observed at the point where the fibers have been scored with a diamond blade to define a predetermined breaking point prior to cleaving. In practical implementation there will therefore always be a small gap between the two mirrors as illustrated in Fig. 4.4(b). Assuming a wavelength of $\lambda=640 \mathrm{~nm}$ Fig 4.4(c) and (d) show the calculated minimal mode volumes achievable as function of the radius of curvature for different gaps $\delta$ and diameter of structure $D=10 \mu \mathrm{m}$ (smallest diameter in order not to be limited by clipping loss, see below) or $D=30 \mu \mathrm{m}$ (typical diameter for fabricated imprints with the $\mathrm{CO}_{2}$ laser machining method), respectively.

A gap of $1-2 \mu \mathrm{m}$ is technically achievable. A smaller diameter of the concave imprint will result in a smaller mode volume. On the other hand, losses due to diffraction become significant if the diameter of the spherical imprint $(2 a)$ approaches the mode 
(a)

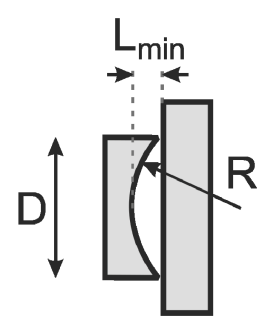

(c)

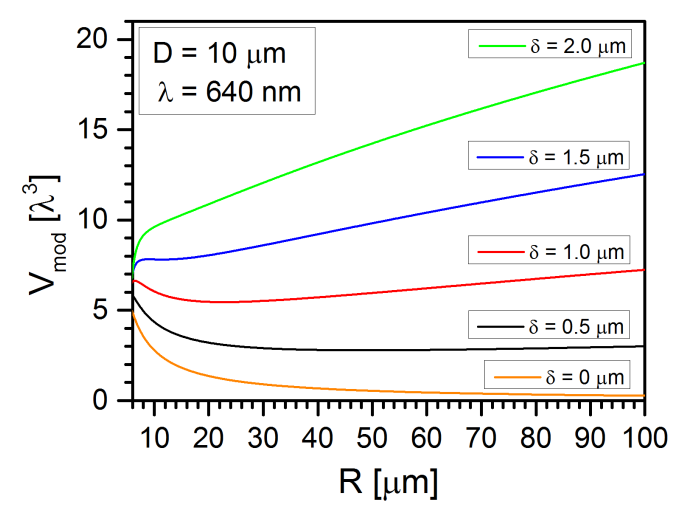

(b)

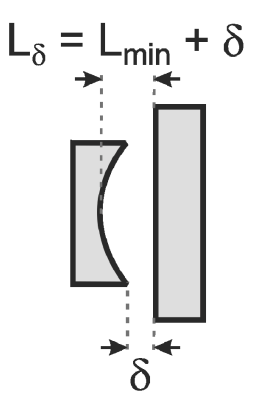

(d)

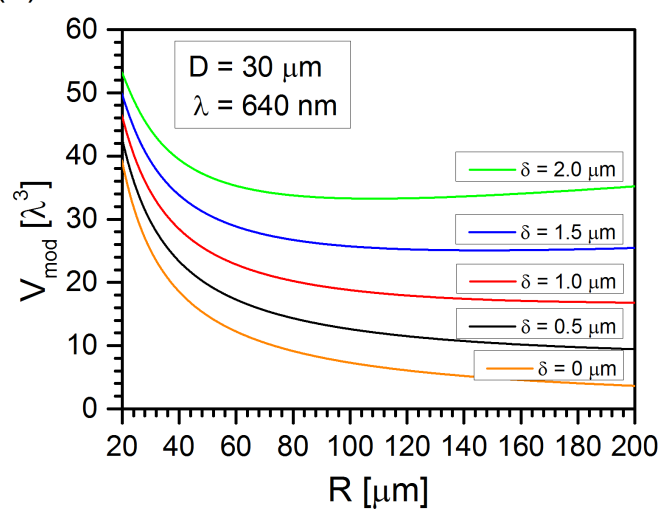

Figure 4.4: (a) The smallest achievable cavity length is limited geometrically by the concave shape (b) In practical implementations a gap $\delta$ between two mirrors will exist due to uneven fiber facets which further increase the cavity length (c) and (d) Mode volume $V_{\bmod }$ as function of radius of curvature $R$ for different gap sizes $\delta$ and diameter of the concave structure $D=10 \mu \mathrm{m}$ and $D=30 \mu \mathrm{m}$, respectively.

field diameter of the cavity mode on the mirror $(2 w)$. These clipping losses are given by $l_{\text {clip }}=\exp \left(-2 a^{2} / w^{2}\right)[222]$. Thus for a finesse greater $\mathcal{F}>10^{5}$, that can be reached with today's state-of-the-art low loss dielectric mirror coatings, the following condition must hold: $2 a>5.2 w$. For cavities with a length $l<5 \mu \mathrm{m}$ and radius of curvature $R<50 \mu \mathrm{m}$ the width of the Gaussian beam on the mirror is $w<1.9 \mu \mathrm{m}$. Thus a minimum diameter of the fabricated spherical structure of $D=10 \mu \mathrm{m}$ will be sufficient not to degrade the finesse. As mentioned above, the profile created with the $\mathrm{CO}_{2}$ laser machining technique changes from concave to convex in the outer regions. Therefore the usable diameter, the lower limit of which is given by the clipping condition, is smaller than the effective diameter of the created structure. Hence FIB milling is the method of choice on route to realize the smallest mode volumes.

One advantage of a fiber cavity is the directly fiber coupled output from the cavity. The ratio of the out-coupled light with respect to intra-cavity field not only depends on the transmission and loss coefficients of the mirror coatings, but also on the finite overlap between the cavity mode and the guided fiber mode. A mismatch between the 
two mode field diameters for fiber $\left(w_{f}\right)$ and cavity $\left(w_{m}\right.$ on the fiber mirror) and the lensing effect due to the curved fiber surface will lead to a reduced transmission by the factor $[229]$

$$
\epsilon_{\mathrm{c}-\mathrm{f}}=\frac{4}{\left(\frac{w_{f}}{w_{m}}+\frac{w_{m}}{w_{f}}\right)^{2}+\left(\frac{\pi n_{f} w_{f} w_{m}}{\lambda R}\right)^{2}},
$$

where $n_{f}$ is the refractive index of the fiber core, $\lambda$ the wavelength of the cavity and $R$ the radius of curvature on the fiber facet. Equation 4.4 assumes the fabricated structure is perfectly centered on the fiber core. In practical situations this will not be the case and will reduce the effective overlap even further. For applications requiring both large Purcell coupling and high photon output rates, e.g. high repetition rate SPS or cavity enhanced spin measurements, we use the following figure of merit (FoM) to determine the optimal design parameters: FoM $=\epsilon_{\mathrm{c}-\mathrm{f}} \times C \propto \epsilon_{\mathrm{c}-\mathrm{f}} / V_{\text {mod. }}$. Figure 4.5 (a) the FoM is plotted as function of the radius of curvature for a feature diameter of $10 \mu \mathrm{m}$. Assuming a gap in the range of $\delta=1-2 \mu \mathrm{m}$ the ideal design radius of curvature is in the range $30-40 \mu \mathrm{m}$.

When the NV-center is cooled to cryogenic temperatures, the pure dephasing of the ZPL due to phonon scattering is reduced and, as shown before (see section 2.5.4), the common Purcell regime is reached. Thus, for a cold NV center the FoM becomes $\mathrm{FoM}=\epsilon_{\mathrm{c}-\mathrm{f}} \times C \propto \epsilon_{\mathrm{c}-\mathrm{f}} / w_{0}^{2}$ assuming a constant finesse. This is displayed as function of the radius of curvature for a structure diameter $10 \mu \mathrm{m}$ in fig. $4.5(\mathrm{~b})$.

(a)

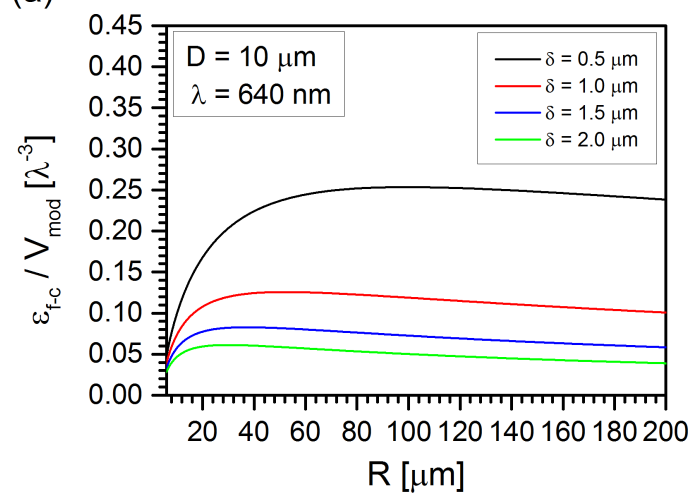

(b)

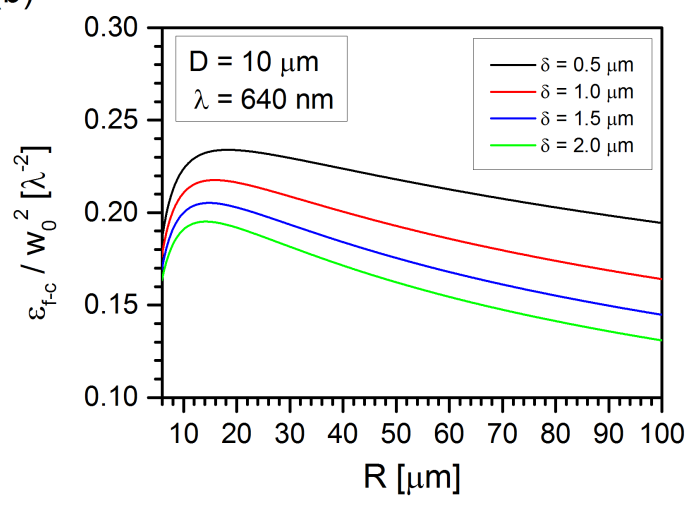

Figure 4.5: (a) Cavity out-coupling figure of merit $\epsilon_{\mathrm{c}-\mathrm{f}} / V_{\text {mod }}$ for the broad emitter case as function of the radius of curvature assuming a feature diameter of $10 \mu \mathrm{m}$. (b)(a) Cavity outcoupling figure of merit $\epsilon_{\mathrm{c}-\mathrm{f}} / w_{0}^{2}$ for the Purcell regime case as function of the radius of curvature assuming a feature diameter of $10 \mu \mathrm{m}$ 


\subsection{Technical aspects}

\subsubsection{Background emission from the fiber}

Laser light guided through an optical fiber is spatially confined to a small cross-section over the whole fiber length. The high intensity will excite fluorescence from the fiber due to dopants and impurities in the glass material. As we are interested in detecting light from a single emitter, we are very sensitive to any background fluorescence. Figure 4.6(a) displays the detected background count rate on a APD in the spectral window from $650 \mathrm{~nm}$ to $750 \mathrm{~nm}$ as function of the laser power coupled into the fiber for different types of fibers. The setup to measure the fiber fluorescence is shown in Fig. 4.7. Figure 4.6(b) contains the corresponding normalized spectra. Table 4.2 lists the tested fibers and the power dependence of the fluorescence determined by a linear fit.

Optical fibers are based on total internal reflection. To create a difference in refractive index between core and cladding either one of them is doped with impurity atoms. The fibers showing low fluorescence (S460HP and S630HP) are pure silica core fibers, whereas the other ones have a doped core, that leads to higher background fluorescence. The reduced fluorescence from the fiber core of a pure silica core fiber is also visible, when recording the fluorescence from the fiber facet with a scanning confocal micro-

(a)

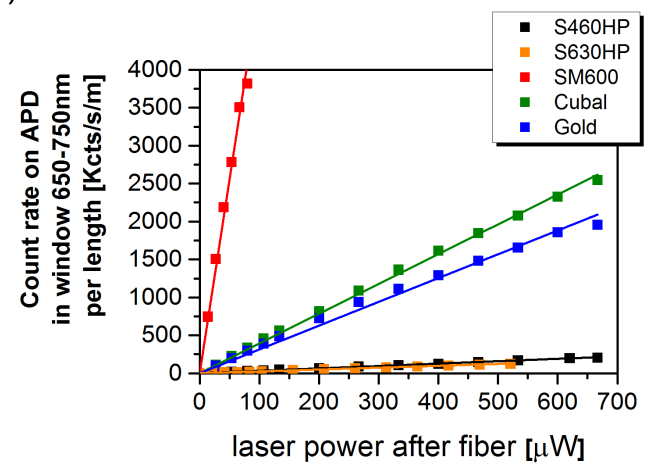

(b)

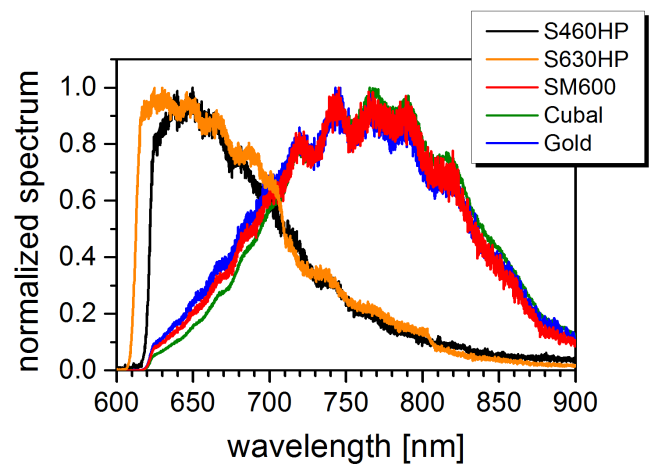

Figure 4.6: (a) Squares: measured fluorescence from different fibers (see table 4.2) in the spectral window from $650 \mathrm{~nm}$ to $750 \mathrm{~nm}$ as function of laser power at $532 \mathrm{~nm}$ coupled into the fiber. The laser power is measured after the out-coupling lens L3 (see Fig. 4.7 for a sketch of the setup) and corrected for its transmission. The count rate has been normalized to the length of the fibers, which has been on the order of $1 \mathrm{~m}$ for all the test fibers. The in-coupling efficiency, defined as ratio between power after out-coupling lens L3 and power before Brightline filter, for the different fibers were: SM600: 37\%, Cubal: 38\%, Gold: 48\%, S460HP: 24\% Lines: Linear fits to measurement points. The obtained fit parameters are listed in table 4.2. (b) Corresponding normalized spectra of background fluorescence. 


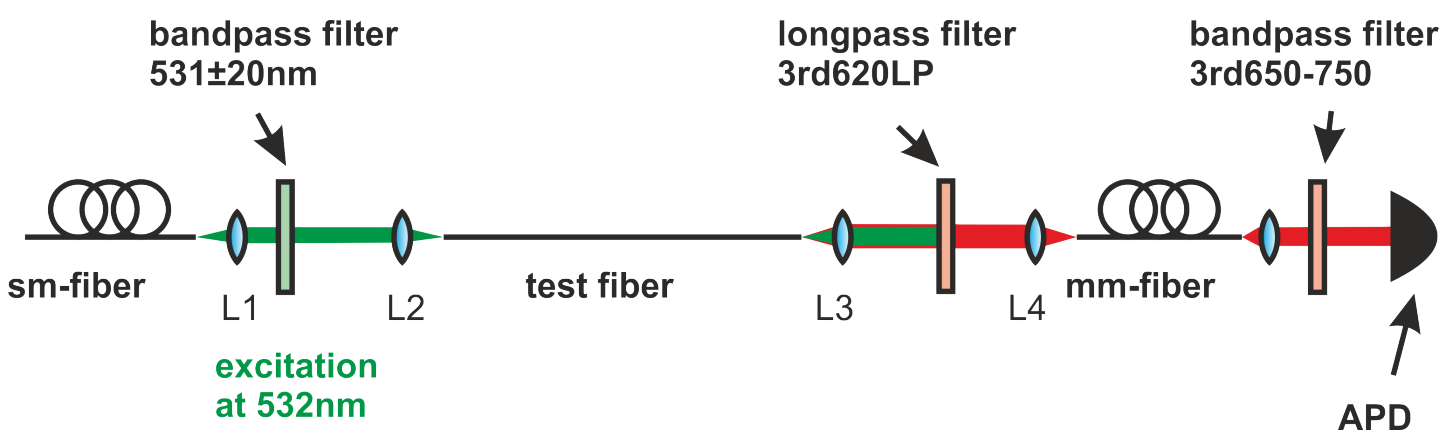

Figure 4.7: Test setup to measure fiber fluorescence: Excitation is delivered through a SM fiber. A Brightline filter (Semrock HC531/40, F39-531, transmission $T=89.9 \%$ at $532 \mathrm{~nm}$ ) ensures that only laser light at a wavelength of $532 \mathrm{~nm}$ is coupled into the test fiber. After the fiber, the excitation laser is filter out by a bandpass filter with edge at $620 \mathrm{~nm}$ (Omega optical 3rd Millenium 620LP). The fluorescence is then coupled into a MM fiber and measured by an APD (Picoquant, $\tau$-spad). In front of the APD there is an additional bandpass filter for the wavelength range from $650 \mathrm{~nm}$ to $750 \mathrm{~nm}$ (Omega Optical 3rd Millenium). The following lenses are used: L1: aspheric lens, focal length $8 \mathrm{~mm}$ (C240TME-B, Thorlabs), L2: aspheric lens, focal length $11 \mathrm{~mm}, T=96 \%$ at $532 \mathrm{~nm}$ (C220TME-A, Thorlabs), L3: 10x microscope objective, NA $0.17, T=75 \%$ at $532 \mathrm{~nm}$ (Elliot scientific), L4: aspheric lens, focal length $4.5 \mathrm{~mm}$ (C230TME-B , Thorlabs). For measurement of the S630HP fiber the two lenses L2 and L3 have been switched.

\begin{tabular}{|l|l|c|c|c|c|}
\hline \hline fiber & manufacturer & type & $\begin{array}{c}\text { MFD } \\
{[\mu \mathrm{m}]}\end{array}$ & $\begin{array}{c}\text { design } \lambda \\
{[\mathrm{nm}]}\end{array}$ & $\begin{array}{c}\text { fluorescence rate } \\
{[\text { KCts } /(\mathrm{s} \times \mu \mathrm{W} \times \mathrm{m})]}\end{array}$ \\
\hline SM600 & Fibercore & SM & 4.3 & 633,680 & 51 \\
Cubal & Oxford electronics & MM & 50 & - & 3.9 \\
Gold & Fiberguide industries & SM & 4.3 & $630-680$ & 3.1 \\
S460HP & Nufern & SM & 3.5 & $450-600$ & 0.32 \\
S630HP & Nufern & SM & 4.3 & $600-770$ & 0.24 \\
\hline \hline
\end{tabular}

Table 4.2: Fiber tested for background fluorescence in the spectral region from $650 \mathrm{~nm}$ to $750 \mathrm{~nm}$. The table lists manufacturer, main properties of the fiber such as fiber type [single mode (SM) or multimode (MM)], mode field diameter (MFD) and design wavelength. For the MM fiber the core diameter is listed instead of the MFD. The last column contains the power dependent fluorescence rate determine from a linear fit of the measurements shown in Fig. 4.6(a), which have been normalized to the fiber length. 
scope as shown in Fig. 4.8. Whereas for a doped core the doping needs to lead to an increase of the refractive index, doping of the cladding needs to result in a reduction in refractive index. Therefore, different impurity atoms are employed and the fiber type (core doped or cladding doped) can be identified by the spectrum of the fluorescence from the fiber [cf. Fig. 4.7(b)]. It is worth mentioning, that for the pure silica core fibers the measured fluorescence dramatically increases by one order of magnitude in the case, where the longpass filter, which rejects the excitation laser, is mounted exactly perpendicular in the optical beam, such that the reflected light is refocused through lens L4 onto the end-facet of the test fiber. This is because the light is not refocused on the fiber core, but rather onto the cladding and hence leads to a significant rate of fluorescence.

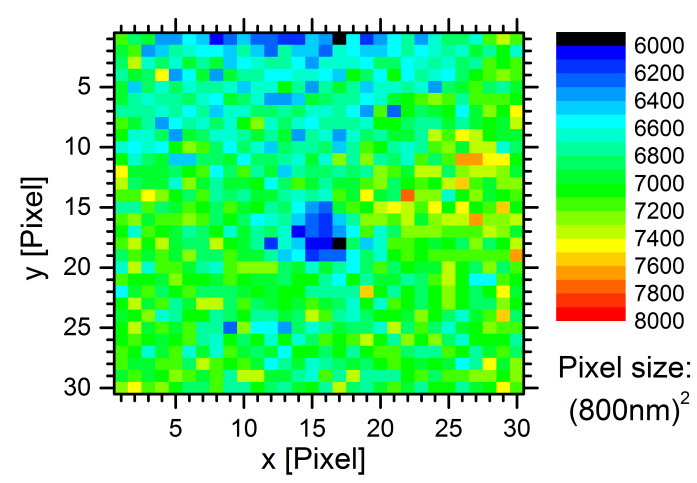

Figure 4.8: Scanning confocal microscope image with $0.7 \mathrm{~mW}$ excitation at $532 \mathrm{~nm}$ through a NA=0.55 microscope objective of the fiber facet of a pure silica core fiber (Nufern S630HP). The reduced fluorescence from the core is clearly visible.

Beside background fluorescence due to dopants, fused silica also has a typical Raman signal [230-233]. The Raman effect occurs when laser light is scattered on a molecule or solid. Interaction with local vibrations (excitation or absorption of phonons) leads to a typical shift in the laser frequency, called the Raman shift, which can be attributed to oscillation of a certain chemical bond. Figure 4.9(a) shows the spectra from an optical SM fiber (SM600), when laser light at different wavelengths [590.4 nm (from a fs-optical-parametric-oscillator-system [234]), $632.8 \mathrm{~nm}, 670 \mathrm{~nm}$ ] is coupled into the fiber. The spectra have been normalized to the laser power within the fiber. The Raman effect manifests itself by the same features occurring at the same frequency shifts and thus at different absolute wavelengths. This is illustrated in Fig. 4.9(b), which shows the spectra as function of the shift, which is characterized by the relative change in wave number $\Delta k=1 / \lambda_{\text {laser }}-1 / \lambda$. Due to different edge filters, which suppress the laser light, the lower cut-off of the spectra varies for the different excitation wavelengths. The features at $485 \mathrm{~cm}^{-1}, 600 \mathrm{~cm}^{-1}, 800 \mathrm{~cm}^{-1}, 1060 \mathrm{~cm}^{-1}$ and $1180 \mathrm{~cm}^{-1}$ are typical for $\mathrm{SiO}_{2}$. Also the maximum at around $1600 \mathrm{~cm}^{-1}$ has been reported [230]. The additional band at $\approx 685 \mathrm{~cm}^{-1}$ is due to the dopant $\left(\mathrm{GeO}_{2}\right)$ of the fiber core [230]. 
(a)

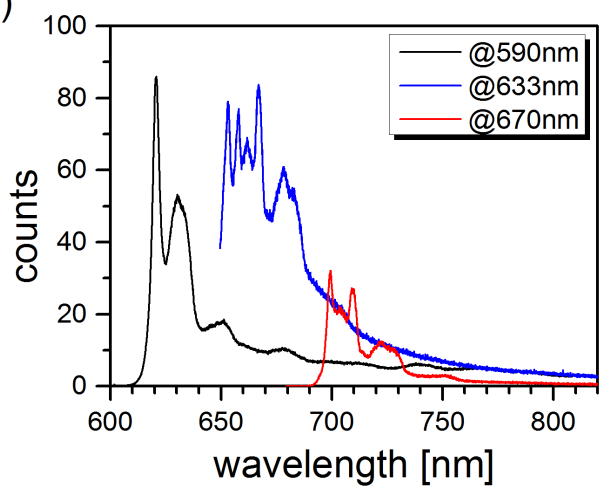

(b)

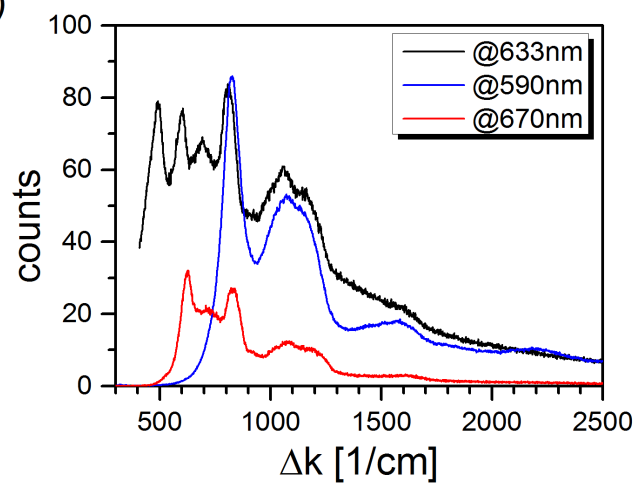

Figure 4.9: (a) Measured spectra from a single mode fiber (SM600), when laser light with wavelengths $590.4 \mathrm{~nm}, 632.8 \mathrm{~nm}$ or $670 \mathrm{~nm}$ is coupled into the fiber. The spectra have been normalized to the laser power within the fiber. For the different spectra, a long-pass filters have been used with edges at $620 \mathrm{~nm}, 650 \mathrm{~nm}$ or $700 \mathrm{~nm}$, respectively. (b) Spectra from (a) plotted as function of wave number shift $\Delta k=1 / \lambda_{\text {laser }}-1 / \lambda$.

\subsubsection{Mirror-coatings}

The design specifications for the mirror coatings on the fiber facet not only have to take into account the requirements for the cavity-coupling, but also the limitations due to the off-resonant excitation laser coupled into the fibers, as discussed in the last section. We will be using two configurations, first, a cavity with one standard plane mirror and one curved fiber-mirror and second, a cavity consisting of two fiber mirrors. The NV center will be incorporated into the cavity by depositing a ND containing a single NV centers either onto the plane mirror or one of the fibers, respectively. The NV center will be excited through the plane mirror/fiber with the ND. As output channel we will use the (second) fiber. The cavity shall be optimized for the ZPL wavelength of the NV center. The following design criteria shall be fulfilled:

1. The reflectivities of the two mirror coatings must yield the desired finesse at the ZPL wavelength.

2. The dominant output channel is the transmission of the (second) fiber.

3. The mirror, onto which the NDs are deposited, has a high transmission for the excitation laser at $532 \mathrm{~nm}$.

4. The (second) fiber has a high reflectivity for the excitation laser to suppress background fluorescence by preventing green pump light to enter.

5. The field amplitude at the position of the ND has to be maximal for the ZPL wavelength. 
6. The penetration depth for the ZPL wavelength has to be kept minimal for both coatings, in order to achieve a small mode volume.

We will aim for a moderate finesse of 3000-5000 so that additional scattering losses attributed to the ND in the cavity will still be manageable. This leads to the following specifications:

- Mirror 1 (with ND): at $\lambda=638 \mathrm{~nm} R=99.99 \%$, at $\lambda=532 \mathrm{~nm} T>95 \%$,

- Mirror 2: at $\lambda=638 \mathrm{~nm} R=99.9 \%(T=0.1 \%)$, at $\lambda=532 \mathrm{~nm} R=99.99 \%$,

where $R, T$ denote the intensity reflection or transmission coefficient, respectively. The two cavity setups under consideration with the corresponding mirror coatings are illustrated in Fig. 4.10(a) and (b).

(a)

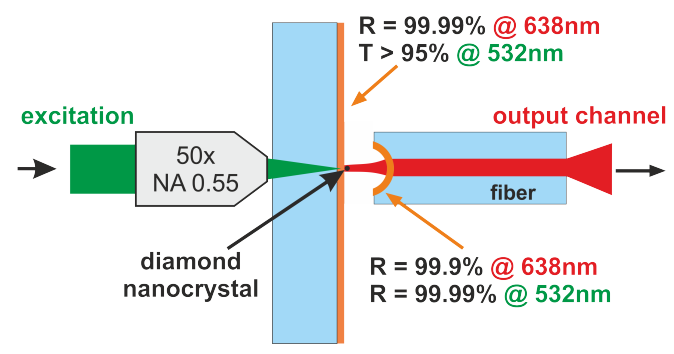

(b)

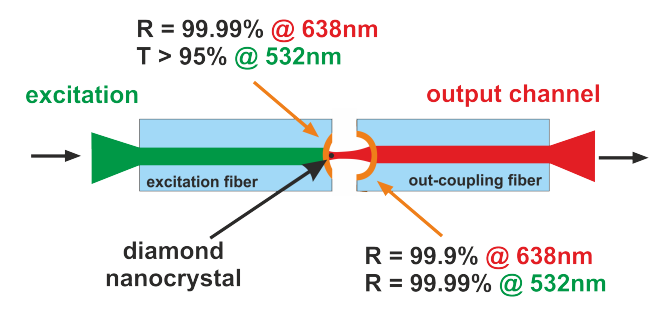

Figure 4.10: (a) Cavity consisting of a plane mirror (diameter 0.5 inch) and one fiber mirror. The excitation laser at $532 \mathrm{~nm}$ is focused with a microscope objective through the plane mirror. Light is coupled out dominantly through the fiber. (b) Fiber-fiber cavity, where both mirrors are realized on a fiber facet. Excitation of the NV center is through the first fiber, collection of the NV emission through the second.

Such high reflectivities are achieved with dielectric coatings consisting of a stack with two alternating dielectric materials with different refractive indices $\left(n_{H}>n_{L}\right)$, each with an optical thickness of a quarter of the design wavelength. Constructive interference (which is ensured by the $\lambda / 4$ thickness of the layers) of reflection at each interface will yield the high reflectivity. A typical mirror stack will be of the following order $S(H L)^{n} H$, where $S$ denotes the substrate, $H, L$ the two dielectric materials according to their relative refractive index. The coating usually ends with a layer of the high index material. For coatings in the visible spectral range typically $\mathrm{SiO}_{2}$ $\left(n_{L}=1.455\right)$ as low and $\mathrm{Ta}_{2} \mathrm{O}_{5}\left(n_{H}=2.041\right)$ as high refractive material are used.

To ensure a high field amplitude at the mirror surface as it is demanded for mirror 1 , the mirror structure needs to end with the low index material. This requires additional pairs of $\lambda / 4$-stacks to achieve the same reflectivity compared to a mirror with the high index material as last layer. The high transmission at $532 \mathrm{~nm}$ can be achieved by a slight variation in the thickness of each layer, such that at $638 \mathrm{~nm}$ the high reflectivity 
still remains, but for $532 \mathrm{~nm}$ destructive interference will lead to a maximum in the transmission.

For mirror 2, where at both wavelengths a high reflectivity is demanded, stacking of two dielectric mirrors directly on top of each other is the solution. Point 6 of the design criteria requires that first the mirror for $532 \mathrm{~nm}$ is realized on the facet, before the mirror for $638 \mathrm{~nm}$ follows. The optimization of the mirror design has to take into account the complete stack consisting of both.

Creation of such a dielectric mirror is based on thin film techniques. Three main techniques are established. Evaporative deposition, ion beam plating and ion beam sputtering (IBS). As the name indicates evaporative deposition is based on evaporation of coating material and condensation on the substrate. Ion beam plating is also based on evaporative deposition, where additionally the target is under permanent flux of ions. This yields denser molecular structure and a hard coating, whereas evaporative coating leads to a soft coating, which easily can be removed from the substrate. Mechanical cleaning (for instance wiping with a organic solvant soaked lens paper) is only possible for a hard coating. For low loss coatings IBS is the method of choice and used for the coatings in this work. A sputter target of the desired material is bombarded by ions. The sputtered atoms diffuse to the substrate where they condensate. This process also yields a hard coating.

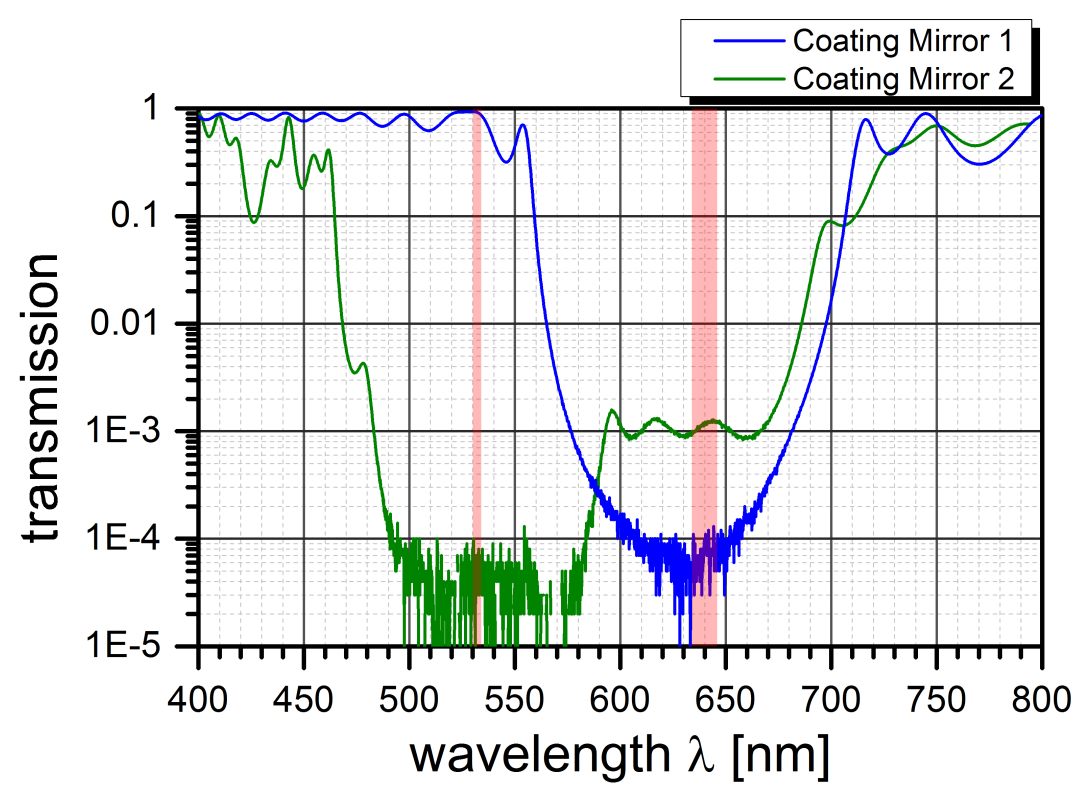

Figure 4.11: Transmission measurements of the mirror coatings employed in this work. The red highlighted spectral region is the excitation wavelength at $532 \mathrm{~nm}$ and the NV center's ZPL wavelength at $\approx 638 \mathrm{~nm}$. 
The coatings for this work have been designed and fabricated at the Laser Zentrum Hannover e.V. (LZH). Figure 4.11 shows the transmission measurements (with Hitachi U-3501) on test substrates, that have been coated simultaneously with the fibers or mirror substrates used for the cavities.

To coat the fibers, they are fixed within a thin slit of a metallic (aluminum or brass) cylinder and fixated with a piece of sheet steel. Under a microscope it is ensured that the machined fiber tips protrude the cylinder by a well defined length $(3 \pm 0.05 \mathrm{~mm})$. The cylinders are then placed, together with test substrates, into a calotte as shown in Fig. 4.12, which is mounted in the IBS chamber. The laser machined fibers from the first fabrication round (MM Cubal) have been cleaned before mounting into the calotte in ultrasonic bath, first $5 \mathrm{~min}$ in $20 \% \mathrm{HCl}$ acid, second $2 \mathrm{~min}$ in distilled water and finally $5 \mathrm{~min}$ in acetone prior to mounting in the calotte. However, cleaning of the fibers cannot eliminate all contamination, and it is therefore desirable to prevent any contamination, such that no cleaning is necessary. For later fabricated fibers, great care was taken to work under clean conditions during machining of the concave structures, such that cleaning of them afterwards was obsolete.

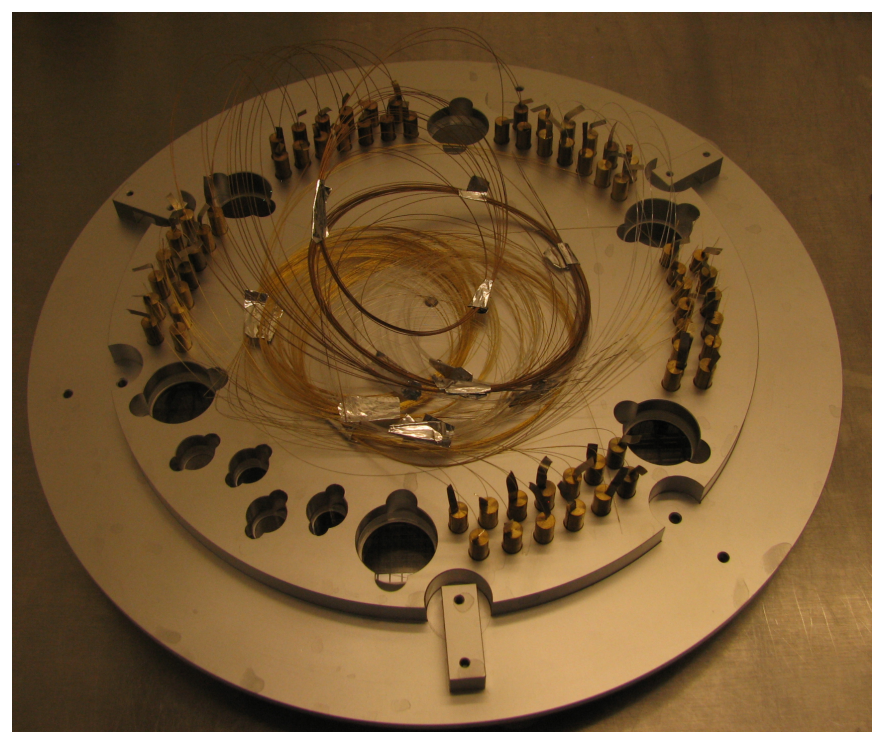

Figure 4.12: Calotte containing the fibers and test substrate used for coating by IBS

\subsubsection{Building fiber cavities}

As discussed above, two types of fiber cavities will be investigated: plane mirror-fiber and fiber-fiber cavity. In the first case the cavity consists of one fiber mirror and one plane mirror. The fiber is glued onto a home-built piezo-driven flexure stage in order to control the cavity length. The plane mirror with a diameter of 0.5 inch is mounted on a translation stage driven by piezo-motors to on one hand approach fiber and plane mirror and on the other hand to laterally scan the mirror, such that a ND on the plane 
mirror can be positioned in the cavity waist. A microscope objective, which focuses the excitation laser through the mirror onto the ND in the cavity waist, is positioned behind the plane mirror. Figure 4.13(a) shows a picture of such a cavity. The cavity is monitored from above with a stereo microscope (Zeiss Discovery.V8), in which, as exemplarily displayed in Fig. 4.13(b), the fiber and its mirror image in the plane mirror are visible. This allows to adjust angle between plane mirror and fiber and to approach the two.

The second approach consists of two fiber mirrors. Both fibers are fixed with UV curing glue onto a flexure stage after they have been aligned, such that only the cavity length remains adjustable by a piezo. A photograph of the setup, that contains two

(a)

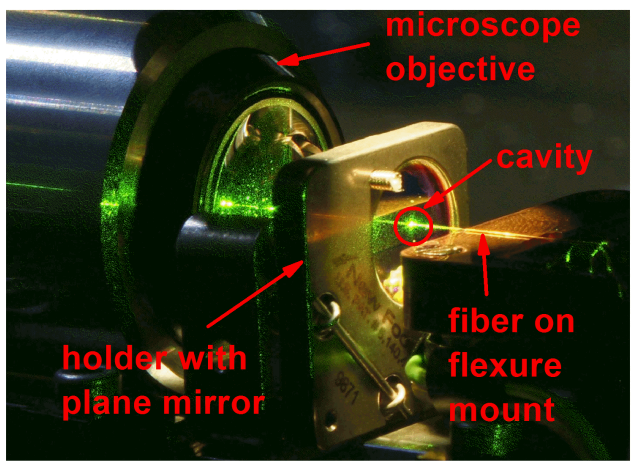

(b)

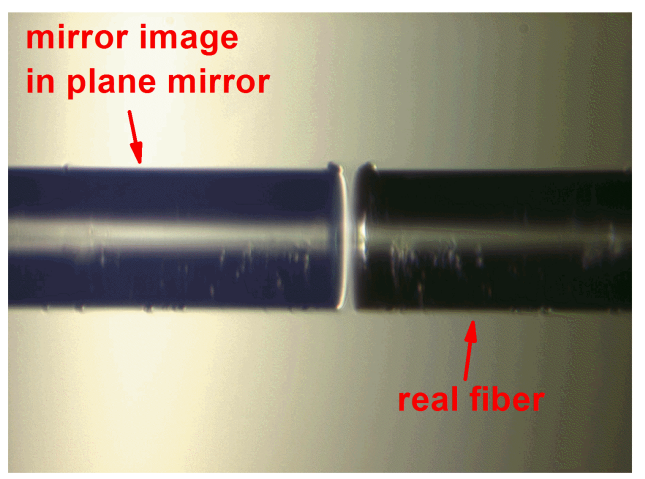

(c)

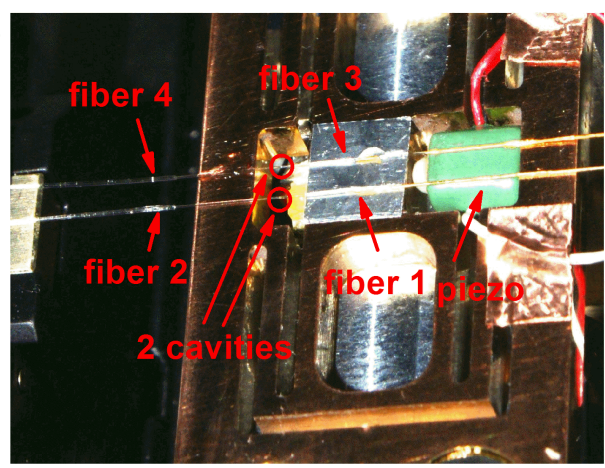

(d)

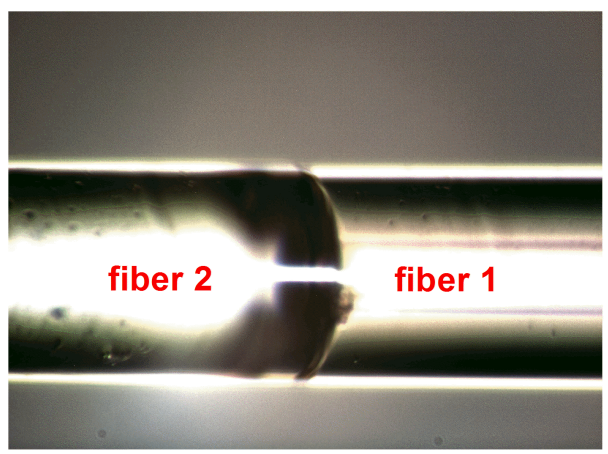

Figure 4.13: (a) Photograph of the plane mirror-fiber cavity setup. The fiber is glued onto a piezo-driven flexure mount. The plane mirror is mounted on a translation stages and can be displaced laterally and along the cavity axis by piezo motors with resolution of $80 \mathrm{~nm}$ steps. Behind the plane mirror the microscope objective to excite the ND on the plane mirror is positioned. (b) Microscope image from above of the plane mirror-fiber cavity. (c) Photograph of the setup with 2 parallel fiber-fiber cavities. Fiber 1 and 3 are glued onto the same movable part of the flexure mount driven by the piezo. Fiber 2 and 4 remain fixed in position. (d) Microscope image from above of one of the fiber-fiber cavities. 
parallel fiber cavities, is shown in Fig. 4.13(b). The purpose of using two resonators is to stabilize the cavity length as will be discussed below. A microscope image from one of the two fiber-fiber cavities is displayed in Fig. 4.13(d).

The easiest way to determine the effective cavity length is to measure the FSR $\nu_{\mathrm{FSR}}=c /(2 l)$, which is directly observable when recording the transmission spectra of a white light source through the cavity. The fundamental $\mathrm{TEM}_{00}$ modes are easily identified: compared to higher transverse modes they have the smallest resonance frequency (cf. Eq. 2.44) or equivalently they have the highest resonance wavelength. Figure 4.14 shows two such spectra for both cavity types, where the length has been reduced to the achievable minimum. The typically obtained FSR, which is on the order of $40-50 \mathrm{THz}$, corresponds to effective cavity length down to $3 \mu \mathrm{m}$. In the examples shown in Fig. 4.14 the effective cavity lengths are $3.28 \mu \mathrm{m}$ and $3.46 \mu \mathrm{m}$ for the plane mirror-fiber and fiber-fiber cavity, respectively.

(a)

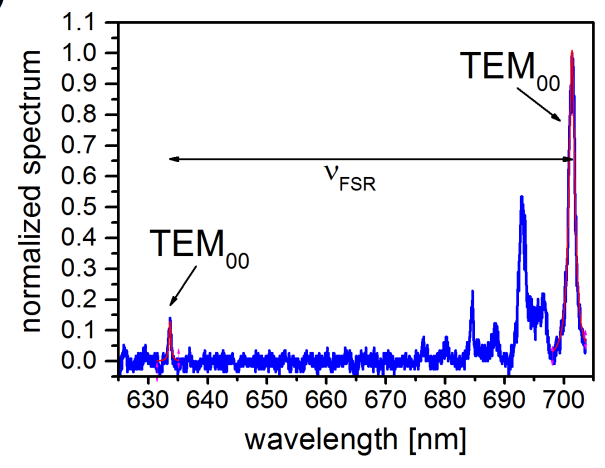

(b)

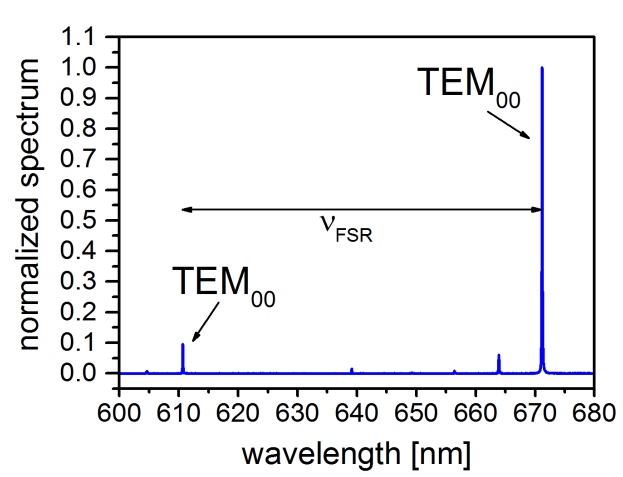

Figure 4.14: (a) Transmission spectra of a white light source through a plane mirror-fiber cavity. The FSR is $\nu_{\mathrm{FSR}}=45.6 \mathrm{THz}$ corresponding to a effective cavity length of $l=3.28 \mu \mathrm{m}$ (b) Transmission spectra of a white light source through a fiber-fiber cavity. The FSR is $\nu_{\mathrm{FSR}}=43.3 \mathrm{THz}$ corresponding to a effective cavity length of $l=3.46 \mu \mathrm{m}$

\subsubsection{Finesse}

To measure the finesse a single mode laser [external cavity diode laser (ECDL) at $640 \mathrm{~nm}$ (Toptica DL100) or widely tunable titanium:sapphire ring laser (Syrah Matisse)] is coupled into the cavity, while the cavity length is scanned. A fiber-plane mirror setup is used as shown in Fig. 4.10(a). The laser light is coupled in through the plane mirror with a microscope objective and the transmitted light through the fiber is measured on a photo diode. A polarizing beam splitter followed by a quarter wavelength plate is used to record the reflected light. (The incoming linearly polarized light transmitted by the beam splitter is turned into circular polarized light by the quarter wave plate. The reflected light, which still is circularly polarized, will by turned by the quarter wavelength plate back into linearly polarized light, but orthogonal to the incoming 
light, such that it leaves the polarizing beam splitter at the other output port, where it is detected on a photo diode.) On resonance a dip in reflection and a corresponding peak in transmission is observed, as shown exemplarily in Fig. 4.15(a). Due to non-perfect mode-matching, the incoming laser not only couples to the fundamental mode, but also higher transverse modes are excited [small peaks in transmission in Fig. 4.15(a)].

(a)

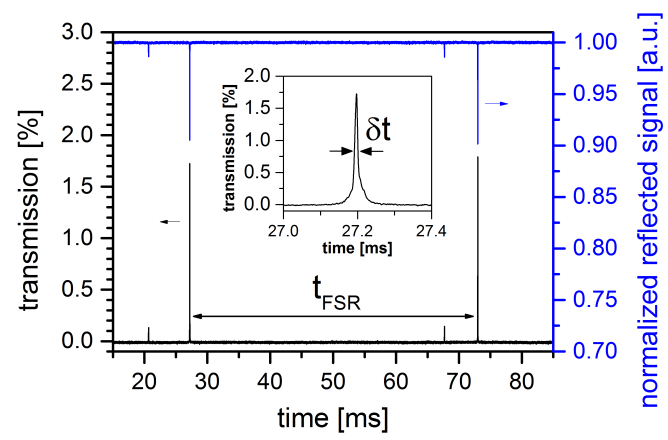

(b)

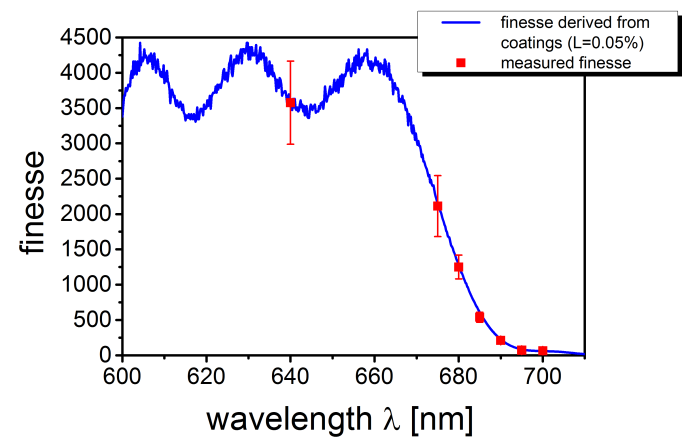

Figure 4.15: (a) Reflected and transmitted light of a single mode diode laser at $640 \mathrm{~nm}$ while scanning the cavity length. The inset shows a zoom of one of the transmission peaks. Not only is the fundamental mode observed but also higher transverse modes are visible. (b) Finesse as function of the wavelength measured at the wavelengths $640 \mathrm{~nm}, 675 \mathrm{~nm}, 680 \mathrm{~nm}$, $685 \mathrm{~nm}, 690 \mathrm{~nm}, 695 \mathrm{~nm}$ and $700 \mathrm{~nm}$. The solid line is the finesse deduced from the transmission coefficients of the the coatings (cf. Fig. 4.11) assuming an additional round-trip loss of $0.05 \%$.

The finesse is determined by the ratio of the time necessary to scan over two longitudinal modes and the width of one transmission peak:

$$
\mathcal{F}=\frac{v_{\mathrm{FSR}}}{\delta \nu}=\frac{t_{\mathrm{FSR}}}{\delta t}
$$

For this to be true, the change in cavity length needs to be with constant speed, such that time differences are proportional to changes in cavity length. Between two longitudinal modes the change in cavity length is $\lambda / 2$. Let $\delta l$ be the change in cavity length over a resonance. To derive the corresponding change in frequency we use the resonance condition

$$
\nu=n \times \frac{c}{2 l} \quad \Rightarrow \quad|\delta \nu|=n \times \frac{c \delta l}{2 l^{2}}=\frac{2 l}{\lambda} \times \frac{c \delta l}{2 l^{2}}=\frac{c}{\lambda} \frac{\delta l}{l},
$$

and hence

$$
\mathcal{F}=\frac{v_{\mathrm{FSR}}}{\delta \nu}=\frac{c / 2 l}{\frac{c}{\lambda} \frac{\delta l}{l}}=\frac{\lambda / 2}{\delta l}=\frac{t_{\mathrm{FSR}}}{\delta t} .
$$

Knowing the transmission of the mirror coatings allows deriving from the finesse the additional round-trip losses

$$
L=\frac{2 \pi}{\mathcal{F}}-T_{1}-T_{2}
$$


With a measured finesse of $\mathcal{F}=3600 \pm 600$ at $640 \mathrm{~nm}$ the losses amount to $L=$ $0.05 \pm 0.03 \%$. To a good approximation they are independent of wavelength within the range of interest, as the comparison between measurements of the finesse at different wavelengths and the finesse deduced from the known transmission and loss factors implies, as illustrated in Fig. 4.15(b). The transmission at $640 \mathrm{~nm}$ for the two mirror coatings are $T_{1}=0.007 \%$ and $T_{2}=0.117 \%$. It is worth mentioning, that for the longer wavelength, where the finesses decreases and is limited by the transmission through the mirror coatings, a slight change in the additional round-trip losses will hardly be noticeable.

The mode-matching $\epsilon$ is the ratio of the effectively coupled power into the cavity mode to the total incoming laser power. It can be determined from the measured reflected light. From Eq. 2.29 we find

$$
\epsilon=\frac{1-P_{\mathrm{refl}} / P_{\mathrm{in}}}{1-\left(\frac{-T_{1}+T_{2}+L}{T_{1}+T_{2}+L}\right)^{2}}=\frac{1-P_{\mathrm{refl}} / P_{\mathrm{in}}}{1-\left(\frac{2 \pi / \mathcal{F}-2 T_{1}}{2 \pi / \mathcal{F}}\right)^{2}}=\frac{1-P_{\mathrm{refl}} / P_{\mathrm{in}}}{1-\left(1-\mathcal{F} T_{1} / \pi\right)^{2}} .
$$

The numerator is the relative dip in reflection on resonance because off-resonantly all light will be reflected from the cavity. This is directly accessible from the measured normalized reflected signal. As the transmitted signal is measured after the fiber, the finite spatial overlap between cavity mode and guided fiber mode will reduce the measured transmission as compared to Eq. 2.28. We characterize this by the overlap fraction $\epsilon_{\mathrm{ov}}$ given by

$$
\epsilon_{\mathrm{ov}}=\frac{T_{\mathrm{res}}}{\epsilon \times T_{\mathrm{max}-\text { theo }}}=\frac{T_{\mathrm{res}}\left(T_{1}+T_{2}+L\right)^{2}}{\epsilon \times 4 T_{1} T_{2}},
$$

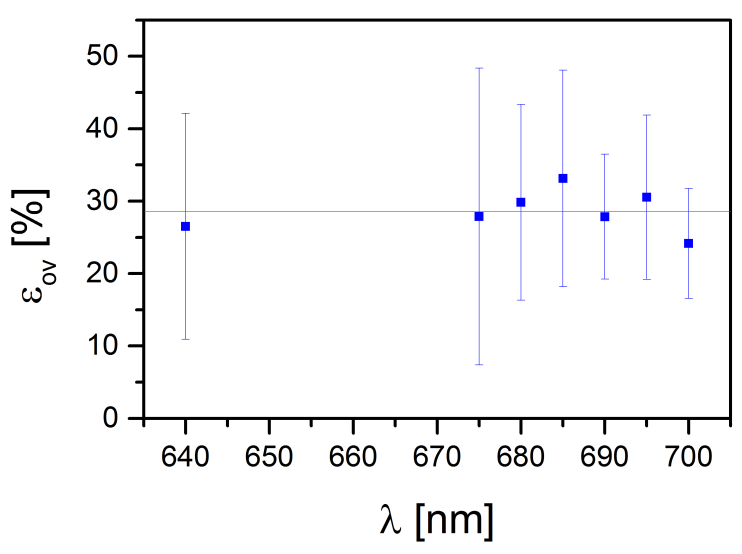

Figure 4.16: Calculated overlap between cavity mode and guided fiber mode as function of the wavelength for the measurements shown in Fig. 4.15(b). The red line shows the average, which amounts to $\epsilon_{\mathrm{ov}}=29 \pm 5 \%$. 
where $T_{\text {res }}$ is the measured transmission on resonance and $T_{\max }$-theo the theoretical maximal transmission as given by Eq. 2.28. For the case of the shown measurements in Fig. 4.15, the overlap amounts to $\epsilon_{\mathrm{ov}}=29 \pm 5 \%$ when averaging over the results obtained at the different wavelengths as shown in Fig. 4.16. Note that the limiting factor is not the difference in MFD between cavity mode and guided fiber mode or lensing effect of the concave imprint: for the given cavity parameters (cavity length $l=3.5 \mu \mathrm{m}$, radius of curvature $R=71.6 \mu \mathrm{m}$ and mode field diameter of the fiber $2 \omega_{f}=4.3 \mu \mathrm{m}$ ) this, according to Eq. 4.4, equals to $\epsilon_{\mathrm{c}-\mathrm{f}}=94 \%$. The much lower overlap is due to the displacement of the fabricated concave imprint relative to the center of the fiber core. This value is specific to the fiber used in the experiment and has to be measured separately for different fibers.

On the plane mirror there are NDs spin coated. Measurement of the finesse depends on the lateral positioning of the plane mirror. The results above show the best case. Due to scattering induced by the ND, the finesse is sometimes noticeably reduced when a ND is at the cavity waist position. Figure 4.17(a) shows exemplarily the obtained reduced finesse at a different lateral position of the plane mirror. The additional roundtrip losses at this specific position are increased to $L=0.12 \%$. However, the coupling between fiber mode and cavity mode is unaltered as the same fiber is used. Indeed the obtained values obtained for $\epsilon_{\mathrm{ov}}$ at the different test wavelengths are within the error margin with the previous results as illustrated in Fig. 4.17(b). Averaging over both measurements allows to further precise its value to $\epsilon_{\mathrm{ov}}=26 \pm 4 \%$.

(a)

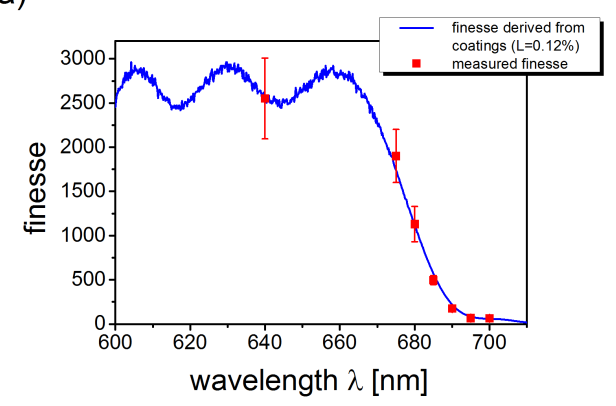

(b)

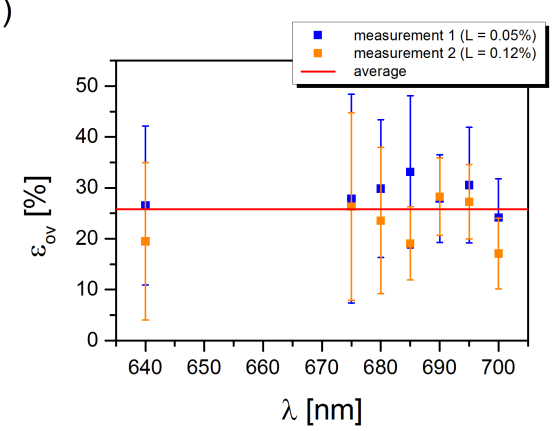

Figure 4.17: (a) Measurement of the finesse while a ND is in the cavity waist as function of the wavelength measured at the wavelengths $640 \mathrm{~nm}, 675 \mathrm{~nm}, 680 \mathrm{~nm}, 685 \mathrm{~nm}, 690 \mathrm{~nm}, 695 \mathrm{~nm}$ and $700 \mathrm{~nm}$. The solid line is the finesse deduced from the transmission coefficients of the the coatings (cf. Fig. 4.11) assuming an additional round-trip loss of $0.12 \%$. (b) Calculated overlap for the measurements shown in Fig. 4.15(b) (blue squares) and the measurements shown in (a) (orange squares). The red line shows the average over all values which equals $\epsilon_{\mathrm{ov}}=26 \pm 4 \%$. 


\subsubsection{Length stabilization schemes}

Fabry-Perot cavities have the advantage of being easily tunable by variation of their length. However, to remain at a certain resonance frequency, it is necessary to stabilize the cavity length. From Eq. 4.7 we see, in order for the resonance frequency to remain within the cavity linewidth, the maximal change in cavity length acceptable is $\delta l=$ $\lambda /(2 \mathcal{F})$. For a finesse of $\mathcal{F}=3600$ at a resonance wavelength of $640 \mathrm{~nm}$ this is $\delta l=$ $0.9 \AA \AA$.

To create an error signal, which can be used as feed-back for the cavity length control, a reference laser is coupled into the cavity and its reflected intensity is monitored. The typical laser power used for this purpose is on the order of $100 \mu \mathrm{W}$. This is 9 order of magnitude larger than the emission power from a single NV center with a count rate of $10^{6}$ photons/s, which corresponds to a power of $0.3 \mathrm{fW}$ at a wavelength of $650 \mathrm{~nm}$. To avoid background noise obscuring the NV emission due to the much stronger stabilization laser, the strategy is to spatially separate the two fields completely and to use two different cavities, which lengths are coupled and controlled simultaneously. This for instance is achieved in the plane mirror - fiber setup by gluing a second fiber on the same piezo-driven flexure mount, that already holds the fiber for the NV-cavity as illustrated in Figure 4.18. The two-cavity-approach is also realizable with two parallel mounted fiber-fiber resonators.

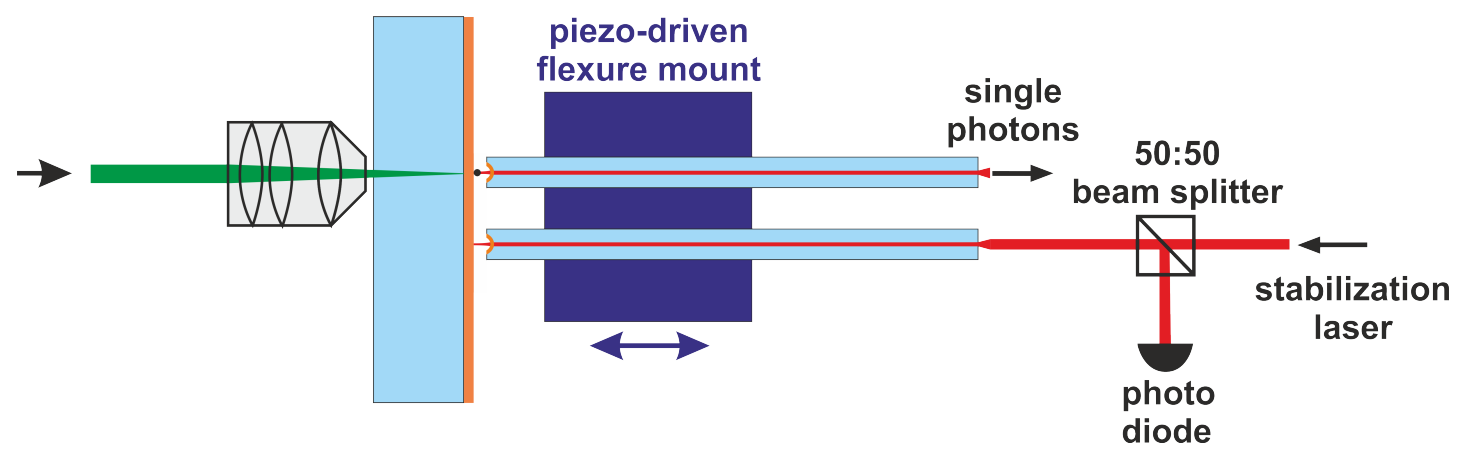

Figure 4.18: Schematic setup for the length stabilization scheme: In order to separate the low intensity field corresponding to the emission of single photons from a single NV center and the classical intensity of the stabilization laser 2 cavities are used. Their lengths are coupled and simultaneously controlled by a piezo-driven flexure mount onto which both fibers are fixed. The error signal to compensate length fluctuations is created from the measured reflection of the second cavity. As stress in an optical fiber induces birefringence a non-polarizing 50:50 beam splitter is used to be independent of polarization.

When monitoring the reflected signal from the fiber while scanning the cavity length, the dip in reflection is asymmetric as shown in Figure 4.19(a). Here the reflected and transmitted signal of a reference laser at $640 \mathrm{~nm}$ coupled into the cavity from the fiber side are displayed. (For first characterization only 1 fiber is mounted and the microscope 
(a)

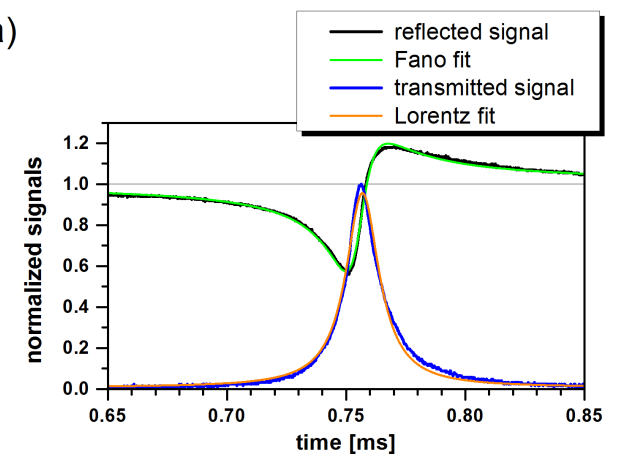

(b)

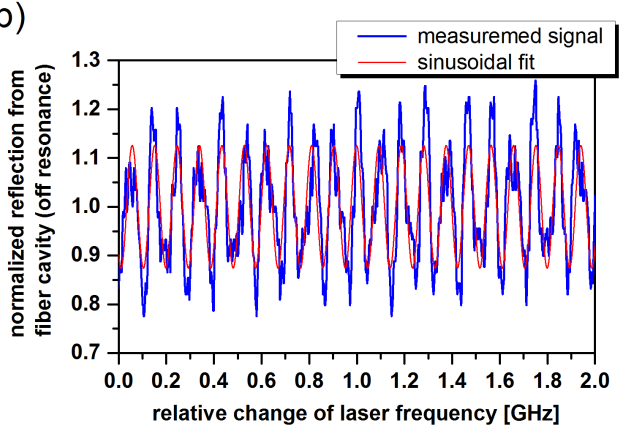

(c)

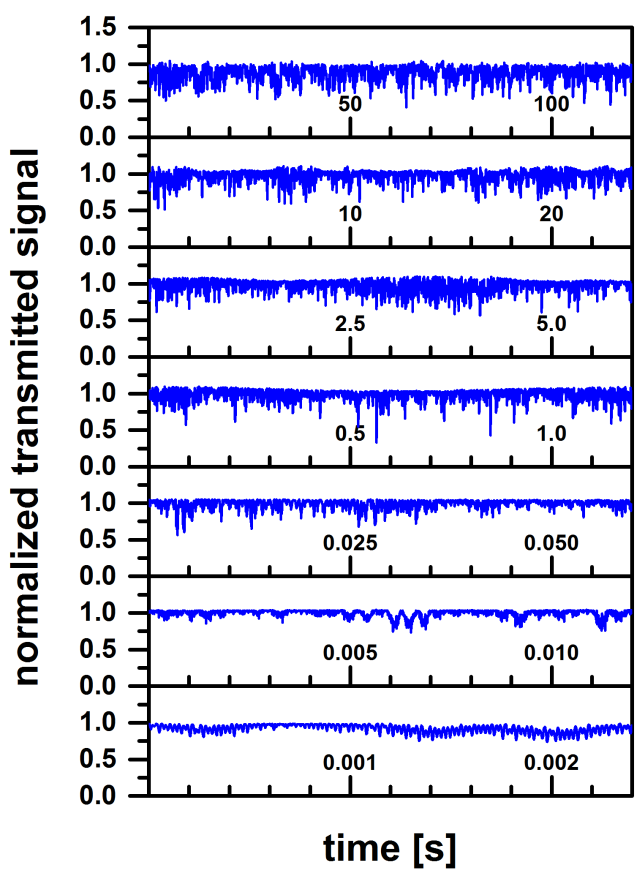

Figure 4.19: (a) Normalized reflected and transmitted signal measured on photo-diodes while monotonously scanning the cavity length. The reflected signal is fitted with a Fano profile (Eq. 4.11) and the transmitted signal with a Lorentz profile. (b) Reflected signal from the cavity, that is far off-resonant, while the frequency of the laser is swept over $2 \mathrm{GHz}$. The interference fringes are due to the fiber itself being a resonator with one high-reflective and one low-reflective end. The free spectral range of $\nu_{\mathrm{FSR}}=94 \mathrm{MHz}$ agrees with the fiber length of $l_{\text {fiber }}=1.1 \mathrm{~m}$. (c) Normalized transmitted signal measured for different times durations while the cavity length is actively stabilized using the reflected light to create an error signal.

objective employed to collect the transmitted light.) Remember that in the case shown above in Fig 4.15(a), where the laser was coupled in through the plane mirror, the reflected signal was symmetric. For the signal shown in Fig. 4.19(a), the reflected signal has the form of a Fano resonance [235], which occurs when a discrete mode couples to a continuum of modes. To understand its origin here, one has to consider that the fiber itself is a low finesse resonator with a high reflectivity at the structured side $\left(R_{1}=99.9 \%\right)$ an low reflectivity at the out-coupling end $\left(R_{2}=4 \%\right)$. The optical length $(n \times l)$ of the fiber, which is approximately $1.6 \mathrm{~m}$, leads to a free spectral range of $95 \mathrm{MHz}$. These resonances can be visualized via the reflected signal of a reference laser from a cavity, that is far off-resonance: while sweeping the laser frequency monotonously a modulation of the signal is observed, as shown in Fig 4.19(b). A mode of the cavity 
with finesse of $\mathcal{F}=3600$ and cavity length of $5 \mu \mathrm{m}$, which results in a resonance width of $\delta \nu=8.3 \mathrm{GHz}$, will thus couple to many of the resonances of the second cavity formed by the fiber itself. This is the basis for observing Fano resonances. A similar phenomenon has also been observed for photonic crystal cavities [236]. The Fano shape is characterized by the function

$$
F(t)=A_{0}+F_{0} \frac{\left[q+\frac{2\left(t-t_{0}\right)}{\delta t}\right]^{2}}{1+\left[\frac{2\left(t-t_{0}\right)}{\delta t}\right]^{2}},
$$

where $q$ measures the asymmetry of the Fano shape, $t_{0}$ is the center and $\delta t$ the width of the underlying resonance and $A_{0}, F_{0}$ are offset and amplitude factor. Fitting the reflected signal gives the parameters $q=0.68, t_{0}=75.5 \mathrm{~ms}, \delta t=16.5 \mu \mathrm{s}, A_{0}=0.58$ and $F_{0}=0.42$. A Lorentz fit of the transmitted signal gives a center of $t_{0}=75.7 \mathrm{~ms}$ and width of $\delta t=17.0 \mu \mathrm{s}$, which agrees well with the values obtained from the Fano fit.

The Fano shape gives a suitable error signal to stabilize onto the maximum of the transmission by a side-of-fringe lock of the reflected signal. Using the appropriate setpoint and adapting the correct PID parameters, that are controlled by a commercial stabilization electronics solution (Laselock TEM Messtechnik), the length can be stabilized. Figure 4.19(c) shows the transmitted signal recorded for different time durations. Transmission is always maintained, but shows occasional drops at a $10 \mathrm{~ms}$ timescale. The reason are oscillations at $\approx 480 \mathrm{~Hz}$, which best can be seen in the second lowest trace in Fig. 4.19(c) (time interval between 6-9 ms), which corresponds to a mechanical resonance frequency of the translation stage the plane mirror is mounted on. The feed-back loop is not fast enough to instantaneously compensate them.

A more elaborate and powerful technique is Pound-Drever-Hall (PDH) laser frequency stabilization [237-239]. It uses a stable reference cavity to stabilize the laser frequency, but can vice-versa also be employed inversely, such that the laser stabilizes the cavity. It is based on determining the phase of the reflected beam from a cavity, that allows to tell whether the laser frequency is above or below the resonance. To get access to the phase, the laser is modulated to create sidebands at $\nu_{c} \pm \nu_{s}$, where $\nu_{c}$ is the carrier frequency of the laser. Such sidebands can be created by modulating the phase of the laser with frequency $\nu_{s}$, for instance by modulating the current of an ECDL. This leads to an instantaneous frequency which varies in time according to

$$
\nu(t)=\nu_{c}+\nu_{s} \beta \cos \left(2 \pi \nu_{s} t\right) .
$$

Interference of the sidebands from incident and reflected beam will lead to a beat pattern sensitive to the phase, which is given by

$$
\begin{aligned}
P_{\text {refl }}(\nu)= & P_{c}|F(\nu)|^{2}+P_{s}\left[\left|F\left(\nu+\nu_{s}\right)\right|^{2}+\left|F\left(\nu+\nu_{s}\right)\right|^{2}\right] \\
& +2 \sqrt{P_{c} P_{s}}\left\{\Re\left[F(\nu) F^{\star}\left(\nu+\nu_{s}\right)-F^{*}(\nu) F\left(\nu-\nu_{S}\right)\right] \cos \left(2 \pi \nu_{s} t\right)\right. \\
& \left.\Im\left[F(\nu) F^{\star}\left(\nu+\nu_{s}\right)-F^{*}(\nu) F\left(\nu-\nu_{S}\right)\right] \sin \left(2 \pi \nu_{s} t\right)\right\} \\
& +\left(2 \nu_{s} \text { terms }\right),
\end{aligned}
$$


where $P_{c}, P_{s}$ are the powers of the incident laser at the central or sideband frequency and $F(\nu)=E_{\text {refl }} / E_{\text {in }}$ is the amplitude reflection coefficient of the cavity. $\Re$ and $\Im$ denote real and imaginary part.

In practice, to create the error signal $\epsilon$ the measured reflected signal (photo-current on the photodiode measuring the reflected light) will be mixed with a separated part of the current from the local oscillator used for phase modulation. Low-pass filtering of the output from the mixer selects the part of the reflected signal oscillating at the modulation frequency $\nu_{s}$.

There are two cases to differentiate. First slow modulation, such that the cavity linewidth $\delta \nu$ is greater than the modulation frequency $\nu_{s}<\delta \nu$. In this case the cavity follows the varying frequency and is always in equilibrium. The error signal in this case is

$$
\epsilon_{\text {slow }} \approx 2 \sqrt{P_{c} P_{s}} \frac{d|F|^{2}}{d \nu} \nu_{s} .
$$

The second case is the sideband resolved regime, where on resonance only the central frequency is coupled into the cavity and the sidebands are reflected $\left(\nu_{s}>\delta \nu\right)$. In this case the error signal reads

$$
\epsilon_{\text {sideband-resolved }} \approx-2 \sqrt{P_{c} P_{s}} \Im\left[F(\nu) F^{\star}\left(\nu+\nu_{s}\right)-F^{\star}(\nu) F\left(\nu-\nu_{s}\right)\right] .
$$

The error signal for the two cases are plotted in Fig. 4.20.

For fiber cavities lengths down to $3 \mu \mathrm{m}$ have been achieved. Hence, the free spectral range is $\nu_{\mathrm{FSR}}=50 \mathrm{THz}$. With a finesse of $\mathcal{F}=3600$ this leads to a cavity linewidth of $\delta \nu=14 \mathrm{GHz}$. The sideband resolved regime is preferable, as the range of frequency changes, which the error signal can catch and correct for, is much larger as compared

(a)

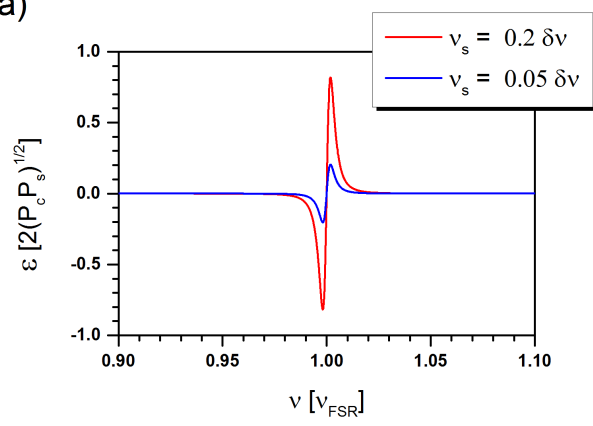

(b)

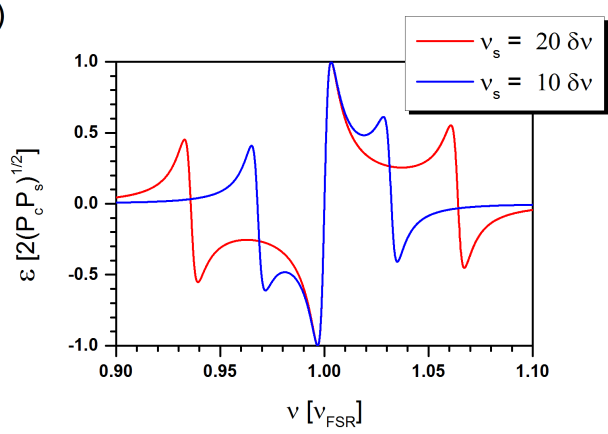

Figure 4.20: Error signal in case of slow modulation, where the modulation frequency $\nu_{s}$ is smaller as the cavity linewidth $\delta \nu$ (a) and in the opposite case of the sideband resolved regime (b). As illustrative example an amplitude damping coefficient of $r=0.99$ for the cavity was assumed corresponding to a Finesse of $\mathcal{F}=310$. The frequency is scaled in units of the free spectral range and the error signal according to the incident power of carrier $P_{c}$ and sideband $P_{s}$ wave. 
(a)

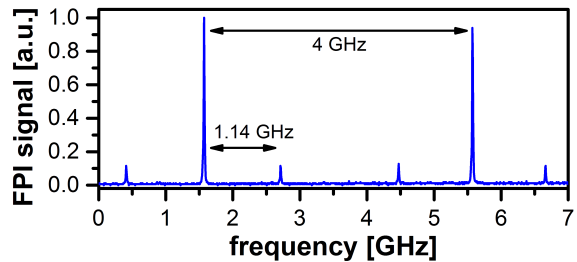

(b)

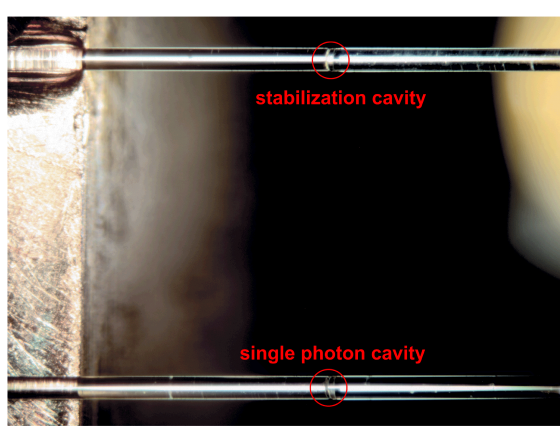

(c)

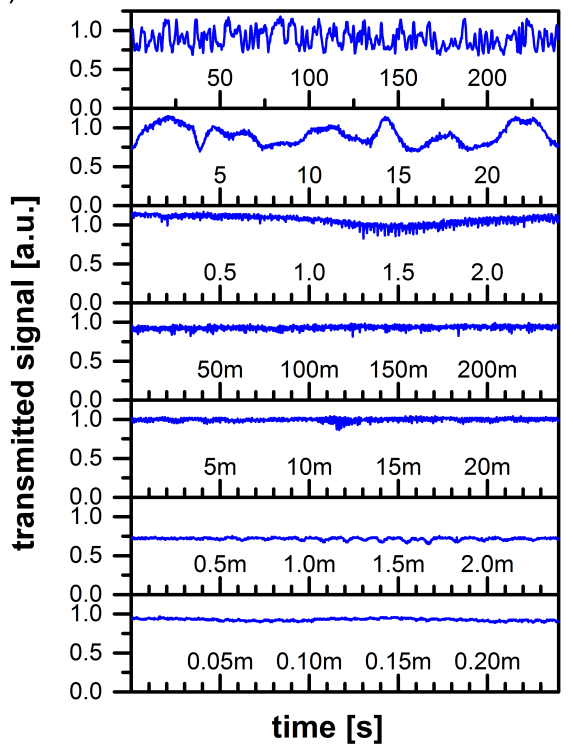

Figure 4.21: (a) Sidebands of the modulated ECDL at wavelength $\lambda=640 \mathrm{~nm}$ modulated with a frequency of $\nu_{s}=1.14 \mathrm{GHz}$ measured with a FPI with FSR $4 \mathrm{GHz}$ (Toptica FPI-1000750-4). (b) Microscope image of two parallel fiber-fiber cavities. The two left fibers remain fixed in position. The two right fibers are glued onto the same moveable element of a piezodriven flexure mount. (c) Transmission of the reference laser through the stabilization cavity recorded for different timescales, while cavity length is actively stabilized via using the reflected light to create a PDH error signal.

to the slow modulation case. However, in case of the small fiber cavities to enter the sideband resolved regime is technically demanding and only few commercial standardcomponents at the frequencies necessary are available. Thus, within this work only the slow modulation case will be employed. For this purpose the current of an ECDL at $640 \mathrm{~nm}$ is modulated with a frequency of $\nu_{\bmod }=1.14 \mathrm{GHz}$. In Fig. 4.21(a) the signal from the modulated laser coupled into a Fabry-Perot interferometer (FPI) with a FSR of $4 \mathrm{GHz}$ is shown. The created sidebands at $\nu_{s}=1.14 \mathrm{GHz}$ are clearly visible. The detailed components employed such as oscillator, amplifiers, variable attenuator, mixer, and phase-shifter are listed in the Appendix B.

In the following we discuss the case, where we adopt the PDH technique for length stabilization of a fiber-fiber cavity setup. A microscope image of the setup with two parallel cavities is shown in Fig. 4.21(b). 
(a)

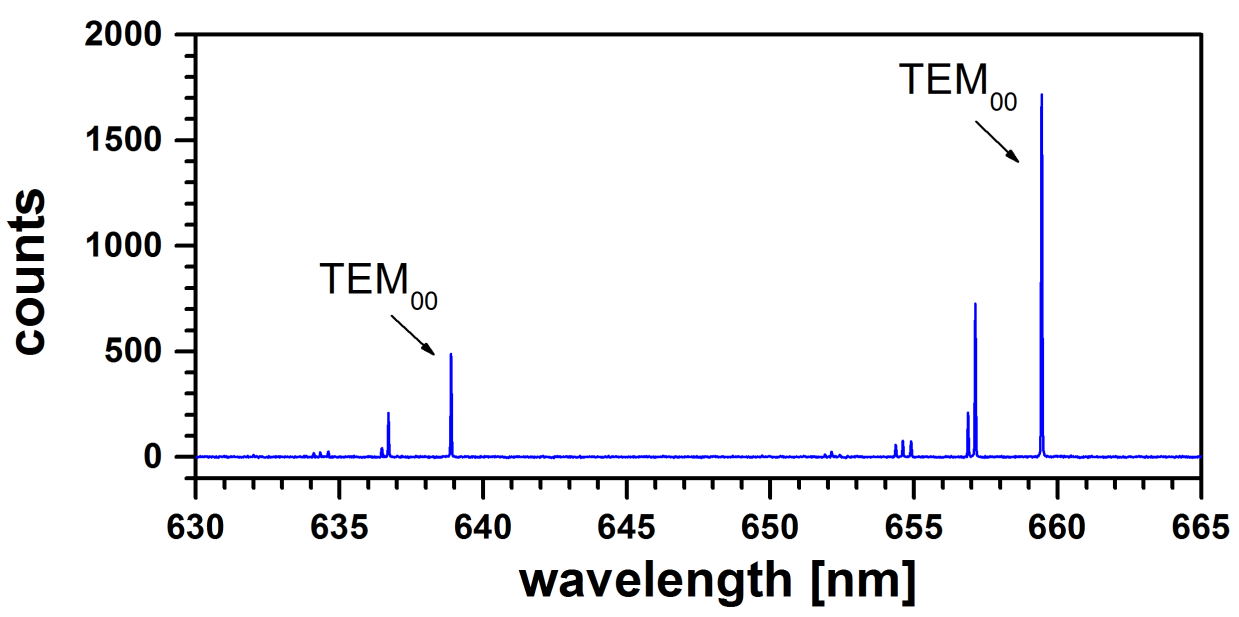

(b)

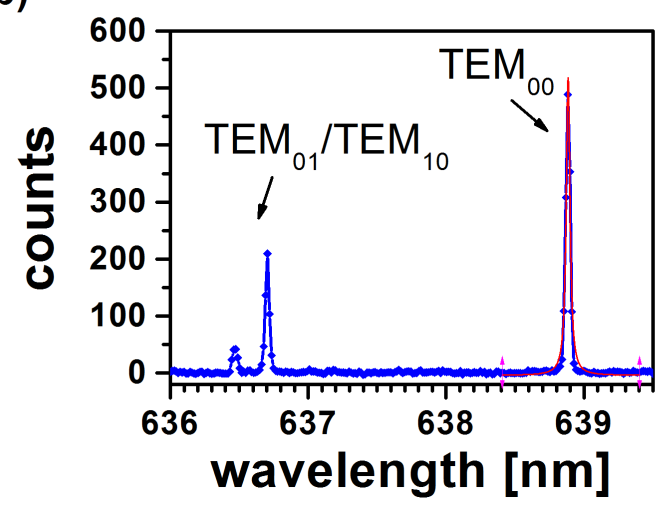

(c)

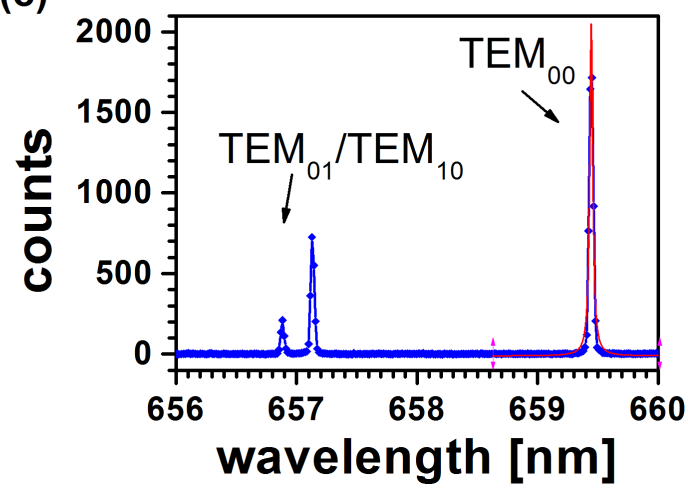

Figure 4.22: (a) White light transmission spectra of a fiber-fiber cavity. The free spectral range is $\nu_{\mathrm{vsr}}=13.63 \mathrm{THz}$, which corresponds to a cavity lenght of $l=10.24 \mu \mathrm{m}$. (b)\&(c) Zoom of the fundamental modes and first higher order transverse modes. Due to a slight ellipticity of the concave imprint in the fiber facets, the degeneracy of the $\mathrm{TEM}_{01}$ and $\mathrm{TEM}_{10}$ is lifted.

First we characterize the properties of the cavity used for stabilization. The FSR is determined by measuring the transmission spectrum of a white light source, that is plotted in Fig. 4.22. We find $\nu_{\mathrm{FSR}}=13.63 \mathrm{THz}$. This corresponds to a cavity length of $10.24 \mu \mathrm{m}$. To determine the Finesse of the cavity, the length is scanned and the cavity transmission of the reference laser at $640 \mathrm{~nm}$ (without sidebands) is recorded. This gives $\mathcal{F}=3150 \pm 170$. Hence, the cavity linewidth is $\delta \nu=4.3 \mathrm{GHz}$, which is a factor 3.6 larger than the modulation frequency of the ECDL.

To maintain the cavity in resonance with the reference laser, i.e. to maintain the maximum in transmission, a PDH error signal with suitable PID parameters is fed back to the piezo that controls the cavity length. (For this the modulation has been turned on.) The maintained transmission is shown in Fig. 4.21(c) for different time 
durations. There are some fluctuations visible on a timescale of a few seconds, which can be attributed to interference between the cavity mode and the standing wave within the fiber itself, which changes over time due to thermal fluctuations. Note that these are not fluctuations of the cavity length. This statement is verified by consecutive recording of the cavity transmission as function of the cavity length as illustrated in Fig. 4.23: the maximum transmission (maximum of the Lorentzian line shape) varies from measurement to measurement within $\pm 20 \%$.

(a)

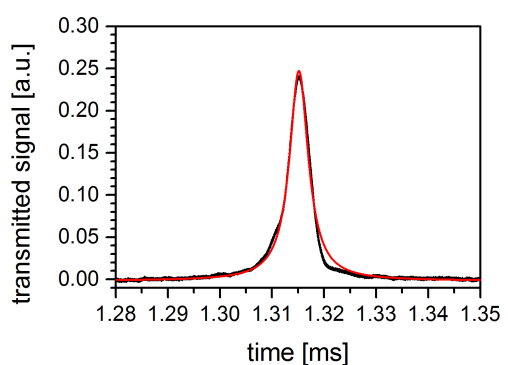

(b)

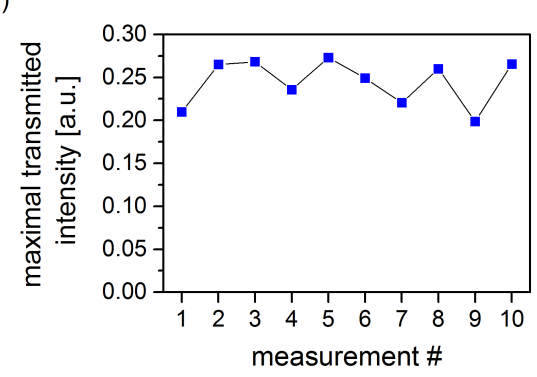

Figure 4.23: (a) Transmitted signal while scanning the cavity length over a resonance (b) Maximal intensity of the transmitted signal for 10 consecutive measurements as shown in (a).

Compared to the earlier discussed side-of-fringe stabilization technique, we here achieve with a PDH scheme a much higher length stability (compare Fig. 4.19(c) with Fig. 4.21(b)).

However, for the experiments shown in the following section, where a single NV center is coupled to a fiber cavity at room temperature, stabilization is not needed because the coupling efficiency between cavity and NV center at room temperature is not very sensitive to slight changes in the cavity resonance wavelength. For experiments at cryogenic temperature, where for an efficient coupling the cavity needs to be in resonance with the ZPL frequency, length stabilization will be needed. We have here demonstrated that this is possible and is in principle available in our lab. 


\section{Chapter 5}

\section{Single nitrogen-vacancy center coupled to a microcavity}

This chapter discusses the experimental realization of a single NV center coupled to a fiber-based microcavity at room temperature. The main focus of this chapter lies on the first part, that covers the coupling of a specific NV center, whose emission into free space has already been characterized, to a plane mirror-fiber cavity. The second part will show results from a fiber-fiber cavity approach.

\subsection{Single nitrogen-vacancy center coupled to a plane mirror- fiber cavity}

First we will identify a ND within the cavity, whose free-space emission we have already measured before (see Sects. $3.2 \&$ 3.3). Measuring the intensity correlation function of the out-coupled emission from the cavity allows to verify, that the internal dynamics is not changed as expected from theory. We will focus on two specific situations, first, on a cavity that is in resonance with the ZPL and second, a cavity which resonance wavelength corresponds to the maximum of the PSB. Detailed quantitative analysis will show a good agreement between measurement and theoretical prediction. We also will tune the cavity length over several FSRs in small steps. By this we can continuously change the coupling strength.

\subsubsection{Identification of a certain nitrogen-vacancy center}

We replace the microscope objective used for collection in Fig. 3.2(b) with a fiber to result in the cavity setup shown in Fig. 4.10(a). The parameters of the fibers in use are: the imprint on the facet has a radius of curvature $R=71.6 \mu \mathrm{m}$, a diameter $D=20.6 \mu \mathrm{m}$ and a maximal depth $z_{\max }=1.9 \mu \mathrm{m}$. 
To investigate the very same NDs as before, the excitation path is unaltered such that the focus on the plane mirror remains unchanged. The fiber with a mirror coating on its facet is mounted in front of the plane mirror and aligned, such that it is centered with respect to the excitation spot. Even though the coating on the fiber facet has a very low transmission for the excitation laser, the transmitted intensity is still detectable. It is maximized in order to align the fiber. The output from the fiber is then coupled into a MM fiber, that is connected either to APDs or a spectrometer. To filter out the residual excitation light, we use the same longpass filter as before with a transmission edge at $620 \mathrm{~nm}$.

To identify a certain ND we perform a lateral scan of the mirror and record the output from the fiber with an APD (MPD PDM PD5CTC). This scan is then overlapped

(a)

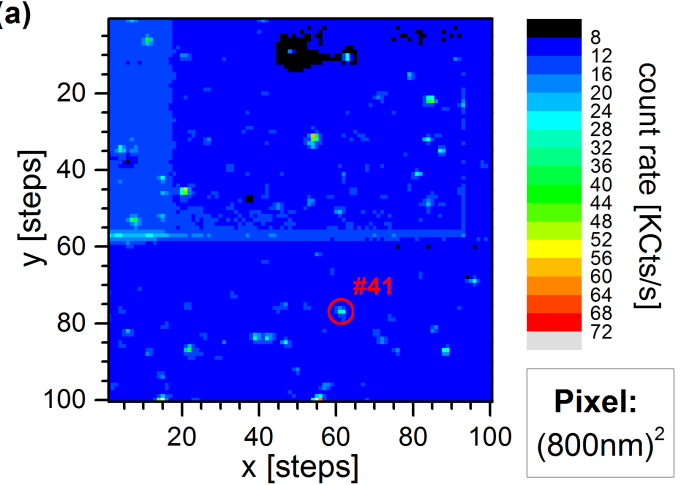

(b)

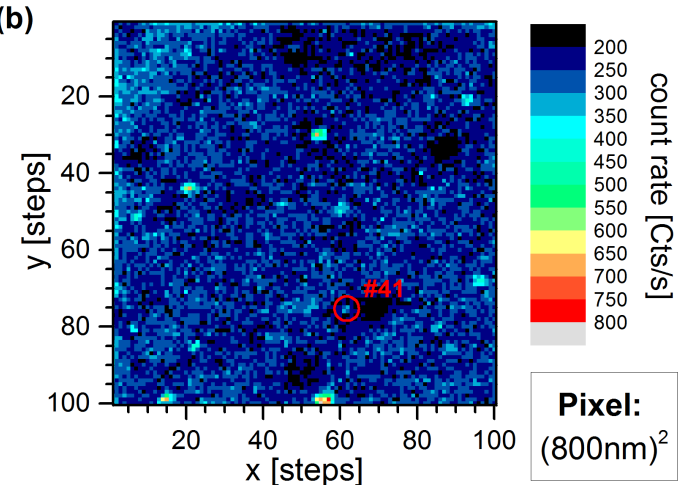

(c)

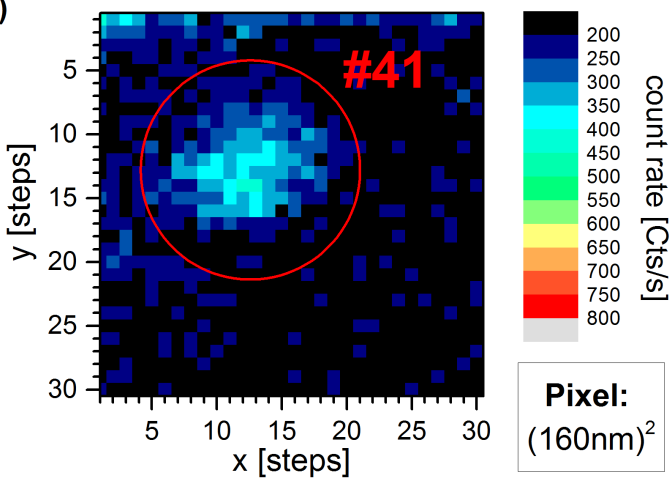

Figure 5.1: (a) Cutout from the confocal scan of the mirror in free space shown before in Fig. 3.4. The excitation laser is focused through the plane mirror onto the mirror surface and a second microscope objective in front of the mirror collects the fluorescence. (b) Scan over the same region on the mirror, where the emission into the fiber cavity is recorded. The cavity has been realized by replacing the collection objective with a fiber mirror. The ND \#41 containing a single NV center, whose cavity coupled emission will be investigated further, is marked in both scans. (c) Detailed scan with higher resolution of the cavity-coupled emission of ND \#41. 
with the scanning confocal image of the emission into free space. Figure 5.1 shows both scans, where in (a) the emission into free space is displayed (a cutout from the four scans of Fig. 3.4), and in (b) the emission from the fiber-cavity is shown. Despite the cavity-coupled scan reveals not as many details, the positions of the emitters on both scans clearly agree. We are going to further investigate the cavity coupled emission of ND \#41, that contains a single NV center. A more detailed scan with higher resolution of this ND is shown in Fig. 5.1(c). Fitting the spot with a Gaussian profile yields a localization (half-width-at- $1 / e^{2}$-of-maximum) of $w=0.9 \mu \mathrm{m}$. Note that this is smaller than the cavity waist $\omega_{0} \approx 2 \mu \mathrm{m}$. It is defined by the focus size of the excitation laser and agrees with the results obtained before (cf. Fig. 3.5). This detailed scan allows to preliminary position the ND in the cavity waist. Precise alignment will be achieved by optimizing the coupling to the fundamental $\mathrm{TEM}_{00}$ mode while observing the emission spectra as described below.

\subsubsection{Spectrum and emission rate from the cavity-coupled nitrogen- vacancy center}

Using a grating spectrometer with a CCD detector cooled by liquid nitrogen (for details see appendix B), the spectrum of the emission from the cavity-coupled NV center is recorded. Only the cavity modes on a flat baseline are observed. No signature from the broad NV spectrum of the free space emission is visible. Two such spectra are shown in Fig. 5.2 recorded at two different cavity lengths. Not only emission into the fundamental mode but also into higher transverse modes is observed. By transverse alignment of the ND with $80 \mathrm{~nm}$ wide steps, the coupling to the fundamental mode is maximized and coupling to higher transverse modes minimized. Figure 5.2 shows the optimized case.

In the first case [Fig. 5.2(a)], where the cavity has an effective length of $l=3.5 \mu \mathrm{m}$,

(a)

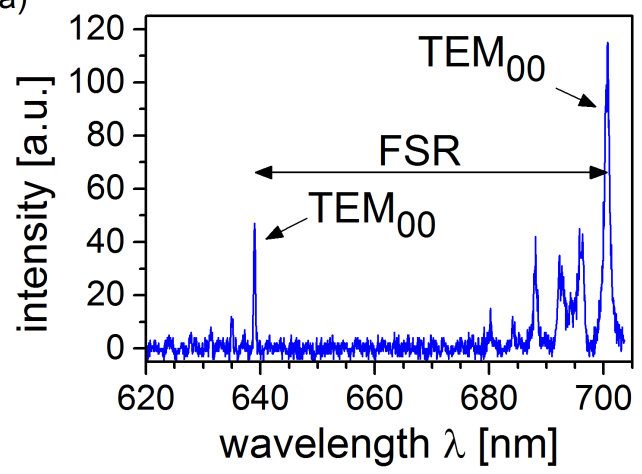

(b)

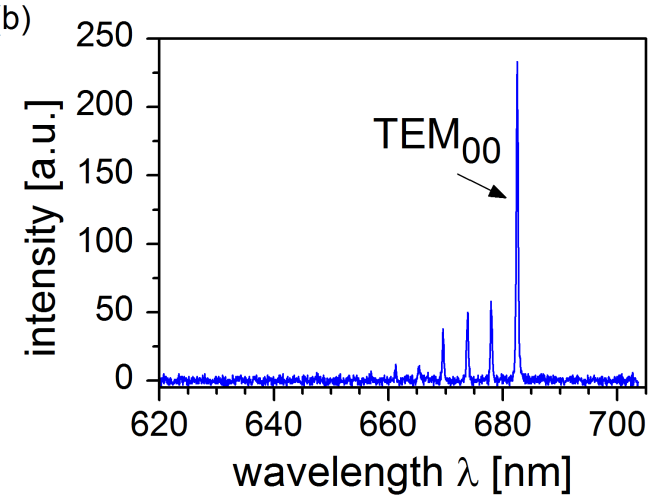

Figure 5.2: Spectrum of the cavity-coupled emission of ND \#41, where in (a) the effective length of the cavity is $l=3.5 \mu \mathrm{m}$ and in (b) $l=3.1 \mu \mathrm{m}$. 
one longitudinal mode is in resonance with the ZPL. A neighboring longitudinal mode, that has a resonance wavelength of approximately $700 \mathrm{~nm}$, is also visible in the spectrum. Hence, the FSR is directly accessible from the spectrum. From this the cavity length is deduced. The mode at $639.3 \mathrm{~nm}$ corresponds to a longitudinal mode number of $n=11$, the neighboring mode at $\approx 700 \mathrm{~nm}$ to $n=10$.

In the second case [Fig. 5.2(b)] the resonance wavelength of the mode at $682.5 \mathrm{~nm}$ is at the maximum of the PSB spectrum. The cavity length has been slightly reduced to $3.1 \mu \mathrm{m}$ in small steps, where at each length the spectrum has been recorded, and the change in resonance wavelength of each mode was monitored. This allowed to unambiguously identify the longitudinal mode number for this mode shown here as $n=9$ (see also Sect. 5.1.4).

In both cases the saturation count rate of the cavity emission is determined. The emitted photon flux as function of the power of the excitation laser is spectrally filtered and detected on APDs. Two APDs of a HBT setup (see appendix B) are used and their signals summed up. Identical bandpass filters in front of both APDs are employed. For the $3.5 \mu \mathrm{m}$ cavity with a fundamental mode at the ZPL wavelength the filters cover the range $633-647 \mathrm{~nm}$, whereas for the case of the $3.1 \mu \mathrm{m}$ cavity, where a fundamental mode is a the PSB maximum, filters with a transmission band $650-750 \mathrm{~nm}$ are used. The obtained raw data are plotted in Fig. 5.3(a) and (b) and fitted with a saturation curve including a linear background according to

$$
\mathrm{CR}=\mathrm{CR}_{\infty} \times \frac{P}{P+P_{\text {sat }}}+\sigma_{\mathrm{bg}} \times P
$$

where $\mathrm{P}$ is the excitation power measured in front of the microscope objective. We find $I_{\infty}=1040$ Counts $/ \mathrm{s}$ and $P_{\text {sat }}=0.72 \mathrm{~mW}$ for $l=3.5 \mu \mathrm{m}$ and $I_{\infty}=6200$ Counts $/ \mathrm{s}$ and $P_{\text {sat }}=0.46 \mathrm{~mW}$ for $l=3.1 \mu \mathrm{m}$. The background rate, that has also been fitted, amounts to $\sigma_{\mathrm{bg}}=83$ Counts $/(\mathrm{smW})$ and $\sigma_{\mathrm{bg}}=425$ Counts $/(\mathrm{smW})$, respectively.

To separate the intensity within the fundamental mode from the also detected photons within the higher transverse modes that lie within the transmission window of the filter in front of the APD, the spectrum is fitted as shown in Fig. 5.3(c) and (d). In the first case, where the fundamental mode is in resonance with the ZPL, $74 \%$ of the detected photons are within the fundamental mode, such that the effective count rate amounts to $\mathrm{CR}_{\infty}\left(\lambda_{\mathrm{ZPL}}\right)=770$ Counts/s. In the second case, where the resonance wavelength of the fundamental mode is at the PSB maximum, $60 \%$ of the detected photons are within this mode. Thus the effective count rate is $\mathrm{CR}_{\infty}\left(\lambda_{\mathrm{PSB}}\right)=3700$ Counts/s.

The length of the cavity is not actively stabilized. The width of the mode therefore is determined by the fluctuations in the cavity length during the time necessary to record the spectra $(30 \mathrm{~s})$ and not limited by the finesse of the cavity. The fits shown in Fig. 5.3(c) and (d) yield for the fundamental mode a FWHM of $\Delta \lambda=0.37 \mathrm{~nm}$ and $\Delta \lambda=0.39 \mathrm{~nm}$, respectively. They correspond to a frequency width of $\Delta \nu=270 \mathrm{GHz}$ and $\Delta \nu=250 \mathrm{GHz}$, whereas the cavity linewidths determined by the finesse are $\delta \nu=$ $46 \mathrm{GHz}$ and $\delta \nu=90 \mathrm{GHz}$ as calculated below (see table 5.4). Using $\Delta l=n / 2 \times \Delta \lambda$ the fluctuations in the cavity lengths compute to $\Delta l=2.0 \mathrm{~nm}$ and $\Delta l=1.8 \mathrm{~nm}$, respectively. 

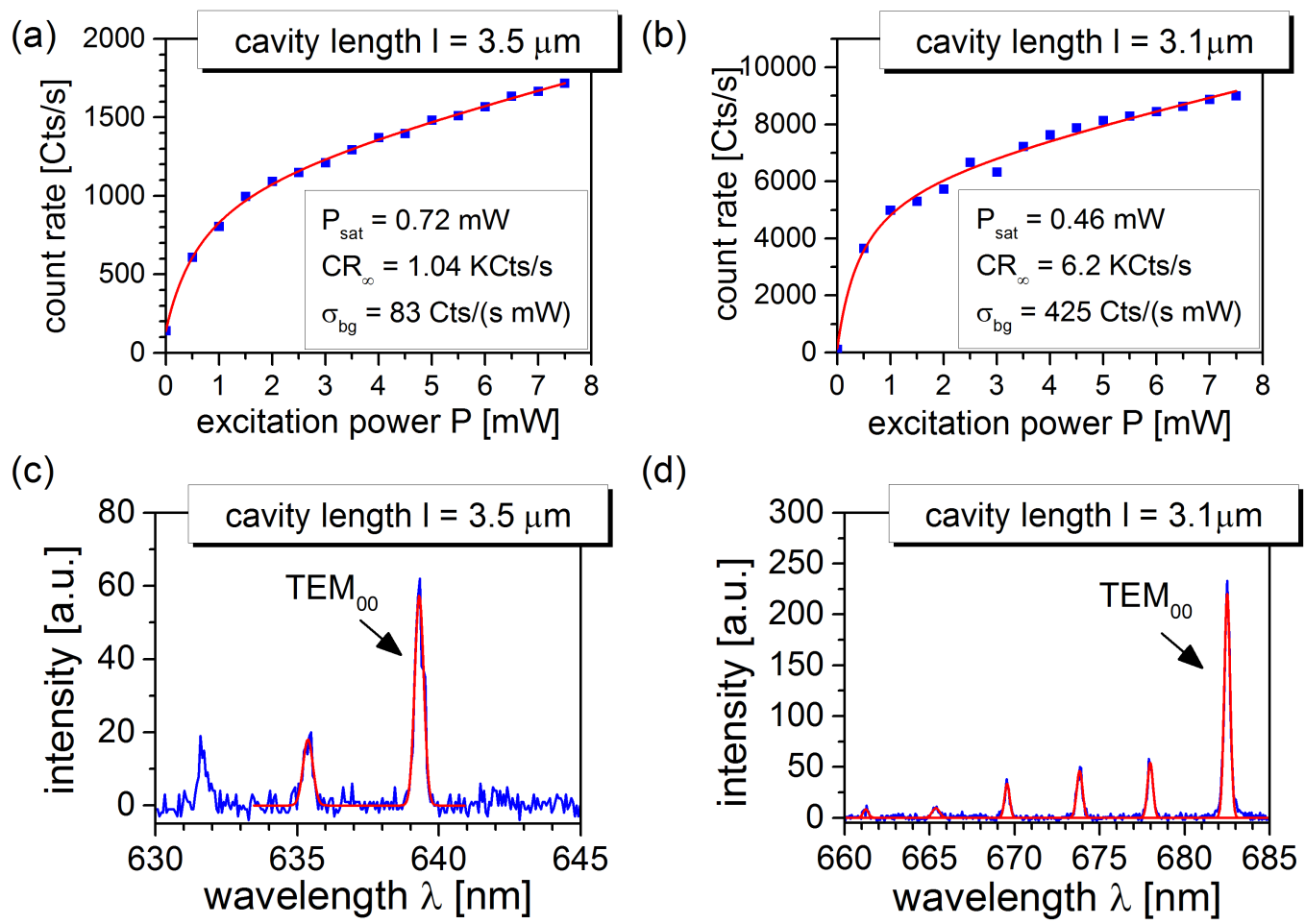

(d)

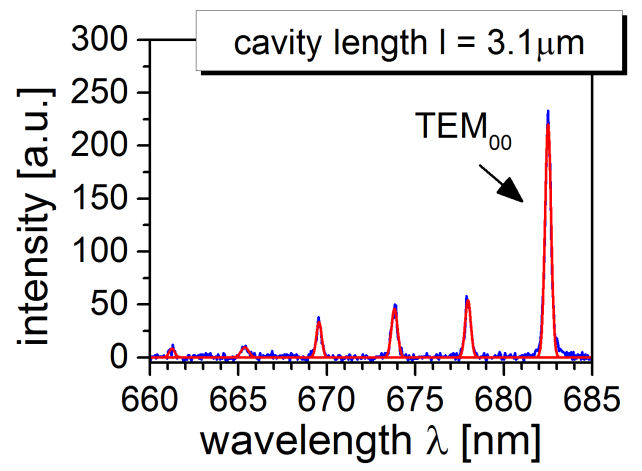

Figure 5.3: (a) Saturation measurement of the cavity coupled emission, where the wavelength of the cavity mode is in resonance with the ZPL. The power of the excitation laser is measured in front of the microscope objective. The obtained data are fitted with a saturation law according to Eq. 5.1 including a linear contribution from the background. The photons are detected with APDs in the spectral window $633-647 \mathrm{~nm}$. (b) Saturation measurement, where the wavelength of the cavity mode is at the maximum of the PSB emission. The spectral detection window is $650-750 \mathrm{~nm}$. The data are fitted as in (a). (c)\&(d) To determine the ratio of the detected photons that are within the fundamental $\mathrm{TEM}_{00}$ mode, the emission spectra of the cavity is fitted with several Gaussian peaks. For the two cases we obtain that $74 \%$ and $60 \%$ is contained within the fundamental mode. Note that in (c) only one of the higher transverse modes lies within the spectral detection window.

Note that this is also reflected in the line form: the resonance peaks shown in Fig. 5.3(c) and (d) are best fitted with Gaussians, as one expects for inhomogeneous broadening to which drifts in the cavity length belong to.

\subsubsection{Intensity correlation of the cavity emission}

From theoretical considerations, as described in Sect. 2.5, at room temperature we do not expect a change in lifetime of the NV center coupled to a fiber cavity with 
mode volume and finesse as are achieved here. The lifetime can be experimentally determined by time-correlated photon counting using a pulsed excitation laser. As shown above it is also accessible from measuring the intensity correlation function. Due to lack of a suitable pulsed laser we use the second method, which furthermore allows to demonstrate the non-classical character of the out-coupled light from the fiber cavity.

Figure 5.4(a) shows the intensity correlation function of the emission into free space at different excitation powers of the very same NV center, that is now coupled to the cavity. As described in Sect. 3.2.2 the measured data is fitted with $g^{(2)}(\tau)=$

(a)

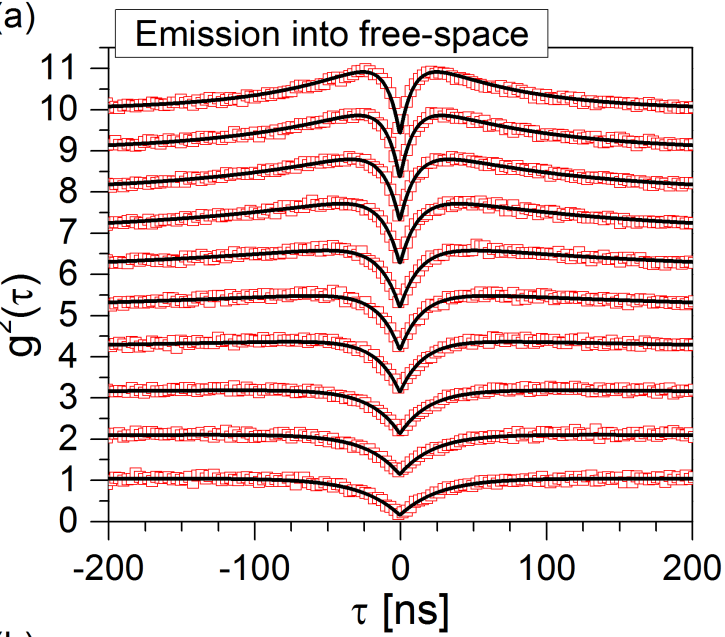

(b)

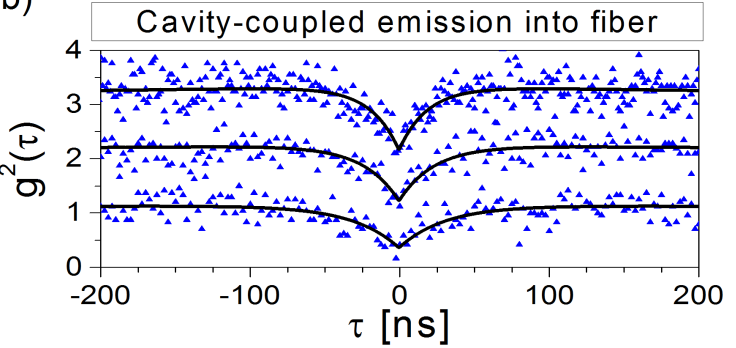

(c)

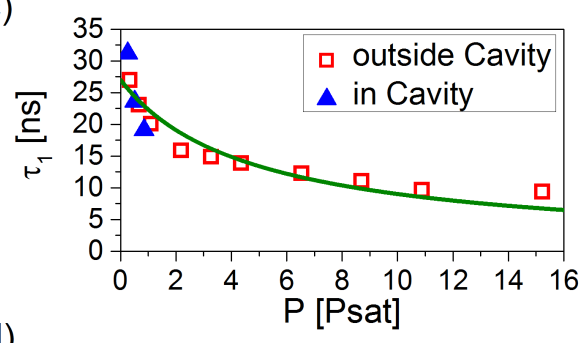

(d)

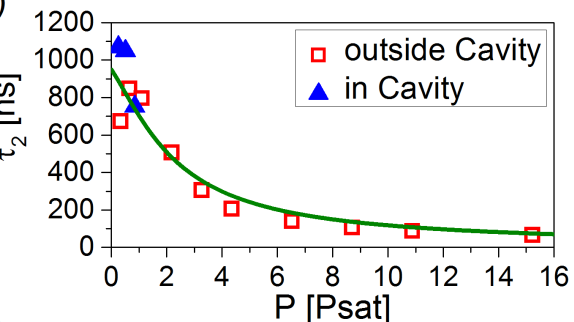

(e)

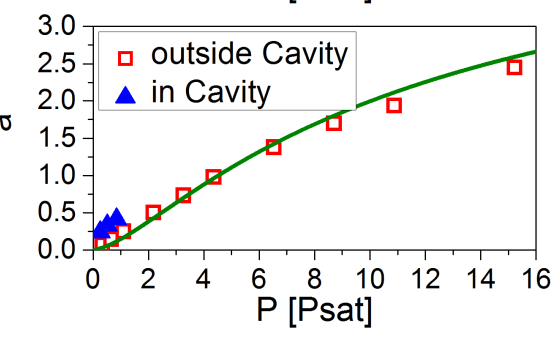

Figure 5.4: (a) Open squares: Intensity correlation $g^{(2)}(\tau)$ measurements at different excitation powers $P$ for a single NV center $(\# 41)$ on a plane mirror outside the cavity. The curves are shifted vertically by 1 each for better visibility. $P=0.32 \times, 0.65 \times, 1.1 \times, 2.2 \times$, $3.3 \times, 4.3 \times, 6.5 \times, 8.7 \times, 10.9 \times, 15.2 \times P_{\text {sat }}$ from bottom to top. Lines: Fit curves of the form $g^{(2)}(\tau)=1-(1+a) e^{-|\tau| / \tau_{1}}+a e^{-|\tau| / \tau_{2}}$. (b) Triangles: $g^{(2)}$ measurements at different excitation powers with the very same NV center as in (a) but inside the cavity. $P=0.25 \times, 0.51 \times$, $0.84 \times P_{\text {sat }}$ from bottom to top. Lines: Fit curves with the same function as in (a). (c) - (e) Squares and triangles: Model parameters $a, \tau_{1}, \tau_{2}$ obtained from the fits in (a) and (b). Solid line: Theoretical dependence of model parameters on excitation power. 
$1-(1+a) e^{-|\tau| / \tau_{1}}+a e^{-|\tau| / \tau_{2}}$. Figure 5.4(b) displays the measurements of the intensity correlation function of the emitted light from the cavity. The clear antibunching dip at $\tau=0$ proves that indeed a single NV center is coupled to the microcavity. The obtained fit parameters for both cases are summarized in Figs. 5.4(c)-(e). They agree within their margin of errors and thus, as expected, the internal dynamics is not modified by the cavity coupling. Remember that the lifetime of the excited state is given by $\lim _{P \rightarrow 0}\left(\tau_{1}\right)$.

\subsubsection{Cavity tuning over several free spectral ranges}

Fiber-based Fabry-Perot cavities are easily tunable. It suffices to change the cavity length via for instance a piezo-electric crystal. This allows to tune the cavity over several free spectral ranges. The voltage of the piezo actuator is changed in small steps of $0.2 \mathrm{~V}$, which corresponds to a change in cavity length of approximately $10 \mathrm{~nm}$. At each length the spectrum of the cavity emission is recorded. Lateral position and excitation of the NV center within the cavity are kept constant.

Figure 5.5 shows the color-coded spectra as function of cavity length which has been varied from $l=5.95 \mu \mathrm{m}$ down to $l=2.9 \mu \mathrm{m}$. Several longitudinal modes with mode number $n=17, \ldots, 9$ are shifted over the wavelength range of interest from $\lambda=630-700 \mathrm{~nm}$. Beside the fundamental $\mathrm{TEM}_{00}$ mode, higher transverse modes are also visible. Remember that the fundamental mode is lowest in frequency (cf. Eq. 2.44) and thus has the largest wavelength compared to higher transverse modes with the same longitudinal mode number $n$. The spectra shown before for a cavity of length $l=3.5 \mu \mathrm{m}$ and $l=3.1 \mu \mathrm{m}$ in Fig. 5.2 are simply the horizontal cross-sections of Fig. 5.5 at these cavity lengths.

The shift of the resonance wavelength depends on the mode number $n$. This is clearly visible in Fig. 5.5: the slope of cavity length verses resonance wavelength diminishes for smaller $n$. This is expected as we have $l=n \times \lambda / 2$ and thus $\Delta l=n \times \Delta \lambda / 2$.

Focusing on the mode with longitudinal mode number $n=9$, we recognize that below $l=2.95 \mu \mathrm{m}$ the rate of change in resonance wavelength is altered and does not follow the linear dependence as for longer cavities. At this point the edge of the fiber touches the plane mirror. As the fiber facet is not flat, it is still possible to diminish the cavity length by pushing the fiber against the mirror which results in bending of the fiber. However, this is not desirable and thus the practical limit for the smallest cavity length here is $2.95 \mu \mathrm{m}$. The slight non-linearity visible for the $n=17$ mode is due to the hysteresis of the piezo-electric crystal used to change the cavity length.

To analyze the spectra quantitatively we focus on the fundamental mode with a fixed longitudinal mode number $n$. They are summarized for $n=17, \ldots, 9$ into one plot in Fig. 5.6, where the TEM 00 modes belonging to the same $n$ have been cut out from the spectra at different cavity lengths and are plotted in one trace. The emitted photon rate is proportional to the area under each peak. We will compare this in the next section to the predictions of the theoretical model which enables, by fitting the measurement shown here, to determine the effective cavity round-trip losses, that, beside the losses of the mirror coatings, also include the scattering and absorption losses 


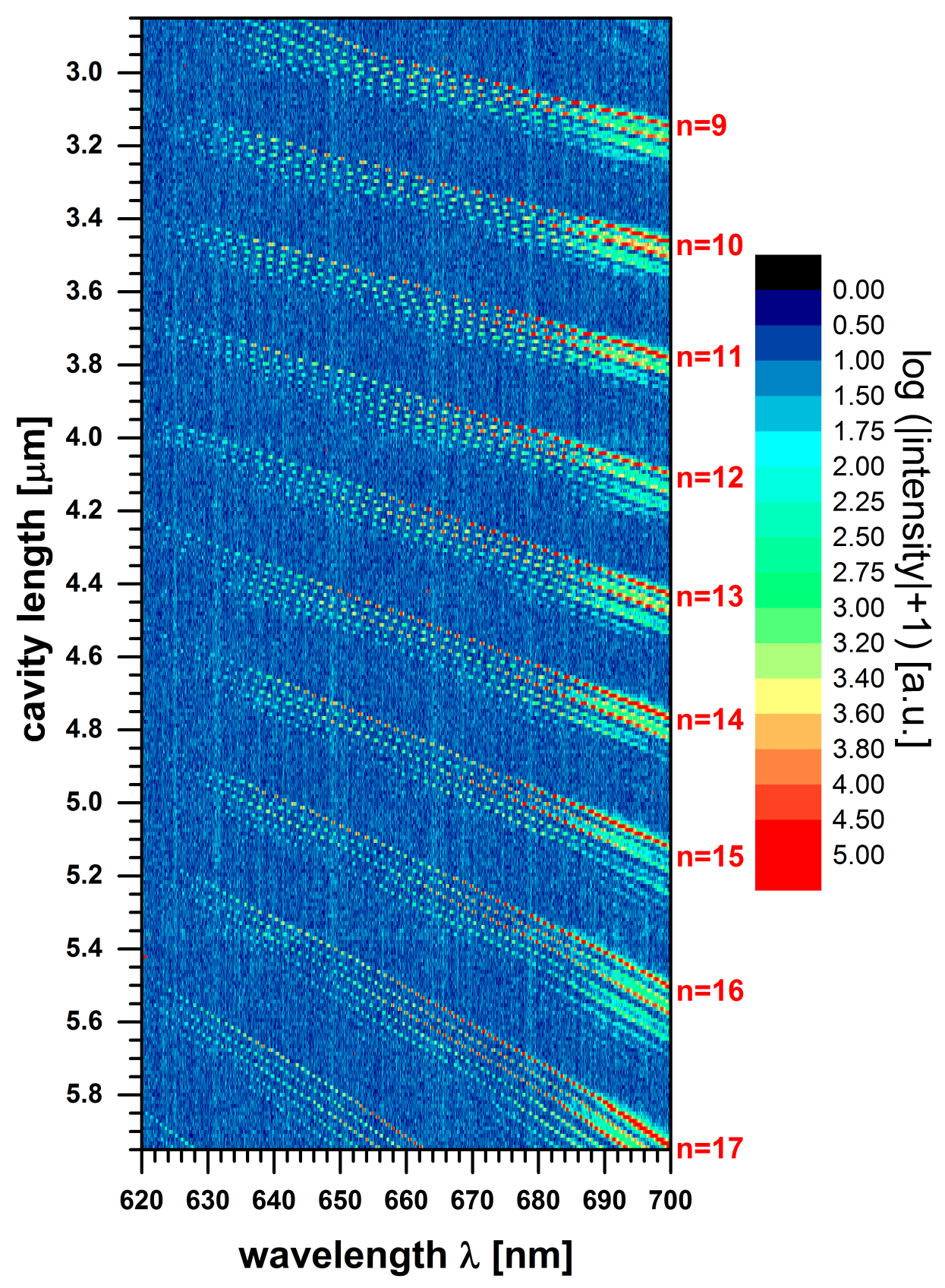

Figure 5.5: Consecutively recorded, color-coded emission spectra of the cavity using a logarithmic scale $[\log (\mid$ intensity $\mid+1)]$, such that the fundamental modes and higher transverse modes are visible. Between two consecutive spectra the cavity length is changed by $10 \mathrm{~nm}$. The longitudinal mode number is denoted by $n$. 


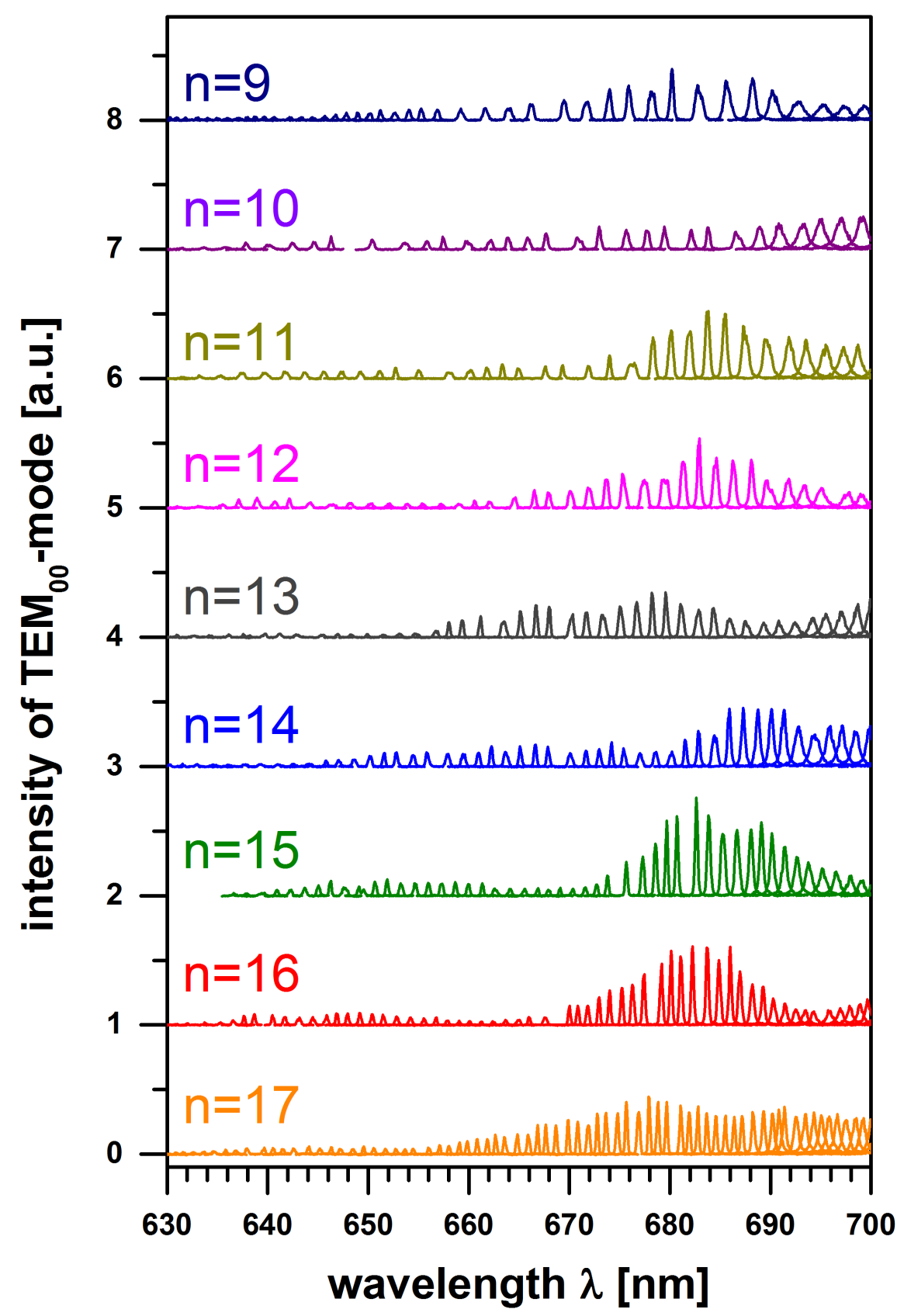

Figure 5.6: $\mathrm{TEM}_{00}$ modes cut-out from the emission spectra of Fig. 5.5 at different cavity lengths and plotted for a fixed longitudinal number $n$ in one trace. For better visibility the traces for different $n$ are vertically shifted by 1 . 
induced by the ND in the cavity waist.

As an important result, we have observed single photon emission into a narrow cavity mode over a very broad spectral range. The emission wavelength is controlled by varying the mirror separation. Thus, we have realized a continuously tunable, narrow band single photon source operating at room temperature!

\subsubsection{Comparison to theoretical predictions}

\section{Tuning spectra}

We will first evaluate the recorded spectra while tuning over several FSR. The number of photons emitted into the fundamental mode is proportional to the area under each peak in Fig. 5.6 denoted by $A_{\mathrm{TEM}_{00}}$ and is plotted in Fig. 5.7 (squares).

A clear modulation is recognizable. It originates from a change of the excitation intensity at the location of the NV center even though the power of the excitation laser is kept constant in front of the microscope objective that focuses it onto the ND through the plane mirror. Because of the high reflectivity of the coating on the fiber facet for the excitation wavelength at $532 \mathrm{~nm}$, there's for this wavelength a standing wave building up. Because the spot size of the focus is roughly the same as the cavity waist, the reflected beam from the fiber strongly interferes with the incoming beam. In case of constructive interference and perfect spatial overlap, the field intensity at the ND position is enhanced by a factor of 4 . Due to non-perfect mode matching we here observe a smaller factor: the count rate is modulated by a factor of the form $\left(0.5+A \sin \left(4 \pi l / \lambda_{\text {exc }}+\phi\right)\right)^{2}$ with $A$ the amplitude of the modulation and $\phi$ the phase offset between the standing wave at $\lambda_{\text {exc }}=532 \mathrm{~nm}$ and the resonant wave at the NV wavelength. Note that the effective cavity length for these two wavelengths is not the same due to the particular design of the coating on the fiber, i.e. two mirrors on top of each other.

We further have to take into account the out-coupling ratio into the fiber. Assuming a constant loss $L$ for all wavelengths (absorption and scattering in mirror coatings and ND), the overall losses within the cavity are $L+T_{\mathrm{f}}+T_{\mathrm{p}}$, where the transmission coefficients for the two mirror coatings, which are denoted by $T_{\mathrm{f}}$ for the fiber and $T_{\mathrm{p}}$ for the plane mirror, are wavelength-dependent. The ratio of the cavity field coupled out through the fiber mirror is given by

$$
\epsilon_{\text {out }}(\lambda)=\frac{T_{\mathrm{f}}(\lambda)}{L+T_{\mathrm{f}}(\lambda)+T_{\mathrm{p}}(\lambda)}
$$

Moreover, the spatial overlap between cavity mode and guided fiber mode, which is characterized by $\epsilon_{\mathrm{ov}}$ (see Sect. 4.2.4, Eq 4.10) further reduces the collected photon number. However, this is independent of the wavelength.

For a fixed longitudinal mode number $n$, where the cavity length is $l=n \times \lambda / 2$, we thus have

$$
A_{\mathrm{TEM}_{00}}(n, \lambda)=C \times \epsilon_{\mathrm{ov}} \times P_{\text {total }}(n, \lambda) \times \epsilon_{\text {out }}(\lambda) \times\left(0.5+A \sin \left(2 \pi n \lambda / \lambda_{\text {exc }}+\phi\right)\right)^{2},
$$




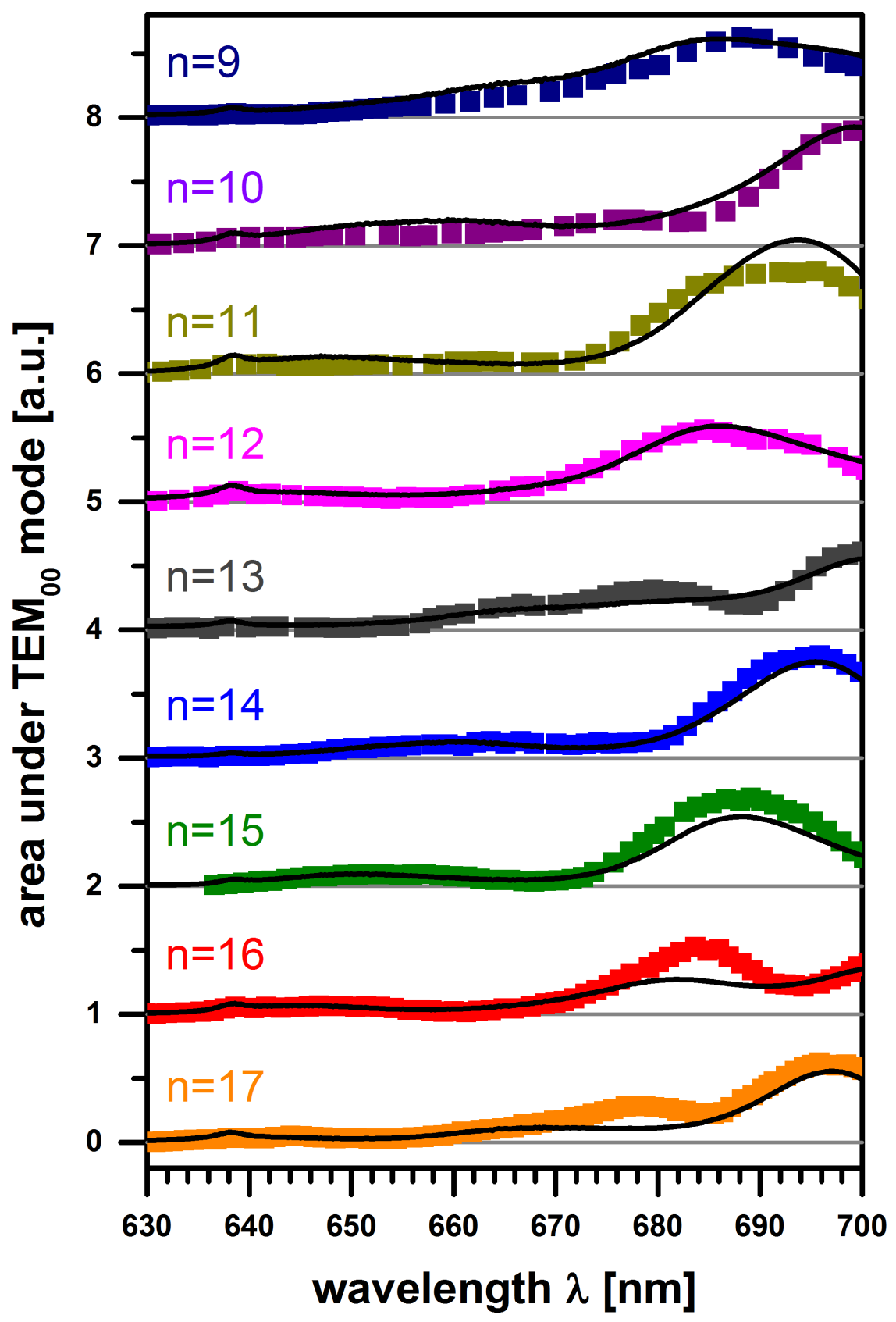

Figure 5.7: Sqares: Area $A_{\mathrm{TEM}_{00}}$ under each $\mathrm{TEM}_{00}$ mode plotted in Fig. 5.6 which is proportional to the emitted photon rate from the cavity. Solid lines: Theoretical dependence of the emission rate as function of cavity resonance wavelength $\lambda$ for different longitudinal mode numbers $n$ predicted by the rate model. 
where $P_{\text {total }}(n, \lambda)$ is the theoretically expected cavity-enhanced emission of the NV center into the cavity mode as described earlier (see Sect. 2.4.2 Eq. 2.85). We fit the measurements for several different longitudinal mode numbers $(n=9 \ldots 17)$ simultaneously to determine the proportionality factor $\mathrm{C}$, the unknown parameters $A$ and $\phi$ of the modulation and the total round-trip losses $L$. From the fit we obtain round-trip losses $L=0.54 \%, A=0.12$ and $\phi=-0.36$. The obtained fit curves are plotted as solid lines in Fig. 5.7. The obtained losses are rather large. However, they are similar to the observed losses in preliminary experiments (not explicitly shown) where a densely spin-coated mirror was used.

The theoretical curves well reproduce the measurements. Especially the increase in count rate due to the stronger coupling rate $g$ for smaller cavities is confirmed. To obtain a more quantitatively comparison we focus next on the two cases described above with a cavity length of $3.5 \mu \mathrm{m}$ and $3.1 \mu \mathrm{m}$, where we determined the saturation photon rate with APDs.

\section{Collection efficiency}

For a quantitative analysis we first need to determine the spatial collection efficiency that we achieve when detecting the emission into free space. We perform a numerical simulation of the Maxwell equations for a emitting dipole $30 \mathrm{~nm}$ above a dielectric plane mirror based on finite-difference-time-domain (FDTD) method using a commercial software (Lumerical, FDTD solutions). The exact structure of the plane mirror that we use here is known, i.e. the exact thicknesses of the alternating dielectric layers. The available computational capability only allows for $2 \mathrm{D}$ simulations. We assume the dipole to be parallel to the plane mirror. This is justified from experimental bias, because we have chosen to investigate only bright emitters, whose orientation needs to be close to this ideal case. We simulate two configurations: first, where the dipole lies within the simulation-plane (TE), and second, where the dipole is perpendicular to the simulation-plane (TM). The emitted power is recorded at each side (right, top, left, bottom) with four monitors. The simulation cell is illustrated in Fig. 5.8(a). The transmitted power through the four monitors for both cases (TE and TM) are shown in Fig 5.8(b) and (c). A large part of the emission is upward. The emission through the mirror is considerably less. However, as seen on the side monitors, a significant part of the emission is transmitted within the layers towards the side of the mirror. The layers with the low refractive index $\left(\mathrm{SiO}_{2}, n_{L}=1.455\right)$ imbedded within the layers with the high refractive index $\left(\mathrm{Ta}_{2} \mathrm{O}_{5}, n_{H}=2.041\right)$ form a waveguide for rays with $\mathrm{k}$-vectors having a large angle of incidence (with respect to the normal of the mirror). The critical angle for total internal reflection for the two refractive indices of $\mathrm{SiO}_{2}$ and $\mathrm{Ta}_{2} \mathrm{O}_{5}$ is $\theta_{\text {tir }}=45.5^{\circ}$.

To characterize the emission into free space, a microscope objective with a N.A. of 0.8 was used which corresponds to a collection angle of $53.1^{\circ}$. Averaging over the two orientations for the dipole (TE and TM) we obtain a collection efficiency of $42 \%$.

One of the inaccuracies of the simulation is the limited size of the mirror substrate. 
(a)

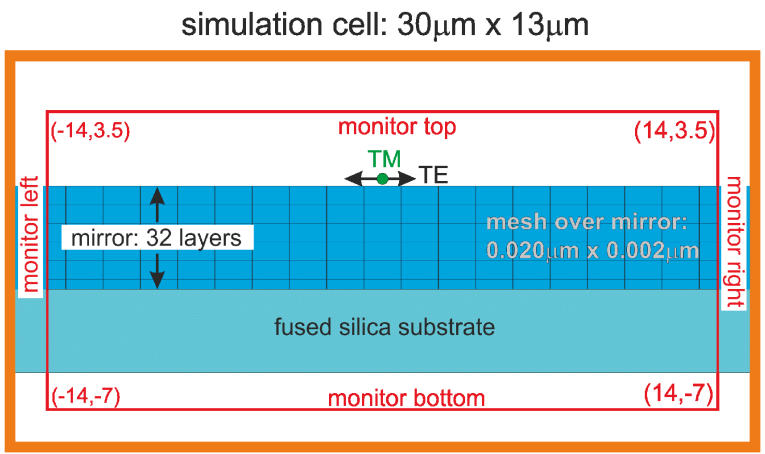

(b)
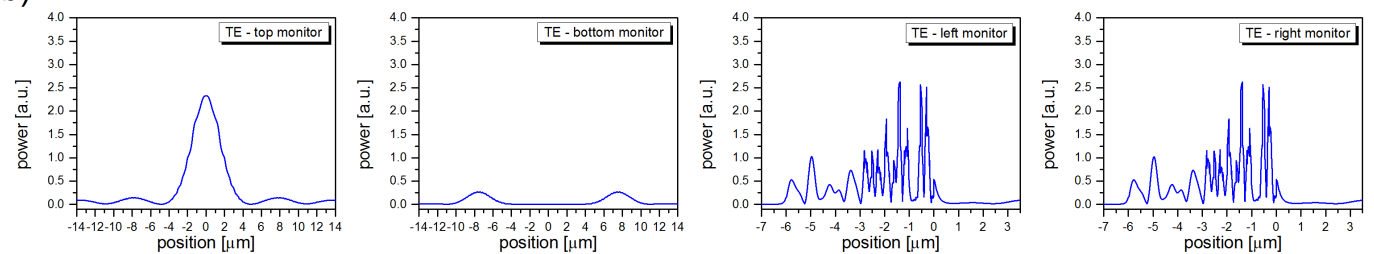

(c)
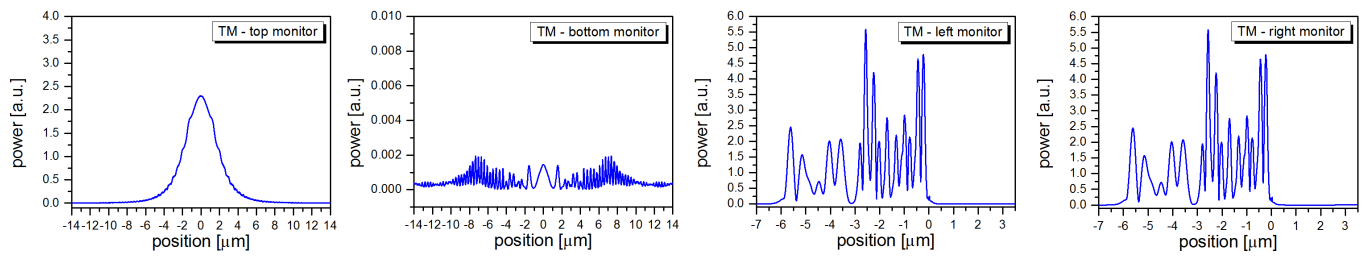

Figure 5.8: (a) Simulation cell for a 2D-FDTD-calculation to determine the collection efficiency from a NV center on a mirror. The parameters of the mirror, i.e. the thickness of the 32 alternating dielectric layers on top of a $\mathrm{SiO}_{2}$ substrate, correspond exactly to ones of the mirror used in the experiment. Four monitors, one on each side, measure the transmitted power. The emitting dipole is assumed to be parallel to the mirror. Two configurations are simulated: first, where the dipole lies within the simulation-plane (TE), and second, where the dipole is perpendicular to the simulation-plane (TM). (b) and (c) transmitted power through the four monitors for the two cases of a TE and TM oriented dipole.

This also leads to a standing wave pattern within the substrate which does not reflect the situation in the real mirror. Further, we have only considered a single dipole emitting at the NV ZPL wavelength which is a strong simplification of the full characteristics of the NV center. It is worth noting, that the experimentally obtained ratio of the emission within the ZPL from NV centers in a ND on the mirror (see table 3.2) agree with the Huang-Rhys factor reported in literature. Thus, the emission spectrum is not significantly altered by the mirror. Concerning the obtained absolute number for collection efficiency some caution is necessary: On a glass substrate approximately $40 \%$ is emitted towards free space and $60 \%$ into the substrate due to higher density of states in glass attributed to the higher refractive index compared to air. Assuming a dipole 
emission pattern, where the dipole lies parallel to the interface, a microscope objective with N.A. 0.8 roughly collects $50 \%$ of the emission into free space [212], hence $20 \%$ of the total emission. Thus, the obtained collection efficiency of $42 \%$ for the case of a dipole on a mirror seems rather high.

\section{Cavity acting as filter}

It is instructive to compare the measured saturation count rates to the ones expected in the case, where the cavity acts only as spectral and spatial filter and no coupling occurs. To remember, we have measured the following saturation count rates:

$$
\begin{aligned}
\mathrm{CR}_{\infty}, \text { into free space }(\lambda=650-750 \mathrm{~nm}) & =2.9 \times 10^{5} \text { Counts } / \mathrm{s} \\
\operatorname{CR}_{\infty}, \text { cavity-coupled }(\lambda=639.3 \mathrm{~nm}, n=11) & =770 \text { Counts } / \mathrm{s} \\
\mathrm{CR}_{\infty}, \text { cavity-coupled }(\lambda=682.5 \mathrm{~nm}, n=9) & =3700 \text { Counts } / \mathrm{s}
\end{aligned}
$$

In the case the cavity acts as filter, we have to consider spatial and spectral filtering, taking into account the collection solid angle of cavity mode and fiber, the cavity linewidth and output coupling efficiency. The spectral bandwidth for emission into free space is given by the bandpass filters $(650-750 \mathrm{~nm})$ employed, whereas for the cavity it is determined by the finesse $\mathcal{F}$ and FSR: $\delta \nu=\mathrm{FSR} / \mathcal{F}=\operatorname{FSR}\left(T_{f}+T_{p}+L\right) /(2 \pi)$, where $T_{f}\left(T_{p}\right)$ are the transmission coefficients of the coating on the fiber (plane mirror) and $L$ the round-trip losses of the cavity. The spatial filtering arises from the difference in collection angle between microscope objective $(N . A .=0.8)$ and fiber $(N . A .=0.12)$. Furthermore, we have to take into account the out-coupling ratio of the cavity through the fiber mirror $\epsilon_{\text {out }}(\lambda)$ (cf. Eq. 5.2) and the finite spatial overlap between cavity mode and guided fiber mode $\epsilon_{\mathrm{ov}}$ (cf. Eq. 4.10). Table 5.1 compares all these factors for emission into free space with the cavity case.

\begin{tabular}{|c|c|c|c|}
\hline \hline & free space & cavity-coupled & ratio cavity/free space \\
\hline numerical aperture & $N A=0.8$ & $\mathrm{NA}=0.12$ & \\
\hline spatial collection efficiency & $42 \%$ & $8.5 \%$ & 0.2 \\
\hline collection bandwidth & $62 \mathrm{THz}$ & $46 \mathrm{GHz}(90 \mathrm{GHz})$ & $7 \times 10^{-4}\left(1.4 \times 10^{-3}\right)$ \\
\hline$\epsilon_{\text {out }}$ & 1 & $0.18(0.44)$ & $0.18(0.44)$ \\
\hline$\epsilon_{\text {ov }}$ & 1 & 0.26 & 0.26 \\
\hline Total & & & $7 \times 10^{-6}\left(3 \times 10^{-5}\right)$ \\
\hline \hline
\end{tabular}

Table 5.1: List of relevant factors contributing to spatially and spectrally filtering of the NV center emission for a cavity with resonance wavelength $\lambda_{\text {cavity }}=640 \mathrm{~nm}\left(\lambda_{\text {cavity }}=682 \mathrm{~nm}\right)$. In cases, where the values for ZPL and PSB are the same, only one value is specified. We have taken into account the above determined cavity round trip losses $L=0.5 \%$. 
For a cavity in resonance with the ZPL we expect that a factor of $7 \times 10^{-6}$ of the NV center emission is transmitted into the guided fiber mode. In free space we observe a saturation intensity of $2.9 \times 10^{5}$ Counts/s. Thus filtering by the cavity leads to a saturation count rate of $\approx 2$ photons/s. This is more than 2 orders of magnitude lower than the observed saturation count rates. Similar for a cavity with resonance wavelength close the maximum of the PSB $(\lambda=682 \mathrm{~nm})$ spatial and spectral filtering would result in a photon saturation count rate of 9 photons/s. Again this is more than 2 orders of magnitude lower than the observed saturation count rates.

It is worth mentioning that already when looking at the spectral photon density of the observed cavity emission, an enhancement can be recognized. The cavity linewidth at the wavelengths $\lambda_{\mathrm{ZPL}}=640 \mathrm{~nm}\left(\lambda_{\mathrm{PSB}}=680 \mathrm{~nm}\right)$ is $\delta \nu=46 \mathrm{GHz}(\delta \nu=90 \mathrm{GHz})$ taking into account the observed cavity round trip losses of $L=0.5 \%$. Thus the observed spectral photon density at these wavelengths is 17 (41) photons/(s GHz) which is significantly larger than the value of 4.7 photons/(s GHz) observed for the emission into free space.

In conclusion, the observed emission rates into the cavity can only be explained by a coupling of the emitter to the cavity beyond simple filtering effects. Quantitative comparison in the next section will confirm that this coupling is well described by the theoretical description presented in Sect. 2.4.

\section{Quantitative comparison}

We now calculate from the rate model the theoretically expected saturation count rates and compare them to the observed values. As described in Sect. 3.3 fitting of the emission spectrum with several Lorentzians yields the necessary input parameters for the model, i.e. the energies of the vibrational ground state levels $E_{i}$ and excited state $E_{e}$, the pure dephasing rate $\gamma^{\star}$ and the fast ground state relaxation rates $\gamma_{i, i-1}$. We further determine the spontaneous emission rates $\gamma_{i}$ and the coherent coupling rates via

$$
\begin{aligned}
& \gamma_{i}=A_{\text {rel }} \times \gamma_{\mathrm{tot}} \\
& g_{i}=\sqrt{A_{\text {rel }}} \times g, \quad i=0, \ldots, 7 \quad \text { where } g=\sqrt{\frac{3 c \lambda^{2} \gamma_{\mathrm{tot}} / 2}{4 \pi V_{\text {mod }}},}
\end{aligned}
$$

where $A_{\text {rel }}=A_{i} / \sum_{j} A_{j}$ is the relative area of the i-th transition as obtained from the fit and $\gamma_{\text {tot }}$ is the total optical decay rate of the emitter, that has been inferred from measurements of the intensity correlation function.

We then use Eqs. 2.83 to determine the diffusion rates $R_{i}$ and Eqs. 2.84 to calculate the efficiency $P_{i}$ of the ZPL and each of the PSB to decay into the cavity mode. For the two cases investigated in the experiment: the obtained values and all input parameters are listed in table 5.2 for the case of a cavity with effective length $l=3.5 \mu \mathrm{m}$ and 


\begin{tabular}{|c|c|c|c|c|c|c|c|}
\hline $\mathrm{i}$ & $\begin{array}{c}E_{i} \\
{[\mathrm{meV}]}\end{array}$ & $\begin{array}{c}\gamma_{i} \\
{[\mathrm{MHz}]}\end{array}$ & $\begin{array}{c}\gamma^{\star} \\
{[\mathrm{THz}]}\end{array}$ & $\begin{array}{c}\gamma_{i, i-1} \\
{[\mathrm{THz}]}\end{array}$ & $\begin{array}{c}g_{i} \\
{[\mathrm{GHz}]}\end{array}$ & $\begin{array}{c}R_{i} \\
{[\mathrm{KHz}]}\end{array}$ & $\begin{array}{c}P_{i} \\
{[\%]}\end{array}$ \\
\hline \hline 0 & 0 & 0.74 & 15.3 & - & 1.1 & 220 & 0.60 \\
1 & 31.8 & 2.58 & 15.3 & 85 & 2.1 & 99 & 0.27 \\
2 & 70.3 & 9.14 & 15.3 & 82 & 3.9 & 120 & 0.32 \\
3 & 124 & 8.07 & 15.3 & 79 & 3.7 & 36 & 0.096 \\
4 & 168 & 6.89 & 15.3 & 88 & 3.4 & 18 & 0.049 \\
5 & 221 & 4.52 & 15.3 & 65 & 2.8 & 5.5 & 0.015 \\
6 & 275 & 3.23 & 15.3 & 71 & 2.3 & 2.7 & 0.0073 \\
7 & 319 & 1.61 & 15.3 & 86 & 1.6 & 1.2 & 0.0032 \\
\hline $\mathrm{e}$ & 1941 & - & - & - & - & $P_{\text {tot }}:$ & 1.35 \\
\hline \hline
\end{tabular}

Table 5.2: Full list of parameters for the rate model and the obtained results for the case of a cavity with effective length $l=3.5 \mu \mathrm{m}$ and resonance frequency at $\lambda_{\text {cavity }}=639.3 \mathrm{~nm}$.

resonance wavelength of $\lambda_{\text {cavity }}=639.3 \mathrm{~nm}$ and in table 5.3 for the case of a cavity with effective length $l=3.1 \mu \mathrm{m}$ and resonance wavelength $\lambda_{\text {cavity }}=682.5 \mathrm{~nm}$.

\begin{tabular}{|c|c|c|c|c|c|c|c|}
\hline \hline $\mathrm{i}$ & $\begin{array}{c}E_{i} / \hbar \\
{[\mathrm{meV}]}\end{array}$ & $\begin{array}{c}\gamma_{i} \\
{[\mathrm{MHz}]}\end{array}$ & $\begin{array}{c}\gamma^{\star} \\
{[\mathrm{THz}]}\end{array}$ & $\begin{array}{c}\gamma_{i, i-1} \\
{[\mathrm{THz}]}\end{array}$ & $\begin{array}{c}g_{i} \\
{[\mathrm{GHz}]}\end{array}$ & $\begin{array}{c}R_{i} \\
{[\mathrm{KHz}]}\end{array}$ & $\begin{array}{c}P_{i} \\
{[\%]}\end{array}$ \\
\hline 0 & 0 & 0.74 & 15.3 & - & 1.3 & 0.68 & 0.0018 \\
1 & 31.8 & 2.58 & 15.3 & 85 & 2.4 & 25 & 0.064 \\
2 & 70.3 & 9.14 & 15.3 & 82 & 4.5 & 200 & 0.54 \\
3 & 124 & 8.07 & 15.3 & 79 & 4.2 & 750 & 1.96 \\
4 & 168 & 6.89 & 15.3 & 88 & 3.9 & 230 & 0.61 \\
5 & 221 & 4.52 & 15.3 & 65 & 3.1 & 35 & 0.093 \\
6 & 275 & 3.23 & 15.3 & 71 & 2.7 & 12 & 0.031 \\
7 & 319 & 1.61 & 15.3 & 86 & 1.9 & 4.0 & 0.011 \\
\hline $\mathrm{e}$ & 1941 & - & - & - & - & $P_{\text {tot }}:$ & 3.31 \\
\hline \hline
\end{tabular}

Table 5.3: Full list of parameters for the rate model and the obtained results for the case of a cavity with effective length $l=3.1 \mu \mathrm{m}$ and resonance frequency at $\lambda_{\text {cavity }}=682.5 \mathrm{~nm}$

To gain absolute numbers for photon rates emitted from the cavity we estimate the NV center's total free-space emission rate as $2.9 \times 10^{5} /(0.7 \times 0.42)$ photons/s to account for the collection efficiency ( $42 \%$ ) of the microscope objective and the fact that $70 \%$ of the NV emission lies within the detected spectral interval $(650-750 \mathrm{~nm})$ (determined from the NV emission spectrum). The out-coupling from the cavity through the fiber- 
mirror is governed by $\epsilon_{\text {out }}$ and the spatial overlap between the cavity mode and the guided fiber mode $\epsilon_{\mathrm{ov}}$. The numbers are summarized in table 5.4.

\begin{tabular}{|c|c|c|c|c|c|c|c|c|c|c|}
\hline \hline $\begin{array}{c}l \\
{[\mathrm{~nm}]}\end{array}$ & $\begin{array}{c}\lambda \\
{[\mathrm{nm}]}\end{array}$ & $\begin{array}{c}T_{f}(\lambda) \\
{[\%]}\end{array}$ & $\begin{array}{c}T_{p}(\lambda) \\
{[\%]}\end{array}$ & $\begin{array}{c}L \\
{[\%]}\end{array}$ & $\mathcal{F}$ & $\begin{array}{c}\delta \nu \\
{[\mathrm{GHz}]}\end{array}$ & $\begin{array}{c}\kappa \\
{[\mathrm{GHz}]}\end{array}$ & $\begin{array}{c}V_{\text {mod }} \\
{\left[\lambda^{3}\right]}\end{array}$ & $\begin{array}{c}\epsilon_{\text {out }} \\
{[\%]}\end{array}$ & $\begin{array}{c}\epsilon_{\text {ov }} \\
{[\%]}\end{array}$ \\
\hline 3.5 & 639.3 & 0.12 & 0.007 & 0.54 & 940 & 46 & 286 & 32.7 & 18.0 & 26 \\
3.1 & 682.5 & 0.52 & 0.11 & 0.54 & 540 & 90 & 566 & 24 & 44.4 & 26 \\
\hline \hline
\end{tabular}

Table 5.4: Full list of parameters of the cavity with length $l=3.5 \mu \mathrm{m}(l=3.1 \mu \mathrm{m})$ and resonance frequency at $\lambda_{\text {cavity }}=639.3 \mathrm{~nm}\left(\lambda_{\text {cavity }}=685.2 \mathrm{~nm}\right) . \quad T_{f}(\lambda)\left(T_{p}(\lambda)\right)$ denote the transmission of the coating on the fiber (plane mirror), $L$ the round-trip losses of the cavity, $\mathcal{F}$ the finesse, $\delta \nu$ the spectral bandwidth, $\boldsymbol{\kappa}$ the decay-rate of the cavity and $V_{\text {mod }}$ the mode volume of the cavity.

Taking all these values into account we obtain the following saturation count rates predicted by the rate model:

$$
\begin{aligned}
& \mathrm{CR}_{\infty, \text { theo }}(\lambda=639.6 \mathrm{~nm}, n=11)=620 \text { Counts } / \mathrm{s} \\
& \mathrm{CR}_{\infty} \text {, theo }(\lambda=682.5 \mathrm{~nm}, n=9)=3700 \text { Counts } / \mathrm{s} \text {. }
\end{aligned}
$$

These values are in very good agreement with the measured values (see Eqs. 5.4)! Hence, we have clearly demonstrated coupling of a single NV center to a microcavity beyond simple filtering effects. Usually the NV center's broad emission bandwidth is considered as drawback. In the coupling regime here the broad emission bandwidth is used as resource for cavity coupling. The emission into the cavity is due to channeling of an off-resonant broad emitter into the narrow cavity mode mediated by pure dephasing. Our system is an ideal test bench to study this phonon-assisted coupling regime, because we can investigate both the emission into free space and the cavity-coupled emission and furthermore, by varying the cavity length, we can continuously vary the coherent coupling rate $g$. It is also worth mentioning that this coupling regime has previously not been considered for the NV center in diamond and first been published by us [174]. 


\subsection{Single nitrogen-vacancy center coupled to a fiber-fiber cavity}

In this section we discuss the results obtained from a ND incorporated in a fiber-fiber cavity. For this a ND containing a single NV center has been deposited in the center of spherical imprint on a fiber facet by the AFM pick and place method described in [79]. The placement has been performed in collaboration with the group of Oliver Benson at Humboldt-University, Berlin. Prior to transferring the ND, the presence of only a single NV center hosted in the ND has been verified. However, the emission spectrum had not been recorded and no saturation measurement had been performed. Furthermore, the $g^{(2)}$-function has only been recorded at one specific power.

In a first step the NV center's emission is characterized via collecting the fluorescence with a microscope objective and excitation of the NV center through the fiber. In a second step the coupling to the fiber-fiber cavity is investigated.

The fiber, onto which the diamond is deposited, is a pure silica core SM fiber (Nufern, S-460HP) with a MFD $\omega_{\text {fiber }}=3.5 \mu \mathrm{m}$. A spherical imprint has been fabricated via FIB machining with diameter $D=11 \mu \mathrm{m}$, depth $h=1.2 \mu \mathrm{m}$ and radius of curvature $R=14.2 \mu \mathrm{m}$. The fiber has the "mirror 1" dielectric coating (see Sect. 4.2.2), that is highly reflective at the ZPL wavelength $(\mathrm{R}=99.995 \%$ at $\lambda=638 \mathrm{~nm})$ and anti-reflective coated for the excitation wavelength $(T>95 \%$ at $\lambda=532 \mathrm{~nm})$. This fiber will be called henceforth "excitation fiber".

(a)

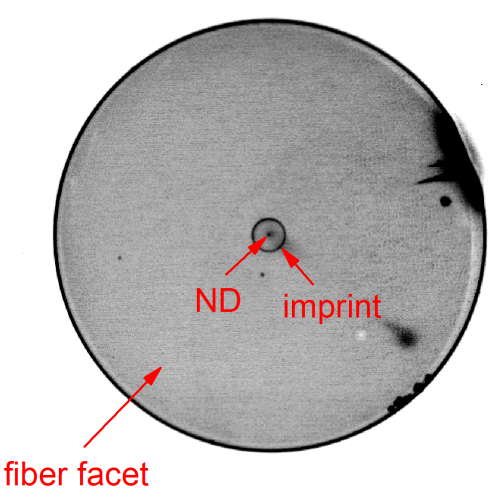

(b)

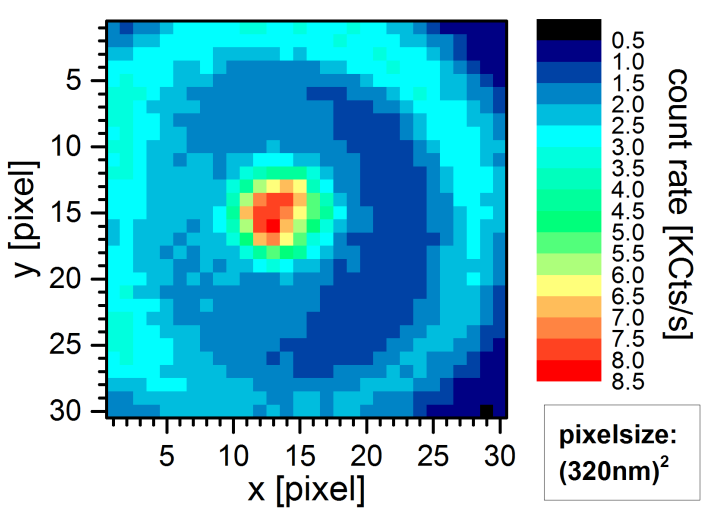

Figure 5.9: (a) Microscope image of the fiber mirror with a ND deposited in its center. The excitation laser at a wavelength $\lambda=532 \mathrm{~nm}$ is coupled into the fiber from its other end. The facet is imaged with a microscope objective (Olympus LMPLFLN, magnification 100x, N.A. 0.8, focal length $\mathrm{f}=1.8 \mathrm{~mm}$, working distance $3.4 \mathrm{~mm}$ ) onto a CCD camera. The excitation laser is blocked in front of the camera with a dielectric bandpass filter $(640 \pm 7 \mathrm{~nm})$. (b) Scanning confocal image of the center of the excitation fiber mirror showing the spherical imprint and the NV center in its center. The emission has been filtered spectrally to $633-647 \mathrm{~nm}$. 
A microscope objective (Olympus 100x, N.A. 0.8) is mounted in front of the fiber mirror with the ND. The excitation laser is coupled into the excitation fiber from its other end. The collected light is first imaged onto a CCD camera. With a dielectric bandpass filter $(640 \pm 7 \mathrm{~nm})$ the excitation laser is blocked in front of the camera. Figure 5.9(a) shows the obtained inverted image. The spherical imprint in the center of the fiber is clearly recognizable due to scattering at its edge. In its center there's a bright spot that corresponds to the fluorescence from the single NV center in the ND, that has been deposited on the excitation fiber facet.

In order to obtain a scanning confocal image of the fiber mirror, the collected light by the microscope objective is focused with a achromatic doublet lens $(\mathrm{f}=50 \mathrm{~mm})$ onto a MM-fiber (core diameter $50 \mu \mathrm{m}$ ) as illustrated in Fig. 5.10(a). A longpass filter is employed to block the excitation laser (3rd 620LP). The fluorescence rate is recorded with a single APD (MPD PDM PD5CTC). A lateral scan of the excitation fiber yields the confocal image as shown in Fig. 5.9(b). To measure saturation intensity and $g^{(2)}$ function as well as the emission spectrum the fiber is connected to a HBT-setup (for details see B) or a spectrometer (for details see B), respectively.

The emission spectrum is shown in Fig. 5.10(b). At $\approx 640 \mathrm{~nm}$ the ZPL of a NV center is recognizable. At longer wavelengths the background fluorescence generated within the fiber by the excitation laser dominates the spectrum because it is not blocked by the mirror coating on the fiber facet, which is highly reflective for the ZPL wavelength but becomes transparent for wavelengths $>680 \mathrm{~nm}$ (see Fig. 4.11). Filtering only the emission around the ZPL $(640 \pm 7 \mathrm{~nm})$ allows to perform a saturation measurement as displayed in Fig. 5.10(c), yielding a saturation count rate of $\mathrm{CR}_{\infty}=41800$ Counts $/ \mathrm{s}$ and a saturation power of $P_{\text {sat }}=12.6 \mathrm{~mW}$. The power of the excitation laser has been measured directly after the fiber without collimation optics. As the size of the excitation spot is defined by the MFD of the fiber $(3.5 \mu \mathrm{m})$, which is roughly a factor of 4 larger compared to the case using a microscope objective, the saturation power is correspondingly higher: In experiments shown in Sect 3.2, where we have investigated single NV centers on a plane mirror, that have been excited through the plane mirror with an microscope objective, we have measured saturation powers between $0.5-0.9 \mathrm{~mW}$. The focus size was roughly $0.9 \mu \mathrm{m}$. The linear background has been inferred from the measurement of the intensity correlation function $g^{(2)}$ shown in Fig. 5.10(d). As prior to deposition of the diamond on the fiber, the presence of only a single NV center has been verified, the SNR can be determined from the $g^{(2)}(\tau=0)$ value differing from zero (cf. Eq. 3.3). At an excitation power of $P=2.5 \mathrm{~mW}$ we have $g^{(2)}(0)=0.52$ and thus we obtain SNR $=2.2$. Assuming a typical NV spectrum, approximately $5.5 \%$ of the emission lies within the bandwidth from $633-647 \mathrm{~nm}$. As described above we collect $42 \%$ of the emission with the N.A. $=0.8$ microscope objective. Thus the total emission rate of the NV center is:

$$
\mathrm{CR}_{\infty}, \text { into-free-space }=1.8 \times 10^{6} \text { Counts } / \mathrm{s}
$$

This is a typical value for a NV center and on the same order of magnitude as for all NV centers discussed in Sect. 3.2. 
(a)

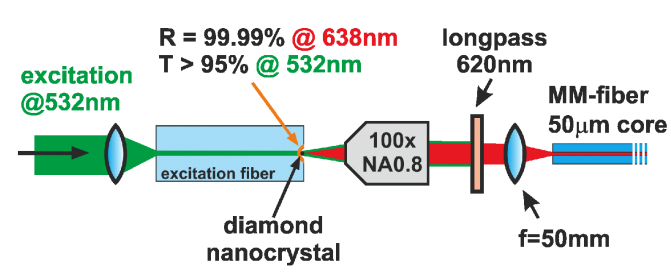

(c)

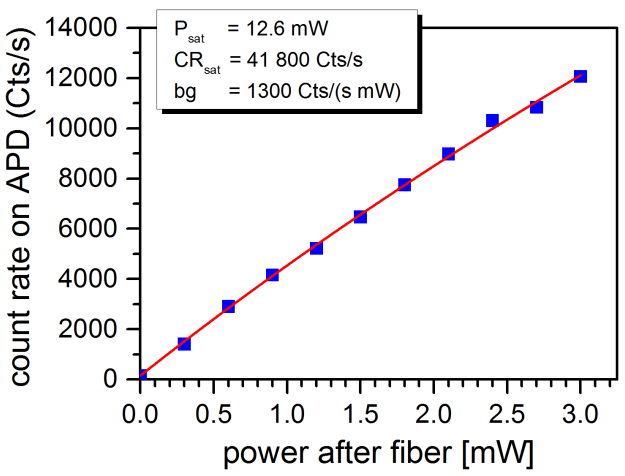

(b)

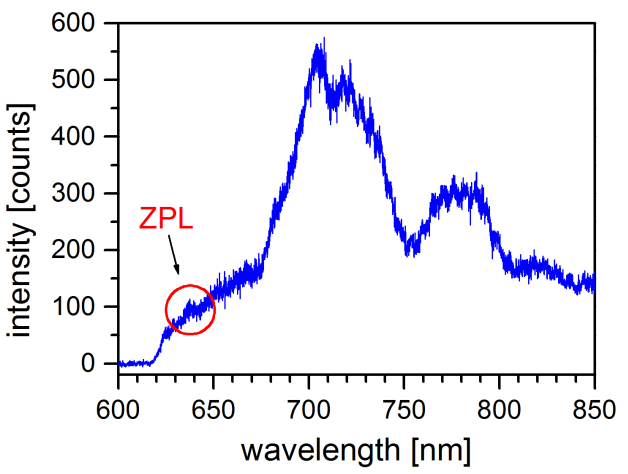

(d)

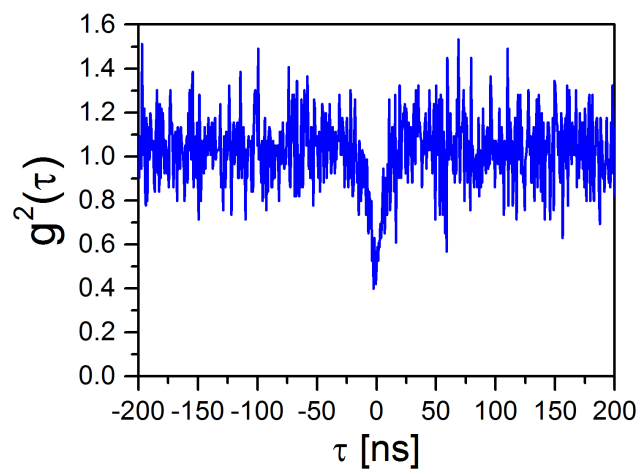

Figure 5.10: (a) Setup to measure the free space emission of a NV center in a ND, that has been deposited on a fiber facet. The excitation laser is coupled in through the fiber. The fluorescence is collected with a microscope objective (Olympus 100x, N.A. 0.8) in front of the fiber facet and to obtain a confocal setup focused onto a $50 \mu \mathrm{m}$ core of a MM fiber with a $f=50 \mathrm{~mm}$ aspheric lens. The excitation laser is filtered out with a longpass filter with transmission edge at $620 \mathrm{~nm}$. (b) Emission spectrum into free space. Note that dielectric mirror coating on the facet becomes transparent above $680 \mathrm{~nm}$. Thus the spectrum at longer wavelength is due to NV emission and background fluorescence generated within the fiber by the excitation laser, that is not blocked by the mirror coating on the facet. (c) Saturation measurement of the NV emission. (d) Measurement of the intensity correlation $g^{(2)}$ at an excitation power $P=2.5 \mathrm{~mW}$ (excitation power measured after the fiber) revealing the presence of a single NV center. For (c) and (d): The emission has been filtered spectrally in the range $633-647 \mathrm{~nm}$.

We have successfully demonstrated that we are able to excite the NV center through the fiber. In the next step, we replace the microscope objective with a second fiber mirror as output coupling mirror. We call this second fiber out-coupling fiber. We use the same fiber as in the experiments before with one fiber and one plane mirror that have been discussed in the last section (Sect. 5.1). Figure 5.11(a) shows a microscope image of the realized fiber-fiber cavity. 
(a)

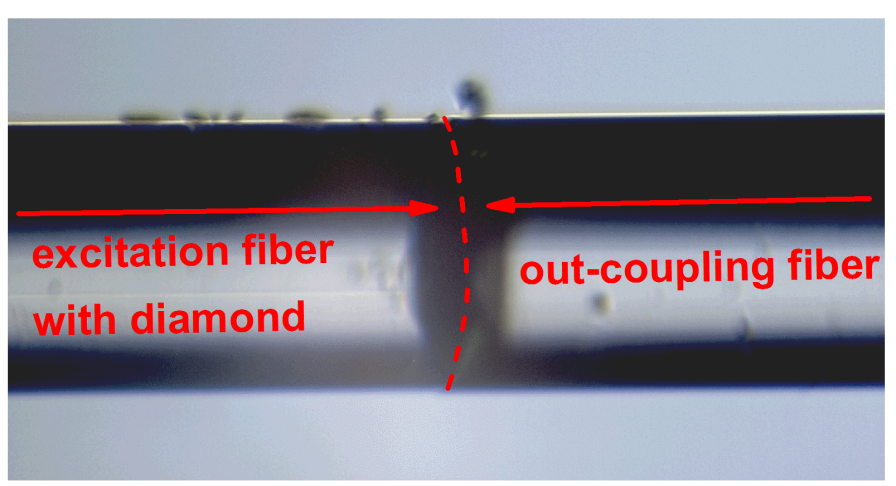

(b)

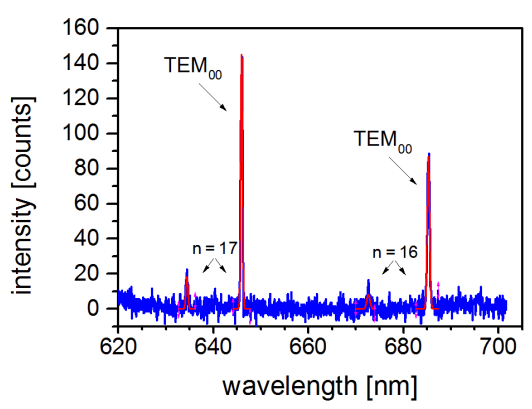

(d)

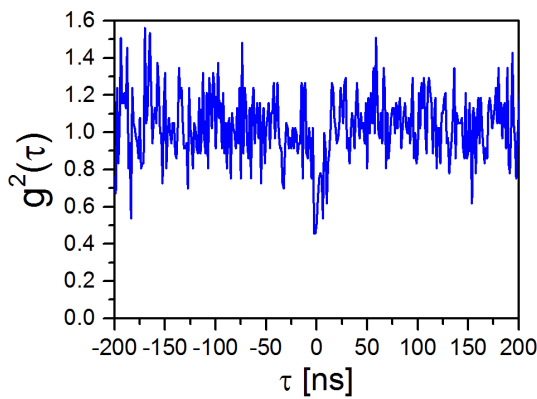

(c)

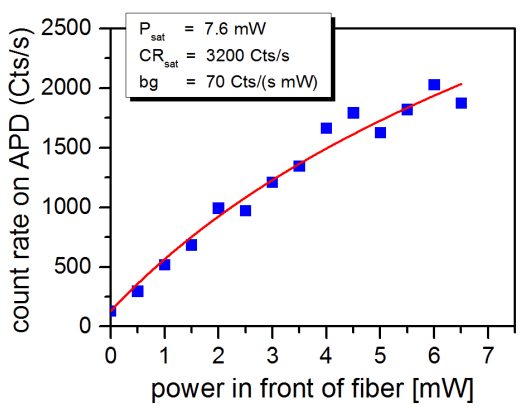

(e)

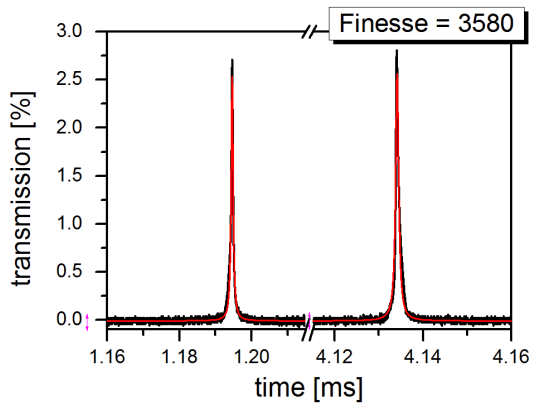

Figure 5.11: (a) Microscope image of the fiber-fiber cavity. There is no more visible gap between the two fibers. (b) Cavity emission spectrum of the NV center coupled to the fiberfiber cavity. The visible modes are fitted with Gaussians. The two fundamental $\mathrm{TEM}_{00}$ modes are at wavelengths $645.9 \mathrm{~nm}$ and 685.3 . Thus the FSR is $\nu_{\mathrm{FSR}}=26.5 \mathrm{THz}$ which corresponds to a cavity length $l=5.6 \mu \mathrm{m}$. (c) Fluorescence count rate as function of excitation power measured in front of the first fiber. Only emission within the wavelength range $633-647 \mathrm{~nm}$ is detected. (d) Measurement of the $g^{(2)}$-function at a excitation power $P=7 \mathrm{~mW}$ (measured in front of the fiber). The same spectral filtering as in (c) has been used. (e) Measurement of the finesse of the cavity: An ECDL at a wavelength $\lambda_{\mathrm{ECDL}}=640 \mathrm{~nm}$ is coupled into the cavity through the first fiber while the cavity length is continuously scanned. 


\begin{tabular}{|c|c|c|c|c|}
\hline $\mathrm{n}$ & mode & $\begin{array}{c}\text { wavelength } \lambda \\
{[\mathrm{nm}]}\end{array}$ & $\begin{array}{c}\text { FMWH } \Delta \lambda \\
{[\mathrm{nm}]}\end{array}$ & $\begin{array}{c}\text { area } A \\
\text { [a.u.] }\end{array}$ \\
\hline \multirow{2}{*}{$\stackrel{\sim}{\sim}$} & $\mathrm{TEM}_{00}$ & 645.9 & 0.40 & 72 \\
\hline & $l+m=2$ & 634.4 & 0.43 & 9.9 \\
\hline \multirow{2}{*}{$\stackrel{0}{-1}$} & $\mathrm{TEM}_{00}$ & 685.3 & 0.52 & 56 \\
\hline & $l+m=2$ & 672.6 & 0.67 & 6.9 \\
\hline
\end{tabular}

Table 5.5: Obtained fit parameters: the center wavelength $\lambda$, the width $\Delta \lambda$ and the area underneath each peak $A$ for the 4 modes visible in the cavity emission spectrum in Fig. 5.11(b). The longitudinal mode number is denoted by $n$.

The emission spectrum of the cavity is displayed in Fig. 5.11(b). The cavity is tuned, such that one of the fundamental $\mathrm{TEM}_{00}$ modes is approximately at the ZPL wavelength. A second fundamental mode and emission into higher transverse modes is also visible. The transverse modes correspond to $l+m=2$ (see Eq. 2.44). The modes corresponding to $l+m=1$ are only visible in case the two fibers are lateral misaligned. The four visible modes are fitted with Gaussians. The obtained fit parameters are summarized in Table 5.5. The FSR is directly deduced from the emission spectrum as $\nu_{\mathrm{FSR}}=26.5 \mathrm{THz}$. This corresponds to a cavity of length $l=5.6 \mu \mathrm{m}$. Thus the two fundamental modes visible in the spectra have the longitudinal mode number $n=17$ and $n=16$. Using the radii of curvature of the two fibers $\left(R_{1}=14.2 \mu \mathrm{m}\right.$ and $R_{2}=$ $71.6 \mu \mathrm{m})$ the mode volume computes to $V_{\text {mod }}=6.1 \mu \mathrm{m}^{3}=23.1 \lambda^{3}$.

We perform a saturation measurement and record the intensity correlation function $g^{(2)}(\tau)$ as shown in Fig. 5.11(c) and (d), respectively. Fitting of the saturation curve yields a saturation count rate of $\mathrm{CR}_{\infty}=3200 \mathrm{Counts} / \mathrm{s}$ and a saturation power of $P_{\text {sat }}=7.6 \mathrm{~mW}$. The power of the excitation laser has been measured before the incoupling lens (Thorlabs, C220TME-A) in front of the first fiber. The background rate has been inferred from $g^{(2)}(\tau=0)$. For both measurements the emission has been spectrally filtered using bandpass filters with a transmission window of $633-647 \mathrm{~nm}$. From the fit of the cavity emission spectrum we deduce that $88 \%$ of the detected emission lies within the fundamental $\mathrm{TEM}_{00}$ mode close to the ZPL. Hence we obtain:

$$
\mathrm{CR}_{\infty}(\lambda=645.9 \mathrm{~nm}, n=17)=2800 \text { Counts } / \mathrm{s}
$$

To determine the finesse of the cavity an ECDL with a wavelength $\lambda=640 \mathrm{~nm}$ is coupled into the cavity and the transmission recorded while the cavity length is continuously scanned. Such a measurement is displayed in Fig. 5.11(e). Averaging over 10 measurements we obtain $\mathcal{F}=3580 \pm 900$, which is the same as measured for an empty cavity without a ND (see Sect. 4.2.4). The total round-trip losses amount to $L=0.05 \%$ (cf. Eq. 4.8).

We again compare our results to the prediction the rate model provides. As the 
emission spectrum of the NV center is not exactly known, we assume the same parameters for the NV center as used in the last section. Table 5.6 lists the input parameters for the rate model and the obtained results, i.e. the diffusion rates $R_{i}$ and the efficiencies $P_{i}$ with which each transition decays into the cavity. The sum of all $P_{i}$ gives the total efficiency, that amounts to $P_{\text {tot }}=1.79 \%$. To determine the effectively detected emission rate we have to take into account the out-coupling ratio through the second fiber mirror $\epsilon_{\text {out }}$ and the spatial overlap between cavity mode and guided fiber mode $\epsilon_{\mathrm{ov}}$. We obtain:

$$
\begin{aligned}
\mathrm{CR}_{\infty, \text { theo }}(\lambda=645.9 \mathrm{~nm}, n=17) & =\mathrm{CR}_{\infty}, \text { into-free-space } \\
& =5700 \text { Counts } / \mathrm{s}
\end{aligned}
$$

As we use the same out-coupling fiber as in the experiments before, we assume the spatial overlap $\epsilon_{\mathrm{ov}}$, which is limited by lateral displacement between fiber core and imprint, to remain unchanged. The full set of parameters of the cavity is listed in table 5.7. The theoretically predicted value (Eq. 5.9) is a factor 2 larger than the actual measured value (Eq. 5.8) but still in good agreement with the experimental results considering the uncertainties of the experimental values for the saturation count rates due to limited excitation power and the unknown exact emission spectra of the NV center, that is here coupled to the fiber-fiber cavity.

\begin{tabular}{|c|c|c|c|c|c|c|c|}
\hline \hline $\mathrm{i}$ & $\begin{array}{c}E_{i} / \hbar \\
{[\mathrm{meV}]}\end{array}$ & $\begin{array}{c}\gamma_{i} \\
{[\mathrm{MHz}]}\end{array}$ & $\begin{array}{c}\gamma^{\star} \\
{[\mathrm{THz}]}\end{array}$ & $\begin{array}{c}\gamma_{i, i-1} \\
{[\mathrm{THz}]}\end{array}$ & $\begin{array}{c}g_{i} \\
{[\mathrm{GHz}]}\end{array}$ & $\begin{array}{c}R_{i} \\
{[\mathrm{KHz}]}\end{array}$ & $\begin{array}{c}P_{i} \\
{[\%]}\end{array}$ \\
\hline 0 & 0 & 0.69 & 15 & - & 1.3 & 20 & 0.057 \\
1 & 31.8 & 2.42 & 15 & 85 & 2.4 & 220 & 0.63 \\
2 & 70.3 & 8.57 & 15 & 82 & 4.6 & 270 & 0.78 \\
3 & 124 & 7.57 & 15 & 79 & 4.3 & 68 & 0.119 \\
4 & 168 & 6.46 & 15 & 88 & 4.0 & 32 & 0.091 \\
5 & 221 & 4.23 & 15 & 65 & 3.2 & 9.0 & 0.026 \\
6 & 275 & 3.03 & 15 & 71 & 2.7 & 4.3 & 0.012 \\
7 & 319 & 1.51 & 15 & 86 & 1.9 & 1.8 & 0.0052 \\
\hline $\mathrm{e}$ & 1941 & - & - & - & - & $P_{\text {tot }}:$ & 1.79 \\
\hline \hline
\end{tabular}

Table 5.6: Full list of parameters for the rate model and the obtained results for the case of a fiber-fiber cavity with effective length $l=5.6 \mu \mathrm{m}$ and resonance frequency at $\lambda_{\text {cavity }}=646 \mathrm{~nm}$ 


\begin{tabular}{|c|c|c|c|c|c|c|c|c|c|c|}
\hline \hline $\begin{array}{c}l \\
{[\mathrm{~nm}]}\end{array}$ & $\begin{array}{c}\lambda \\
{[\mathrm{nm}]}\end{array}$ & $\begin{array}{c}T_{f}(\lambda) \\
{[\%]}\end{array}$ & $\begin{array}{c}T_{p}(\lambda) \\
{[\%]}\end{array}$ & $\begin{array}{c}L \\
{[\%]}\end{array}$ & $\mathcal{F}$ & $\begin{array}{c}\delta \nu \\
{[\mathrm{GHz}]}\end{array}$ & $\begin{array}{c}\kappa \\
{[\mathrm{GHz}]}\end{array}$ & $\begin{array}{c}V_{\text {mod }} \\
{\left[\lambda^{3}\right]}\end{array}$ & $\begin{array}{c}\epsilon_{\text {out }} \\
{[\%]}\end{array}$ & $\begin{array}{c}\epsilon_{\text {ov }} \\
{[\%]}\end{array}$ \\
\hline 5.6 & 645.9 & 0.12 & 0.007 & 0.05 & 3580 & 13 & 47.9 & 22.7 & 68 & 26 \\
\hline \hline
\end{tabular}

Table 5.7: Full list of parameters of the fiber-fiber cavity with length $l=5.6 \mu \mathrm{m}$ and resonance frequency at $\lambda_{\text {cavity }}=645.9 \mathrm{~nm}$. $T_{f}(\lambda)\left(T_{p}(\lambda)\right)$ denote the transmission of the coating on the fiber (plane mirror), $L$ the round-trip losses of the cavity, $\mathcal{F}$ the finesse, $\delta \nu$ the spectral bandwidth, $\boldsymbol{\kappa}$ the decay-rate of the cavity and $V_{\text {mod }}$ the mode volume of the cavity.

With a finesse of $\mathcal{F}=3580$ and mirror separation of $l=5.6 \mu \mathrm{m}$ the cavity linewidth amounts to $\Delta \nu=7.4 \mathrm{GHz}$. Hence, the spectral photon rate of the cavity emission computes to 380 photons $/(\mathrm{s} \mathrm{GHz})$. For the emission into free space we obtain a value of 4.1 photons/(s GHz). Thus cavity coupling increases the spectral photon rate density by two orders of magnitude. This again is a clear proof for cavity enhanced emission beyond simple filtering effects.

We have shown fiber-fiber cavities with lengths down to $l=3.5 \mu \mathrm{m}$. Reducing the mirror separation to this value reduces the mode volume to $V_{\text {mod }}=3.3 \mu \mathrm{m}^{3}$ and increases the efficiency to $P_{\text {tot }}=3.3 \%$, with which the NV center decays into the cavity mode. Thus, nearly a factor of 2 in count rate can easily be gained for the fiber-fiber cavity at room temperature. Even more can be gained using two FIB machined fiber mirrors having both an imprint with small radius on the order of $15 \mu \mathrm{m}$.

In conclusion, we have shown here the successful demonstration of a single NV center coupled two a fiber - fiber cavity, where the obtained results are well reproduced by the rate model. From a practical point these type of cavities are particular attractive: input and output are fiber coupled, a preselected emitter is employed and only one remaining degree-of-freedom, the cavity length, enables fabrication of a simple device with high passive mechanical stability. 


\section{Chapter 6}

\section{Summary and future prospects}

In this work we have for the first time experimentally demonstrated the coupling of a single nitrogen-vacancy (NV) center to different types of fiber-based Fabry-Perot microcavities at room temperature. Furthermore, we also have developed a theoretically framework describing the coupling of a NV center to a microcavity applicable both for room temperature experiments and experiments at cryogenic temperatures.

As motivated in the introduction coupling of a NV center to a cavity is an essential building block in order to implement many quantum information science protocols in a useful practical way. Within this work we have introduced a versatile type of cavity and coupling to a single emitter has been demonstrated. The cavities are based on the realization of a concave mirror on the facet of an optical fiber. Two different fabrication techniques have been successfully applied within this work: in collaboration with the group of J. Reichel at ENS Paris, concave features in the facets with radii of curvature between $R=20-300 \mu \mathrm{m}$ and size diameter $d=12-29 \mu \mathrm{m}$ have been fabricated by laser machining with a $\mathrm{CO}_{2}$ laser. Second, focused ion beam (FIB) milling has been used to produce structures with diameter $d=11 \mu \mathrm{m}$ and radius of curvature $R=14 \mu \mathrm{m}$ into the facets of fibers. Both methods result in very smooth surfaces with surface roughness $\sigma_{\text {rms }}$ of a few Ångström. These machined fiber facets have afterwards been coated with a dielectric mirror stack. With these fibers we here have realized cavities with finesse up to $\mathcal{F}=3500$. Using a different coating consisting of more dielectric layers a higher finesse can in principle be achieved. Finesse values up to $\mathcal{F}=1.5 \times 10^{5}$ have been demonstrated [216].

The fiber-based Fabry-Perot cavities feature many advantages. The resonance frequency can easily be tuned, the cavity-output is automatically fiber-coupled and single emitters in form of NV centers hosted in nanodiamonds (NDs) can directly be incorporated into the cavity. With these cavities a small mode volume can be achieved. We have successfully demonstrated cavities with cavity lengths down to $l=3 \mu \mathrm{m}$. Using a FIB structured fiber, this would result in a mode volume of $V_{\text {mod }}=2.9 \mu \mathrm{m}^{3}=11 \lambda^{3}$ (at $\lambda=640 \mathrm{~nm}$ ). The smallest mode volume we have achieved in this work is $V_{\text {mod }}=3.3 \mu \mathrm{m}^{3}$ using two fiber mirrors separated by $3.5 \mu \mathrm{m}$. 
We have also discussed several technical aspects concerning fiber cavities in order to achieve a successful coupling to a single emitter. To work at the single photon level requires low background fluorescence. If the excitation laser, with a typical power that saturates a NV center, is coupled into a fiber, this generates huge background fluorescence. By using appropriate mirror coatings blocking the excitation laser we were able to circumvent this problem. Furthermore, we have implemented a scheme to stabilize the cavity lengths using two parallel cavities. In first experiments we have applied a side-of-fringe lock and in an improved setup we have employed a PoundDrever-Hall scheme to achieve a higher stability. Using two cavities allows to spatially separate the single photon cavity and the stabilization cavity with the classical light field from the reference laser to which the cavity is stabilized. This prevents background fluorescence within the single photon cavity.

In this work we have used single NV centers hosted in NDs that have been fabricated by a high-pressure-high-temperature (HPHT) process. We have developed a process to deposit well isolated de-agglomerated NDs on a plane mirror: we have used spin coating, where the NDs were dispersed in a suitable solvent, that prevents agglomeration of NDs, evaporates fast and shows a good wetting of the substrate. By measuring the intensity correlation function $g^{(2)}$ we have ensured that the ND investigated further contains only a single NV center. Due to internal strain, which differs from ND to ND, each NV center experiences a slightly different environment resulting in fluctuations of their properties. We focused on two aspects: the emission spectrum and the internal population dynamics. Assuming a Huang-Rhys model we have inferred from fitting the emission spectra the vibronic properties of the NV center. Measuring the intensity correlation function at different excitation powers allowed to gain insights into the internal population dynamics. A simplified effective 3-level model, that takes into account power dependent charge state switching and the meta-stable singlet states of the NV center, allowed to deduce the transition rates between the different levels. We have measured both emission spectra and intensity correlation function for several different NV centers and have discussed the variations in their vibronic properties and internal population dynamics due to different strain each of the NV centers experiences.

Additionally we also have measured the emission spectrum of NV centers hosted in these HPHT NDs at cryogenic temperatures. The linewidth at low temperature is limited by spectral diffusion. We have observed linewidths in the range of few hundred GHz with Gaussian line shape. For many desired future application this linewidth is too large. The main source for spectral diffusion is assumed to be substitutional nitrogen. The route for high quality NDs is to use diamond films fabricated by chemical vapor deposition, where a much higher chemical purity compared to HPHT grown diamonds can be achieved. From these diamond films NDs are produced either via a bead assisted sonic disintegration technique or etching. Investigations towards this goal are on the way in our group.

To theoretically describe the coupling of a single NV center to a microcavity we have expanded a master equation model. To account for the vibronic spectra of the NV center we have assumed a Huang-Rhys model, consisting of one excited state and 
several vibronic ground states. The parameters for this model are deduced from fitting the measured emission spectrum of the NV center. We have taken into account several line broading mechanisms: The dynamic Jahn-Teller effect, which is the dominant linebroading effect for the zero phonon line (ZPL), is accounted for by a pure dephasing rate, which equally affects all transition from the excited state to any of the vibrational ground states. The transitions to higher vibronic ground states, which form the phonon sidebands (PSBs) of the NV center, are broadened by fast relaxation within the vibronic ground states. The coupling of the ZPL and all PSB transitions to the cavity is considered with appropriate coupling rates.

From this master equation picture we have deduced a rate model. This is valid because for most experimental situations non-coherent processes are dominant. The coherences can thus be adiabatically eliminated. The underlying mechanism of the coupling is an off-resonant phonon-assisted cavity feeding from all ZPL and PSB transitions into the cavity mode. At any given wavelength within the NV emission spectrum the ZPL and all PSBs contribute to the cavity emission. Numeric evaluation of the rate model allows to predict the expected photon count rate for a cavity-coupled NV center at room temperature. A few percent of the total NV emission will be channeled into the cavity mode when the cavity resonance is in the range of the full NV emission spectrum. The expected count rate by the rate model is significantly (by several orders of magnitude) larger compared to the case where the cavity acts only as a spectral and spatial filter. In this regime, where the pure dephasing rate $\gamma^{\star}$ and fast ground state relaxation rates $\gamma_{i, i-1}$ are large compared to the cavity decay rate $\boldsymbol{\kappa}$, the efficiency is not limited by the cavity, but only by the emitter and roughly described by a factor of the form $F_{p}^{\star}=\sum_{i} 4 g_{i} /\left[\gamma\left(\gamma^{\star}+\gamma_{i, i-1}\right)\right]$.

When the NV center is cooled to cryogenic temperatures the pure dephasing rate is reduced. The rate model also allowed to investigate this regime. We have shown for the case of the cavity in resonance with the ZPL, the efficiency is dramatically increased and we reach the standard Purcell regime with $F_{p}=4 g^{2} /(\boldsymbol{\kappa} \gamma)$. It is only the contribution from the ZPL which is increased as this transition is not broadened by ground state relaxation. The contributions from the PSBs remain virtually unaltered. Thus, when the cavity resonance is within the PSBs the efficiency only slightly differs from the room temperature case.

Numerical simulation of the full master equation allowed to verify, that the rate model is accurate enough for most experimental conditions. Only for cavities with much higher finesse the full master equation is required to adequately describe the experiments. In case the cavity decay rate and the pure dephasing rate are both smaller than the coherent coupling rate, the onset of strong coupling and oscillation between cavity photon number and excited state population is observed. It is worth mentioning that fiber cavities with finesse necessary to reach this regime are feasible.

In this work we have demonstrated experimentally the coupling of a single NV center to different types of fiber-based Fabry-Perot cavities. In a first experiment we have employed a standard plane mirror and one concave fiber mirror, fabricated with $\mathrm{CO}_{2}$ laser machining with radius of curvature $R=71.6 \mu \mathrm{m}$. Onto the plane mirror 
NDs containing single NV center were deposited. Investigating both, the emission into free space and the cavity-coupled emission for the same single NV center allowed to unambiguously witness cavity-enhanced emission of the NV center and to quantitatively verify our theoretical predictions. We successfully showed tuning of the cavity emission by varying the cavity length $l$ between $6 \mu \mathrm{m}$ and $3 \mu \mathrm{m}$. As an important result we observe single photon emission into the cavity mode over a very broad spectral range, thereby realizing a continuously tunable, narrow band single photon source (SPS). We have observed a saturation count rate of 770 Counts/s when the cavity is in resonance with the ZPL with a spectral bandwidth of $\Delta \nu=46 \mathrm{GHz}$ determined by the cavity linewidth. When the cavity was tuned to the maximum of the PSB a saturation count rate of 3700 Counts/s into a $\Delta \nu=90 \mathrm{GHz}$ broad line was observed. In both cases the resulting spectral photon rate density is higher as it was in the case of free-space emission, where we had measured a saturation count rate of $2.9 \times 10^{5}$ photons in the spectral detection window $650-750 \mathrm{~nm}$ corresponding to $\Delta \nu=62 \mathrm{THz}$. This clearly proves cavity-enhanced emission beyond simple spectral filtering. This is even more evident if one additionally takes into account spatial filtering: the combined filtering effects would yield a saturation count rate of $\approx 2-9$ photons/s, which is $2-3$ orders of magnitude smaller than the actual observed rates. The rate model predicts photon rates for these two experimental condition as $620 \mathrm{Counts} / \mathrm{s}$ for the cavity in resonance with the ZPL and 3700 Counts/s on the maximum of the PSB. Hence we have a very good agreement between theoretical model and experiment. Furthermore, the model also accurately reproduced the intensity of the spectra, that have been recorded while tuning the cavity length from $6 \mu \mathrm{m}$ and $3 \mu \mathrm{m}$, where we observed an increase in photon rate due to the stronger coupling because of the smaller mode volume. The coherent coupling rate on the ZPL was $g_{0}=1.1-1.2 \mathrm{GHz}$ and on the the PSB transitions in the range $g_{i}=1.6-4.4 \mathrm{GHz}$. The pure dephasing rate for this NV center was $\gamma^{\star}=15 \mathrm{THz}$ and the total spontaneous decay rate $\gamma=36.8 \mathrm{MHz}$. The ground state relaxation rates are on the order of $\approx 80 \mathrm{THz}$. The NDs investigated here induced additional losses and reduced the finesse on the ZPL wavelength to approximately $\mathcal{F} \approx 940$. The mode volume for the shortest cavity was $V_{\bmod }=6.5 \mu \mathrm{m}^{3}=24.8 \lambda^{3}$.

The possibility to easily investigate both, emission into free space and cavity-coupled emission is unique for fiber-based Fabry-Perot cavities. They further allow continuously tuning of the coupling strength by varying the mirror separation. This made our system an ideal test bench to study phonon-assisted coupling, a coupling scheme previously unknown for the NV center in diamond.

In a second proof-of-principle experiment we have employed a cavity consisting of two concave fiber mirrors. We have used the same $\mathrm{CO}_{2}$ laser machined out-coupling fiber employed in above described experiments and a FIB fabricated excitation fiber with radius of curvature of $R=14.2 \mu \mathrm{m}$. In collaboration with the group of O. Benson at HU Berlin, a selectively chosen ND containing a single NV center has been transferred with an atomic force microscope tip onto the excitation fiber facet, centered on the fiber core. We have first shown that it is indeed possible to excite the NV center directly via the fiber. However, in contrast to focusing the excitation laser through a microscope 
objective onto the ND, we here needed higher excitation powers to saturate the NV center because of the larger excitation spot determined by the mode field diameter of the excitation fiber. We have measured the cavity coupled emission and obtained a saturation count rate of 2800 counts/s for a cavity tuned close to the ZPL wavelength $\left(\lambda_{\text {cavity }}=646 \mathrm{~nm}\right)$. The finesse was not reduced by the ND and we observed, as for an empty cavity, a value of $\mathcal{F}=3580$. The cavity had a length $l=5.6 \mu \mathrm{m}$ and therefore a mode volume $V_{\text {mod }}=6.1 \mu \mathrm{m}^{3}=22.7 \lambda^{3}$. This results in a coherent coupling rate of the ZPL transition of $g_{0}=1.3 \mathrm{GHz}$. The cavity linewidth amounts to $\Delta \nu=7.4 \mathrm{GHz}$. Hence, the spectral photon rate density is increased from the free-space emission value of 4.1 photons $/(\mathrm{s} \mathrm{GHz})$ to 380 photons $/(\mathrm{s} \mathrm{GHz})$ for the cavity-coupled emission. From a practical point of view this type of cavity is very interesting. Once aligned, the only remaining degree-of-freedom is the cavity length. This enables the fabrication of a simple device with high passive mechanical stability. Furthermore excitation and output are both fiber-coupled. As the NV center is preselected, one can chose its properties.

One of the foci of future experiments is the realization of a SPS: Depending on the application either a SPS with a high efficiency and repetition rate working at ambient conditions or a SPS for indistinguishable photons is desired. For the second case we have identified a favorable working point for the use of the NV center as SPS: Using a cavity that is in resonance with the NV center ZPL with finesse $\mathcal{F}=8 \times 10^{4}$ and mode volume $V_{\text {mod }}=12 \lambda_{\mathrm{ZPL}}^{3}$, the master equation model predicts that $95 \%$ of the total emission is directed into the cavity mode assuming a pure dephasing rate of $\gamma^{\star}=10 \mathrm{GHz}$, which is reached at a temperature of $\approx 30 \mathrm{~K}$. Moreover, due to the much shorter excited state lifetime of the NV center because of the Purcell enhancement, the cavity linewidth is narrower than the lifetime limit. Hence, we have a SPS emitting indistinguishable photons. To have a truly SPS the excitation of the NV center needs also to be considered. The intensity and duration of the excitation pulses need to be tailored in order to have a probability close to unity to excite the NV center and very low chance for multi-photon events. Thus to fully simulate the NV center as SPS pulsed excitation needs also to be included in the master equation. This can be done analogously to the work of $\mathrm{Su}$ et al. [13], where a less sophisticated model of the NV center without pure dephasing was employed. This regime is in reach of experimental realization with a cavity-coupled NV center at cryogenic temperatures. A closed-cycle cryostat with a large sample chamber, into which a fiber cavity will be mounted, is set up right now in our labs and we already have successfully realized a plane mirror - fiber cavity, where the plane mirror was cooled to cryogenic temperatures [240]. To realize a highly efficient SPS at room temperature the NV center is not well suited. However, this can be achieved using a different color center, such as the silicon-vacancy center featuring a short emitter lifetime of $\approx 1$ ns and a large Debye-Waller factor of $\approx 0.8$ with narrow room temperature ZPL linewidth $(\Delta \nu \approx 0.7 \mathrm{~nm})$ [241].

As outlined in the introduction a second field of future investigations is cavity enhanced spin measurements. To manipulate directly the spin state of the NV center a microwave pulse needs to be applied to the NV center. To incorporate the microwave fields with the cavity setup, one might lithographically define microwave transmission 
lines directly on a plane mirror, onto which NDs are spin coated afterwards. In the simplest approach the readout of the spin already profits from an increased collection efficiency. More elaborate schemes make use of a spin-dependent cavity transmission where for instance the reflectivity of the cavity or the phase of a reflected photon is spin-dependent. Because the transition frequency between ground and excited state slightly depends on the electron spin state of the NV center, a cavity tuned to one of these transitions but off-resonant for the others allows discrimination between the spin states. This already works in the weak coupling regime [69] and is doable with the cavities realized in this work. The ultimate goal is to realize a spin-photon interface such that spin and photon are entangled. Interference of two photons from two different cavities containing each a single NV center will lead to entanglement of the two distinct NV centers. This scheme requires indistinguishable photons.

The application of NV centers in magnetometry is based on optical detected magnetic resonances. A higher collection efficiency increases the sensitivity. However, because one is only interested in a large broad band collection efficiency giving rise to high count rates, using photonic structures to improve the collection efficiency, e.g. nanowires as employed by Maletinsky et al. [44], are more promising than using cavities. The nanowires are efficient waveguides for the NV emission and allow a detection efficiency of $>40 \%$ [199]. Using cavities only a few percent can be reached at room temperature. A different approach to detect magnetic fields with the NV center is to detect absorption on the infrared transition between the singlet states. In this case, as proposed by Dumeige et al [242], a cavity can significantly increase the sensitivity.

In summary there are many promising future prospects for cavity-coupled NV centers. Within this work we have established fiber-based cavities which are highly suited for many of these cases. 


\section{Appendix A}

\section{Matlab codes}

Program code calculating the solution (time dependent density matrix) of the master equation as exponential series and calculating the expectation values for spontaneous emission and cavity emission using the quantum toolbox for matlab [189]. (The quantum optics toolbox for matlab is available at http://qo.phy.auckland.ac.nz/ toolbox/):

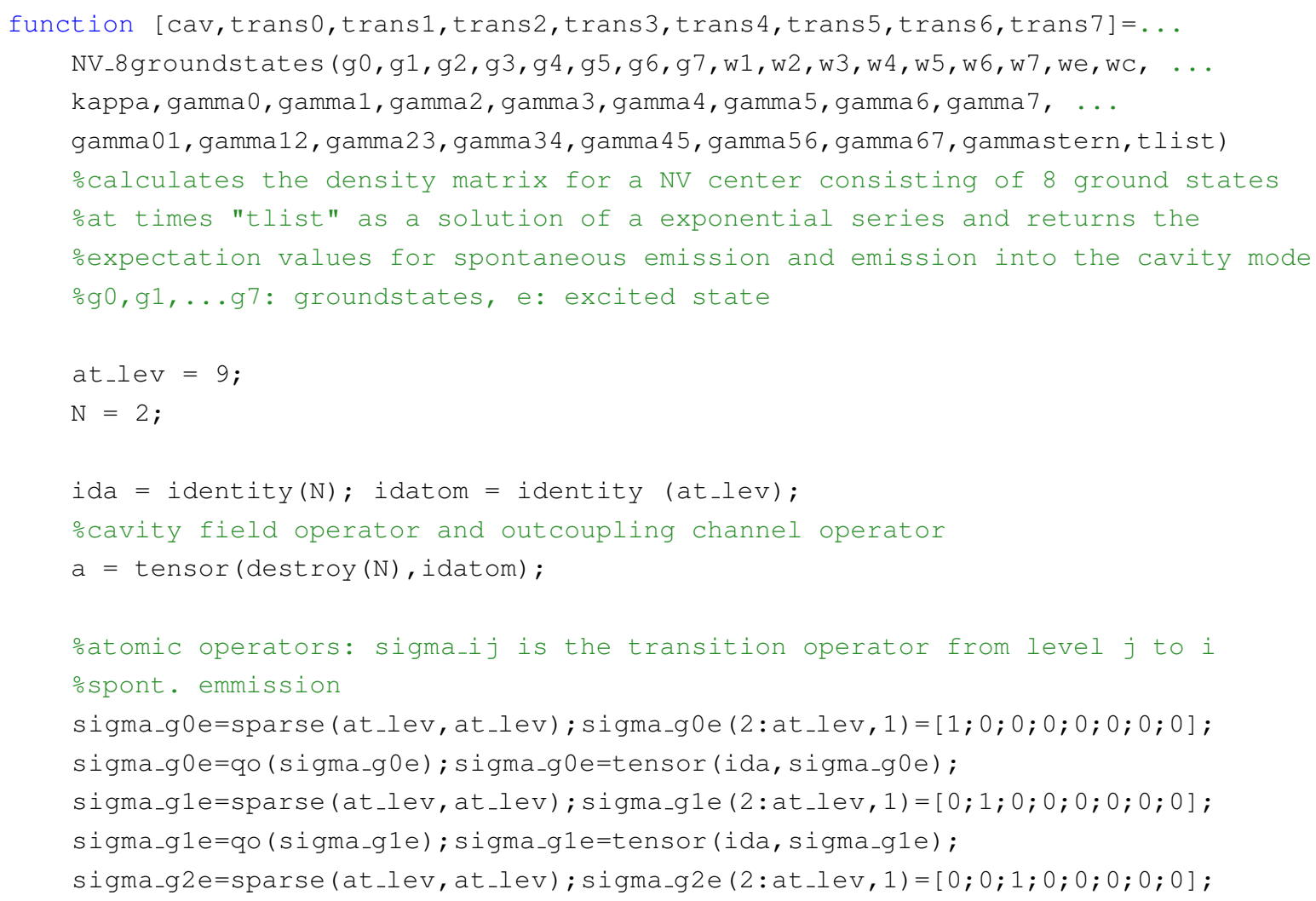


sigma_g2e=qo ( sigma_g2e); sigma_g2e=tensor (ida, sigma_g2e); sigma_g $3 e=s p a r s e\left(a t \_l e v, a t \_l e v\right) ;$ sigma_g $3 e(2$ at_lev, 1$)=[0 ; 0 ; 0 ; 1 ; 0 ; 0 ; 0 ; 0]$; sigma_g3e=qo ( sigma_g3e); sigma_g3e=tensor (ida, sigma_g3e); sigma_g4e=sparse (at_lev, at_lev); sigma_g4e $(2$ :at_lev, 1$)=[0 ; 0 ; 0 ; 0 ; 1 ; 0 ; 0 ; 0]$; sigma_g4e=qo ( sigma_g4e); sigma_g4e=tensor (ida,sigma_g4e); sigma_g5e=sparse (at_lev, at_lev); sigma_g $5 e(2$ :at_lev, 1$)=[0 ; 0 ; 0 ; 0 ; 0 ; 1 ; 0 ; 0]$; sigma_g $5 e=q o($ sigma_g5e); sigma_g5e=tensor (ida,sigma_g5e); sigma_g6e=sparse (at_lev, at_lev); sigma_g6e $(2$ :at_lev, 1$)=[0 ; 0 ; 0 ; 0 ; 0 ; 0 ; 1 ; 0]$; sigma_g6e=qo ( sigma_g6e); sigma_g6e=tensor (ida,sigma_g6e); sigma_g7e=sparse (at_lev, at_lev); sigma_g7e $(2$ : at_lev, 1$)=[0 ; 0 ; 0 ; 0 ; 0 ; 0 ; 0 ; 1]$; sigma_g7e=qo ( sigma_g7e); sigma_g7e=tensor (ida, sigma_g7e);

oground state relaxation

sigma_g0g1=sparse (at_lev, at_lev); sigma_g0g1 (2:at_lev, 3) = $[1 ; 0 ; 0 ; 0 ; 0 ; 0 ; 0 ; 0] ;$ sigma_g0g1= qo (sigma_g0g1); sigma_g0g1=tensor (ida,sigma_g0g1); sigma_glg2=sparse (at_lev, at_lev); sigma_glg2 (2:at_lev,4)= $0 ; 1 ; 0 ; 0 ; 0 ; 0 ; 0 ; 0] ;$ sigma_glg2= qo (sigma_glg2); sigma_glg2=tensor (ida,sigma_g1g2); sigma_g2g3=sparse (at_lev, at_lev); sigma_g2g3 $(2$ :at_lev,5)= $0 ; 0 ; 1 ; 0 ; 0 ; 0 ; 0 ; 0] ;$ sigma_g2g3= qo (sigma_g2g3); sigma_g2g3=tensor (ida,sigma_g2g3); sigma_g3g4=sparse (at_lev, at_lev); sigma_g3g4 (2:at_lev,6)= $0 ; 0 ; 0 ; 1 ; 0 ; 0 ; 0 ; 0] ;$ sigma_g3g4= qo (sigma_g3g4); sigma_g3g4=tensor (ida,sigma_g3g4); sigma_g4g5=sparse (at_lev, at_lev); sigma_g4g5 (2:at_lev, 7$)=[0 ; 0 ; 0 ; 0 ; 1 ; 0 ; 0 ; 0] ;$ sigma_g4g5= qo (sigma_g4g5); sigma_g4g5=tensor (ida,sigma_g4g5); sigma_g5g6=sparse (at_lev, at_lev); sigma_g5g6 (2:at_lev,8)= $0 ; 0 ; 0 ; 0 ; 0 ; 1 ; 0 ; 0]$; sigma_g5g6= qo (sigma_g5g6); sigma_g5g6=tensor (ida,sigma_g5g6); sigma_g6g7=sparse (at_lev, at_lev); sigma_g6g7 (2:at_lev,9)= $0 ; 0 ; 0 ; 0 ; 0 ; 0 ; 1 ; 0] ;$ sigma_g6g7= qo (sigma_g6g7); sigma_g6g7=tensor(ida,sigma_g6g7);

ㅇailtonian

$\mathrm{H}=\mathrm{w} 1 *($ sigma_gle*sigma_gle') $+\mathrm{w} 2 *($ sigma_g2e*sigma_g2e') $+\ldots$ w3* (sigma_g3e*sigma_g3e') +w4*( sigma_g4e*sigma_g4e') + ... w5* (sigma_g5e*sigma_g5e') +w6*( sigma_g6e*sigma_g6e') + ... $w 7 *($ sigma_g7e*sigma_g7e') +we* (sigma_g0e'*sigma_g0e) $+\ldots$ $w C *\left(a^{\prime} \star a\right)+\ldots$ $g 0 *\left(a^{\prime} \star s i g m a \_g 0 e+s i g m a \_g 0 e^{\prime} \star a\right)+g 1 *\left(a ' \star s i g m a \_g 1 e+s i g m a \_g 1 e^{\prime} \star a\right)+\ldots$

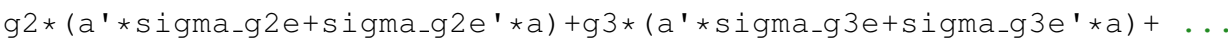

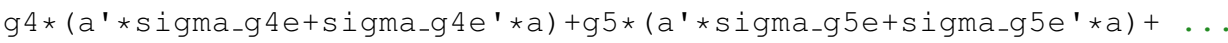
$g 6 *\left(a^{\prime} * s i g m a \_g 6 e+s i g m a \_g 6 e^{\prime} \star a\right)+g 7 *\left(a^{\prime} \star s i g m a \_g 7 e+s i g m a \_g 7 e ' * a\right) ;$

ocollapse operators

$\mathrm{Ca}=\operatorname{sqrt}(\mathrm{kappa}) \star \mathrm{a} ; \mathrm{CadCa}=\mathrm{Ca}{ }^{\prime} \star \mathrm{Ca}$;

$\mathrm{CO}=\operatorname{sqrt}($ gammaO $) \star \mathrm{sigma}_{-} \mathrm{g} 0 \mathrm{e} ; \mathrm{COdCO}=\mathrm{CO}^{\prime} \star \mathrm{CO} ;$

$\mathrm{C} 1=\operatorname{sqrt}\left(\right.$ gamma1) $* \mathrm{sigma}_{-} \mathrm{Ie} ; \mathrm{C} 1 \mathrm{dC} 1=\mathrm{C} 1^{\prime} \star \mathrm{C} 1 ;$

$\mathrm{C} 2=\operatorname{sqrt}($ gamma 2$) \star \mathrm{sigma}_{-} 2 \mathrm{e} ; \mathrm{C} 2 \mathrm{dC} 2=\mathrm{C} 2{ }^{\prime} \star \mathrm{C} 2$; 

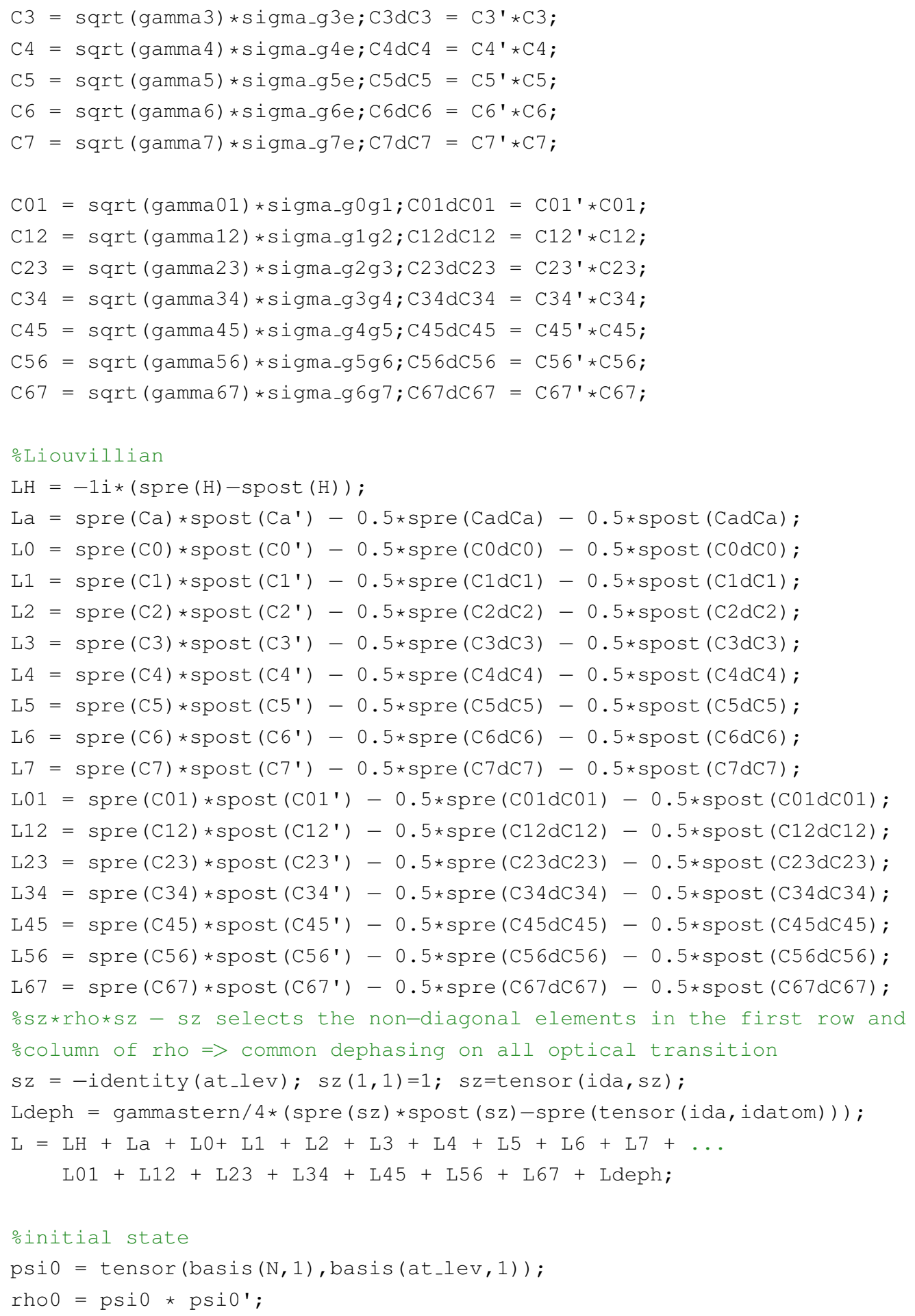


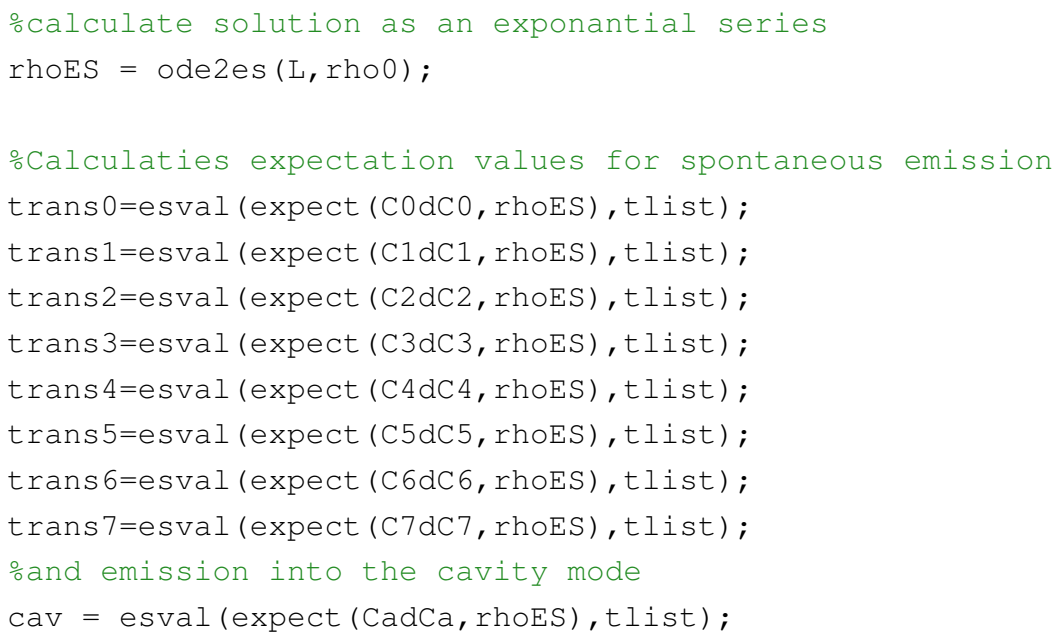

\section{Remark}

To reduce the workload when handling exponential series the quantum optics toolbox employs a simplification routine "estidy.m" which neglects terms with small amplitude and merges terms with similar exponential rates. For the simulation performed in this work the tolerances in this tidying routine have been changed manually to $1 \mathrm{E}-15$ as the input parameters vary over many orders of magnitude. 


\section{Appendix B}

\section{Components}

\section{Microwave-setup}

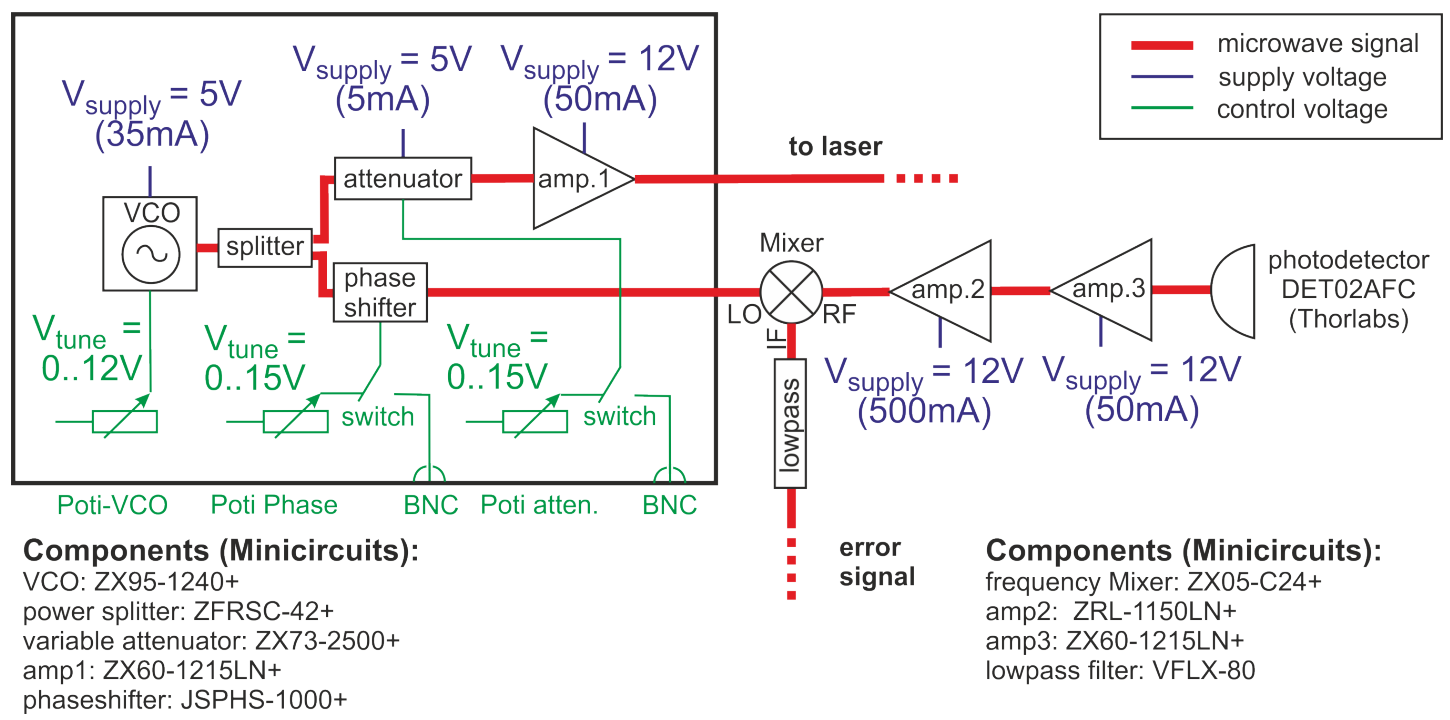

Figure B.1: Microwave components used for stabilization. The signal from a voltage controlled oscillator (VCO) is split and one part coupled to a bias-T of an ECDL (Toptical DL100) to create sidebands at $\approx 1.2 \mathrm{GHz}$. The amplitude is amplified and can be controlled via a voltage controlled variable attenuator. The second line which phase can be controlled is mixed with the amplified signal from a fast photo diode. Lowpass filtering creates the error signal that is fed to the stabilization electronics (Laselock TEM Messtechnik). 


\section{Hanbury-Brown \& Twiss setup}

The HBT setup consist of a non-polarizing nominal 50:50 beam splitter (LensOptics) and two APDs (Picoquant $\tau$-SPAD) with a timing Jitter of $475 \mathrm{ps}$ [243]. The beam splitter has an actual measured splitting ratio of 60:40. In each of the two paths dielectric bandpass filters are mounted. The light is focused with $f=50 \mathrm{~mm}$ lenses onto the active area of the APDS. The active area has a diameter of $150 \mu \mathrm{m}$.

\section{Spectrometer}

All spectra except those in Fig. 3.12 have been measured with the first of the two following spectrometer. Those in Fig. 3.12 with the second.

1. Acton research SpectraPro2500i grating spectrometer with a liquid nitrogen cooled CCD detector (Acton research SP-10 LN/100br). For all spectra a $600 \mathrm{gr} / \mathrm{mm}$ ruled grating with $1 \mu \mathrm{m}$ blaze has been used. Details on the resolution can be found in [243].

2. Horiba Jobin Yvon iHR550 grating spectrometer with a liquid nitrogen cooled CCD detector (Horiba Jobin Yvon Symphony CCD, 1024x256 Pixel, back illuminated, deep depletion). For all spectra a blazed $600 \mathrm{gr} / \mathrm{mm}$ holographic grating has been employed. More details can be found in [244]. 


\section{Appendix C}

\section{Abbreviations}

The following table C.1 lists all abbreviations introduced and used in this work alphabetically:

\begin{tabular}{|c|l|c|l|}
\hline \hline AFM & atomic force microscope & NA & numerical aperture \\
APD & avalanche photo diode & ND & nanodiamond \\
BASD & bead assisted sonic disintegration & NMR & nuclear magnetic resonance \\
CVD & chemical vapor deposition & NV & nitrogen-vacancy \\
cw & continuous wave & ODMR & optical detected magnetic resonance \\
DFT & density functional theory & PC & photonic crystal \\
ECDL & external cavity diode laser & PDH & Pound-Drever-Hall \\
EPR & electron paramatric resonance & PLE & photo luminescence excitation \\
ESR & electron spin resonance & PSB & phonon side band \\
FIB & focused ion beam & PVP & polyvinylpyrrolidone \\
FoM & figure of merit & QD & quantum dot \\
FPI & Fabry-Perot interferometer & QIS & quantum information science \\
FSR & free spectral range & RF & radio frequency \\
FWHM & full width at half maximum & RIE & reactive ion etching \\
GaP & gallium phosphide & SEM & scanning electron microscope \\
GSD & ground state depletion & SiV & silicon-vacancy \\
HBT & Hanbury-Brown \& Twiss & SM & single mode \\
HPHT & high-pressure-high-temperature & SNR & signal to noise ratio \\
IR & infrared & SPS & single photon source \\
MFD & mode field diameter & STED & stimulated emission depletion \\
MM & multi mode & UV & ultraviolet \\
MO & molecular orbital & ZPL & zero phonon line \\
MW & micro wave & \\
\hline \hline
\end{tabular}

Table C.1: list of abbreviations 


\section{Bibliography}

[1] R. Feynman, "Simulating physics with computers," Int. J. Theor. Phys. 21, 467 (1982).

[2] M. A. Nielsen and I. L. Chuang, Quantum Computation and Quantum Information (Cambridge University Press, 2000).

[3] D. P. DiVincenzo, "The Physical Implementation of Quantum Computation," Fortschr. Phys. 48, 771 (2000).

[4] C. D. Clark and C. A. Norris, "Photoluminescence associated with the 1.673, 1.944 and $2.498 \mathrm{eV}$ centres in diamond," J. Phys. C 4, 2223 (1971).

[5] A. Gruber, A. Dräbenstedt, C. Tietz, L. Fleury, J. Wrachtrup, and C. von Borczyskowski, "Scanning Confocal Optical Microscopy and Magnetic Resonance on Single Defect Centers," Science 276, 2012 (1997).

[6] Thomson Reuters, Web of Knowledge: http://apps.webofknowledge.com.

[7] C. Kurtsiefer, S. Mayer, P. Zarda, and H. Weinfurter, "Stable Solid-State Source of Single Photons," Phys. Rev. Lett. 85, 290 (2000).

[8] R. Brouri, A. Beveratos, J. Poizat, and P. Grangier, "Photon antibunching in the fluorescence of individual color centers in diamond," Opt. Lett. 25, 1294 (2000).

[9] A. Beveratos, R. Brouri, T. Gacoin, J. Poizat, and P. Grangier, "Nonclassical radiation from diamond nanocrystals," Phys. Rev. A 64, 061802 (2001).

[10] A. Beveratos, R. Brouri, T. Gacoin, A. Villing, J.-P. Poizat, and P. Grangier, "Single Photon Quantum Cryptography," Phys. Rev. Lett. 89, 187901 (2002).

[11] R. Alléaume, F. Treussart, G. Messin, Y. Dumeige, J. Roch, A. Beveratos, R. Brouri-Tualle, J. Poizat, and P. Grangier, "Experimental open-air quantum key distribution with a single-photon source," New J. Phys. 6, 92 (2004).

[12] J. L. O'Brien, "Optical Quantum Computing," Science 318, 1567 (2007). 
[13] C.-H. Su, A. D. Greentree, and L. C. L. Hollenberg, "Towards a picosecond transform-limited nitrogen-vacancy based single photon source," Opt. Express 16, 6240 (2008).

[14] P. Tamarat, T. Gaebel, J. R. Rabeau, M. Khan, A. D. Greentree, H. Wilson, L. C. L. Hollenberg, S. Prawer, P. Hemmer, F. Jelezko, and J. Wrachtrup, "Stark Shift Control of Single Optical Centers in Diamond," Phys. Rev. Lett. 97, 083002 (2006).

[15] F. Jelezko, T. Gaebel, I. Popa, A. Gruber, and J. Wrachtrup, "Observation of Coherent Oscillations in a Single Electron Spin," Phys. Rev. Lett. 92, 076401 (2004).

[16] G. Balasubramanian, P. Neumann, D. Twitchen, M. Markham, R. Kolesov, N. Mizuochi, J. Isoya, J. Achard, J. Beck, J. Tissler, V. Jacques, P. R. Hemmer, F. Jelezko, and J. Wrachtrup, "Ultralong spin coherence time in isotopically engineered diamond," Nat. Mater. 8, 383 (2009).

[17] K. D. Jahnke, B. Naydenov, T. Teraji, S. Koizumi, T. Umeda, J. Isoya, and F. Jelezko, "Long coherence time of spin qubits in ${ }^{12} \mathrm{C}$ enriched polycrystalline chemical vapor deposition diamond," Appl. Phys. Lett. 101, 012405 (2012).

[18] T. Ishikawa, K.-M. C. Fu, C. Santori, V. M. Acosta, R. G. Beausoleil, H. Watanabe, S. Shikata, and K. M. Itoh, "Optical and Spin Coherence Properties of Nitrogen-Vacancy Centers Placed in a $100 \mathrm{~nm}$ Thick Isotopically Purified Diamond Layer," Nano Lett. 12, 2083 (2012).

[19] G. D. Fuchs, A. L. Falk, V. V. Dobrovitski, and D. D. Awschalom, "Spin Coherence during Optical Excitation of a Single Nitrogen-Vacancy Center in Diamond," Phys. Rev. Lett. 108, 157602 (2012).

[20] G. D. Fuchs, V. V. Dobrovitski, D. M. Toyli, F. J. Heremans, C. D. Weis, T. Schenkel, and D. D. Awschalom, "Excited-state spin coherence of a single nitrogen-vacancy centre in diamond," Nat. Phys. 6, 668 (2010).

[21] F. Shi, X. Rong, N. Xu, Y. Wang, J. Wu, B. Chong, X. Peng, J. Kniepert, R.-S. Schoenfeld, W. Harneit, M. Feng, and J. Du, "Room-Temperature Implementation of the Deutsch-Jozsa Algorithm with a Single Electronic Spin in Diamond," Phys. Rev. Lett. 105, 040504 (2010).

[22] F. Jelezko, T. Gaebel, I. Popa, M. Domhan, A. Gruber, and J. Wrachtrup, "Observation of Coherent Oscillation of a Single Nuclear Spin and Realization of a Two-Qubit Conditional Quantum Gate," Phys. Rev. Lett. 93, 130501 (2004).

[23] T. Gaebel, M. Domhan, C. Wittmann, I. Popa, F. Jelezko, J. Rabeau, A. Greentree, S. Prawer, E. Trajkov, P. Hemmer, and J. Wrachtrup, "Photo- 
chromism in single nitrogen-vacancy defect in diamond," Appl. Phys. B 82, 243 (2006).

[24] R. Hanson, F. M. Mendoza, R. J. Epstein, and D. D. Awschalom, "Polarization and Readout of Coupled Single Spins in Diamond," Phys. Rev. Lett. 97, 087601 (2006).

[25] L. Childress, M. V. G. Dutt, J. M. Taylor, A. S. Zibrov, F. Jelezko, J. Wrachtrup, P. R. Hemmer, and M. D. Lukin, "Coherent Dynamics of Coupled Electron and Nuclear Spin Qubits in Diamond," Science 314, 281 (2006).

[26] M. V. Gurudev Dutt, L. Childress, L. Jiang, E. Togan, J. Maze, F. Jelezko, A. S. Zibrov, P. R. Hemmer, and M. D. Lukin, "Quantum Register Based on Individual Electronic and Nuclear Spin Qubits in Diamond," Science 316, 1312 (2007).

[27] P. Neumann, N. Mizuochi, F. Rempp, P. Hemmer, H. Watanabe, S. Yamasaki, V. Jacques, T. Gaebel, F. Jelezko, and J. Wrachtrup, "Multipartite Entanglement Among Single Spins in Diamond," Science 320, 1326 (2008).

[28] P. Neumann, J. Beck, M. Steiner, F. Rempp, H. Fedder, P. R. Hemmer, J. Wrachtrup, and F. Jelezko, "Single-Shot Readout of a Single Nuclear Spin," Science 329, 542 (2010).

[29] A. Dréau, P. Spinicelli, J. R. Maze, J.-F. Roch, and V. Jacques, "Single-Shot Readout of Multiple Nuclear Spin Qubits in Diamond under Ambient Conditions," Phys. Rev. Lett. 110, 060502 (2013).

[30] L. Jiang, J. S. Hodges, J. R. Maze, P. Maurer, J. M. Taylor, D. G. Cory, P. R. Hemmer, R. L. Walsworth, A. Yacoby, A. S. Zibrov, and M. D. Lukin, "Repetitive Readout of a Single Electronic Spin via Quantum Logic with Nuclear Spin Ancillae," Science 326, 267 (2009).

[31] P. C. Maurer, G. Kucsko, C. Latta, L. Jiang, N. Y. Yao, S. D. Bennett, F. Pastawski, D. Hunger, N. Chisholm, M. Markham, D. J. Twitchen, J. I. Cirac, and M. D. Lukin, "Room-Temperature Quantum Bit Memory Exceeding One Second," Science 336, 1283 (2012).

[32] L. Robledo, L. Childress, H. Bernien, B. Hensen, P. F. A. Alkemade, and R. Hanson, "High-fidelity projective read-out of a solid-state spin quantum register," Nature 477, 574 (2011).

[33] W. Pfaff, T. H. Taminiau, L. Robledo, H. Bernien, M. Markham, D. J. Twitchen, and R. Hanson, "Demonstration of entanglement-by-measurement of solid-state qubits," Nat. Phys. 9, 29 (2013). 
[34] T. van der Sar, Z. H. Wang, M. S. Blok, H. Bernien, T. H. Taminiau, D. M. Toyli, D. A. Lidar, D. D. Awschalom, R. Hanson, and V. V. Dobrovitski, "Decoherenceprotected quantum gates for a hybrid solid-state spin register," Nature 484, 82 (2012).

[35] X. Xu, Z. Wang, C. Duan, P. Huang, P. Wang, Y. Wang, N. Xu, X. Kong, F. Shi, X. Rong, and J. Du, "Coherence-Protected Quantum Gate by Continuous Dynamical Decoupling in Diamond," Phys. Rev. Lett. 109, 070502 (2012).

[36] F. Dolde, I. Jakobi, B. Naydenov, N. Zhao, S. Pezzagna, C. Trautmann, J. Meijer, P. Neumann, F. Jelezko, and J. Wrachtrup, "Room-temperature entanglement between single defect spins in diamond," Nat. Phys. 9, 139 (2013).

[37] E. Rittweger, K. Y. Han, S. E. Irvine, C. Eggeling, and S. W. Hell, "STED microscopy reveals crystal colour centres with nanometric resolution," Nat. Photonics 3, 144 (2009).

[38] K. Y. Han, S. K. Kim, C. Eggeling, and S. W. Hell, "Metastable Dark States Enable Ground State Depletion Microscopy of Nitrogen Vacancy Centers in Diamond with Diffraction-Unlimited Resolution," Nano Lett. 10, 3199 (2010).

[39] G. Balasubramanian, I. Y. Chan, R. Kolesov, M. Al-Hmoud, J. Tisler, C. Shin, C. Kim, A. Wojcik, P. R. Hemmer, A. Krueger, T. Hanke, A. Leitenstorfer, R. Bratschitsch, F. Jelezko, and J. Wrachtrup, "Nanoscale imaging magnetometry with diamond spins under ambient conditions," Nature 455, 648 (2008).

[40] J. M. Taylor, P. Cappellaro, L. Childress, L. Jiang, D. Budker, P. R. Hemmer, A. Yacoby, R. Walsworth, and M. D. Lukin, "High-sensitivity diamond magnetometer with nanoscale resolution," Nat. Phys. 4, 810 (2008).

[41] J. R. Maze, P. L. Stanwix, J. S. Hodges, S. Hong, J. M. Taylor, P. Cappellaro, L. Jiang, M. V. G. Dutt, E. Togan, A. S. Zibrov, A. Yacoby, R. L. Walsworth, and M. D. Lukin, "Nanoscale magnetic sensing with an individual electronic spin in diamond," Nature 455, 644 (2008).

[42] B. M. Chernobrod and G. P. Berman, "Spin microscope based on optically detected magnetic resonance," J. Appl. Phys. 97, 014903 (2005).

[43] J. Tisler, T. Oeckinghaus, R. J. Stöhr, R. Kolesov, R. Reuter, F. Reinhard, and J. Wrachtrup, "Single Defect Center Scanning Near-Field Optical Microscopy on Graphene," Nano Lett. 13, 3152 (2013).

[44] P. Maletinsky, S. Hong, M. S. Grinolds, B. Hausmann, M. D. Lukin, R. L. Walsworth, M. Loncar, and A. Yacoby, "A robust scanning diamond sensor for nanoscale imaging with single nitrogen-vacancy centres," Nat. Nanotechnol. 7, 320 (2012). 
[45] L. P. McGuinness, Y. Yan, A. Stacey, D. A. Simpson, L. T. Hall, D. Maclaurin, S. Prawer, P. Mulvaney, J. Wrachtrup, F. Caruso, R. E. Scholten, and L. C. L. Hollenberg, "Quantum measurement and orientation tracking of fluorescent nanodiamonds inside living cells," Nat. Nanotechnol. 6, 358 (2011).

[46] F. C. Ziem, N. S. Götz, A. Zappe, S. Steinert, and J. Wrachtrup, "Highly Sensitive Detection of Physiological Spins in a Microfluidic Device," Nano Lett. 13, 4093 (2013).

[47] F. Dolde, H. Fedder, M. W. Doherty, T. Noebauer, F. Rempp, G. Balasubramanian, T. Wolf, F. Reinhard, L. C. L. Hollenberg, F. Jelezko, and J. Wrachtrup, "Electric-field sensing using single diamond spins," Nat. Phys. 7, 459 (2011).

[48] V. M. Acosta, E. Bauch, M. P. Ledbetter, A. Waxman, L. . S. Bouchard, and D. Budker, "Temperature Dependence of the Nitrogen-Vacancy Magnetic Resonance in Diamond," Phys. Rev. Lett. 104, 070801 (2010).

[49] P. Neumann, I. Jakobi, F. Dolde, C. Burk, R. Reuter, G. Waldherr, J. Honert, T. Wolf, A. Brunner, J. H. Shim, D. Suter, H. Sumiya, J. Isoya, and J. Wrachtrup, "High-Precision Nanoscale Temperature Sensing Using Single Defects in Diamond," Nano Lett. 13, 2738 (2013).

[50] D. M. Toyli, D. J. Christle, A. Alkauskas, B. B. Buckley, C. G. Van de Walle, and D. D. Awschalom, "Measurement and Control of Single Nitrogen-Vacancy Center Spins above 600 K," Phys. Rev. X 2, 031001 (2012).

[51] N. Yao, L. Jiang, A. Gorshkov, P. Maurer, G. Giedke, J. Cirac, and M. Lukin, "Scalable architecture for a room temperature solid-state quantum information processor," Nat. Commun. 3, 800 (2012).

[52] J. Meijer, B. Burchard, M. Domhan, C. Wittmann, T. Gaebel, I. Popa, F. Jelezko, and J. Wrachtrup, "Generation of single color centers by focused nitrogen implantation," Appl. Phys. Lett. 87, 261909 (2005).

[53] J. Meijer, S. Pezzagna, T. Vogel, B. Burchard, H. Bukow, I. Rangelow, Y. Sarov, H. Wiggers, I. Plümel, F. Jelezko, J. Wrachtrup, F. Schmidt-Kaler, W. Schnitzler, and K. Singer, "Towards the implanting of ions and positioning of nanoparticles with nm spatial resolution," Appl. Phys. A 91, 567 (2008).

[54] D. M. Toyli, C. D. Weis, G. D. Fuchs, T. Schenkel, and D. D. Awschalom, "ChipScale Nanofabrication of Single Spins and Spin Arrays in Diamond," Nano Lett. 10, 3168 (2010).

[55] S. Pezzagna, B. Naydenov, F. Jelezko, J. Wrachtrup, and J. Meijer, "Creation efficiency of nitrogen-vacancy centres in diamond," New J. Phys. 12, 065017 (2010). 
[56] S. Pezzagna, D. Rogalla, H.-W. Becker, I. Jakobi, F. Dolde, B. Naydenov, J. Wrachtrup, F. Jelezko, C. Trautmann, and J. Meijer, "Creation of colour centres in diamond by collimated ion-implantation through nano-channels in mica," Phys. Status Solidi A 208, 2017 (2011).

[57] P. Spinicelli, A. Dreau, L. Rondin, F. Silva, J. Achard, S. Xavier, S. Bansropun, T. Debuisschert, S. Pezzagna, J. Meijer, V. Jacques, and J.-F. Roch, "Engineered arrays of nitrogen-vacancy color centers in diamond based on implantation of $\mathrm{CN}^{-}$molecules through nanoapertures," New J. Phys. 13, 025014 (2011).

[58] Y. Kubo, F. R. Ong, P. Bertet, D. Vion, V. Jacques, D. Zheng, A. Dréau, J.F. Roch, A. Auffeves, F. Jelezko, J. Wrachtrup, M. F. Barthe, P. Bergonzo, and D. Esteve, "Strong Coupling of a Spin Ensemble to a Superconducting Resonator," Phys. Rev. Lett. 105, 140502 (2010).

[59] Y. Kubo, C. Grezes, A. Dewes, T. Umeda, J. Isoya, H. Sumiya, N. Morishita, H. Abe, S. Onoda, T. Ohshima, V. Jacques, A. Dréau, J.-F. Roch, I. Diniz, A. Auffeves, D. Vion, D. Esteve, and P. Bertet, "Hybrid Quantum Circuit with a Superconducting Qubit Coupled to a Spin Ensemble," Phys. Rev. Lett. 107, 220501 (2011).

[60] O. Arcizet, V. Jacques, A. Siria, P. Poncharal, P. Vincent, and S. Seidelin, "A single nitrogen-vacancy defect coupled to a nanomechanical oscillator," Nat. Phys. 7, 879 (2011).

[61] P. Rabl, S. J. Kolkowitz, F. H. L. Koppens, J. G. E. Harris, P. Zoller, and M. D. Lukin, "A quantum spin transducer based on nanoelectromechanical resonator arrays," Nat. Phys. 6, 602 (2010).

[62] S. D. Barrett and P. Kok, "Efficient high-fidelity quantum computation using matter qubits and linear optics," Phys. Rev. A 71, 060310 (2005).

[63] E. Togan, Y. Chu, A. S. Trifonov, L. Jiang, J. Maze, L. Childress, M. V. G. Dutt, A. S. Sorensen, P. R. Hemmer, A. S. Zibrov, and M. D. Lukin, "Quantum entanglement between an optical photon and a solid-state spin qubit," Nature 466, 730 (2010).

[64] A. Sipahigil, M. L. Goldman, E. Togan, Y. Chu, M. Markham, D. J. Twitchen, A. S. Zibrov, A. Kubanek, and M. D. Lukin, "Quantum Interference of Single Photons from Remote Nitrogen-Vacancy Centers in Diamond," Phys. Rev. Lett. 108, 143601 (2012).

[65] H. Bernien, L. Childress, L. Robledo, M. Markham, D. Twitchen, and R. Hanson, "Two-Photon Quantum Interference from Separate Nitrogen Vacancy Centers in Diamond," Phys. Rev. Lett. 108, 043604 (2012). 
[66] A. B. Young, C. Y. Hu, and J. G. Rarity, "Generating entanglement with low-Qfactor microcavities," Phys. Rev. A 87, 012332 (2013).

[67] E. Purcell, "Spontaneous Emission Probabilities at Radio Frequencies," Phys. Rev. 69, 681 (1946).

[68] V. M. Acosta, E. Bauch, A. Jarmola, L. J. Zipp, M. P. Ledbetter, and D. Budker, "Broadband magnetometry by infrared-absorption detection of nitrogen-vacancy ensembles in diamond," Appl. Phys. Lett. 97, 174104 (2010).

[69] A. Young, C. Y. Hu, L. Marseglia, J. P. Harrison, J. L. O’Brien, and J. G. Rarity, "Cavity enhanced spin measurement of the ground state spin of an NV center in diamond," New J. Phys. 11, 013007 (2009).

[70] J. I. Cirac, P. Zoller, H. J. Kimble, and H. Mabuchi, "Quantum State Transfer and Entanglement Distribution among Distant Nodes in a Quantum Network," Phys. Rev. Lett. 78, 3221 (1997).

[71] K. Vahala, "Optical Microcavities," Nature 424, 839 (2003).

[72] S. Schietinger, T. Schröder, and O. Benson, "One-by-One Coupling of Single Defect Centers in Nanodiamonds to High-Q Modes of an Optical Microresonator," Nano Lett. 8, 3911 (2008).

[73] S. Schietinger and O. Benson, "Coupling single NV-centres to high- Q whispering gallery modes of a preselected frequency-matched microresonator," J. Phys. B 42, 114001 (2009).

[74] M. Larsson, K. N. Dinyari, and H. Wang, "Composite Optical Microcavity of Diamond Nanopillar and Silica Microsphere," Nano Lett. 9, 1447 (2009).

[75] P. E. Barclay, C. Santori, K.-M. Fu, R. G. Beausoleil, and O. Painter, "Coherent interference effects in a nano-assembled diamond NV center cavity-QED system," Opt. Express 17, 8081 (2009).

[76] M. Gregor, R. Henze, T. Schröder, and O. Benson, "On-demand positioning of a preselected quantum emitter on a fiber-coupled toroidal microresonator," Appl. Phys. Lett. 95, 153110 (2009).

[77] P. E. Barclay, K.-M. C. Fu, C. Santori, and R. G. Beausoleil, "Chip-based microcavities coupled to nitrogen-vacancy centers in single crystal diamond," Appl. Phys. Lett. 95, 191115 (2009).

[78] D. Englund, B. Shields, K. Rivoire, F. Hatami, J. Vuckovic, H. Park, and M. D. Lukin, "Deterministic Coupling of a Single Nitrogen Vacancy Center to a Photonic Crystal Cavity," Nano Lett. 10, 3922 (2010). 
[79] A. W. Schell, G. Kewes, T. Schröder, J. Wolters, T. Aichele, and O. Benson, "A scanning probe-based pick-and-place procedure for assembly of integrated quantum optical hybrid devices," Rev. Sci. Instrum. 82, 073709 (2011).

[80] J. Wolters, A. W. Schell, G. Kewes, N. Nüsse, M. Schoengen, H. Döscher, T. Hannappel, B. Löchel, M. Barth, and O. Benson, "Enhancement of the zero phonon line emission from a single nitrogen vacancy center in a nanodiamond via coupling to a photonic crystal cavity," Appl. Phys. Lett. 97, 141108 (2010).

[81] T. van der Sar, E. C. Heeres, G. M. Dmochowski, G. de Lange, L. Robledo, T. H. Oosterkamp, and R. Hanson, "Nanopositioning of a diamond nanocrystal containing a single nitrogen-vacancy defect center," Appl. Phys. Lett. 94, 173104 (2009).

[82] T. van der Sar, J. Hagemeier, W. Pfaff, E. C. Heeres, S. M. Thon, H. Kim, P. M. Petroff, T. H. Oosterkamp, D. Bouwmeester, and R. Hanson, "Deterministic nanoassembly of a coupled quantum emitter-photonic crystal cavity system," Appl. Phys. Lett. 98, 193103 (2011).

[83] Y. Dumeige, R. Alléaume, P. Grangier, F. Treussart, and J.-F. Roch, "Controling the single-diamond nitrogen-vacancy color center photoluminescence spectrum with a Fabry-Perot microcavity," New J. Phys. 13, 025015 (2011).

[84] Y.-S. Park, A. K. Cook, and H. Wang, "Cavity QED with Diamond Nanocrystals and Silica Microspheres," Nano Lett. 6, 2075 (2006).

[85] K.-M. C. Fu, P. E. Barclay, C. Santori, A. Faraon, and R. G. Beausoleil, "Lowtemperature tapered-fiber probing of diamond nitrogen-vacancy ensembles coupled to GaP microcavities," New J. Phys. 13, 055023 (2011).

[86] P. E. Barclay, K.-M. C. Fu, C. Santori, A. Faraon, and R. G. Beausoleil, "Hybrid Nanocavity Resonant Enhancement of Color Center Emission in Diamond," Phys. Rev. X 1, 011007 (2011).

[87] C. F. Wang, Y.-S. Choi, J. C. Lee, E. L. Hu, J. Yang, and J. E. Butler, "Observation of whispering gallery modes in nanocrystalline diamond microdisks," Appl. Phys. Lett. 90, 081110 (2007).

[88] C. F. Wang, R. Hanson, D. D. Awschalom, E. L. Hu, T. Feygelson, J. Yang, and J. E. Butler, "Fabrication and characterization of two-dimensional photonic crystal microcavities in nanocrystalline diamond," Appl. Phys. Lett. 91, 201112 (2007).

[89] T. M. Babinec, J. T. Choy, K. J. M. Smith, M. Khan, and M. Lončar, "Design and focused ion beam fabrication of single crystal diamond nanobeam cavities," J. Vac. Sci. Technol. B 29, 010601 (2011). 
[90] J. Riedrich-Möller, L. Kipfstuhl, C. Hepp, E. Neu, C. Pauly, F. Mücklich, A. Baur, M. Wandt, S. Wolff, M. Fischer, S. Gsell, M. Schreck, and C. Becher, "One- and two-dimensional photonic crystal microcavities in single crystal diamond," Nat. Nanotechnol. 7, 69 (2012).

[91] J. P. Goss, R. Jones, S. J. Breuer, P. R. Briddon, and S. Öberg, "The Twelve-Line $1.682 \mathrm{eV}$ Luminescence Center in Diamond and the Vacancy-Silicon Complex," Phys. Rev. Lett. 77, 3041 (1996).

[92] A. Faraon, P. E. Barclay, C. Santori, K.-M. C. Fu, and R. G. Beausoleil, "Resonant enhancement of the zero-phonon emission from a colour centre in a diamond cavity," Nat. Photonics 5, 301 (2011).

[93] A. Faraon, C. Santori, Z. Huang, V. M. Acosta, and R. G. Beausoleil, "Coupling of Nitrogen-Vacancy Centers to Photonic Crystal Cavities in Monocrystalline Diamond," Phys. Rev. Lett. 109, 033604 (2012).

[94] B. J. M. Hausmann, B. Shields, Q. Quan, P. Maletinsky, M. McCutcheon, J. T. Choy, T. M. Babinec, A. Kubanek, A. Yacoby, M. D. Lukin, and M. Lončar, "Integrated Diamond Networks for Quantum Nanophotonics," Nano Lett. 12, 1578 (2012).

[95] A. Faraon, C. Santori, Z. Huang, K.-M. C. Fu, V. M. Acosta, D. Fattal, and R. G. Beausoleil, "Quantum photonic devices in single-crystal diamond," New J. Phys. 15, 025010 (2013).

[96] M. W. Doherty, N. B. Manson, P. Delaney, F. Jelezko, J. Wrachtrup, and L. C. Hollenberg, "The nitrogen-vacancy colour centre in diamond," Phys. Rep. 528, 1 (2013).

[97] G. Davies, G and M. Hamer, "Optical studies of $1.945 \mathrm{eV}$ vibronic band in diamond," Proc. R. Soc. Lon. Ser. A 348, 285 (1976).

[98] G. Davies, S. C. Lawson, A. T. Collins, A. Mainwood, and S. J. Sharp, "Vacancyrelated centers in diamond," Phys. Rev. B 46, 13157 (1992).

[99] J. H. N. Loubser and J. A. van Wyk, "Electron spin resonance in the study of diamond," Rep. Prog. Phys. 41, 1201 (1978).

[100] Y. Mita, "Change of absorption spectra in type-Ib diamond with heavy neutron irradiation," Phys. Rev. B 53, 11360 (1996).

[101] G. Davies, "Dynamic Jahn-Teller distortions at trigonal optical centres in diamond," J. Phys. C 12, 2551 (1979).

[102] A. T. Collins, M. F. Thomaz, and M. I. B. Jorge, "Luminescence decay time of the 1.945 eV centre in type Ib diamond," J. Phys. C 16, 2177 (1983). 
[103] L. J. Rogers, S. Armstrong, M. J. Sellars, and N. B. Manson, "Infrared emission of the NV centre in diamond: Zeeman and uniaxial stress studies," New J. Phys. 10, 103024 (2008).

[104] N. B. Manson, J. P. Harrison, and M. J. Sellars, "Nitrogen-vacancy center in diamond: Model of the electronic structure and associated dynamics," Phys. Rev. B 74, 104303 (2006).

[105] V. M. Acosta, A. Jarmola, E. Bauch, and D. Budker, "Optical properties of the nitrogen-vacancy singlet levels in diamond," Phys. Rev. B 82, 201202R (2010).

[106] L. Robledo, H. Bernien, T. van der Sar, and R. Hanson, "Spin dynamics in the optical cycle of single nitrogen-vacancy centres in diamond," New J. Phys. 13, 025013 (2011).

[107] E. van Oort, N. B. Manson, and M. Glasbeek, "Optically detected spin coherence of the diamond N-V centre in its triplet ground state," J. Phys. C 21, 4385 (1988).

[108] J. Harrison, M. Sellars, and N. Manson, "Optical spin polarisation of the N-V centre in diamond," J. Lumin. 107, 245 (2004).

[109] N. Reddy, N. Manson, and E. Krausz, "Two-laser spectral hole burning in a colour centre in diamond," J. Lumin. 38, 46 (1987).

[110] K. Holliday, N. B. Manson, M. Glasbeek, and E. van Oort, "Optical hole-bleaching by level anti-crossing and cross relaxation in the N-V centre in diamond," J. Phys. 1, 7093 (1989).

[111] D. A. Redman, S. Brown, R. H. Sands, and S. C. Rand, "Spin Dynamics and Electronic States of N-V Centers in Diamond by EPR and Four-Wave-Mixing Spectroscopy," Phys. Rev. Lett. 67, 3420 (1991).

[112] N. Manson, X. He, and P. Fisk, "Raman heterodyne detected electron-nucleardouble-resonance measurements of the nitrogen-vacancy center in diamond," Opt. Lett. 15, 1094 (1990).

[113] X. He, X., N. Manson, and P. Fisk, "Paramagnetic resonance of photoexcited $\mathrm{N}-\mathrm{V}$ defects in diamond. I. Level anticrossing in the 3A ground state," Phys. Rev. B 47, 8809 (1993).

[114] A. Lenef and S. Rand, "Electronic structure of the N-V center in diamond: Theory," Phys. Rev. B 53, 13441 (1996).

[115] J. P. Goss, R. Jones, P. R. Briddon, G. Davies, A. T. Collins, A. Mainwood, J. A. van Wyk, J. M. Baker, M. E. Newton, A. M. Stoneham, and S. C. Lawson, "Comment on "Electronic structure of the N-V center in diamond: Theory"," Phys. Rev. B 56, 16031 (1997). 
[116] N. B. Manson and R. L. McMurtrie, "Issues concerning the nitrogen-vacancy center in diamond," J. Lumin. 127, 98 (2007).

[117] M. W. Doherty, N. B. Manson, P. Delaney, and L. C. L. Hollenberg, "The negatively charged nitrogen-vacancy centre in diamond: the electronic solution," New J. Phys. 13, 025019 (2011).

[118] J. R. Maze, A. Gali, E. Togan, Y. Chu, A. Trifonov, E. Kaxiras, and M. D. Lukin, "Properties of nitrogen-vacancy centers in diamond: the group theoretic approach," New J. Phys. 13, 025025 (2011).

[119] M. W. Doherty, F. Dolde, H. Fedder, F. Jelezko, J. Wrachtrup, N. B. Manson, and L. C. L. Hollenberg, "Theory of the ground-state spin of the $\mathrm{NV}^{-}$center in diamond," Phys. Rev. B 85, 205203 (2012).

[120] P. Tamarat, N. B. Manson, J. P. Harrison, R. L. McMurtrie, A. Nizovtsev, C. Santori, R. G. Beausoleil, P. Neumann, T. Gaebel, F. Jelezko, P. Hemmer, and J. Wrachtrup, "Spin-flip and spin-conserving optical transitions of the nitrogenvacancy centre in diamond," New J. Phys. 10, 045004 (2008).

[121] A. Batalov, V. Jacques, F. Kaiser, P. Siyushev, P. Neumann, L. J. Rogers, R. L. McMurtrie, N. B. Manson, F. Jelezko, and J. Wrachtrup, "Low Temperature Studies of the Excited-State Structure of Negatively Charged Nitrogen-Vacancy Color Centers in Diamond," Phys. Rev. Lett. 102, 195506 (2009).

[122] G. D. Fuchs, V. V. Dobrovitski, R. Hanson, A. Batra, C. D. Weis, T. Schenkel, and D. D. Awschalom, "Excited-State Spectroscopy Using Single Spin Manipulation in Diamond," Phys. Rev. Lett. 101, 117601 (2008).

[123] P. Neumann, R. Kolesov, V. Jacques, J. Beck, J. Tisler, A. Batalov, L. Rogers, N. B. Manson, G. Balasubramanian, F. Jelezko, and J. Wrachtrup, "Excited-state spectroscopy of single NV defects in diamond using optically detected magnetic resonance," New J. Phys. 11, 013017 (2009).

[124] L. J. Rogers, R. L. McMurtrie, M. J. Sellars, and N. B. Manson, "Time-averaging within the excited state of the nitrogen-vacancy centre in diamond," New J. Phys. 11, 063007 (2009).

[125] P. Delaney, J. C. Greer, and J. A. Larsson, "Spin-Polarization Mechanisms of the Nitrogen-Vacancy Center in Diamond," Nano Lett. 10, 610 (2010).

[126] Y. Ma, M. Rohlfing, and A. Gali, "Excited states of the negatively charged nitrogen-vacancy color center in diamond," Phys. Rev. B 81, 041204 (2010).

[127] N. Manson, L. Rogers, M. Doherty, and L. Hollenberg, "Optically induced spin polarisation of the $\mathrm{NV}^{-}$centre in diamond: role of electron-vibration interaction," arXiv:1011.2840 (2010). 
[128] A. Batalov, C. Zierl, T. Gaebel, P. Neumann, I. Y. Chan, G. Balasubramanian, P. R. Hemmer, F. Jelezko, and J. Wrachtrup, "Temporal coherence of photons emitted by single nitrogen-vacancy defect centers in diamond using optical Rabioscillations," Phys. Rev. Lett. 100, 077401 (2008).

[129] A. Stoneham, Theory of Defects in Solids (Oxford University Press Inc, New York, 1975).

[130] G. Davies, "The Jahn-Teller effect and vibronic coupling at deep levels in diamond," Rep. Prog. Phys. 44, 787 (1981).

[131] G. Davies, "Vibronic spectra in diamond," J. Phys. C 7, 3797 (1974).

[132] A. Gali, T. Simon, and J. E. Lowther, "An ab initio study of local vibration modes of the nitrogen-vacancy center in diamond," New J. Phys. 13, 025016 (2011).

[133] T. A. Abtew, Y. Y. Sun, B.-C. Shih, P. Dev, S. B. Zhang, and P. Zhang, "Dynamic Jahn-Teller Effect in the NV ${ }^{-}$Center in Diamond," Phys. Rev. Lett. 107, 146403 (2011).

[134] J. Zhang, C.-Z. Wang, Z. Z. Zhu, and V. V. Dobrovitski, "Vibrational modes and lattice distortion of a nitrogen-vacancy center in diamond from first-principles calculations," Phys. Rev. B 84, 035211 (2011).

[135] V. M. Huxter, T. A. A. Oliver, D. Budker, and G. R. Fleming, "Vibrational and electronic dynamics of nitrogen-vacancy centres in diamond revealed by twodimensional ultrafast spectroscopy," Nat. Phys. 9, 744 (2013).

[136] K.-M. C. Fu, C. Santori, P. E. Barclay, L. J. Rogers, N. B. Manson, and R. G. Beausoleil, "Observation of the Dynamic Jahn-Teller Effect in the Excited States of Nitrogen-Vacancy Centers in Diamond," Phys. Rev. Lett. 103, 256404 (2009).

[137] V. Hizhnyakov, V. Boltrushko, H. Kaasik, and I. Sildos, "Strong Jahn-Teller effect in the excited state: Anomalous temperature dependence of the zero-phonon line,"

J. Chem. Phys. 119, 6290 (2003).

[138] A. Maradudin, "Theoretical and Experimental Aspects of the Effects of Point Defects and Disorder on the Vibrations of Crystals," Solid Stat. Phys. 18, 273 (1966).

[139] G. Sallen, A. Tribu, T. Aichele, R. André, L. Besombes, C. Bougerol, M. Richard, S. Tatarenko, K. Kheng, and J. P. Poizat, "Subnanosecond spectral diffusion measurement using photon correlation," Nat. Photonics 4, 696 (2010).

[140] L. C. Bassett, F. J. Heremans, C. G. Yale, B. B. Buckley, and D. D. Awschalom, "Electrical Tuning of Single Nitrogen-Vacancy Center Optical Transitions Enhanced by Photoinduced Fields," Phys. Rev. Lett. 107, 266403 (2011). 
[141] J. Walker, "Optical absorption and luminescence in diamond," Rep. Prog. Phys. 42, 1605 (1979).

[142] J. Rosa, M. Vaněček, M. Nesládek, and L. Stals, "Photoionization cross-section of dominant defects in CVD diamond," Diamond Relat. Mater. 8, 721 (1999).

[143] J. Wolters, N. Sadzak, A. W. Schell, T. Schröder, and O. Benson, "Measurement of the Ultrafast Spectral Diffusion of the Optical Transition of Nitrogen Vacancy Centers in Nano-Size Diamond Using Correlation Interferometry," Phys. Rev. Lett. 110, 027401 (2013).

[144] Y. Shen, T. M. Sweeney, and H. Wang, "Zero-phonon linewidth of single nitrogen vacancy centers in diamond nanocrystals," Phys. Rev. B 77, 033201 (2008).

[145] C. Santori, P. E. Barclay, K.-M. C. Fu, R. G. Beausoleil, S. Spillane, and M. Fisch, "Nanophotonics for quantum optics using nitrogen-vacancy centers in diamond," Nanotechnology 21, 274008 (2010).

[146] L. Robledo, H. Bernien, I. van Weperen, and R. Hanson, "Control and Coherence of the Optical Transition of Single Nitrogen Vacancy Centers in Diamond," Phys. Rev. Lett. 105, 177403 (2010).

[147] K. Beha, A. Batalov, N. B. Manson, R. Bratschitsch, and A. Leitenstorfer, "Optimum Photoluminescence Excitation and Recharging Cycle of Single Nitrogen-Vacancy Centers in Ultrapure Diamond," Phys. Rev. Lett. 109, 097404 (2012).

[148] N. Aslam, G. Waldherr, P. Neumann, F. Jelezko, and J. Wrachtrup, "Photoinduced ionization dynamics of the nitrogen vacancy defect in diamond investigated by single-shot charge state detection," New J. Phys. 15, 013064 (2013).

[149] P. Siyushev, H. Pinto, M. Vörös, A. Gali, F. Jelezko, and J. Wrachtrup, "Optically Controlled Switching of the Charge State of a Single Nitrogen-Vacancy Center in Diamond at Cryogenic Temperatures," Phys. Rev. Lett. 110, 167402 (2013).

[150] K.-M. C. Fu, C. Santori, P. E. Barclay, and R. G. Beausoleil, "Conversion of neutral nitrogen-vacancy centers to negatively charged nitrogen-vacancy centers through selective oxidation," Appl. Phys. Lett. 96, 121907 (2010).

[151] V. M. Acosta, C. Santori, A. Faraon, Z. Huang, K.-M. C. Fu, A. Stacey, D. A. Simpson, K. Ganesan, S. Tomljenovic-Hanic, A. D. Greentree, S. Prawer, and R. G. Beausoleil, "Dynamic Stabilization of the Optical Resonances of Single Nitrogen-Vacancy Centers in Diamond," Phys. Rev. Lett. 108, 206401 (2012).

[152] A. T. Collins, "The Fermi level in diamond," J. Phys. 14, 3743 (2002). 
[153] F. Treussart, V. Jacques, E. Wu, T. Gacoin, P. Grangier, and J.-F. Roch, "Photoluminescence of single colour defects in $50 \mathrm{~nm}$ diamond nanocrystals," Physica B 376, 926 (2006).

[154] L. Rondin, G. Dantelle, A. Slablab, F. Grosshans, F. Treussart, P. Bergonzo, S. Perruchas, T. Gacoin, M. Chaigneau, H.-C. Chang, V. Jacques, and J.-F. Roch, "Surface-induced charge state conversion of nitrogen-vacancy defects in nanodiamonds," Phys. Rev. B 82, 115449 (2010).

[155] M. V. Hauf, B. Grotz, B. Naydenov, M. Dankerl, S. Pezzagna, J. Meijer, F. Jelezko, J. Wrachtrup, M. Stutzmann, F. Reinhard, and J. A. Garrido, "Chemical control of the charge state of nitrogen-vacancy centers in diamond," Phys. Rev. B 83, 081304 (2011).

[156] B. Grotz, M. V. Hauf, M. Dankerl, B. Naydenov, S. Pezzagna, J. Meijer, F. Jelezko, J. Wrachtrup, M. Stutzmann, F. Reinhard, and J. A. Garrido, "Charge state manipulation of qubits in diamond," Nat. Commun. 3, 729 (2012).

[157] Y. Dumeige, F. Treussart, R. Alléaume, T. Gacoin, J. Roch, and P. Grangier, "Photo-induced creation of nitrogen-related color centers in diamond nanocrystals under femtosecond illumination," J. Lumin. 109, 61 (2004).

[158] K. Iakoubovskii, G. Adriaenssens, and M. Nesladek, "Photochromism of vacancyrelated centres in diamond," J. Phys. 12, 189 (2000).

[159] N. Manson and J. Harrison, "Photo-ionization of the nitrogen-vacancy center in diamond," Diamond Relat. Mater. 14, 1705 (2005).

[160] G. Waldherr, J. Beck, M. Steiner, P. Neumann, A. Gali, T. Frauenheim, F. Jelezko, and J. Wrachtrup, "Dark States of Single Nitrogen-Vacancy Centers in Diamond Unraveled by Single Shot NMR," Phys. Rev. Lett. 106, 157601 (2011).

[161] B. E. A. Saleh and M. C. Teich, Fundamentals of Photonics (John Wiley \& Sons, Inc., Hoboken, New Jersey, 2007), 2nd ed.

[162] C. Hood, H. Kimble, and J. Ye, "Characterization of high-finesse mirrors: Loss, phase shifts, and mode structure in an optical cavity," Phys. Rev. A 64, 033804 (2001).

[163] C. M. d. S. B. Russo, "Photon statistics of a single ion coupled to a high-finesse cavity," Ph.D. thesis, Leopold-Franzens-Universität Innsbruck (2008).

[164] E. Jaynes and F. W. Cummings, "Comparison of quantum and semiclassical radiation theories with application to the beam maser," Proc. IEEE 51, 89 (1963).

[165] Q. Turchette, R. Thompson, and H. Kimble, "One-dimensional atoms," Appl. Phys. B 60, S1 (1995). 
[166] H. J. Kimble, "Strong Interactions of Single Atoms and Photons in Cavity QED," Phys. Scr. T76, 127 (1998).

[167] F. Bernardot, P. Nussenzveig, M. Brune, J. M. Raimond, and S. Haroche, "Vacuum Rabi Splitting Observed on a Microscopic Atomic Sample in a Microwave Cavity," Europhys. Lett. 17, 33 (1992).

[168] R. J. Thompson, G. Rempe, and H. J. Kimble, "Observation of normal-mode splitting for an atom in an optical cavity," Phys. Rev. Lett. 68, 1132 (1992).

[169] G. Rempe, "Atoms in an optical cavity: quantum electrodynamics in confined space," Cont. Phys. 34, 119 (1993).

[170] M. Fox, Quantum Optics - An Introduction (Oxford University Press Inc., New York, 2006).

[171] A. Auffèves, B. Besga, J.-M. Gérard, and J.-P. Poizat, "Spontaneous emission spectrum of a two-level atom in a very-high-Q cavity," Phys. Rev. A 77, 063833 (2008).

[172] A. Auffèves, J.-M. Gérard, and J.-P. Poizat, "Pure emitter dephasing: A resource for advanced solid-state single-photon sources," Phys. Rev. A 79, 053838 (2009).

[173] A. Auffèves, D. Gerace, J.-M. Gérard, M. F. Santos, L. C. Andreani, and J.P. Poizat, "Controlling the dynamics of a coupled atom-cavity system by pure dephasing," Phys. Rev. B 81, 245419 (2010).

[174] R. Albrecht, A. Bommer, C. Deutsch, J. Reichel, and C. Becher, "Coupling of a Single Nitrogen-Vacancy Center in Diamond to a Fiber-Based Microcavity," Phys. Rev. Lett. 110, 243602 (2013).

[175] I. Wilson-Rae and A. Imamoğlu, "Quantum dot cavity-QED in the presence of strong electron-phonon interactions," Phys. Rev. B 65, 235311 (2002).

[176] G. Cui and M. G. Raymer, "Emission spectra and quantum efficiency of singlephoton sources in the cavity-QED strong-coupling regime," Phys. Rev. A 73, 053807 (2006).

[177] A. Naesby, T. Suhr, P. T. Kristensen, and J. Mørk, "Influence of pure dephasing on emission spectra from single photon sources," Phys. Rev. A 78, 045802 (2008).

[178] M. Yamaguchi, T. Asano, and S. Noda, "Photon emission by nanocavity-enhanced quantum anti-Zeno effect in solid-state cavity quantum-electrodynamics," Opt. Express 16, 18067 (2008).

[179] J. P. Reithmaier, G. Sek, A. Löffler, C. Hofmann, S. Kuhn, S. Reitzenstein, L. V. Keldysh, V. D. Kulakovskii, T. L. Reinecke, and A. Forchel, "Strong coupling in a single quantum dot-semiconductor microcavity system," Nature 432, 197 (2004). 
[180] T. Yoshie, A. Scherer, J. Hendrickson, G. Khitrova, H. Gibbs, G. Rupper, C. Ell, O. Shchekin, and D. Deppe, "Vacuum Rabi splitting with a single quantum dot in a photonic crystal nanocavity," Nature 432, 200 (2004).

[181] E. Peter, P. Senellart, D. Martrou, A. Lemaître, J. Hours, J. Gérard, and J. Bloch, "Exciton-photon strong-coupling regime for a single quantum dot embedded in a microcavity," Phys. Rev. Lett. 95, 067401 (2005).

[182] K. Hennessy, A. Badolato, M. Winger, D. Gerace, M. Atatüre, S. Gulde, S. Faelt, E. L. Hu, and A. Imamoğlu, "Quantum nature of a strongly coupled single quantum dot-cavity system," Nature 445, 896 (2007).

[183] D. Press, S. Götzinger, S. Reitzenstein, C. Hofmann, A. Löffler, M. Kamp, A. Forchel, and Y. Yamamoto, "Photon Antibunching from a Single QuantumDot-Microcavity System in the Strong Coupling Regime," Phys. Rev. Lett. 98, 117402 (2007).

[184] J. Suffczyński, A. Dousse, K. Gauthron, A. Lemaître, I. Sagnes, L. Lanco, J. Bloch, P. Voisin, and P. Senellart, "Origin of the Optical Emission within the Cavity Mode of Coupled Quantum Dot-Cavity Systems," Phys. Rev. Lett. 103, 027401 (2009).

[185] S. Ates, S. M. Ulrich, A. Ulhaq, S. Reitzenstein, A. Löffler, S. Höfling, A. Forchel, and P. Michler, "Non-resonant dot-cavity coupling and its potential for resonant single-quantum-dot spectroscopy," Nat. Photonics 3, 724 (2009).

[186] U. Hohenester, A. Laucht, M. Kaniber, N. Hauke, A. Neumann, A. Mohtashami, M. Seliger, M. Bichler, and J. J. Finley, "Phonon-assisted transitions from quantum dot excitons to cavity photons," Phys. Rev. B 80, 201311 (2009).

[187] A. Muller, E. B. Flagg, M. Metcalfe, J. Lawall, and G. S. Solomon, "Coupling an epitaxial quantum dot to a fiber-based external-mirror microcavity," Appl. Phys. Lett. 95, 173101 (2009).

[188] J. Miguel-Sánchez, A. Reinhard, E. Togan, T. Volz, A. Imamoglu, B. Besga, J. Reichel, and J. Estève, "Cavity quantum electrodynamics with charge-controlled quantum dots coupled to a fiber Fabry-Perot cavity," New J. Phys. 15, 045002 (2013).

[189] S. M. Tan, "A computational toolbox for quantum and atomic optics," J. Opt. B: Quantum Semiclassical Opt. 1, 424 (1999).

[190] W. Lukosz and R. E. Kunz, "Light emission by magnetic and electric dipoles close to a plane interface. I. Total radiated power," J. Opt. Soc. Am. 67, 1607 (1977).

[191] W. Lukosz and R. E. Kunz, "Light emission by magnetic and electric dipoles close to a plane dielectric interface. II. Radiation patterns of perpendicular oriented dipoles," J. Opt. Soc. Am. 67, 1615 (1977). 
[192] W. Lukosz, "Light emission by magnetic and electric dipoles close to a plane dielectric interface. III. Radiation patterns of dipoles with arbitrary orientation," J. Opt. Soc. Am. 69, 1495 (1979).

[193] R. R. Chance, A. Prock, and R. Silbey, "Molecular Fluorescence and Energy Transfer Near Interfaces," Adv. Chem. Phys. p. 1 (1978).

[194] A. W. Schell, G. Kewes, T. Hanke, A. Leitenstorfer, R. Bratschitsch, O. Benson, and T. Aichele, "Single defect centers in diamond nanocrystals as quantum probes for plasmonic nanostructures," Opt. Express 19, 7914 (2011).

[195] E. Neu, M. Agio, and C. Becher, "Photophysics of single silicon vacancy centers in diamond: implications for single photon emission," Opt. Express 20, 19956 (2012).

[196] K. G. Lee, X. W. Chen, H. Eghlidi, P. Kukura, R. Lettow, A. Renn, V. Sandoghdar, and S. Götzinger, "A planar dielectric antenna for directional single-photon emission and near-unity collection efficiency," Nat. Photonics 5, 166 (2011).

[197] T. Schröder, F. Gädeke, M. J. Banholzer, and O. Benson, "Ultrabright and efficient single-photon generation based on nitrogen-vacancy centres in nanodiamonds on a solid immersion lens," New J. Phys. 13, 055017 (2011).

[198] L. Marseglia, J. P. Hadden, A. C. Stanley-Clarke, J. P. Harrison, B. Patton, Y.L. D. Ho, B. Naydenov, F. Jelezko, J. Meijer, P. R. Dolan, J. M. Smith, J. G. Rarity, and J. L. O'Brien, "Nanofabricated solid immersion lenses registered to single emitters in diamond," Appl. Phys. Lett. 98, 133107 (2011).

[199] T. M. Babinec, B. J. M. Hausmann, M. Khan, Y. Zhang, J. R. Maze, P. R. Hemmer, and M. Loncar, "A diamond nanowire single-photon source," Nat. Nanotechnol. 5, 195 (2010).

[200] C. Bradac, T. Gaebel, C. I. Pakes, J. M. Say, A. V. Zvyagin, and J. R. Rabeau, "Effect of the Nanodiamond Host on a Nitrogen-Vacancy Color-Centre Emission State," Small 9, 132 (2013).

[201] A. Mohtashami and A. F. Koenderink, "Suitability of nanodiamond nitrogen vacancy centers for spontaneous emission control experiments," New J. Phys. 15, 043017 (2013).

[202] Y. Liang, M. Ozawa, and A. Krueger, "A General Procedure to Functionalize Agglomerating Nanoparticles Demonstrated on Nanodiamond," ACS Nano 3, 2288 (2009).

[203] A. Krueger and D. Lang, "Functionality is Key: Recent Progress in the Surface Modification of Nanodiamond," Adv. Funct. Mater. 22, 890 (2012). 
[204] S. Cui and E. L. Hu, "Increased negatively charged nitrogen-vacancy centers in fluorinated diamond," Appl. Phys. Lett. 103, 051603 (2013).

[205] M. Ozawa, M. Inaguma, M. Takahashi, F. Kataoka, A. Krüger, and E. Ōsawa, "Preparation and Behavior of Brownish, Clear Nanodiamond Colloids," Adv. Mater. 19, 1201 (2007).

[206] E. Neu, F. Guldner, C. Arend, Y. Liang, S. Ghodbane, H. Sternschulte, D. Steinmüller-Nethl, A. Krueger, and C. Becher, "Low temperature investigations and surface treatments of colloidal narrowband fluorescent nanodiamonds," J. Appl. Phys. 113, 203507 (2013).

[207] J. Tisler, G. Balasubramanian, B. Naydenov, R. Kolesov, B. Grotz, R. Reuter, J.-P. Boudou, P. A. Curmi, M. Sennour, A. Thorel, M. Börsch, K. Aulenbacher, R. Erdmann, P. R. Hemmer, F. Jelezko, and J. Wrachtrup, "Fluorescence and Spin Properties of Defects in Single Digit Nanodiamonds," ACS Nano 3, 1959 (2009).

[208] M. E. Trusheim, L. Li, A. Laraoui, E. H. Chen, H. Bakhru, T. Schröder, O. Gaathon, C. A. Meriles, and D. Englund, "Scalable Fabrication of High Purity Diamond Nanocrystals with Long-Spin-Coherence Nitrogen Vacancy Centers," Nano Lett. 14, 32 (2014).

[209] A. Krüger, Neue Kohlenstoffmaterialien (B.G. Teubner Verlag, Wiesbaden, 2007), 1 st ed.

[210] W.-H. Wang and S. Chao, "Annealing effect on ion-beam-sputtered titanium dioxide film," Opt. Lett. 23, 1417 (1998).

[211] Y. Zhao, Y. Wang, H. Gong, J. Shao, and Z. Fan, "Annealing effects on structure and laser-induced damage threshold of Ta2O5/SiO2 dielectric mirrors," Appl. Surf. Sci. 210, 353 (2003).

[212] D. Steinmetz, "Ni/Si-basierte Farbzentren in Diamant als Einzelphotonenquellen," Ph.D. thesis, Universität des Saarlandes (2011).

[213] R. Long, T. Steinmetz, P. Hommelhoff, W. Hänsel, T. W. Hänsch, and J. Reichel, "Magnetic microchip traps and single-atom detection," Philos. Trans. R. Soc. London, Ser. A 361, 1375 (2003).

[214] Y. Colombe, T. Steinmetz, G. Dubois, F. Linke, D. Hunger, and J. Reichel, "Strong atom-field coupling for Bose-Einstein condensates in an optical cavity on a chip," Nature 450, 272 (2007).

[215] M. Steiner, H. M. Meyer, C. Deutsch, J. Reichel, and M. Köhl, "Single Ion Coupled to an Optical Fiber Cavity," Phys. Rev. Lett. 110, 043003 (2013). 
[216] A. Muller, E. B. Flagg, J. R. Lawall, and G. S. Solomon, "Ultrahigh-finesse, low-mode-volume Fabry-Perot microcavity," Opt. Lett. 35, 2293 (2010).

[217] C. Toninelli, Y. Delley, T. Stöferle, A. Renn, S. Götzinger, and V. Sandoghdar, "A scanning microcavity for in situ control of single-molecule emission," Appl. Phys. Lett. 97, 021107 (2010).

[218] N. E. Flowers-Jacobs, S. W. Hoch, J. C. Sankey, A. Kashkanova, A. M. Jayich, C. Deutsch, J. Reichel, and J. G. E. Harris, "Fiber-cavity-based optomechanical device," Appl. Phys. Lett. 101, 221109 (2012).

[219] L. Collot, V. Lefèvre-Seguin, M. Brune, J. M. Raimond, and S. Haroche, "Very High- Q Whispering-Gallery Mode Resonances Observed on Fused Silica Microspheres," Europhys. Lett. 23, 327 (1993).

[220] D. K. Armani, T. J. Kippenberg, S. M. Spillane, and K. J. Vahala, "Ultra-high-Q toroid microcavity on a chip," Nature 421, 925 (2003).

[221] D. Hunger, "Herstellung und Charakterisierung von Faserresonatoren hoher Finesse," Master's thesis, Ludwig-Maximilians-Universität München (2005).

[222] C. Deutsch, "High Finesse fibre Fabry-Perot resonators - Production, characterization and applications," Master's thesis, Ludwig-Maximilians Universität München (2008).

[223] D. Hunger, C. Deutsch, R. J. Barbour, R. J. Warburton, and J. Reichel, "Laser micro-fabrication of concave, low-roughness features in silica," AIP Adv. 2, 012119 (2012).

[224] B. Petrak, K. Konthasinghe, S. Perez, and A. Muller, "Feedback-controlled laser fabrication of micromirror substrates," Rev. Sci. Instrum. 82, 123112 (2011).

[225] P. R. Dolan, G. M. Hughes, F. Grazioso, B. R. Patton, and J. M. Smith, "Femtoliter tunable optical cavity arrays," Opt. Lett. 35, 3556 (2010).

[226] T. Steinmetz, Y. Colombe, D. Hunger, T. W. Hänsch, A. Balocchi, R. J. Warburton, and J. Reichel, "Stable fiber-based Fabry-Pérot cavity," Appl. Phys. Lett. 89, 111110 (2006).

[227] D. M. B. Kunert, T. P. Meyrath, and H. Giessen, "Fabrication of a fiber-based microcavity with spherical concave fiber tips," Appl. Phys. B 98, 707 (2010).

[228] M. Trupke, E. A. Hinds, S. Eriksson, E. A. Curtis, Z. Moktadir, E. Kukharenka, and M. Kraft, "Microfabricated high-finesse optical cavity with open access and small volume," Appl. Phys. Lett. 87, 211106 (2005).

[229] D. Hunger, T. Steinmetz, Y. Colombe, C. Deutsch, T. W. Hänsch, and J. Reichel, "A fiber Fabry-Perot cavity with high finesse," New J. Phys. 12, 065038 (2010). 
[230] G. E. Walrafen and J. Stone, "Raman Spectral Characterization of Pure and Doped Fused Silica Optical Fibers," Appl. Spectrosc. 29, 337 (1975).

[231] R. H. Stolen, "Nonlinearity in fiber transmission," Proc. IEEE 68, 1232 (1980).

[232] R. H. Stolen, J. P. Gordon, W. J. Tomlinson, and H. A. Haus, "Raman response function of silica-core fibers," J. Opt. Soc. Am. B 6, 1159 (1989).

[233] M. Dračínský, L. Benda, and P. Bouř, "Ab initio modeling of fused silica, crystal quartz, and water Raman spectra," Chem. Phys. Lett. 512, 54 (2011).

[234] F. Ruebel, P. Haag, and J. A. L'huillier, "Synchronously pumped femtosecond optical parametric oscillator with integrated sum frequency generation," Appl. Phys. Lett. 92, 011122 (2008).

[235] U. Fano, "Effects of Configuration Interaction on Intensities and Phase Shifts," Phys. Rev. 124, 1866 (1961).

[236] M. Galli, S. L. Portalupi, M. Belotti, L. C. Andreani, L. O'Faolain, and T. F. Krauss, "Light scattering and Fano resonances in high-Q photonic crystal nanocavities," Appl. Phys. Lett. 94, 071101 (2009).

[237] R. V. Pound, "Electronic Frequency Stabilization of Microwave Oscillators," Rev. Sci. Instrum. 17, 490 (1946).

[238] R. Drever, J. Hall, F. Kowalski, J. Hough, G. Ford, A. Munley, and H. Ward, "Laser phase and frequency stabilization using an optical resonator," Appl. Phys. B 31, 97 (1983).

[239] E. D. Black, "An introduction to Pound-Drever-Hall laser frequency stabilization," Am. J. Phys. 69, 79 (2001).

[240] A. Bommer, "Aufbau eines fasergekoppelten Mikroresonators zur Kopplung von Farbzentren in Diamant bei kryogenen Temperaturen," Master's thesis, Universität des Saarlandes (2013).

[241] E. Neu, D. Steinmetz, J. Riedrich-Moeller, S. Gsell, M. Fischer, M. Schreck, and C. Becher, "Single photon emission from silicon-vacancy colour centres in chemical vapour deposition nano-diamonds on iridium," New J. Phys. 13, 025012 (2011).

[242] Y. Dumeige, M. Chipaux, V. Jacques, F. Treussart, J.-F. Roch, T. Debuisschert, V. M. Acosta, A. Jarmola, K. Jensen, P. Kehayias, and D. Budker, "Magnetometry with nitrogen-vacancy ensembles in diamond based on infrared absorption in a doubly resonant optical cavity," Phys. Rev. B 87, 155202 (2013).

[243] E. Gross, "Spektroskopische Untersuchung von Farbzentren in Diamant im sichtbaren und infraroten Spektralbereich," Master's thesis, Univeristät des Saarlandes (2011). 
[244] E. K. Neu, "Silicon vacancy color centers in chemical vapor deposition diamond: New insights into promising solid state single photon sources," Ph.D. thesis, Universität des Saarlandes (2012). 


\section{Publications}

\section{Journal publications}

1. R. Albrecht, A. Bommer, C. Deutsch, J. Reichel, and C. Becher, "Coupling of a Single Nitrogen-Vacancy Center in Diamond to a Fiber-Based Microcavity",Phys. Rev. Lett. 110, 243602 (2013).

2. E. Neu, R. Albrecht, M. Fischer, S. Gsell, M. Schreck, and C. Becher, "Electronic transitions of single silicon vacancy centers in the near-infrared spectral region", Phys. Rev. B 85, 245207 (2012).

3. S. Zaske, A. Lenhard, C.A. Keßler, J. Kettler, C. Hepp, C. Arend, R. Albrecht, W.-M. Schulz, M. Jetter, P. Michler, and C. Becher, "Visible-to-Telecom Quantum Frequency Conversion of Light from a Single Quantum Emitter", Phys. Rev. Lett. 109, 147404 (2012).

4. D. Steinmetz, E. Neu, C. Hepp, R. Albrecht, W. Bolse, J. Meijer, D. Rogalla and C. Becher, "Single-photon emission from Ni-related color centers in CVD diamond", Proc. SPIE 7727, 77270P (2010). 


\section{Conference Papers ${ }^{1}$}

1. CLEO/Europe EQEC 2013, Munich, Germany:

-R. Albrecht, A. Bommer, C. Deutsch, J. Reichel and C. Becher, "Coupling of a single $\mathrm{N}-\mathrm{V}$ center in diamond to a fiber-based microcavity", in CLEO/Europe and EQEC 2013 Conference Digest, OSA Technical Digest (CD) (Optical Society of America, 2013), paper IH3.5

2. CLEO/Europe EQEC 2013, Munich, Germany:

-A. Lenhard, S. Zaske, C. Keßler, J. Kettler, C. Arend, C. Hepp, R. Albrecht, W.-M. Schulz, M. Jetter, and P. Michler and C. Becher, "Quantum Frequency Conversion of Visible Single Photons from a Quantum Dot to a Telecom Band", in CLEO/Europe and EQEC 2013 Conference Digest, OSA Technical Digest (CD) (Optical Society of America, 2013), paper IA3.5

3. NOEKS 2012, Stuttgart, Germany:

-C. Becher, A. Lenhard, S. Zaske, C. Keßler, J. Kettler, C. Arend, C. Hepp,

R. Albrecht, W.-M. Schulz, M. Jetter, and P. Michler, "Quantum Frequency Conversion of Single Photons from a Quantum Dot to the Telecom Band", 11th International, paper Th1.1

4. CLEO/QELS 2012, San Jose, CA, USA:

-A. Lenhard, S. Zaske, C. Keßler, J. Kettler, C. Arend, C. Hepp, R. Albrecht, W. Schulz, M. Jetter, P. Michler, and C. Becher, "Quantum Frequency DownConversion of Single Photons from a Quantum Dot to the Telecom Band", in CLEO:2012 (Optical Society of America, Washington, DC, 2012), paper QTh5B.6., Postdeadline paper

5. Hasselt Diamond Workshop 2010, Hasselt, Belgium

-E. Neu, D. Steinmetz, C. Hepp, R. Albrecht, W. Bolse, J. Meijer and C. Becher, "Characterization of single Nickel-related color centers created by ion-implantation", Hasselt Diamond Workshop 2010, SBDD XV

6. QIPC 2009, Rome, Italy:

R. Albrecht, J. Riedrich-Möller, E. Neu, D. Steinmetz, C. Hepp, B. Sauer, M. Hauschild and $\bullet \mathrm{C}$. Becher, "Towards optical interfaces for color centers in diamond", International Conference on Quantum Information Processing and Communication, QIPC 2009

- marks the presenting author 
7. CLEO/Europe EQEC 2009 Munich, Germany:

-D. Steinmetz, R. Albrecht, E. Neu, S. Ghodbane, D. Steinmüller-Nethl, S. Deutsch, J. Reichel und C. Becher, "Towards optimized single photon sources based on color centers in diamond", CLEO Europe - EQEC 2009, 10.1109, art. no. 5191456

8. CLEO/QELS and PhAST 2008, San Jose, CA, USA:

J. Riedrich-Möller, C. Kreuzer, R. Albrecht, E. Neu, and •C. Becher, "Design of Photonic Crystal Microcavities in Diamond Films for Quantum Information", in Conference on Lasers and Electro-Optics, OSA Technical Digest (Optical Society of America, 2008), paper JThA103 



\section{Danke!}

Zum Erfolg dieser Arbeit haben viele beigetragen. Ganz besonders danken möchte ich meinem Betreuer, Christoph Becher, für das Vertrauen und die Möglichkeit bei ihm zu promovieren und ein Teil der Forschung in einem sehr spannenden und hochaktuellen Gebiet zu sein. Es war eine herausfordernde und sehr lehrreiche Zeit, die mein Leben sehr bereichert hat. Vielen Dank!

I am also very grateful to Jason Smith who agreed to be one of the examiners for my thesis. I really do appreciate it and feel honored to have an international expert on the field reviewing my findings.

Ich hatte das Glück auf Kooperationspartner zurückgreifen zu können, welche diese Arbeit erst ermöglicht haben. Ein grosses Danke an Jakob Reichel und Christian Deutsch mit denen gemeinsam ich ein Grossteil der Fasern herstellen konnte. Ein Dank gebührt auch Oliver Benson und Andreas Schell für das Ablegen eines Diamanten auf einer Faser, was mir zusätzliche Experimente ermöglicht hat.

Auch hier an der Uni konnte ich auf zahlreiche Hilfe zählen. Dank geht an Christoph Pauly für die meisterhafte Bedienung des FIBs, Ludovic Marquant für die Einführung in die Spin-Coating Technik, Christian Zeitz für die AFM Messungen, Jörg Schmauch für Elektronenmikroskopaufnahmen, Marc Bienert, für die Expertise in Theorie-Fragen, Karin Kretsch für die Unterstützung im Chemielabor, Stephan Löw für die Assistance in Elektronikbelangen und dem gesamten Team der Feinmechanik-Werkstatt insbesondere Michael Schmitt und Peter Wagner für die zahlreich hergestellten Bestandteile meines Aufbaus. Ganz besonders Danke sagen möchte ich unserer Sekretärin Elke Huschens für den Überblick im chaotischen Uni-Betrieb und die riesige Hilfsbereitschaft.

Ein grosses Dankeschön auch an meine aktuellen und ehemaligen Kollegen. Ohne das Teamwork und die gegenseitige Unterstützung wäre diese Arbeit nicht möglich gewesen. Insbesondere mein dezidierter Nachfolger Alexander, den ich sowohl in der Bachelor-, als auch Masterarbeit betreuen durfte, hat viel zum Gelingen beigetragen. Durch stetes Hinterfragen wurde die Theorie und Methoden stetig geprüft und letztlich 
gefestigt. Zudem bin ich Alexander auch sehr dankbar für das Nachrechnen der Theorie und das Korrekturlesen dieser Arbeit. Christian bin ich für die Hilfe bei Problemen des Konfokalsetups sehr zu Dank verpflichtet. Auch die Automatisierung dieses Setups hat viel Arbeit enorm erleichtert. Janine vielen Dank für die Unterstützung bei den ganzen Simulationen. Sebastians viele auflockernde Sprüche, die lehrreichen Beispiele im Umgang mit der Verwaltung, die Tipps für die Bewerbungen und die Layoutvorlage für die Dissertation haben mir sehr geholfen. Ich hatte das Glück über Jahre mit Elke das Büro zu teilen und viel von ihrem riesigen Wissen und unglaublichen Gedächtnis, in welchem Paper was steht, zu profitieren. Andreas Fuchs danke ich für die stets gute Laune und den Spincoater, welcher mir hervorragende Dienste erwiesen hat. David und Carsten, ein grosses Dankeschön für das Kümmern um den ganzen Informatik-Kram und die entsprechende Unterstützung. Für die Einführung in die pfälzische Kultur, insbesondere der Weinfeste und für die zahlreichen Paper, bei denen ich auf seinen Zugriff zählen konnte möchte ich Andreas Lenhard danken. Ein Dank natürlich auch an Benjamin, welcher mit seiner Diplomarbeit unter meiner Betreuung die Arbeit um einiges weiter gebracht hat. Weiter hatte ich auch das Vergnügen Matthias als Bachelorstudenten zu haben.

Laura und Sarah euch ganz riesigen Dank für die zahlreichen gemeinsamen Aktivitäten wie Spieleabende, Kochen oder Klettern, die über den normalen Uni-Alltag hinaus gingen und für die notwendige Abwechslung gesorgt haben. Insbesondere hast du Laura mich durch Bekanntmachung mit Frisbee in einer eher zähen Phase dieser Arbeit den nötigen Elan gegeben, um die zweite Halbzeit erfolgreich in Angriff zu nehmen. Die Freundschaft, die daraus entstanden ist, schätze ich sehr. Auch allen weiteren Teammitglieder von OOUPS, den Original Organic Ultimate Players Saarbrücken, vielen Dank. Ohne Ultimate hätte ich es so lange nicht in Saarbrücken ausgehalten. Besonderen Dank gebührt Jörg, dessen Begeisterungsfähigkeit mich unter anderem auch zum erfolgreichen Absolvieren eines Marathons gebracht hat. Nicht vergessen will ich meine Freunde der ersten Stunde in Saarbrücken, Patricia und Elisa. Die vielen schönen gemeinsamen Momente haben dafür gesorgt, dass ich mich in Saarbrücken stets wohl gefühlt habe. Ein Dank geht auch an Christoph Mudrich und Windmachine. Die schöne Zeit, tolle Konzerte und insbesondere die Berlin-Tour werden mir stets in guter Erinnerung bleiben. 
Ganz besonders dankbar bin ich meiner Familie, die mich auf meinem ganzen bisherigen Weg immer unterstützt hat. Vielen Dank für den stets vorhandenen Rückhalt, die Geduld und viel Verständnis. Ich schätze mich unglaublich glücklich euch zu haben.

Enormes weiteres Glück hast auch du Sarah, mein Schatz, mir in der Schlussphase dieser Dissertation bereitet und mir den Weg in eine mir zuvor unbekannte Zukunft geöffnet. Ich bin ganz froh, nicht der schnellste gewesen zu sein und dich so kennengelernt zu haben. 